\title{
MANAGING EMPLOYEE PERFORMANCE IN TRANSITION ECONOMIES: A STUDY OF VIETNAMESE PUBLIC ORGANISATIONS
}

\author{
By \\ VU ANH TAI
}

\begin{abstract}
A thesis
submitted to the Victoria University of Wellington in fulfilment of the requirements for the degree of Doctor of Philosophy
\end{abstract}

Victoria University of Wellington

2019 


\begin{abstract}
Public employees' poor performance has been cited as one of the leading factors in the failure of reform programmes in developing countries. Although previous employee performance reforms have targeted selection, training, appraisal and compensation, agreement exists that these problems persist. Improving employee performance has proven difficult in developing and developed countries alike.

Motivated by the New Public Management (NPM), employee performance management (PM) has been widely introduced by developed and developing countries as a public sector reform. Employee PM supposedly furthers development objectives by improving performance, enhancing accountability and aligning employee efforts with organisational goals. Unfortunately, the literature has reported many failed PM reforms. Arguably, employee PM in the public sector usually faces several difficulties, including the diversity of stakeholders, bounded delegated authority, ambiguous organisational and job goals, as well as the inherent complexity of employee PM activities. The situation is even more challenging for developing countries due to contextual problems of weak institutions, weak capacity and traditional cultures. There are perspectives that employee PM may not be compatible in developing contexts.
\end{abstract}

Although there is no shortage of research on employee PM, most is theoretical research or conducted in developed countries. Empirical research in developing countries, particularly in the public sector, remains sparse. To address this gap, this research investigates whether employee PM is applicable or effective in the developing context, as well as exploring which contextual factors affect its development.

To answer these research questions, a mixed methods approach guided by the research philosophy of pragmatism was adopted. The data for this research was gathered from 30 interviews and a survey of 322 respondents from 29 different organisations across five central Ministries and two provinces in Vietnam as a transitional economy with a strong effect of Confucian culture.

This study contributes to the literature by providing some key findings. Firstly, it confirms that if well designed and implemented, PM can work in the public sector in developing countries. This finding supports the perspective that the failure of PM schemes is mostly because of implementation shortcomings rather than theory defects. Secondly, it 
proposes a formula for the effective implementation of PM in the developing context. Specifically, it is a combination of five PM practices, including goal-based appraisal, feedback, reward-for-performance, addressing poor performers and employee participation. Thirdly, the development of employee PM in developing countries is driven by three contextual factors: agency accountability, HR autonomy, and entrepreneurial leadership. Fourthly, PM is not only a tool to improve organisational performance, but also an important mediation agent to transmit the effect of other reform activities on desirable outcomes. Finally, the effect of the contextual factors on development of employee PM is weakened by interpersonal relationships and nepotism while being strengthened by communication and training.

Based on these findings, this research proposes strategic solutions for policy-makers while providing specific suggestions for practitioners to develop effective PM systems. It also discusses some implications and identifies gaps for researchers in the future. 


\section{Acknowledgements}

Firstly, I would like to express my deepest gratitude to my two respected supervisors, Dr. Geoff Plimmer and Professor Evan Berman for their excellent supervision and tremendous assistance. Without their tireless guidance, I am not sure that I would have come to the end of this very challenging journey.

My sincere gratitude also goes to Dr. Richard Norman, my former primary supervisor, who supported me a lot in the initial period of the study. His advice and mentoring enabled me to acquire background knowledge that has been so significant for my subsequent study.

I am very grateful to the New Zealand Government's International Aid and Development Programme that brought me to New Zealand, a beautiful country, to pursue this interesting but challenging research. Without this generous assistance, I could not reach today's achievement.

I would surely be remiss if I did not acknowledge the staff and $\mathrm{PhD}$ programme directors in the School of Management, Victoria University of Wellington who have enthusiastically assisted me throughout the study process.

I would like to express my huge appreciation to all my research respondents for their time and valuable information. Indeed, this information gave me in-depth insights into my study issues and is an indispensable part of this thesis.

Last but by no means least, I want to express my thanks to all my family members, particularly my wife, Nga Thanh Tran and our two children Hai Anh Vu and Linh Thi My Vu. I know that they have had a tough time when I could not be at home to take care of them. Together, they have been the source of enormous encouragement so that I was able to overcome challenges in the study process.

I dedicate this thesis to my supervisors and my family with my deepest thanks. 


\section{Table of contents}

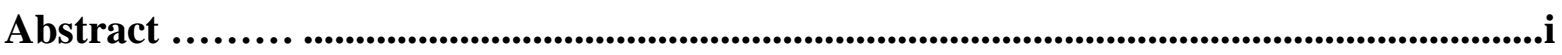

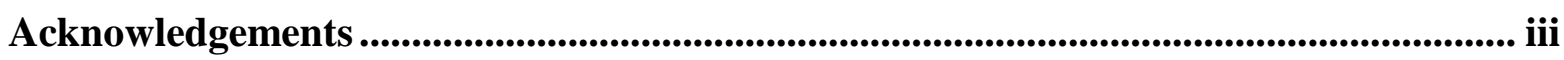

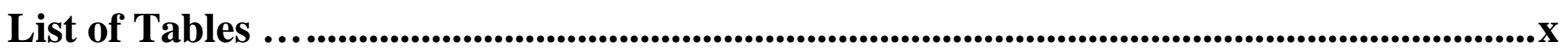

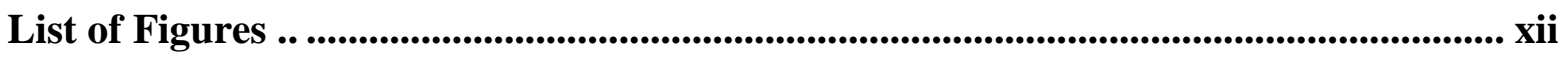

Main abbreviations and acronyms..................................................................................................... xiii

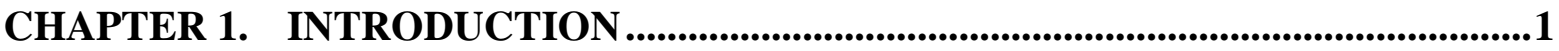

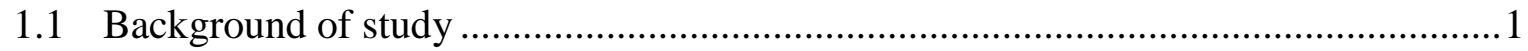

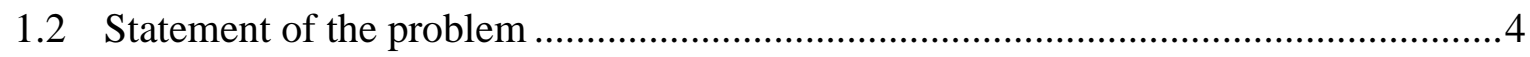

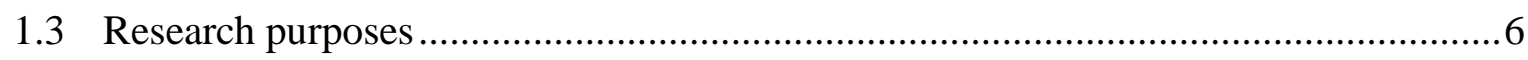

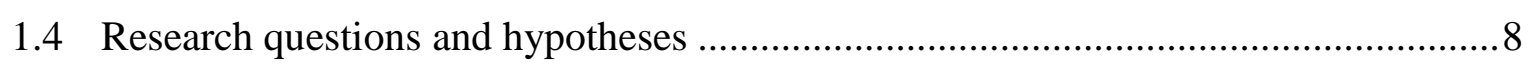

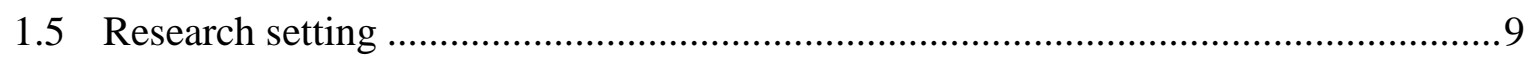

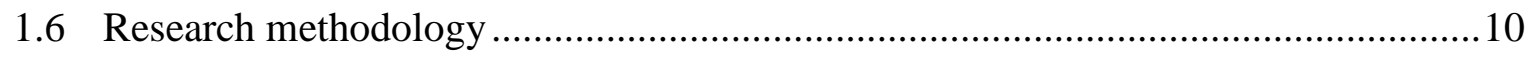

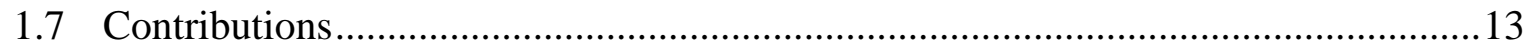

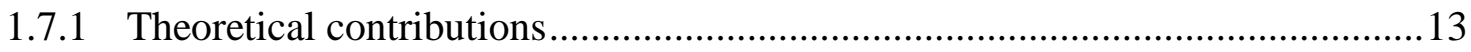

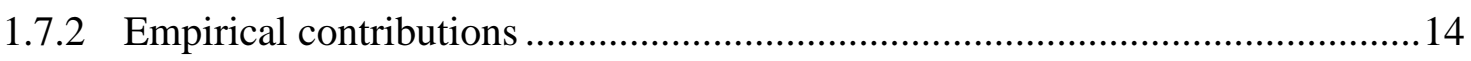

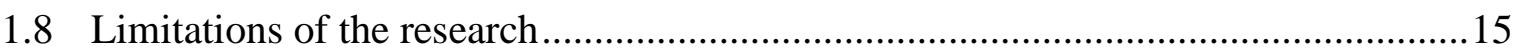

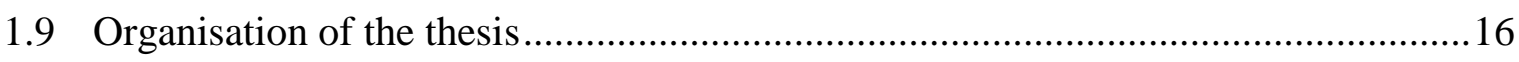

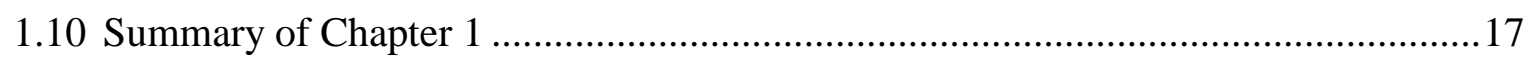

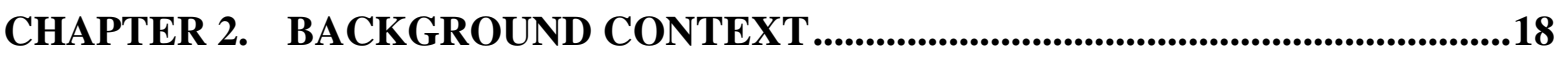

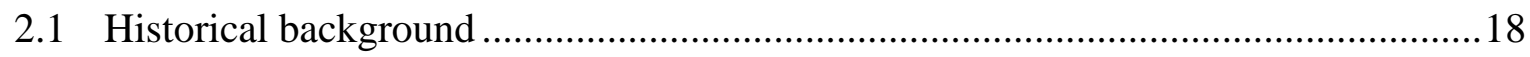

2.2 Public administration reform programmes.......................................................... 19

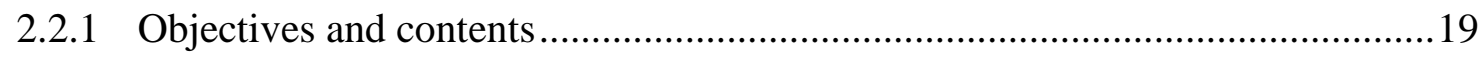

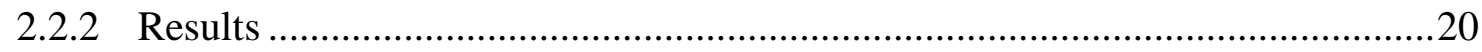

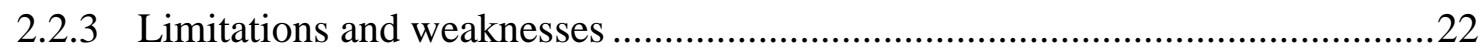

2.3 Overview of HRM policies in the Vietnam public sector......................................24

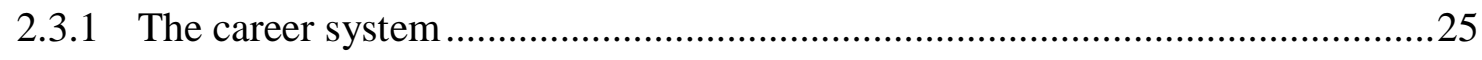

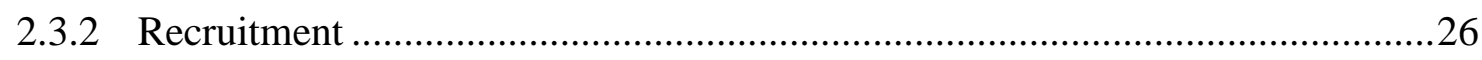

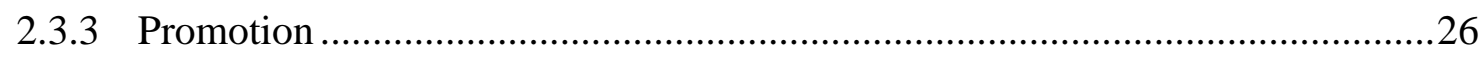

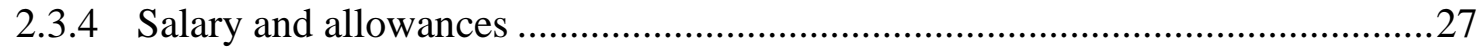




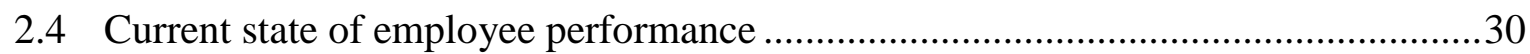

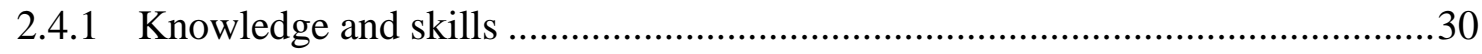

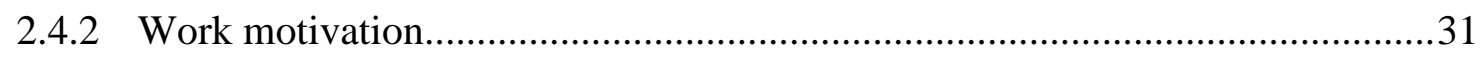

2.5 Transiting from traditional appraisal to performance management ............................32

2.5.1 The appraisal practice prior to initiating the reform policy .............................. 32

2.5.2 The appraisal practice from the beginning of the reform policy to the passage of

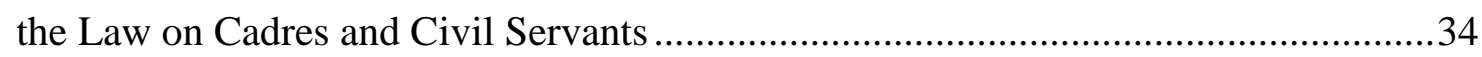

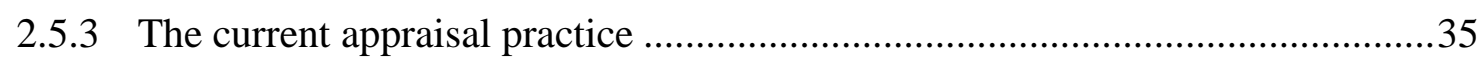

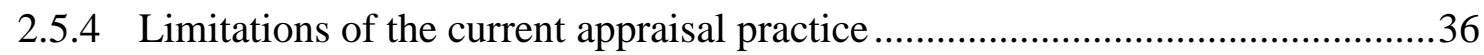

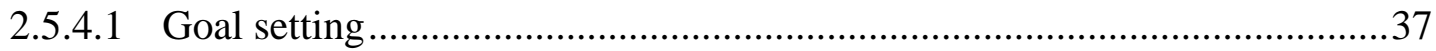

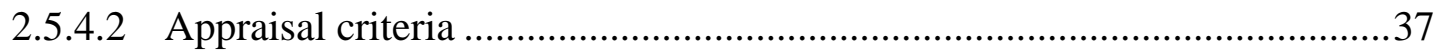

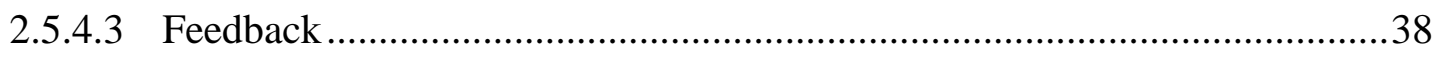

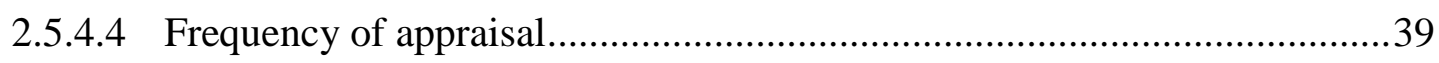

2.5.4.5 Connection to rewards and other personnel practices ...............................40

2.5.5 Innovation towards performance management ...................................... 41

2.5.5.1 Antecedents for innovation towards performance management .................41

2.5.5.2 Characteristics of the innovation of performance management practice ....43

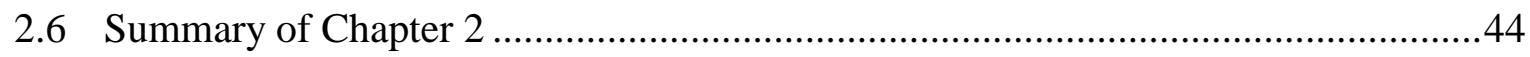

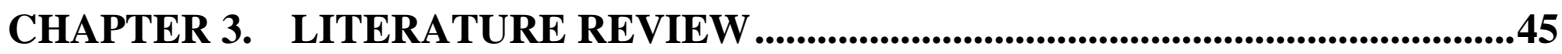

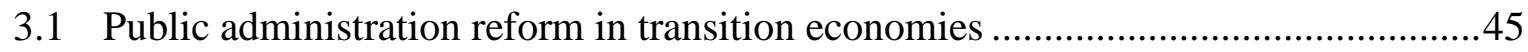

3.2 Evolution and definition of employee performance management ............................47

3.2.1 Evolution of employee performance management........................................47

3.2.2 Definition of employee performance management .....................................49

3.3 Introduction and challenges of employee performance management.......................50

3.3.1 Employee performance management in the public sector...............................50

3.3.2 Challenges of employee performance management in developing countries ....51

3.4 Employee performance management practices in transitional countries ...................53 


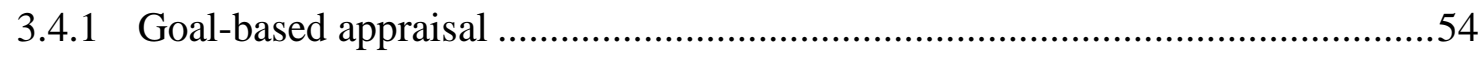

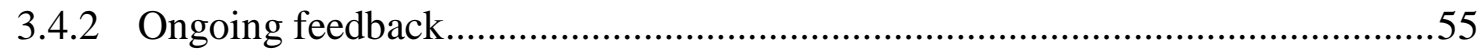

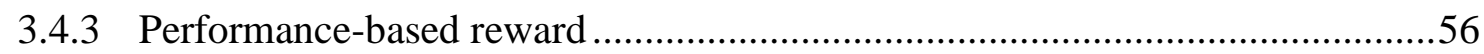

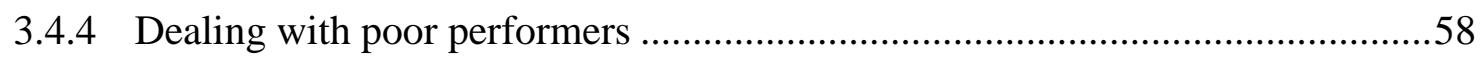

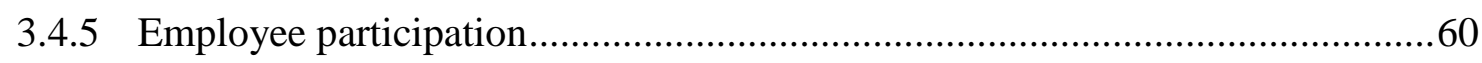

3.5 Outcomes of employee performance management and underpinning theories .........62

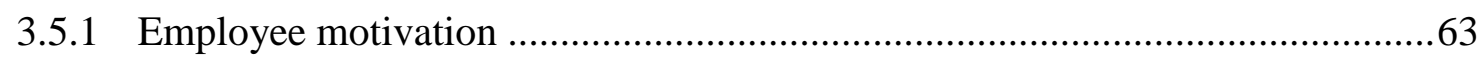

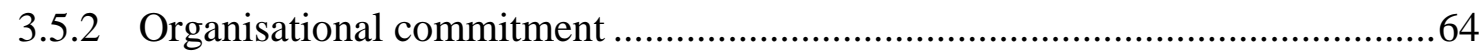

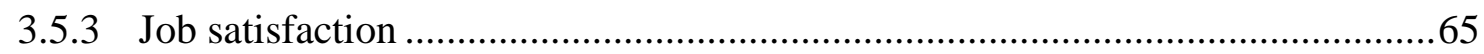

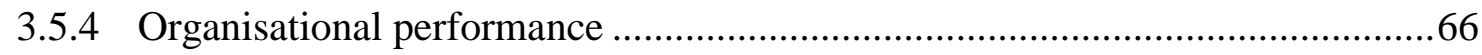

3.6 The effect of contextual factors on employee performance management ................68

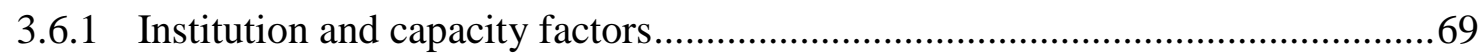

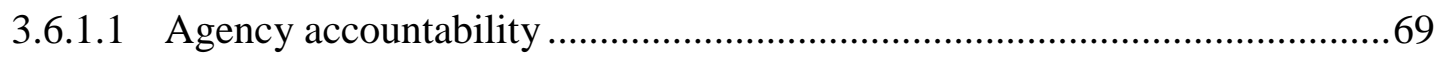

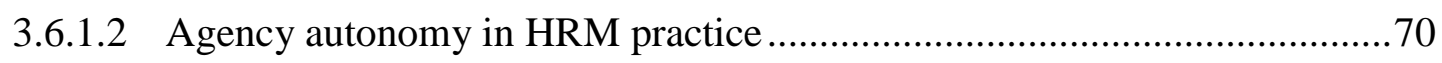

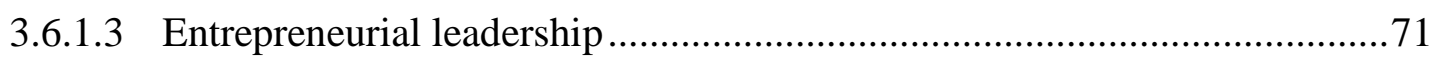

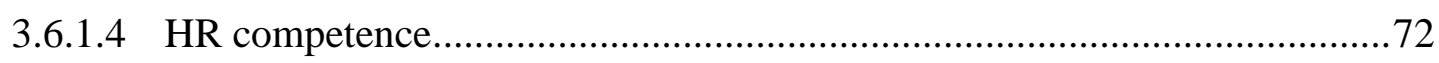

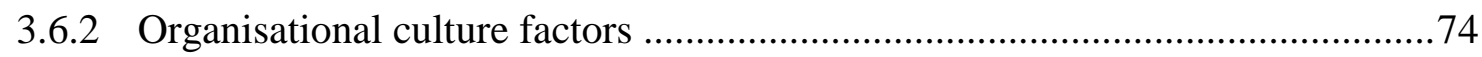

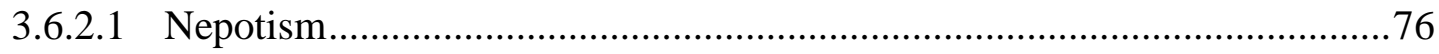

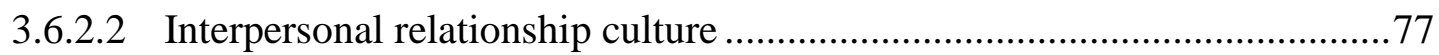

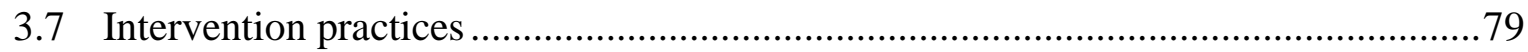

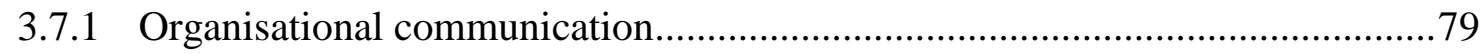

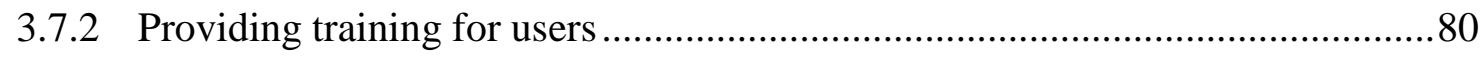

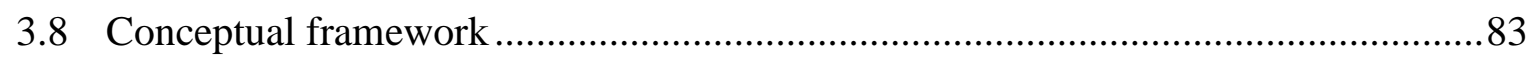

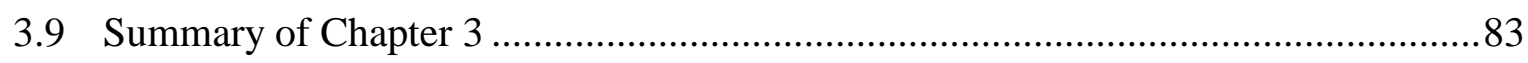

CHAPTER 4. RESEARCH METHODOLOGY ......................................................85

4.1 The selection of paradigm and research approach .............................................. 85

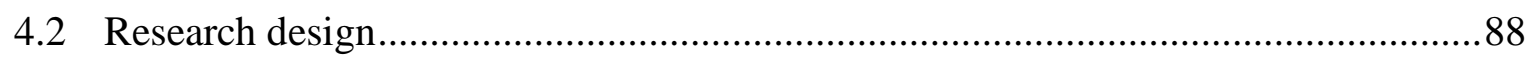

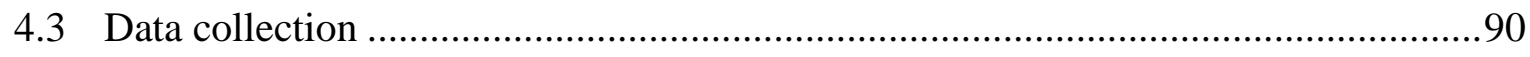

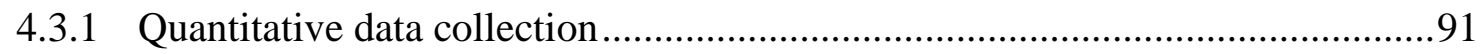


4.3.1.1 Population and sample identification ....................................................

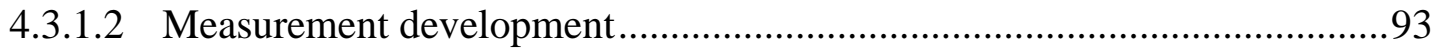

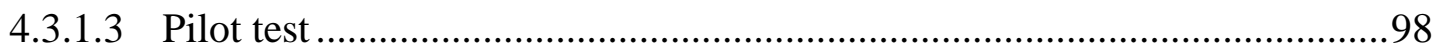

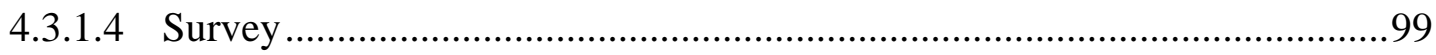

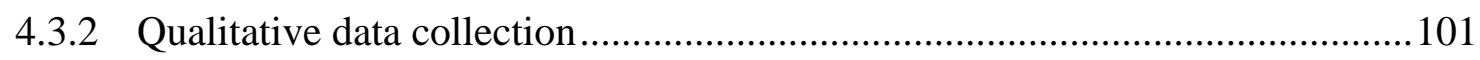

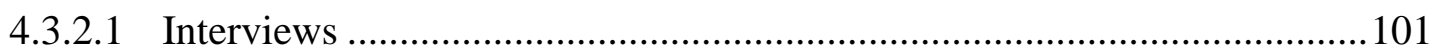

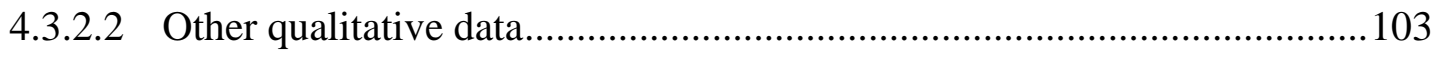

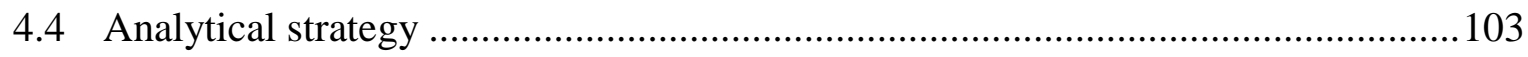

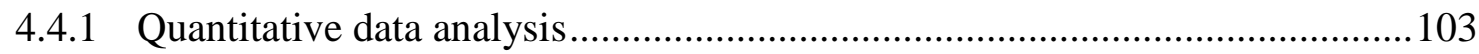

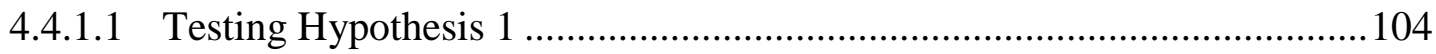

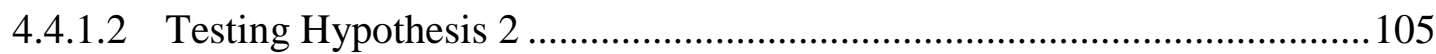

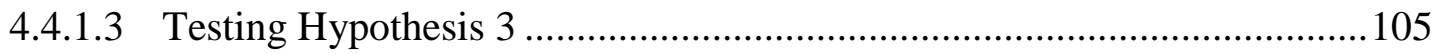

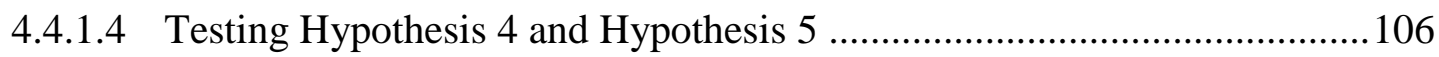

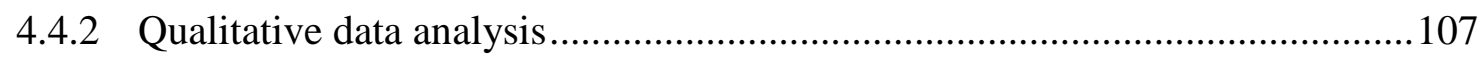

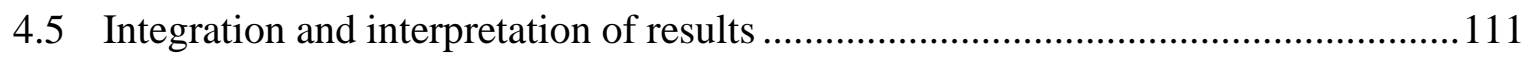

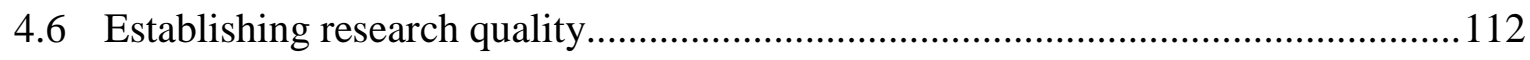

4.6.1 Establishing and testing reliability and validity in the quantitative strand.......112

4.6.1.1 Establishing and testing reliability ...................................................112

4.6.1.2 Establishing and testing validity ......................................................... 113

4.6.2 Establishing validity and reliability in the qualitative strand ........................114

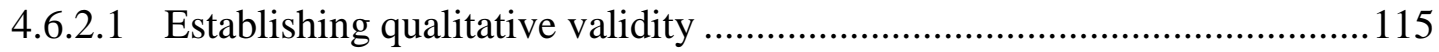

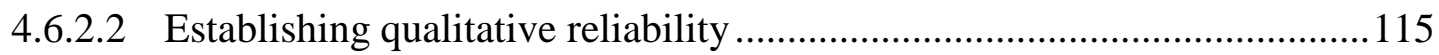

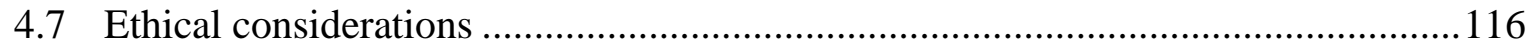

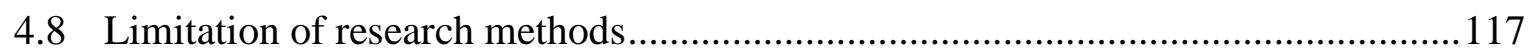

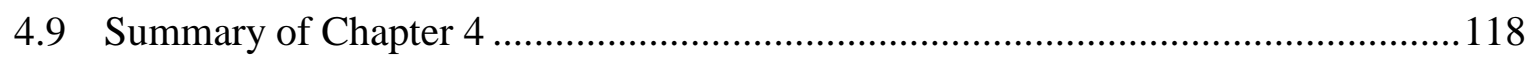

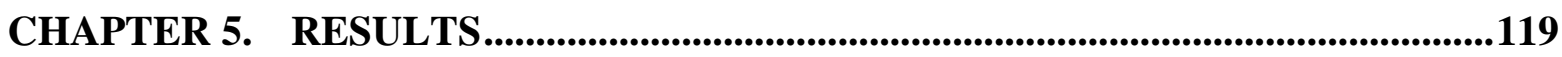

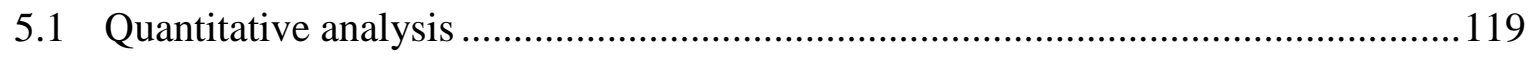

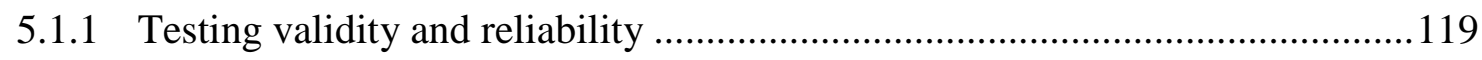


5.1.2.1 Hypothesis 1: the effect of employee PM on outcome variables

5.1.2.2 Hypothesis 2: the effect of contextual variables on employee PM

5.1.2.3 Hypothesis 3: the effect of contextual variables on the outcome variables ... 133

5.1.2.4 Hypothesis 4: the moderation role of cultural variables 136

5.1.2.5 Hypothesis 5: the moderation role of intervention variables 140

5.2 Qualitative analysis 143

5.2.1 The development of employee PM and the outcome effectiveness 144

5.2.2 How the contextual factors influence the development of employee PM

5.2.3 How the contextual variables affect the outcome variables 154

5.2.4 How the cultural factors affect employee PM 156

5.2.5 How intervention practices strengthen employee PM. 159

5.2.6 Other findings from qualitative data analysis 161

5.3 Integrating the quantitative and qualitative findings 163

5.4 Summary of Chapter 5 167

CHAPTER 6. DISCUSSION, IMPLICATIONS AND CONCLUSIONS 168

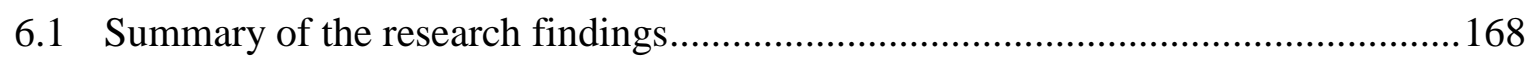

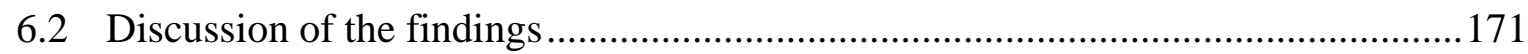

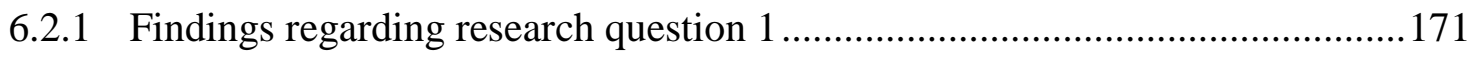

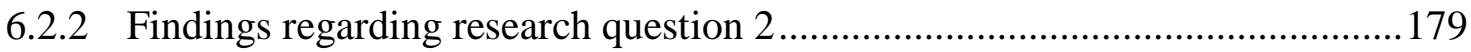

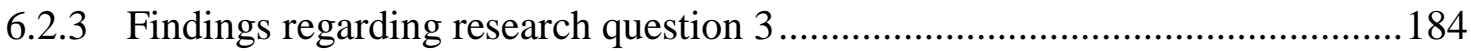

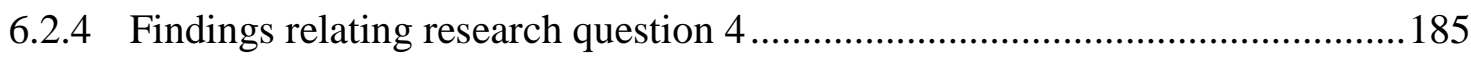

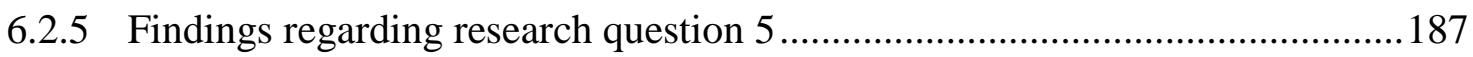

6.2.6 Other findings from the qualitative data.................................................. 190

6.2.7 Proposing a theoretical model from the findings ........................................191

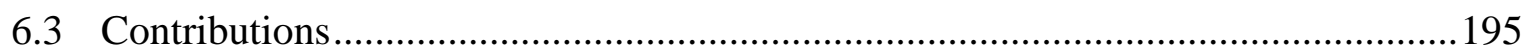

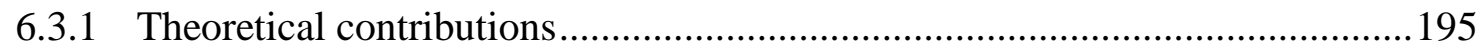

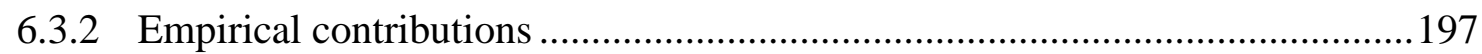

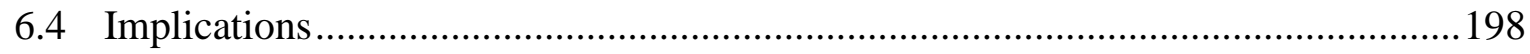

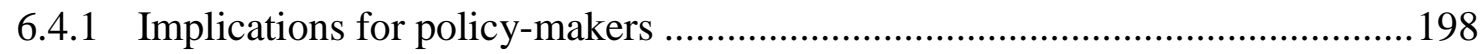




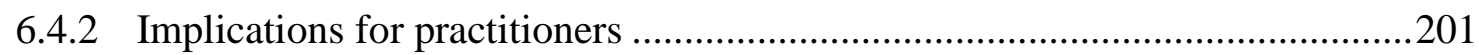

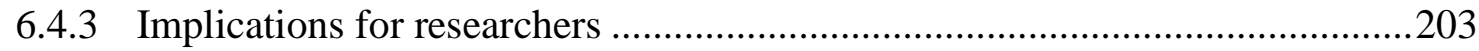

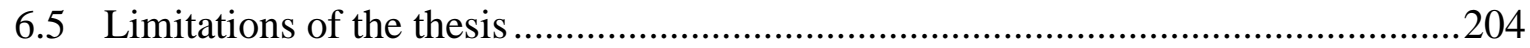

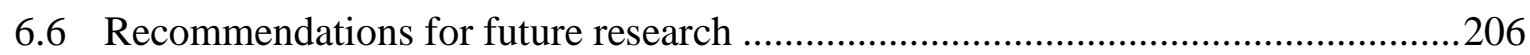

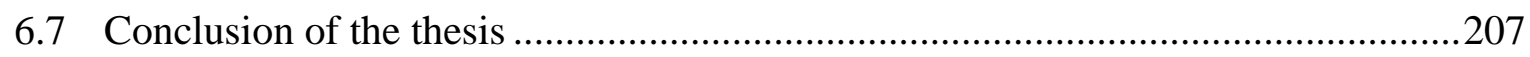

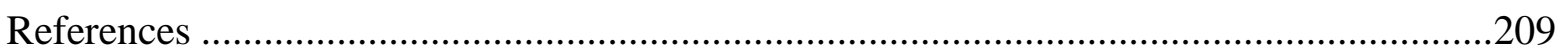

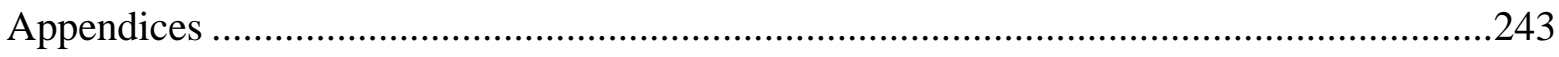

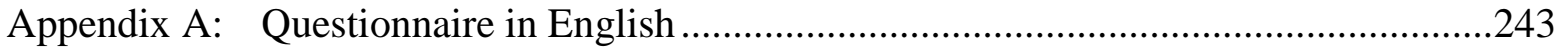

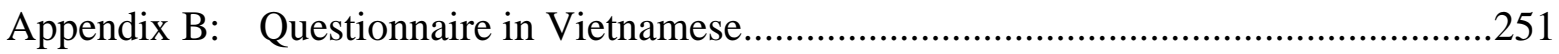

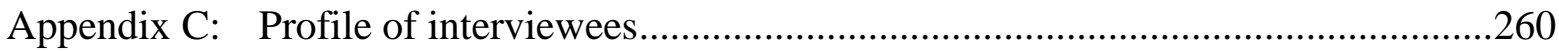

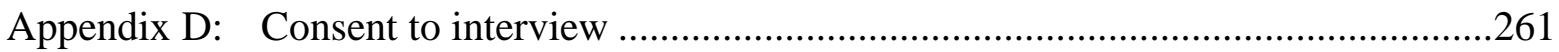

Appendix E: Semi-structured interview questions for non-managerial employees...........262

Appendix F: Semi-structured interview questions for HR managers ...............................264

Appendix G: Semi-structured interview questions for senior managers ..........................266 


\section{List of Tables}

Table 3-1: Employee PM practices and issues in developing countries. .61

Table 4-1: Measures of variables.

Table 4-2: Demographic profile of survey respondents

Table 4-3: Cut-off points for determining model fit

Table 5-1: Factor loading of the five employee PM practices 120

Table 5-2: Results of CFA for the employee PM practices

Table 5-3: Factor loading of the contextual factors

Table 5-4: Results of CFA of the contextual factors

Table 5-5: Factor loading of the cultural factors

Table 5-6: Results of CFA of the cultural factors

Table 5-7: Factor loading of the intervention practices

Table 5-8: Results of CFA of the intervention factors

Table 5-9: Factor loading of the outcome effectiveness variables

Table 5-10: Results of CFA of the outcome effectiveness factors

Table 5-11: Correlation of constructs and the squared root of AVE. 126

Table 5-12: List of organisations with respondents segmented into clusters 129

Table 5-13: Mean value of outcomes across three clusters 130

Table 5-14: Multinomial regression of contextual variables 132

Table 5-15: Indirect effects of contextual factors

Table 5-16: Direct and indirect effects of the modified model 136

Table 5-17: Results of moderated regression analysis for nepotism.... 138

Table 5-18: Results of moderated regression analysis for personal relationships 138

Table 5-19: Results of moderated regression analysis for communication 141

Table 5-20: Results of moderated regression analysis for training ..... 141 
Table 6-1: Summary of the research findings .170 


\section{List of Figures}

Figure 3-1: Conceptual framework of the development of ................................................ 83

Figure 4-1: Research process for the convergent parallel design ......................................90

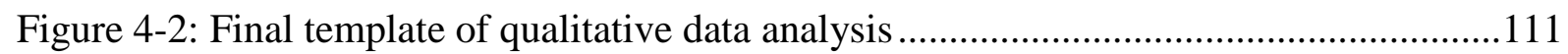

Figure 5-1: Mean values of five PM practices across the three clusters..............................128

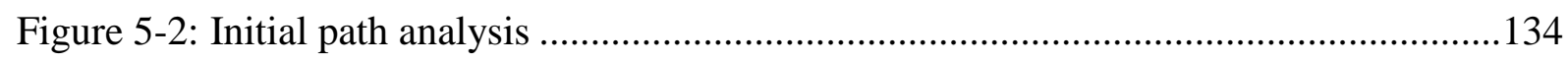

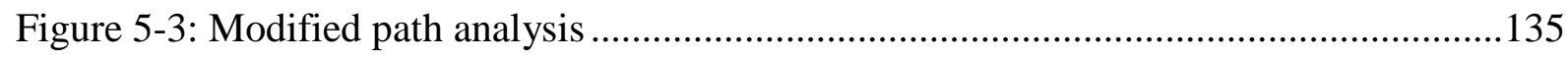

Figure 5-4: Effect of nepotism on the HR autonomy-employee PM relationship.................139

Figure 5-5: Effect of personal relationships on the entrepreneurship-employee PM relationship 140

Figure 5-6: Effect of nepotism on the entrepreneurship-employee PM relationship..............140

Figure 5-7: Effect of communication on the HR autonomy-employee PM relationship........142

Figure 5-8: Effect of communication on the entrepreneurship-employee PM relationship ...142

Figure 5-9: Effect of training on the entrepreneurship-employee PM relationship................143

Figure 6-1: Conceptual framework of the implementation of ............................................194 


\section{Main abbreviations and acronyms}

AMOS

ANOVA

ASEAN

AVE

CFA

CFI

CPV

DOHA

EU

FDI

GDP

GFI

GSO

HR

HRM

ISOC

KPI

MARD

MBO

MOHA

MOJ

NPM

OECD

OPM

PA

PAR

$\mathrm{PM}$

PPC

SMART

PCI

RMSEA

SPSS
Analysis of Moment Structures

Analysis of Variance

Association of Southeast Asian Nations

Average Variance Extracted

Confirmative Factor Analysis

Comparative Fit Index

The Communist Party of Vietnam

Department of Home Affairs in Provinces

European Union

Foreign Direct Investment

Gross Domestic Product

Goodness of Fit Index

General Statistics Office of Vietnam

Human Resource

Human Resource Management

The Vietnam Institute for State Organisational Sciences

Key Performance Indicator

Ministry of Agriculture and Rural Development

Management by Objectives

Vietnam Ministry of Home Affairs

The Vietnam Ministry of Justice

New Public Management

Organisation for Economic Cooperation and Development

United States Office of Personnel Management

Performance Appraisal

Public Administration Reform

Performance Management

Provincial People's Committee

Specific, Measurable, Appropriate, Realistic and Time-Bound

Provincial Competitiveness Index

Root Mean Square Error of Approximation

Statistical Package for Social Science 
The U.S

UNDP

USD

VCCI

VIF

VND
The United States

The United Nations Development Programme

United States Dollar

Vietnam Chamber of Commerce and Industry

Variance Inflation Factor

Vietnamese Dong (the currency of Vietnam) 


\section{CHAPTER 1. INTRODUCTION}

\subsection{Background of study}

Public administration reform (PAR) has become a mainstream activity of many governments during the last three decades (Farazmand 2002; Cheung, Jones et al. 2013). Globalisation, ageing populations, budget constraints and increasing demands for quality services have forced governments to continuously innovate operational modes and managerial practices aiming to "do more with less" while better satisfying citizens" needs (Arnaboldi, Lapsley et al. 2015). For transitional countries such as Eastern European countries, China and Vietnam, PAR is regarded as indispensable in the transformation from a centrally-planned to a market economy (Painter 2003).

In each country, given distinct conditions and development levels, PAR may take several forms, including institution building, decentralisation, restructuring, privatisation and civil service reform. However, PAR frequently targets administrative capacity building and employee performance improvement (Farazmand 2002; Jacobs 2004; Ohemeng, Zakari et al. 2015) because employee performance is central to enhancing the quality of public service (Ingraham and Kneedler 2000). The need to manage employee performance is even more urgent in developing countries because employee passivity, irresponsibility, corruption, poor performance and low work ethic often make economic reform programmes, as well as social development goals, less fruitful and open to the risk of failure (Taylor 1992; Randma-Liiv 2005; Berman, Bowman et al. 2015; Ho and Im 2015). Though past employee performance reforms have targeted selection, training, appraisal and compensation, agreement exists that the above employee problems have not significantly improved (Tong, Straussman et al. 1999; Jacobs 2004; Randma-Liiv 2005; Poon, Hung et al. 2009; Berman 2015). Enhancing employee performance has proven difficult in developing and developed countries alike (Jacobs 2004; Holloway, de Waal et al. 2009; Burns and Zhiren 2010; West and Blackman 2015).

To date, performance appraisal has been considered as one of the most frequently used tools for managing employee performance, but practitioners usually claim that appraisal systems do not really work effectively. Performance appraisal (PA) is defined as a process of evaluating individuals' behaviour and accomplishments in the past and present to differentiate between members in the organisation. The primary purpose of PA is to arrive 
at objective administration decisions such as selection, promotion, transfer, remuneration, dismissal and so on (Heyel 1958; DeVries, Morrison et al. 1981; Bratton and Gold 2012). However, irrespective of a great amount of time and effort spent by researchers, traditional PA still encounters persistent defects such as cognitive biases, including lenient and central tendency errors, and the political motivation of raters (Bretz Jr, Milkovich et al. 1992; Longenecker and Gioia 2001; Merritt 2007; Gardner 2008). When perceived as a threatening and punitive tool, performance appraisal tends to lower creativity and demotivate rather than motivate employees (Law 2007). Hence, poor PA may create more problems than it solves (Thomas and Bretz Jr 1994).

Although it is worth taking into account these limitations, advocates of PA maintain that organisations with successful PA programmes outperform organisations without such programmes in terms of profits, productivity, growth and development (Bouckaert and Halligan 2007; Dessler 2008). In fact, PA is still one of the universal elements in management (Law 2007; Lawler, Benson et al. 2012). Therefore, instead of abandoning these elements, we should find ways to improve them because if properly designed and implemented, they can make a big contribution to organisational effectiveness (Pulakos and O'Leary 2011; Lawler, Benson et al. 2012).

The need to improve traditional PA led to the birth of employee PM in the 1970s (Bretz Jr, Milkovich et al. 1992; Armstrong and Baron 2005; Kinicki, Jacobson et al. 2013). As discussed in detail in Chapter 3, employee PM is defined as "a continuous process of identifying, measuring and developing the performance of individuals and teams and aligning performance with the strategic goals of the organisation" (Aguinis 2009, p.2). To date, there is "no articulated consensus on what the main components of PM are" (Schleicher, Baumann et al. 2018 p.2211). However, the process of employee PM usually involves setting goals, appraising performance, providing feedback and coaching, encouraging participation, providing rewards for good performers, and handling poor performers (Shafie 1996; Lawler 2003; Luecke and Hall 2006; Shields 2007; Aguinis 2009; Pulakos and O'Leary 2011; Tung, Baird et al. 2011; Haines III and St-Onge 2012; Kinicki, Jacobson et al. 2013). Employee PM supposedly improves individual attitudes and motivation, and subsequently organisational performance (De Waal and Gerritsen-Medema 2006; Aguinis 2009; DeNisi and Sonesh 2011). Besides, employee PM often aims to promote result-driven cultures, responsibility, accountability, transparency and efficiency to 
meet the organisation's strategic goals (Otley 1999; De Waal 2007; Decramer, Smolders et al. 2012). Arguably, employee PM is becoming more and more critical in the context of cost reduction and increasing public expectation (West and Blackman 2015).

Compared to traditional PA, PM has some fundamental differences. Perhaps the most notable difference is the ultimate purpose. The primary purpose of PA is to provide information for administrative decisions by distinguishing between members within an organisation according to their past and present performance. Meanwhile, employee PM is carried out to improve employee and organisational performance in the future (Lebas 1995; Armstrong and Baron 1998; Aguinis 2009). In addition, it is noteworthy that PA is still a substantial part of the performance management system that consists of a number of interconnected practices (Lebas 1995; Beardwell and Claydon 2007; DeNisi and Murphy 2017).

The adoption of employee PM has been accelerated by the movement of New Public Management (NPM) (Vallance 1999; De Waal 2007), a doctrine emphasising "market-type managerial methods", "explicit standards of performance", "greater emphasis on output controls", and "greater competition in the public sector" (Hood 1991, p.4,5). For the last twenty years, 93.3 percent of OECD member countries have implemented PM systems as a management tool to increase quality and efficiency in public service delivery (Lah and Perry 2008). In a growing trend, many developing countries have eagerly introduced or are planning to adopt employee PM as a radical solution to solve persistent HR problems e.g. China (Liu and Dong 2012), Malaysia (Shafie 1996), Ghana (Ohemeng, Zakari et al. 2015), Maldives (Asim 2001), Thailand (Koonmee 2009), Estonia (Randma-Liiv 2005).

Along with the pervasive adoption of employee PM, the past three decades have seen an explosion of research in this field (Fletcher 2001; Manoharan, Muralidharan et al. 2009; Lee 2017; Ohemeng, Amoako Asiedu et al. 2018; Schleicher, Baumann et al. 2018). This proliferation has not yet shown signs of decline. One of the most frequently mentioned topics is how to enhance the effectiveness of employee PM practices such as goal setting, feedback, evaluation, reward, coaching and participation (Cleveland, Morrison et al. 1986; Roberts and Reed 1996; Lawler and McDermott 2003; Roberts 2003; Latham and Locke 2007). Besides, employees' perceptions and the consequences of introducing employee PM have been widely studied (Erdogan 2003; Hope Sr 2003; Chang and Hahn 2006; Brown, Hyatt et al. 2010; Kim 2014; Mizrahi and Minchuk 2017). 
Contextual factors around the implementation of employee PM practices have recently attracted attention from scholars (Wood and Marshall 2008; Haines III and St-Onge 2012; Gorman, Meriac et al. 2017; Schleicher, Baumann et al. 2018). There has been a growing perception that employee PM should be viewed as a social and communication process rather than primarily as a measurement tool (Murphy and Cleveland 1995; Fletcher 2001; Haines III and St-Onge 2012). The need for research on contextual factors has become even more urgent in developing countries when practitioners unexpectedly discover that many highly successful programmes in industrialised countries fail in their country where sociocultural and institutional differences exist (Mendonca and Kanungo 1996; Aycan, Kanungo et al. 2000). So far, a wide range of contextual factors from macro-level variables (e.g. history, law, culture, economic conditions, and political environment) to micro-level variables (e.g. the type of tasks, organisational size, task characteristics and management concerns) has been acknowledged (Murphy and Cleveland 1995; Hofstede 2007; Ryu 2010; Wadongo and Abdel-Kader 2014). Arguably, the list of potential contextual variables may not be complete (Marshall and Wood 2000; Wood and Marshall 2008).

In addition to the aforementioned topics, there is increasing interest in the actual effects of employee PM on employee and organisational performance (DeNisi and Murphy 2017). It has been said that, unlike performance appraisal done mostly for administrative decisions, PM should be assessed by its capacity to improve individual performance and organisational performance (Haines III and St-Onge 2012; Abbas 2014). Accordingly, some research has started investigating real effects, rather than the "rhetoric" of employee PM. For instance, Taylor and Pierce (1999) investigated the impact of introducing employee PM on employees' efforts. Rodgers and Hunter (1991) studied the effect of goal setting, participation and feedback on organisational productivity. Meanwhile, Berberian (2008), Hasnain, Manning et al. (2012) and Taylor and Beh (2013) discovered the effect of reward programmes on employee job satisfaction and performance.

\subsection{Statement of the problem}

Despite the popularity of employee PM, its successful implementation is still a global challenge even in the most developed countries, where the failure rate has been estimated to be approximately 60 percent (Holloway, de Waal et al. 2009; Haines III and St-Onge 2012; Carassus, Favoreu et al. 2014). In reality, regardless of a large amount of research devoted to employee PM (Lawler and McDermott 2003; Thomas 2007), "the formula for effective 
performance management remains elusive" (Pulakos and O'Leary 2011 p.1). Davis (2015) reported that current research lacks the ability to "identify consistent factors or practices that most positive affect employee performance" (p.2). Irrespective of successive efforts devoted to improving such managerial instruments, negative outcomes still occur (Azzone and Palermo 2011). In fact, the majority of HR professionals perceive that their employee PM systems are not effective in improving organisational performance (Haines III and St-Onge 2012). Employees and supervisors often dislike employee PM as well and see it as a control mechanism or an unnecessary administrative burden (Thomas and Bretz Jr 1994; Biron, Farndale et al. 2011; Kim 2014). Some research has even reported that the introduction of employee PM not only has limited impact on performance improvement but also causes decreased productivity as well as other unwanted consequences, including the distortion of performance data (Risher 2011; Stanton and Nankervis 2011; Mustapha and Daud 2012; Richard, Plimmer et al. 2015; Rubel and Kee 2015; Mizrahi and Minchuk 2017).

The reasons as to why employee PM systems often fall short of expectations have become a common concern in the literature (Longenecker 1997; Ohemeng 2009; Pulakos and O'Leary 2011). Many reasons have been suggested. First, it has been said that the research is mostly theoretical or based on a laboratory environment or simulations, so the results might not capture the complexity in real work environments (Bretz Jr, Milkovich et al. 1992; Fletcher and Williams 1996; Walsh 2003; Kim 2014). A number of researchers also concur that, despite no shortage of theoretical research on the "surface rhetoric" about employee PM, there is a lack of empirical research into the "actual practices" that can enhance its effectiveness (Pollitt 2005; DeNisi and Pritchard 2006; Biron, Farndale et al. 2011). Some attribute the low rate of successful PM schemes to lack of knowledge about the specific PM practices that are crucial for effective implementation (Lawler 2003; DeNisi and Pritchard 2006; Posthuma and Campion 2008; Pulakos and O'Leary 2011). Additionally, it has been said that the existing research mostly addresses employee PM practices in isolation without discussing them as the entire process of integrated practices (DeNisi and Murphy 2017). The lack of robust guiding theories usually causes managers to be confused or even misleading in the implementation process (Lawler and McDermott 2003; Cheng, Dainty et al. 2006).

Second, arguably, many employee PM systems fail because they only focus on technical issues, without taking contextual factors into consideration (Marshall and Wood 
2000; Stoskopf 2002; Haines III and St-Onge 2012). Many scholars have agreed that the effectiveness of human resource management (HRM) practices must be congruent with the social-cultural context in which they are conducted (Mendonca and Kanungo 1996; Aycan 2005; Ohemeng 2009). However, the literature still lacks a clear and cohesive understanding of the degree, pattern, and circumstances in which contextual variables affect employee PM (Murphy and Cleveland 1995; Decramer, Smolders et al. 2012; Pichler 2012).

In addition to these common challenges, the adoption of employee PM in the public sector in developing countries is regarded as even more challenging. Public managers in these countries encounter not only difficulties regarding the development of measurable criteria, union opposition, limited autonomy and inadequate government funding (Marshall 1998; Verbeeten 2008; Bowman 2010; Taylor and Beh 2013), but also obstacles inherent in the developing context. There is scepticism about the applicability of employee PM in developing countries because the western context where it was first innovated and has evolved is different from developing countries economically, culturally, legally and politically (Mendonca and Kanungo 1996; Vallance 1999; De Waal 2007; Ho and Im 2015; Ohemeng, Amoako-Asiedu et al. 2018). Specifically, flawed legal systems, poor management capacity and traditional cultural characteristics have constrained the effectiveness of employee PM systems, even the best technically designed systems. Unfortunately, research on the implementation of employee PM in developing countries is relatively rare (Yeganeh and Su 2008; Jankulović and Škorić 2013; Ohemeng, Zakari et al. 2015). Only 5 percent of empirical studies are devoted to developing countries while up to 95 percent of empirical studies are conducted in developed contexts (Farashahi, Hafsi et al. 2005).

All these problems together explains why regardless of the proliferation of employee PM reforms, successful schemes, particularly in developing countries, are still very limited (Shafie 1996; Randma-Liiv 2005; Koonmee 2009; Liu and Dong 2012; Ohemeng, Zakari et al. 2015).

\subsection{Research purposes}

This study was conducted to narrow the gap in the literature on employee PM by investigating methods and factors strengthening its effectiveness in developing contexts. Specifically, this study has five main purposes. First, it examines whether the adoption of five employee PM practices, goal-based appraisal, feedback, reward-for-performance, 
addressing poor performers and employee participation in concert, can improve employee attitudes and organisational performance. To date, although the relationships between employee PM and its outcomes have been discussed in the literature, many have been tested in laboratory settings or have other limitations such as depending on small interview samples and being conducted in developed contexts. Some quantitative research has been done but most only investigates the effect of individual employee PM practices (Verbeeten 2008). Examples include pay-for-performance (Taylor and Beh 2013), feedback (Jawahar 2006) and appraisal (Oh and Lewis 2009). The results of this research can provide empirical evidence to contribute to the contemporary debate as to whether employee PM practices, in combination, is applicable or effective in inducing desired outcomes in the public sector in developing countries (Mendonca and Kanungo 1996; Schick 1998; De Waal 2007; Verheijen and Dobrolyubova 2007).

Second, this research responds to the call to pay more attention to contextual factors that relate to the implementation of employee PM in developing countries. Specifically, it aims to shed light on the effect of three institutional factors (accountability, entrepreneurial leadership, and HR autonomy), and one capacity factor (HR competence) on the development of employee PM. To date, institutions, capacity and culture have been perceived as contextual factors influencing the effectiveness of employee PM in developing countries. Nonetheless, very few studies have investigated specific factors as well as through what patterns by which these factors affect the implementation of employee PM (Jankulović and Škorić 2013; Ohemeng, Zakari et al. 2015).

Third, through this research, the author wants to extend the understanding of the effects of interpersonal relationships and nepotism on the implementation of employee PM. Culture and other traditional values have been identified as one of the fundamental problems that have constrained and continue to constrain the successful implementation of PM systems in developing countries (Ohemeng 2009). There is a view that management practices such as employee PM developed in individualistic and low power distance cultures may not be suitable in a society characterised by collectivism, high power distance and relationship-orientation. In such a society, direct feedback is understood as attacking the person's personality and may destroy the harmony that is expected to govern interpersonal relationships (Hofstede 1998). To date, in spite of no shortage of studies on the effect of cultural factors, most of them rely on the cultural dimensions proposed by Hofstede (1998) 
such as power distance, individualism vs. collectivism, masculinity vs femininity and uncertainty avoidance. Meanwhile, interpersonal relationships and nepotism are still underresearched, despite their prevalence in developing countries. In the article "Performance appraisal and performance management: 100 years of progress”, DeNisi and Murphy (2017) conclude that "we need more research on the effectiveness of individual-level performance management techniques in different cultures" (p.429).

Fourth, with the purpose of seeking effective intervention practices in the implementation of employee PM, this research examines whether communication and training can strengthen the development of employee PM. The result of this investigation will be significant because there are arguments that the failure of employee PM implementation is regularly attributed to the absence of intervention practices (Roberts 2003; Biron, Farndale et al. 2011; Lawler, Benson et al. 2012). So far, although communication and training have been suggested as effective tools in organisational change (Elving 2005; Fernandez and Rainey 2006), there is a dearth of empirical research that investigates the actual effects of these practices in employee PM implementation.

Finally, based on the research results, the thesis aims to provide suggestions and implications for practitioners and policy-makers in developing countries in general, and Vietnam in particular. Accordingly, practitioners may have a more comprehensive insight into how to design and implement an effective employee PM system. Meanwhile, policymakers may realise which pre-conditions or strategic solutions are needed to promote employee PM reform.

\subsection{Research questions and hypotheses}

Based on the literature review of employee PM in developing contexts and the research purposes discussed above, this research addresses five main research questions:

Question 1: Does employee PM in developing countries enhance desirable outcomes such as employee motivation, organisational commitment, job satisfaction and organisational performance?

Question 2: Which contextual factors drive the development of employee PM?

Question 3: Can employee PM be a mediating factor for the effect of contextual factors as reform efforts on desirable outcomes? 
Question 4: How do cultural variables such as interpersonal relationships and nepotism hinder the development of employee PM?

Question 5: What intervention practices can be introduced to strengthen the development of employee PM?

Based on these research questions, this research reviewed relevant theories and research in relation to the developing context in order to develop five corresponding hypotheses. For example, Hypothesis 1 was developed based on goal-setting, expectancy, equity and social-exchange theories and the evaluation of recent employee PM reforms in developing countries. Hypothesis 2 was formulated based on personal experience, results from preliminary interviews and a meticulous review of the relevant literature. Hypothesis 3 was developed based on the combination of hypotheses 1 and 2. Meanwhile, Hypotheses 4 and 5 were based on the research questions, literature review and personal observation of the influence of these factors.

Hypothesis 1: Employee PM is associated with increased perceptions of employee motivation (H1a), organisational commitment (H1b), job satisfaction (H1c) and organisational performance $(\mathrm{H} 1 \mathrm{~d})$.

Hypothesis 2: Agency accountability (H2a), HR autonomy (H2b), entrepreneurial leadership $(\mathrm{H} 2 \mathrm{c})$ and $\mathrm{HR}$ competence $(\mathrm{H} 2 \mathrm{~d})$ are associated with the development of employee PM practices.

Hypothesis 3: Agency accountability (H3a), HR autonomy (H3b), entrepreneurial leadership (H3c) and HR competence (H3d) indirectly affect employee motivation, organisational commitment, job satisfaction and organisational performance through the mediation role of employee PM.

Hypothesis 4: The relationships between the contextual factors and employee PM are moderated (weakened) by the cultural variables of nepotism $(\mathrm{H} 4 \mathrm{a})$, and interpersonal relationships $(\mathrm{H} 4 \mathrm{~b})$.

Hypothesis 5: The relationships between the contextual factors and employee PM are moderated (strengthened) by communication (H5a), and training (H5b).

\subsection{Research setting}

As a quickly transiting economy, Vietnam provides an appropriate case setting for this study. Thirty years after major economic reform, the Vietnam civil service is generally 
described as having a low work ethic, opportunism, irresponsibility, wastefulness and arbitrary decision-making (Poon, Hung et al. 2009; CPV 2016). Vietnam shares many problems with other transitional and developing countries, such as poor employee performance, systematic corruption, low efficiency, "red tape", and abuse of power (Painter 2003; Mau, Tham et al. 2012; Vian, Brinkerhoff et al. 2012; Pham 2018).

In response, some public agencies have implemented new strategies for effectiveness, including new schemes for performance appraisals and employee PM. To support this process, the government has embraced several PAR initiatives, including permitting public service delivery organisations to have increased autonomy and accountability (Vietnam 2002; Vietnam 2005). Accordingly, self-financing service delivery organisations were allowed to have more autonomy in expenditure and personnel decisions such as recruitment, promotion and income distribution. In addition, some provinces and central ministries are permitted to introduce pilot programmes or experiments in HRM practices, including performance-based appraisal, competitive examinations for promotion, pay-forperformance, and anonymous online feedback. Such programmes were known as "fencebreaking" mechanisms because they were only applied to some pilot agencies and in a certain period. While the PM reform efforts are not yet mainstream and only a small proportion reached a relatively advanced degree of development, they do provide the opportunity to examine whether these various changes can lift employee PM in these agencies. At the same time, it is worth keeping in mind that, the majority of public organisations were still adhering to obsolete employee PM systems that mostly rely on annual evaluation with abstract criteria such as political quality and loyalty, collective feedback and seniority-based pay.

In addition to the above features, the Vietnam public sector possesses many of the same characteristics as other developing countries such as weak institutions, corruption and limited capacity. It is also characterised by many traditional values such as Confucianism, relationship-orientation, paternalism, fatalism and nepotism. All these factors make Vietnam a suitable case study for studying employee PM in the developing context.

\subsection{Research methodology}

This study adopts a pragmatic approach by deliberately harmonising two traditional research perspectives, namely positivism and social constructionism. This approach argues that all measurement is fallible and all observations are theory-laden. Therefore, we need to 
use triangulation to get a better understanding of what is happening in reality (Trochim and Donnelly 2001). Accordingly, both quantitative and qualitative research methods, as a mixed methods approach, will be used to capture the advantages of each method as well as overcome the inherent shortcomings of each methodology (O'Leary 2013).

The mixed methods approach is well suited to this study. The quantitative strand allows the researcher to predict trends and relationships between the studied variables. Specifically, it helps to predict whether the adoption of advanced employee PM systems is able to lead to desirable outcomes as well as identify what contextual factors relate to the development of employee PM. Such findings are usually useful for policy-makers (Easterby-Smith, Thorpe et al. 2012). On the other hand, the qualitative strand gives an insight into why employee PM practices, in combination, can induce the desired outcomes while individual PM practices can only have limited effects. Similarly, interviewees' perception can give readers a better understanding of why and how contextual factors including institutions, capacity and culture can influence the development of employee PM.

As part of the mixed methods approach, this research adopted the concurrent triangulation design for collecting, analysing and integrating data. This choice aimed to minimise the dephasing between qualitative and quantitative strand caused by changes in each organisation. Also, it enabled the researcher to save time and enhance the validity of findings (Creswell 2009)

With respect to data collection, the study consisted of (a) 30 interviews and document analysis and (b) a quantitative survey $(n=322)$. The participants in this study included senior managers, line managers, HR managers and employees working in 29 diverse organisations in the Vietnamese public sector. As relatively few organisations have experimented and developed advanced employee PM, sampling is deliberately purposive in order to include organisations with different degrees of PM development; the purpose is to ensure a range of employee PM practices, rather than a representative sample of organisations or public servants. However, this research also sought to ensure that the sample includes important and diverse public sector characteristics, such as central vs local organisations; administration agencies vs service providing institutions; and large vs small ones.

The analytical methods followed the research questions of this study. For the quantitative analysis, all data were analysed with the aid of the Statistical Package for Social Science (SPSS) computer programme, version 23.0 and the Analysis of Moment Structures 
(AMOS), version 25. To address the first research question about whether employee PM can lead to the desirable outcomes, the research used cluster analysis to group the employees according to the development levels of PM practices they experienced. One-way analysis of variance (ANOVA) was then used to examine the impact of different PM development levels on employee attitudes and perceived organisational performance. Next, multinomial logistic regression was used to address the second research question about the effect of the contextual factors on the development of employee PM. For the third research question, structural equation modelling (SEM) was adopted to test the mediating effect of employee PM on the relationships between the contextual factors and the outcome variables. For questions 4 and 5, the research used three-step linear regression (Zedeck 1971) to examine the moderating effects of the cultural variables and the intervention variables on the development of employee PM. Finally, the quantitative analysis findings were triangulated against the qualitative data collected from semi-structured interviews and managed by NVivo, a software package for qualitative data analysis.

Validity and reliability in this study were furthered in several ways. With regard to the quantitative data, existing scales with high internal reliability were used whenever possible. Then these initial measures were piloted with experienced HR professionals to ensure that the measures were valid and compatible with the study's setting. Once the instrument was developed, one more pilot test was carried out to revise ambiguous questions and remove repetitions, as well as providing an initial evaluation of the internal reliability of constructs.

For the qualitative data collection, a semi-structured interview format (an interview protocol) was used to increase the validity and reliability of the interview data. Interviews were conducted with different groups such as senior managers, HR professionals and employees to triangulate the collected data. Simultaneously, to encourage the interviewees to speak out about their perceptions honestly and candidly, the researcher tried to establish an open rapport with the interviewees. The measures included (i) having prestigious people liaise for the interviews, (ii) getting approval from the senior managers, (iii) clearly articulating the purpose of the research, (iv) giving a small gift to each interviewee and (v) using note taking instead of digital recording. The reason for avoiding digital recording is that public employees in Vietnam, as well as some other Asian countries, are often reluctant to speak freely and openly in front of a recorder. All the processes and methods for data 
collection were approved by the Human Ethics Committee of the Victoria University of Wellington.

\subsection{Contributions}

This research contributes to the literature of employee PM, particularly in developing contexts as below.

\subsubsection{Theoretical contributions}

First, the present research finds that PM practices, in combination, improve employee attitudes and organisational performance. Previous studies have shown that employee PM may fail when its components operate in isolation e.g. when appraisal is separated from consequences (De Waal 2007; Liu and Dong 2012); pay-for-performance is not based on objective performance standards (Mwita 2003; Randma-Liiv 2005); or feedback lacks employee input and participation (Chiang and Birtch 2010). For an employee PM system to be effective, it should involve a bundle of practices. This research is one of very few studies that investigate the relationships between employee PM as a bundle of component practices and the outcome variables (Verbeeten 2008). In this regard, Schleicher, Baumann et al. (2018) argue that though employee PM is often viewed as a system of intertwined PM practices, very few studies have so far been conducted in this way.

Second, this study extends our knowledge of contextual factors, focusing on leadership (agency accountability, entrepreneurial leadership), HR autonomy and HR competency. These have been discussed elsewhere, e.g. in policy-making, public administration reform (PAR) and education, but seldom applied to this area and examined in combination (Taylor 1992; Maslowski, Scheerens et al. 2007; Burns and Zhiren 2010; Berman, Chen et al. 2017). To the best of the author's knowledge, this is the first research to examine the effects of these contextual factors on the development of employee PM. The findings provide a greater understanding of the role of contextual factors in the development of employee PM. Specifically, it helps us understand why some public organisations can develop advanced employee PM systems while other organisations tend to engage in laggard PM systems.

Third, research on the relationship between employee PM and organisational performance is not new. However, very few researchers have investigated employee PM as one mediator in the relationship between contextual variables and organisational performance. This study concludes that employee PM could mediate the relationships 
between reform efforts and desired outcomes, including increased employee attitudes and organisational performance. This finding may be meaningful because over the last decades, many reform efforts have been done in developing countries but desirable outcomes are still far from expectations. This research suggests that employee PM might be a missing one that can transform reform efforts to desirable outcomes such as employee performance and organisational performance. This finding consolidates the belief that the effectiveness of PAR activities will be likely limited when lacking an effective employee PM system.

Fourth, this research investigates the negative effects of interpersonal relationships and nepotism on the development of employee PM. Specifically, it indicates that these traditionally cultures constrained the development of employee PM. Although traditional cultures are often mentioned in relation to recruitment and promotion practices, their effects on employee PM tend to be underestimated. These findings provide an in-depth insight into the challenges of introducing employee PM in developing countries.

Finally, the research provides a theoretical model of implementing employee PM in the public sector in developing countries based on the analysis results. Perhaps for the first time, contextual factors, the implementation of employee PM and desirable outcomes are incorporated into a theoretical model. This model provides scholars a broader insight into the implementation of employee PM in the public sectors in a developing context. Therefore, future researchers can utilise or test this model, fully or partially, in other developing contexts

\subsubsection{Empirical contributions}

This research is one of the very few pioneering studies that quantitatively examine the relationships between employee PM and the outcome variables. Although these relationships have been discussed in an enormous amount of theoretical research (Ohemeng, Amoako-Asiedu et al. 2018), empirical studies are very limited, particularly in developing countries. The findings from this research shed light on the debate regarding the applicability of employee PM in the public sector (Verbeeten 2008) and in developing contexts (De Waal 2007). Results indicate there are significant and positive relationships between the development of employee PM and employee attitudes and organisational performance. In other words, they affirm that employee PM can be effective across various settings, though the specific ways in which PM practices are embraced may and should vary across settings (Schleicher, Baumann et al. 2018). 
Second, examination of the role of intervention practices shows that communication and training significantly strengthen the effect of the contextual factors on the development of employee PM. This finding is important because, although the role of these practices has been discussed in the literature, the extant research is usually theoretical and most have been conducted in the developed context. This research not only confirms the positive effects of intervention practices but also suggests ways in which they can be effectively implemented in developing settings.

\subsection{Limitations of the research}

Like all studies, this study has limitations. A theoretical limitation is that the research may not account for all the complexity and multidimensionality of employee PM. Although the research covers five dimensions of this construct, it does not include some other important aspects such as coaching and development. This is because while coaching and development may be popular in developed countries, they are rarely applied in Vietnam, at least in the public sector.

Second, no study can examine all factors. While this study examines perceived accountability, HR autonomy, entrepreneurial leadership, HR competence, nepotism and personal relationships, other factors such as other traditional cultures, job type, trade union, organisational scale remain unexplored and need further research.

Third, the findings from this research may not sufficiently represent organisations in the Vietnamese public sector because some organisations that use advanced employee PM in this study are to some degree self-selected as they elected to pursue this reform. In fact, the organisations engaged in advanced employee PM in Vietnam are sparse. The majority of public organisations in Vietnam still adhere to traditional, trait-based appraisal. Therefore, a selective sample is necessary because the main purpose of this research is to investigate the factors driving the development of employee PM rather than seek generalisation.

Fourth, because all the independent and dependent variables in this research were collected at the same time, some quantitative analysis results only indicate the correlations between variables rather than causal relationships. However, utilising multinomial logistic regression and triangulating from the qualitative data can consolidate the validity of the conclusion about causal directions. 
Fifth, this study's measures are necessarily based on perceptions, as objective data (such as job attitudes and motivation) are not available (Podsakoff and Organ 1986), and other sources of objective data such as the organisational performance of public organisations are also unavailable in Vietnam. However, the consistency of these results across the clusters of organisations, triangulated with qualitative interviews, provides important validity checks.

Sixth, this research did not include employee performance as one of the most expected outcomes of introducing employee PM. The reason is that directly asking respondents about their performance may lead to biased responses due to social desirability. Though some relevant variables such as motivation, organisational commitment and job satisfaction were used as substitutional variables, they may not represent employee performance sufficiently. Future research should address this limitation by including responses from the respondents' supervisors in evaluating employee performance.

Finally, because this study was conducted in Vietnam, which may have a different political and cultural context compared to many other countries, generalization of these findings should be done with caution.

\subsection{Organisation of the thesis}

This section presents how the thesis is organised according to chapters and the main content of each. The thesis is structured into six chapters. Following the introductory chapter is the chapters as below:

Chapter 2 Background context: provides background to the context of the Vietnamese public sector where data for this research was collected. The main content in this chapter includes: (i) an overview of the history of Vietnam and PAR programmes, (ii) current HRM policies in the public sector and the current state of employee performance, (iii) traditional appraisal and innovation towards employee PM.

Chapter 3 Literature reviews: reviews the literature on employee PM in transitional and developing countries. This chapter comprises seven sections: (i) public administration reform in transitional countries; (ii) evolution and definition of employee PM; (iii) the trend to adopt employee PM practices in developing countries; (iv) desirable outcomes of employee PM and supporting theories; (v) contextual factors; (vi) intervention practices and (vii) a conceptual framework. 
Chapter 4 Research methodology: presents the research approach and design. It begins by explaining the selection of paradigm, research approach and research design, and then discusses how the data were collected, analysed and integrated. Next, it presents the measures applied to establish research quality. The final sections discuss the ethical considerations and the limitations of the research methods.

Chapter 5 Results: reports the results of the quantitative and qualitative analysis. Accordingly, it presents the results of examining the hypotheses by statistical analyses and the results from the qualitative data analyses according to the research themes. The two strands are then compared and contrasted in the integration section.

Chapter 6 Discussion, implications and conclusions: discusses the findings presented in Chapter 5 in relation to the literature and the existing debates. Based on these findings, it outlines implications for policy-makers, practitioners and researchers. This is followed by a discussion about the contributions and limitations before putting forth recommendations for future research.

\subsection{Summary of Chapter 1}

This chapter provides an introduction and outlines the major contents of the entire thesis. Accordingly, this research aims to narrow the gap in the literature by investigating the implementation of employee PM in the public sector in a developing context. By analysing quantitative and qualitative data collected from 29 public organisations in Vietnam as a transitional country, this research has some important contributions. First, the findings of the research revealed that regardless of inherent challenges, employee PM is able to induce desirable outcomes in developing contexts. Second, for an employee PM system to be effective, the employee PM practices need to be well designed and implemented in combination rather than in isolation. Third, this study extends our knowledge of context by examining the role of agency accountability, entrepreneurial leadership, HR autonomy, the competence of HR units and the cultural variables in the success of employee PM. Finally, the research contributes to the literature by investigating the moderation effect of communication and training on the development of employee PM. These results not only make significant contributions to the field of employee PM but are also relevant to other public administration reforms in developing countries. 


\section{CHAPTER 2. BACKGROUND CONTEXT}

This chapter discusses the implementation of employee PM in the context of the Vietnamese public sector. First, it provides an historical overview and then presents the government's efforts to reform public administration as a necessary requirement to transform to a market economy. After that, it reports ongoing HRM practices before evaluating the current state of the contingent of employees working in the public sector. This section is followed by a discussion of the existing appraisal practice and changes towards employee PM. The final section summarises the chapter.

\subsection{Historical background}

Vietnam has a long-standing history of more than 2000 years (from 669 BC to 1858 AD) under feudal dynasties. The most notable feature throughout Vietnamese history is continuous wars against foreign invaders. The country experienced over 1000 years (from 111 BC to 939 AD) under Chinese rule. In 939 AD Ngo Quyen was victorious against the Chinese and this opened an enduring independent period for the nation although it still witnessed continuous civil wars. Independence lasted until 1858 when the French invaded and established a semi-feudal colonial regime for one century (from 1858 to 1954). With their defeat at the battle of Dien Bien Phu, the French officially withdrew from Vietnam in 1954 (Zhu, Warner et al. 2007; Tuyen 2013). However, with the signing of the Geneva Accord, the country was temporarily divided into two parts at the $17^{\text {th }}$ parallel.

While South Vietnam was supported by America to develop a capitalist market economy, North Vietnam (the Democratic Republic of Vietnam), led by President Ho Chi Minh, embraced socialism with the priority goal to reunite the country. Like other socialist nations at that time, North Vietnam adopted the Soviet-style centrally planned economic model that was characterised by sole public (state and collective) ownership. Accordingly, all main means of production belonged to the state or cooperatives, and the decisions on what to produce, how to produce and for whom were centrally made by administrative orders from the central government (Zhu, Warner et al. 2007; Tuyen 2013). Despite inherent limitations, the subsidised and centrally planned economic model was seen as a necessity for North Vietnam to mobilise resources for the goal of reuniting the country.

After the country was reunified in 1975, the North continued to establish the public ownership regime in the South by nationalising factories, assets, land and other production 
means from the capitalists of the old regime. However, the faulty policies derived from the state and collective ownership economic models, coupled with the sudden reduction of foreign aid due to the collapse of socialist nations in Eastern Europe, caused the Vietnamese economy to fall into a severe economic crisis in the 1980s. Food was extremely scarce. Average inflation during 1986-1988 was 402.1 percent (Ngoc 2011). Facing this critical time, with the well-known slogan "Doi moi hay la chet", or "reform or death", the Congress VI of the Central Committee of the communist party of Vietnam (1986) made a momentous and historic decision that the country would move from a centrally planned to a socialistoriented market economy. Nowadays, this decision is still called the "renewal", "renovation", or "doi moi" policy. Through the policy of renovation, from one of the world's poorest countries with GDP per capita of USD 140 in 1993, Vietnam became a lower middle-income country in 2008 with GDP per capita of USD 1000. This increased to USD 2215 in 2016 (Diep 2012; Bach 2016). Thanks to the high economic growth rate, the proportion of households in poverty has reduced from 58.1 percent in 1992 to 8 percent in 2013 and 4.5 percent in 2015 (Diep 2012; UNDP 2013; Le 2015).

Although the renewal process has brought remarkable achievements, Vietnam has been facing enormous challenges from a weak and obsolete civil service developed during the century-long colonial period under French rule which emphasised a mandarin structure to rule society rather than a civil service to serve the general public (Poon, Hung et al. 2009). The public service has also been heavily affected by dozens of years under the bureaucratic and central planning mechanism characterised by administrative orders and the mechanism of "beg and give" (Mau, Tham et al. 2012). Undeniably, these historic legacies have influenced service attitudes, management styles, and the operational manner of the public service (Thang and Quang 2005; Zhu, Warner et al. 2007). The current weak public service not only impedes the renovation process, but also possibly makes this process fail (Tung 2013). Therefore, public service quality improvement has been identified as an urgent and fundamental demand for successful transition (Vietnam 2001).

\subsection{Public administration reform programmes}

\subsubsection{Objectives and contents}

Since 1992, with the assistance of international donors, several PAR initiatives have been implemented to improve the efficiency and effectiveness of the public service, thereby facilitating the transition to a market economy. PAR has been identified as one of three 
strategic breakthroughs to help Vietnam to become an industrial and modern country by 2020 (CPV 2011). To date, two particularly comprehensive PAR programmes have been initiated and implemented, namely the PAR Master Programme phase I from 2001-2010 (Vietnam 2001) and the PAR Master Programme phase II from 2011-2020 (Vietnam 2011). The overarching objectives of the programmes are to:

1) develop and perfect the socialist-oriented market economic institution.

2) create an equal, open, favourable and transparent business environment.

3) build an open, clean, professional, modern, effective and efficient administration apparatus.

4) guarantee the exercise of democratic rights of people.

5) develop a contingent of cadres, civil servants and public employees with adequate qualities, capabilities and qualifications to meet the requirements of serving the people and developing the nation (Vietnam 2001; Vietnam 2011).

To achieve these goals, the PAR Master Programme phase I consisted of four components: 1) reforming the constitution, 2) reforming the organisation of the state administrative apparatus, 3) enhancing the quality of the contingent of cadres, civil servants and public employees and 4) reforming public finance. The PAR Master Programme phase II continued with the four preceding components and included another component, namely simplifying administration procedures.

\subsubsection{Results}

Based on the evaluation reports of the government for the periods from 2001-2010 and 2011-2015, the PAR programmes have produced some positive results as briefly summarised below:

1) As a result of institutional reform efforts, approximately 180 laws and 3000 governmental decrees were promulgated or amended to suit the market economic conditions and the demands of international economic institutions (MOHA 2011; Vietnam 2016).

2) 5000 administrative procedures between $2001-2010$ and 4526 administrative procedures between 2011-2015 were reviewed, abolished and simplified. The simplification of administrative procedures, coupled with the expansion of 'one-stop shop', has reduced time and costs for citizens and enterprises (MOHA 2011; Vietnam 2016). 
3) The number of central ministries and agencies under the government has been significantly reduced from 48 to 30 (22 ministries and ministry-level agencies and 8 government agencies e.g. Vietnam news agency, Voice of Vietnam, Vietnam television station and so on). The functions and duties of ministries were also reviewed and refined to avoid overlap and omission. Central ministries have sped up decentralisation so that the provinces have more authority and flexibility to respond to the needs of their localities promptly. Additionally, to enhance management effectiveness, public organisations have been explicitly categorised into administration agencies (making and enforcing policies) and public non-business units (public service delivery institutions). Unlike administration agencies, public non-business units such as universities, hospitals and research institutes can create revenue from providing service to cover costs for their operation. As such, the selffinancing mechanism applying for public non-business units has been formed and gradually perfected. Up until 2015, 25,631 public service delivery institutions nationwide were designated as self-financing organisations. Based on preliminary reports, thanks to the selffinancing mechanism, the public service delivery institutions can use resources such as finances, assets and human capital more effectively, thereby providing better quality services and goods (MOHA 2011; Vietnam 2016).

4) In the period from 2001-2015, 10 million participants have been trained and retrained in modern civil service, including public administration reform, management and leadership skills, professional skills, international integration, foreign languages, information technology and so on. As a result, the knowledge and skills in public service activities have been strengthened (MOHA 2011; Vietnam 2016).

5. The system of legal documents regarding managing and utilising public employees has been updated and supplemented to better serve a modern administration. Along with the passage of laws on Cadres and Civil Servants in 2008 and Public Employees in 2010, a number of new policies aimed at enhancing the effectiveness of public employee management have been introduced. Examples include promotion via competitive examinations, selection via computer-based examinations, performance-based appraisal, talented employee retention, addressing poor performers and so on (Vietnam 2016).

Undeniably, these reform efforts have produced some positive outcomes. The time and cost for citizens and enterprises to conduct administrative procedures have been cut. For example, up until the end of 2015 , the time taken by enterprises to pay taxes on average 
reduced from 573 hours to 117 hours per year (Vietnam 2016). Citizens' satisfaction with public services has improved albeit still at a moderate level. The business environment and market economic institutions have improved. The mechanism for the operation of the market economy has been gradually improving. As a result, Vietnam has made gains in economic development and effective integration into the world's economy (Vietnam 2016; UNDP 2017). For example, Vietnam's global competitiveness increased from $64^{\text {th }}$ place in 2007 to $55^{\text {th }}$ place in 2017 out of 137 countries ranked by the World Economic Forum (Ketels, Nguyen et al. 2010; Nguyen 2017).

\subsubsection{Limitations and weaknesses}

Despite having obtained some achievements, the PAR practice still reveals several limitations and weaknesses, e.g. many goals lag behind with low effectiveness and inconsistency (Bui 2011; CPV 2016). As a result, the civil service still faces serious problems.

First, despite being regarded as the core of the PAR programmes, the performance of public employees is still far below expectations. A considerable proportion of public officials are unqualified, unmotivated, unprofessional, corrupt and bureaucratic (CPV 2012; Mau, Tham et al. 2012; CPV 2016). In one conference on public administration reform in Vietnam organised by the National Academy of Public Administration in 2011, Dr Bui The Vinh, the former director of Administration Science Institute stated that: "After 10 years, a large amount of money and time has been spent on the PARs and some things have been done. However, generally speaking, the public administration is not much different from its state 10 years ago, some aspects have even become worse" (Bui 2011).

Second, while some progress in decentralisation has been made, it still lacks consistency and does not go far enough. For example, many totally self-financing organisations continuously need the approval of higher-level agencies for personnel decisions such as recruitment, training, performance appraisal, and promotion (AcuñaAlfaro 2009). It has been said that decentralisation in Vietnam has mainly taken place in the economic management area, and has rarely occurred in the political and personnel management domain (Anh 2016). This has restrained public organisations from pursuing necessary changes in order to increase operational effectiveness.

Third, despite the reduction of central ministries, the administration machine remains cumbersome with many levels. Many state management functions and responsibilities are 
no longer compatible or necessary in the market economy but still exist e.g. state management of cultural activities and publications (Le 2011). The number of civil servants and public employees per 1000 citizens in Vietnam is currently 30.5/1000. This proportion is regarded to be relatively high compared to other countries in the regions, e.g. Indonesia (17.6/1000) and Philippines (13/1000). Consequently, salaries and fringe benefits make up to 35 percent of the state budget. This proportion is relatively large, compared to 30 percent, 13 percent and 6 percent in the Philippines, Indonesia and Japan respectively (Le 2011; Vu 2017). The cumbersome machine along with ineffective public investment projects are the principal causes for the rapid increase of public debt which reached 61 percent of GDP in 2015 (Duong 2017).

Fourth, although the reform of administrative procedures is viewed as one of the very few achievements of the PAR programmes to date, many administrative procedures remain complicated and inconvenient, particularly in "sensitive" domains such as the acquisition of land-use rights certificates, construction permits, customs procedures, and tax collection (Le 2011; Acuña-Alfaro and Tran 2016; Vietnam 2016). Several administrative procedures and regulations are still promulgated in the interest of the authorities rather than in the public interest (MOJ 2016), thereby imposing unreasonable cost burdens on citizens and enterprises (Ngo 2011).

Fifth, although several laws and decrees have been enacted, many of them have low feasibility and effect. Specifically, many laws and regulations had to be amended or abolished after only a short time of promulgation (Ketels, Nguyen et al. 2010; MOJ 2016). It has been estimated that the number of regulatory documents showing signs of violating the constitution and upper laws made up to 30 percent of the issued regulatory documents (Ngo 2016). Low-quality laws and poor policy enforcement are partially responsible for several serious problems facing the nation such as environmental pollution, deforestation, poor food safety and hygiene, transportation accidents, unemployment, low productivity, poverty and inequality. Many of these problems have been present for a long time, but have not been effectively prevented (CPV 2016; GSO 2016).

Sixth, the voice of citizens and the accountability of public organisations remain low. Vietnam's accountability index is much lower than other countries in the region, such as Thailand, Malaysia, Indonesia, and Cambodia. Citizens have very few opportunities to voice their concern or participate in the process of formulating and monitoring policies. Besides, 
corruption is still a serious problem, with Vietnam ranked at $109^{\text {th }}$ out of 137 nations (Ketels, Nguyen et al. 2010; Nguyen 2017).

Many causes for the problems above have been discussed, such as the lack of transparency and accountability of administrative agencies, the lack of resources for PAR activities, the lack of citizens' participation and the lack of determination of senior leaders to implementing changes, and so on. However, another frequently mentioned reason is the quality of civil servants who are responsible for identifying problems, making, enforcing and monitoring policies (Poon, Hung et al. 2009; Ketels, Nguyen et al. 2010; MOJ 2016; Vietnam 2016). This is consistent with President Ho Chi Minh's statement that "Civil servants are the root of all affairs... Success or failure is mostly attributed to civil servants' quality" (Nham 2013 p1). Therefore, both the party and government have identified that improving the quality and performance of civil servants and public employees is the prerequisite for building a clean and effective public administration and achieving socioeconomic development goals (Vietnam 2011; CPV 2016).

\subsection{Overview of HRM policies in the Vietnam public sector}

Although the public service has existed for two-thirds of a century, policies on managing the public service and public employees were still regarded as obsolete and incomplete. It was not until 1998 that the Ordinance on Cadres and Civil Servants was first enacted. At that time, this ordinance was regarded as the most systematic document on managing employees in the public sector. It covered many areas regarding managing cadres, civil servants and public employees, including obligations and rights; prohibitions; recruitment, training and retraining, transfers, rotation, job discontinuation and retirement.

Recently, the Ordinance was upgraded by the Law on Cadres and Civil Servants in 2008 and the Law on Public Employees in 2010. According to these laws, employees working in public organisations are classified into two categories, namely civil servants and public employees. Generally, one can understand that civil servants refer to those who are recruited and appointed to ranks (grades), posts or titles in administrative agencies at the central, provincial, district and commune levels. Meanwhile, public employees refer to those who are recruited to work in public non-business units (public service delivery institutions) such as health, research and education institutions under working contracts. However, for simplicity, the term employees hereafter implies both civil servants and public employees as the target subject of this study. In reality, policies and regulations for managing these two 
groups are not considerably different. The following are some substantial observations regarding HRM policies in the public sector.

\subsubsection{The career system}

In Vietnam, civil servants and public employees are currently managed by the career system (ISOS 2014a). Once recruited into the public sector, each employee will be placed

into one rank (grade) corresponding to their qualification regardless of the assigned job position. According to the current stipulations, there are five main ranks, including:

(1) senior specialist and equivalent;

(2) principal specialist and equivalent;

(3) specialist and equivalent;

(4) technician and equivalent;

(5) employee.

The first three ranks require the holder to have a relevant university degree. The technician rank normally requires a vocational degree (the training time from 1-2 years) while the employee rank only requires a short-term training course of less than one year (Tuyen 2013). Normally, if recruited, a university graduate will be placed into the specialist or equivalent rank. In order to be promoted to a higher rank, an employee needs to hold the current rank for at least 9 years and sit a competitive examination. Additionally, s/he has to complete a course on state management at the higher-ranking level as well as obtain acceptance from the employing organisation.

It has been said that although the career system helps public employees have a stable job in the public sector, it constrains the flexibility of organisations in employee placement and management. In fact, many senior people hold high ranks but do not have corresponding competence. This is one of the impediments to introducing employee PM, especially for goal setting and pay-for-performance because despite holding higher ranks and enjoying high salaries, many senior people can only perform simple tasks in organisations. Additionally, the career system inhibits the application of merit principles in the public service because the current regulations state that the ranks are the basis for placement, transfer, training, salary placement, and promotion. Therefore, many young and competent people miss advancement opportunities because they do not have enough seniority. 


\subsubsection{Recruitment}

Arguably, employee PM is usually affected by the recruitment practice, the following discusses how the recruitment practice has taken place and its effects on employee PM.

Before the passage of the Ordinance on Cadres and Civil Servants in 1998, staffing for public organisations was made through recommendations and referrals. The process of selection was usually secret and not transparent. Since then, competitive examinations have become the common selection method, although some work positions in mountainous areas, at borders, on islands, in remote, and extremely difficult areas can be recruited without sitting an examination. The current regulations declare that the recruitment practice must ensure the principles of publicity, transparency, competitiveness, objectivity and legality; selecting proper persons according to position specifications; and prioritising talented persons and those from ethnic minorities.

Normally, the candidates will have to sit an entrance examination of four subjects including (1) general knowledge (political system, administration apparatus, law, party policies and lines, state management), (2) specialised knowledge, (3) foreign language and (4) information technology. However, the examination subjects are relatively similar for all recruitment positions and mostly conducted under in writing. Generally speaking, the examination subjects require candidates to learn by heart rather than understand and apply the information in actual situations (Poon, Hung et al. 2009). Although the recruitment principles are clearly articulated in the laws, in reality the recruitment practice is often influenced by interpersonal relationships, nepotism, and bribes (Poon, Hung et al. 2009; Acuña-Alfaro and Tran 2016). Obviously, this not only affects the quality of cadres, but also makes PM practices such as task assignment, feedback, rewarding, discipline and so forth more difficult to operate effectively.

\subsubsection{Promotion}

Candidates for promotion must first meet general conditions and specific standards of the appointment position. General conditions include patriotism, loyalty to the revolution's ideology, integrity, anti-corruption and honesty. Meanwhile, there are specific requirements for each appointment position. For example, candidates for departmental management positions must have the advanced political reasoning certificate, a proper university degree, and the seniority of holding the principal specialist rank. Candidates for the managerial 
positions at the divisional level must have the intermediate political reasoning certificate, a proper university degree, and the seniority of holding the specialist rank.

With regard to the promotion procedure, when there is a demand or a vacancy for promotion, the head and the leadership collective (the head and deputy heads) of the agency will discuss and recommend from one to three candidates (normally, only one candidate is recommended). For the promotion practice to be transparent, democratic and open as stipulated, a meeting will then be organised with all employees for small-scale organisations (usually under 100 employees) or with key persons (managerial employees) for large-scale organisations (more than 100 employees). The purpose of the meeting is to discuss the strengths, weaknesses and compatibility of each candidate for the appointed position as well as probing their credibility through a vote of confidence by participants in the meeting. After the meeting, the agency will consult with the party committee of the agency to get the feedback and evaluation for each candidate. Based on the comments of the party committee, the leadership collective will discuss and vote to identify the most compatible candidate before issuing the appointment decision (for head or deputy head of divisions) or proposing the upper-level agency for consideration and appointment (for the head or deputy head of the agency).

Despite the seemingly sound and transparent promotion process, the promotion practice has often been influenced by nepotism, patronage and bribes (Poon, Hung et al. 2009; CPV 2018). The promotion criteria are quite abstract and easily manipulated for personal interest. With regard to this problem, the communist party of Vietnam acknowledged that "The practices of appraisal, placement and promotion are affected by interpersonal relationships, nepotism, bribery..." (CPV 2016 p.4,5,6).

\subsubsection{Salary and allowances}

As a product of the career-based system, the base salary of an employee will primarily depend on the seniority she/he holds rather than her/his performance and the value of the job (Poon, Hung et al. 2009). Each rank generally has about 6-8 salary levels. The salary level is increased every three years for the three specialist ranks, and every two years for the lower ranks. If recruited, a university graduate will be generally placed into the specialist or equivalent rank and have the same salary level regardless of their job position such as HR specialist, accountant, teacher, researcher, administrator and so forth. The base salary of a civil servant is calculated by multiplying the base salary coefficient with the minimum legal 
salary. For instance, the base salary coefficient of one new university graduate is 2.34 . The coefficient increases by 0.33 every three years if the employee is eligible (i.e. fulfils the job requirements and has no law violations).

Meanwhile, the newest minimum salary that came into effect from $1^{\text {st }}$ July 2017 is $1,300,000$ VND per month. Normally, the minimum salary is adjusted every two years based on economic growth, inflation and the status of the state budget. Accordingly, the monthly base salary of a university graduate is currently VND 3,042,000 $(2.34 * 1,300,000)$ equivalent to approximately USD 135 per month. In addition to the rank-based salary, those who hold managerial positions are entitled to a leadership allowance corresponding to their management position. For example, the leadership allowance coefficient of a divisional head is 0.6 while the leadership allowance coefficient of a departmental director is 1.00 .

Obviously, the current salary of public employees is too low compared to their counterparts in neighbouring countries and even the private sector. On average, public employees' base salary is often lower than manual workers' salaries in the private sector and make up only 50 percent of the minimum cost needed to live on (Vu 2011; Thu 2016; Hoai 2017). In 2012, the government stipulated that cadres and civil servants are entitled to a socalled public service allowance that is equal to 25 percent of the base salary. This is one of the government's efforts to achieve the goal that civil servants can live on their salaries at the average level of others in the society by 2020 (Vietnam 2011). However, to date, their current salary and allowance remain insufficient to meet the basic needs. The government's goal faces the risk of failure because its budget has to pay for a large and cumbersome staff apparatus. It is estimated that if the minimum salary increases by VND 60,000 (USD 2.6), the state budget will have to pay VND 11,000 billion (USD 500 million) more per year (Ngoc 2016).

One online survey conducted in 2012 with a sample size of 14,108 respondents showed that 77 percent believed that civil servants and public employees' salaries were not enough to cover living expenses. Of these, 35 percent responded that civil servants and public employees used their working time to do other work for extra income. Thirty percent chose to work for the public sector because this guarantees a stable job until retirement (Acuña-Alfaro and Tran 2016). The Vietnam Ministry of Home Affairs, which is mainly responsible for managing public employees, has also acknowledged that the base salary makes public servants' lives very difficult (MOHA 2011). 
In response to this problem, the Central Committee of the Communist party of Vietnam recently released Resolution No 27-NQ/TW dated 21 ${ }^{\text {st }}$ May 2018 on "reforming salary policy for cadres, civil servant, public employees, armed forces, and employees in enterprises". The solution identifies that paying the right salary (approaching market salary levels) is an investment on human resource development, which in turn enhances employee motivation and performance as well as preventing corruption. One of the principal objectives of the resolution is to increase salary so that it becomes main income and can afford employees and their families an average living standard. A part from base salary funds, the government will allocate additional salary funds for public organisations to pay for performance.

\subsubsection{Training}

In the PAR programmes, training is always identified as a key solution to enhance the quality of civil servants and public employees. In the period from 2001-2015, 10.08 million participators of cadres, civil servants and public employees were trained in state management, specialised knowledge, political reasoning, leading and managerial skills, foreign languages, information technology and so forth (MOHA 2011; Vietnam 2016). Although a large number of training courses have been delivered, civil servants and public employees are still viewed as under-qualified, particularly in the knowledge of state management and public service performing skills (Poon, Hung et al. 2009; MOHA 2011). The following are some explanations for these limitations.

First, nominations to participate in training courses often do not match the need for placement and utilization. For example, interesting and beneficial courses such as overseas training courses, and rank upgrading courses are mostly targeted at managers, senior people, and leaders' relatives rather than the people who can best benefit the organisation by attending (Trang 2011; Nho 2018).

Second, the curriculum of training programmes is often overlapping, backward and heavily theoretical (MOHA 2011; CPV 2018). For example, several subjects for training courses for the ranks of specialist, principal specialist and senior specialist, and even leadership training programmes, are almost the same. Trainees have to study some subjects such as state management, society management and defence-security repeatedly. Furthermore, the one-way teaching method is prevalent with too many people in one class. 
Thus, trainees have very few opportunities to discuss, thereby receiving knowledge passively (Trang 2011).

Third, the trainees' genuine motivation to upgrade knowledge and skills is very limited. In fact, the practices of placement and promotion in several public organisations largely rest on qualifications, degrees and certificates rather than real competence and performance (Nguyen 2011). Hence, many employees' motivation for participating in training courses is promotion and salary increases, not upgrading their knowledge and skills (Trang 2011; Mau, Tham et al. 2012).

Finally, the effectiveness of the training is substantially constrained by the absence of post-training activities such as coaching and mentoring (Poon, Hung et al. 2009). In reality, because of several causes such as the lack of specific regulations, the limited competence of supervisors and the issues regarding traditional culture, trainees have very few opportunities to apply what they have learned in their work.

The following comment sums up the training practice in this period: "Although we have obtained the achievement in terms of the number of trained people, the purpose of improving employee performance is still very far off”' (Huynh 2011, p.311).

\subsection{Current state of employee performance}

As mentioned already, one of the most important components of the PAR programmes is enhancing the quality and performance of public employees. Although many activities regarding recruitment, training and remuneration have been initiated and carried out, there is still a considerable gap between actual experience and expectation (MOHA 2011; Trang 2011; Vietnam 2016). The following sections evaluate the knowledge, skills and motivation of public employees as the primary elements comprising their performance.

\subsubsection{Knowledge and skills}

The contingent of cadres, civil servants and public employees is seen as large but not uniform in quality. The situation of both "redundancy" and "shortage" exists. Redundancy herein is associated with unmotivated and unqualified staff and the shortage refers to competent staff. Although the government has spent considerable amounts of money on training courses including short-term, long-term and even costly overseas courses, this contingent still lacks the necessary knowledge and skills such as modern public administration, laws, market economics and international integration to meet the 
requirements of the country's development (Poon, Hung et al. 2009; Mau, Tham et al. 2012; Tuyen 2013). This problem obviously has several causes, but the following are frequently cited.

First, for a long time, the selection of public employees was only conducted by assessing curricula vitae that emphasised family background, social class, morality and political quality, whereas entrance examinations only began being introduced in 1998 (Nguyen 2011). The unpublicised and uncompetitive selection in the past prevented public organisations from selecting suitable candidates. Even now, because of nepotism, personal relationships and bribery, many incompetent people continued to be taken into public agencies (Poon, Hung et al. 2009; Acuña-Alfaro and Tran 2016).

Second, the poor training quality of domestic universities is partially responsible for the problem. It has been said that because of the unreasonable and heavily theoretical curricula and obsolete training methods, graduates usually lack the knowledge and skills needed to work independently and effectively (Nguyen 2017). This is one of the principal reasons explaining why 225,500 graduates could not find jobs in 2016 while many companies could not recruit competent employees according to their need (Mai 2016).

These problems, coupled with the limitations of the training activities discussed above, have led to a considerable gap between employees' current competence and the demands of the reform process. Up to 30 percent or even 40 percent of employees in public organisations, as estimated by academics and practitioners, do not meet the job specifications (Ngo 2011; Le 2013; Vo 2015). Since 2001, the government has carried out some schemes to remove poor employees, but all these have failed to obtain the goal of downsizing civil servants and public employees by 10-5 percent (Vietnam 2016).

\subsubsection{Work motivation}

Besides limited competency, performance in the public service has suffered from the lack of employees' work motivation and public service ethics. The manifestations of these are public employees' irresponsibility, bureaucracy and apathy towards the assigned tasks and urgent needs of citizens (Nguyen 2011; Vietnam 2016). Undoubtedly, low salaries and unreasonable personnel practices, as presented above, are part of the problem. However, many people believe that the key problem stems from the working environment (Vo and Cao 2010). The prevalence of patronage, nepotism, clans, and bribery in personnel practice (Pham 2008; Diep 2011; Phuong 2012) has undermined trust, transparency and 
accountability in public agencies (Phuong 2012). Low salary and unclear development opportunity made more than 16,000 civil servants nationwide voluntarily leave government agencies from 2003 - 2007 (Poon, Hung et al. 2009; Thuy 2011). This phenomenon returned in 2017 and 2018 when some provinces such as Danang and Haugiang reported a large number of public employees, including people holding managerial positions, leaving public agencies (Nguoilaodong 2017; Doan 2018).

A survey conducted by the Institute of State and Law indicated that less than 10 percent of the top students in Vietnam's national universities chose a career in the public sector (Acuña-Alfaro 2009). This obviously raises a new challenge for public organisations to compete with the private sector in order to attract and retain talented employees (AcuñaAlfaro and Tran 2016).

In addition to the problem of limited work motivation, the public service is negatively affected by poor professional ethics. With regard to this issue, the party frankly acknowledges that "a considerable number of public officials and party members even including those who hold senior leadership and managerial positions has been affected by selfish individualism, opportunism, pragmatism, fame and trivial physical benefit" (CPV $2012 \mathrm{p} 1)$.

These problems appear to be damaging the effectiveness and efficiency of the public service. It has been said that the public service can only be comprehensively reformed if the management practice of the civil servants and public employees is radically reformed (Thuy 2011). So far, both the Communist Party of Vietnam and the government have urged its advisory institutions to review current personnel policies and develop new policies, which aim to reform the civil service and its personnel practice. Of these, reforming the appraisal practice and employee PM practice is regarded as a priority (CPV 2012; Vietnam 2012; CPV 2016; CPV 2018).

\subsection{Transiting from traditional appraisal to performance management}

\subsubsection{The appraisal practice prior to initiating the reform policy}

The appraisal practice in this period was influenced by the wars and characterised by the central planning mechanism. It was embryonic and mostly emphasised political quality and family background while overlooking competency and performance. 
The first stipulation of the appraisal practice in the Vietnamese public sector might be traced back to the early years of the formation of the nation when former President Ho Chi Minh signed Decree 76-SL dated 20 ${ }^{\text {th }}$ May 1950 on "Regulations on Public Servants". The decree provided that the heads of public organisations had authority in appraising, rewarding and disciplining subordinates. However, it did not clarify either appraisal criteria or the way to appraise (Ha, Nguyen et al. 2011).

After the victory of Dien Bien Phu in 1954, to rehabilitate the new nation, the Communist Party of Vietnam released Resolution No 18 NQ/TW dated 03 ${ }^{\text {th }}$ June 1955 on "Cadre practice". In this period, the term "cadre" was a general concept referring to all employees working for the new government, including soldiers, civil servants, public employees and workers in state-owned companies and factories. In the context of Vietnam, although the party's regulations are not legal documents, they have often dominated the society (Pham 2018). This is particularly true in the management of civil servants and public employees because the party always implements united leadership over the contingent of cadres and civil servants (Acuña-Alfaro and Tran 2016; Nguyen 2017). Moreover, the majority of cadres and civil servants, particularly those holding leadership positions, are members of the party (Pham 2018). According to the resolution, the chief purpose of the appraisal practice was for placement and promotion. The two primary appraisal criteria were morality "Duc" and competency "Tai". However, morality was often emphasised and regarded as "root". The major manifestations of morality included loyalty to the revolution and the nation, determination against imperialism and feudalism, political ideology and personality, compliance with the party's resolutions and the state's laws, and enthusiasm in performing assigned duties. In addition, the appraisal aimed to help ratees to recognise their limitations and shortcomings but had to ensure the principle of solidarity i.e. "Feedback is based on the solidarity, and feedback is to strengthen the solidarity" (Ha, Nguyen et al. 2011 p59).

Later, throughout the period of the centrally planned economy and the early years of the reform, some amendments and adjustments were made to the appraisal. However, it continued to rely on abstract criteria such as loyalty, political quality, public service ethics, discipline, morale and prestige. Further, as a result of the "full employment" policy in the subsidised and centrally planned economy, the need to improve employee performance was not a priority. Additionally, as there was no classification between different appraisee 
groups, the appraisal criteria were identical for all subjects from administrators and lecturers to workers in state-owned companies. Employee performance was often not the core appraisal criterion.

In short, it can be said that the appraisal practice, in this period, was relatively embryonic as its chief purpose was to classify employees according to loyalty, social class, and political personality for placement and promotion rather than for performance improvement. As a result, it was usually viewed as subjective, biased, prejudiced and egalitarian (Ha, Nguyen et al. 2011). Resolution No 3 of the Eighth Central Committee of the Communist Party of Vietnam in 1997 frankly acknowledged that "The appraisal practice of public officials and cadres is too subjective, unreliable, unfair, unreasonable, and lacks democracy or only artificial democracy"(CPV 1997 p2). The party urged its advisory organisations to develop a new appraisal approach that would be scientific, objective and fair.

\subsubsection{The appraisal practice from the beginning of the reform policy to the passage of the Law on Cadres and Civil Servants}

The first appraisal mandate since the time of "reform" was Decision 11/1998/QDTCCP-CCVC dated $5^{\text {th }}$ December 1998, issued by the Minister-Head of Government Commission for Organisation and Personnel (now renamed the Ministry of Home Affairs). This decision was promulgated to specify the Ordinance on Cadres and Civil Servants and regarded as the most complete legal document on the appraisal practice at that time. The decision covered the primary content of the appraisal practice, including the appraisal purpose, method, criteria and process. Accordingly, the appraisal practice was conducted to clarify competency, qualifications, performance and morality, which in turn assisted with placement, nomination, promotion, training and other personnel practices.

With regard to the appraisal process, the decision stipulated that at the end of each year, each employee had to prepare a self-evaluation based on the eight appraisal criteria below.

1) execution and compliance with laws and policies of the state

2) annual performance

3) discipline (compliance with the institution's regulations)

4) collaborative attitude with colleagues 
5) honesty in reporting and providing information to superiors

6) political personality, morality, solidarity and lifestyle

7) attitude to learn and upgrade proficiency

8) attitude of serving the people.

Annually, public organisations set aside a half or full day (depending on the scale of the units) for the appraisal practice. However, unlike western countries where appraisal meetings are usually conducted between a subordinate and his supervisor only, the appraisal practice in the Vietnamese public sector, as stipulated, was conducted by a collective meeting with all members in the division or department. In appraisal meetings, each person would present their self-evaluation prepared in advance. Afterwards, the attendees would provide feedback and judgement regarding implementing tasks, conforming to the mandates of the institution, political quality, lifestyle and so forth. At the end of the meeting, the participants rated each other on a confidential rating form (Dao 2015).

Based on the self-evaluation, comments and ratings from the collective, the head of the organisation scored each criterion with the maximum grade of 10. Based on the total points, he/she would determine the final rating decision corresponding to the four levels: (1) Excellent (equal or greater than 72 points); (2) Good (from 56 points to 72 points); (3) Moderate (40 points to 56 points); (4) Poor (less than 40 points).

\subsubsection{The current appraisal practice}

Decision 11/1998/QD-TCCP-CCVC lasted until the passage of the Law on Cadres and Civil Servants and the Law on Public Employees in 2008 and 2010 respectively. Based on these laws, the government then enacted Decree No 24/2010/ND-CP dated 15 ${ }^{\text {th }}$ March 2010 on recruiting, utilising and managing civil servants and Decree 29/2010/ND-CP dated $12^{\text {th }}$ April 2012 on recruiting, utilising and managing public employees. These decrees provided detailed guidelines on recruiting, utilising and managing civil servants and public employees, including the appraisal practice. Recently, the government exclusively enacted a single decree on the appraisal practice, namely Decree 56/2015/ND-CP dated $09^{\text {th }}$ June 2015 on appraising and rating cadres, civil servants and public employees. In general, the decree succeeded the main contents of Decision 11/1998/QD-TCCP-CCVC, albeit with some adjustments. For instance, instead of the eight criteria in the earlier document, public employees are now evaluated according to six criteria, including 
1) compliance with the policies of the Party and the laws of the State

2) political qualities, ethics, lifestyle and working style and manners

3) specialised or professional capabilities and qualifications

4) task performance progress and results

5) sense of responsibility and collaboration at work

6) attitude of serving the people.

Besides these six criteria, three more are used to appraise managerial employees, including the organisation's or unit's performance, leading and managerial capabilities and capacity to mobilise and unite employees.

Based on these criteria, employees are rated according to four levels: (1) excellent accomplishment of tasks; (2) good accomplishment of tasks; (3) accomplishment of tasks with limited capability; (4) non-accomplishment of tasks. In particular, to actualise the Laws, Decree 56/2015/ND-CP stipulates that the people rated as "accomplishment of tasks with limited capability" for two consecutive years will be transferred to other jobs while those rated as "non-accomplishment of tasks" for two consecutive years will be terminated.

The biggest achievement of these documents is creating a legal framework for the appraisal practice. Appraisal has now become a periodic and compulsory task of all civil servants and public employees (MOHA 2014a; ISOS 2014b). This result has somewhat increased accountability of employees' performance and behaviours. Second, instead of using the essay form, the new regulations stipulate the use of the appraisal criteria. This allows the essay to be combined with the scoring method, thereby somewhat discouraging the arbitrariness and increasing the convergence among raters. Third, it allows employees to openly and democratically participate in the appraisal process i.e. it allows public employees to provide comments to their colleagues and even superiors (Dao 2015). Finally, appraisal results have initially been connected to the recognition practice and other personnel practices such as succession, promotion, salary increases and discipline, albeit at a limited level (MARD 2013). Despite having gained some initial progress compared to the old appraisal system, the current appraisal practice still contains weaknesses and limitations as analysed below.

\subsubsection{Limitations of the current appraisal practice}




\subsubsection{Goal setting}

Although Decree 56/2015/ND-CP mandated that the base for appraisal is the assigned tasks or the goals set at the beginning of the appraisal year, this is rarely practised or only implemented in formalistic ways. Goal setting at the organisational level and individual level has been rather limited. Given weak accountability and weak competence of upper-level agencies, public organisations do not usually have specific goals and measurable appraisal criteria (Ha 2013). Employees are supposed to carry out routine tasks. As a result, appraisals mostly rely on what the supervisor assigned and compliance with set procedures. This not only reduces public employees' motivation and flexibility, but also makes the appraisal practice difficult. For example, competent employees might get lower appraisal scores compared to other colleagues because they are often assigned more tasks and so make more mistakes (Thuy 2011). On the other hand, for those who do not have any deliverable or outputs, the organisations cannot classify them as "non-accomplishment of tasks" because their supervisors did not assign them any (Thanh 2015). This often occurs with senior people or those who have interpersonal relationships with senior leaders, or those disliked by their supervisors.

\subsubsection{Appraisal criteria}

Despite reforms, appraisal practices still largely rely on traits and abstract criteria such as political ideology, personal morality, loyalty, honesty and lifestyle, and it is very difficult to create reliable and fair appraisal results (Ha, Nguyen et al. 2011). Currently, performance is one of six criteria making up the final evaluation score. Thus, colleagues and supervisors usually provide feedback on morality, lifestyle, personality and working attitude rather than performance (MARD 2013). Consequently, many public organisations do not fulfil assigned goals well, and even constantly get public criticism for poor service quality, but most employees and chief executives are still rated as excellent (ISOS 2014b). The defects of the appraisal criteria, coupled with the influence of traditional cultures, make most employees believe that the appraisal results do not depend on their efforts and contributions but on the subjective opinion of the appraiser (MOHA 2014a). As a result, employees only focus on conforming to regulations and avoiding conflict with others, rather than improving performance (Ha 2013).

Arguably, the unreliability of the appraisal criteria reduces work morale, as well as preventing organisations from discovering and dealing with poor performance. For several 
public organisations, the excellent rating mostly prioritises managers and senior employees. Ordinary employees will be normally rated as "good accomplishment of tasks" regardless of their performance unless they violate laws. In 2013, the Ministry of Home Affairs reported that only 0.46 percent of public officials in both local and central organisations across the whole nation were rated as "non-accomplishment of tasks" (Trung 2014). This raised a heated debate in society and the national assembly about the real number when many academics and practitioners believe approximately 30 percent of public employees are incapable of fulfilling their jobs (Ngo 2011; Le 2013; Vo 2015). One unpublished report by the Ministry of Home Affairs indicated that in 2014, the percentage of cadres and civil servants nationwide rated as "excellent accomplishment of tasks" was 36.90 percent. Other ratings were as follows: "good accomplishment of tasks": 57.22 percent; "accomplishment of tasks with limited capability": 3.73 percent and "non-accomplishment of tasks": 0.28 percent. Therefore, the people rated as "excellent" and "good" make up 94.00 percent of all public employees. The rating as "accomplishment of tasks with limited capability" mostly included those who did not attend the number of prescribed working days because of sickness, maternity leave or family affairs. Meanwhile, "non-accomplishment of tasks" is mostly applied to those who violated laws and were disciplined (Nguyen 2014). Although the current laws state that public servants rated as "non-accomplishment of tasks" for two consecutive years will be subject to consideration for termination, to date, almost no one has ever been dismissed due to poor performance (Hoi 2013).

In this regard, Dr Nghiem Van Loi, a lecturer at the University of Labour and Social Affairs, argues that this paradox derives from the inappropriateness of appraisal criteria that are developed to help public servants to be rated as "excellent" or "good" but do not contribute to the accomplishment of the mission and tasks ( $\mathrm{Vu} 2014$ ).

\subsubsection{Feedback}

As regulated, feedback is still undertaken through collective meetings. This method seems to be transparent and democratic because it allows employees to provide comments about colleagues and even superiors. However, in fact, it is often ritualistic, inaccurate and ineffective (ISOS 2014a). In addition to the appraisal method, these shortcomings are likely come from cultural values, such as Confucianism, which emphasises interpersonal relationships, collectivism, seniority preference and harmony in social relationships (Zhu, Warner et al. 2007; Warner 2010). 
Like other personnel practices, PA is regularly affected by personal relationships, power distance, face-saving and high collectivism (Stanton and Pham 2014). The mindset, "I am easy for you so you in return will be easy for me" is very common in appraisal meetings (Ha 2013 p.3). Therefore, instead of attempting to improve performance, many employees only focus on building and consolidating interpersonal relationships with colleagues and supervisors (ISOS 2014a). Co-workers, even chief executives, tend to avoid providing frank comments because of the fear of displeasing others (MARD 2013). The culture of face-saving makes people feel very uncomfortable when receiving negative comments in front of a crowd. They tend to assume that the person providing negative comments is a rival. Therefore, appraisal meetings are like an event where people praise each other rather than providing honest feedback for improvement.

Once rated as "excellent" or "good" by the collective, the chief executive rarely adjusts these to lower levels in order to avoid conflict with the subordinates. As a result, many organisations report that the percentage of employees rated as "excellent" is more than 70 percent, even with some more than 90 percent (Ha 2013).

\subsubsection{Frequency of appraisal}

Like any traditional appraisal system, the current appraisal practice takes place once a year. Therefore, employees are often not aware of their shortcomings and weaknesses, nor are they corrected in a timely manner. Prior to annual appraisal meetings, employees prepare a self-evaluation that mainly lists accomplished tasks without considering quality. Weaknesses and limitations are often overlooked or purposely ignored. Meanwhile, because detailed notes are not taken, the supervisors can only provide feedback to the employees in a perfunctory way (Ha, Nguyen et al. 2011). As a result, the weaknesses and limitations of the preceding year are most likely to be repeated because they are not included in the action plans for the following year. Additionally, for some people, annual appraisal meetings are probably viewed as a good opportunity for personal retaliation. The annual appraisals sometimes creates conflict and depression rather than helping employees to realise their weaknesses (Nguyen 2015). Consequently, the infrequency of appraisal, coupled with collective feedback and poor goal setting, largely constrains the goal of improving employee performance (Ha, Nguyen et al. 2011). 


\subsubsection{Connection to rewards and other personnel practices}

Another frequently mentioned limitation in the current appraisal system is the limited connection between the appraisal result and other personnel practices (Thuy 2011; MARD 2013). This is very common among public organisations, though the legal documents clearly state that appraisal results need to be used as a basis for placement, appointment, training, retraining, rewarding and discipline. There are various reasons for this problem. First, given abstract criteria, the appraisal results are often unreliable and invalid, so the agencies are very reluctant to connect appraisal results with administrative decisions, including pay. Second, there is a lack of specific guidance from the government to connect appraisal results to rewards effectively. Third, personnel practices are usually influenced by nepotism, patronage, interpersonal relationships and even bribery (Hoang 2014; Acuña-Alfaro and Tran 2016; CPV 2018). Consequently, the limited connection between performance and rewards has steadily undermined employees' motivation and work morale, especially young and competent employees because they do not see their future career development opportunities. Arguably, many capacity building programmes, including costly overseas training activities cannot maximise their potential because of the absence of practices based on merit principles (Ketels, Nguyen et al. 2010).

In short, the aforementioned shortcomings together make the performance appraisal practice ritualistic and ineffective. In one official document circulated to ministries and provinces in 2014, the Ministry of Home Affairs warned that "the appraisal and rating results of ministries and localities do not sufficiently and accurately reflect the actual performance, responsibility and discipline of civil servants and public employees" (MOHA 2014b p1). Consistent with this message, The United Nations Development Programme (UNDP) in Vietnam commented: "At the moment, the evaluation of civil servant performance is only a formality. The method and criteria for evaluation are inappropriate with no emphasis on the performance result and efficiency... the civil servant evaluation system fails to assist the government to understand the working competency of each civil servant in order to use the right person in the right place..." (Poon, Hung et al. 2009, p.9).

Aware of the limitations of the current appraisal system, in Decision No. 1557/QDTTg dated 18 October 2012 for approving the scheme to speed up reform of the public service and civil servants, the Prime Minister emphasised: "Continue to innovate the appraisal practice for cadres and civil servants. Accordingly, appraisal needs to be based on 
employee performance and effectiveness...the managers need to pay more attention to outputs, achievement, and contribution and regard these elements as chief criteria in appraising quality, competence and qualification of cadres and civil servants..."

\subsubsection{Innovation towards performance management}

\subsubsection{Antecedents for innovation towards performance management}

Although the majority of public agencies are still adhering to traditional appraisal, some public organisations have proactively sought better alternatives in managing their employees' performance. The following will discuss some antecedents promoting the innovation process.

First, under competitive pressure to attract investment projects, particularly foreign direct investment (FDI) projects, some provinces are trying to improve their business environment. This process was accelerated when the Vietnamese Chamber of Commerce and Industry (VCCI) recently publicised the yearly Provincial Competitiveness Index (PCI). Accordingly, VCCI ranks provinces in terms of governance quality, business environment, transparency, public administration reform and so forth. As a result, many provinces have had to pay attention to improving the quality of the public service. For instance, the Provincial People's Committee (PPC) of Bacgiang has piloted a scheme evaluating chief executives as a measure to increase the accountability and performance of public organisations. Accordingly, the chairman of the PPC evaluates the performance of chief executives based on the agency's performance of five core tasks. Each core task is embedded with measurable appraisal criteria (BacGiang 2013). Meanwhile, the PPC of Danang city, with the approval of central agencies, has introduced an output-based appraisal scheme for all its departments and districts. Such programs are often called 'fence-breaking' because they allow agencies to experiment with new policies outside the scope of current regulations. The purpose of the innovation is to increase the reliability of appraisal results aimed at classifying employees into different groups in terms of performance and competence in order to make proper personnel decisions (Danang 2014). In addition to Bacgiang and Danang, some other provinces such as Laocai, Quangning, Longan and Hanoi have been piloting appraisal schemes aiming to improve employees' responsibility and motivation, and the effectiveness of other personnel practices such as training, recognition, reward and promotion (Dao 2015). 
Second, to enhance the operational effectiveness of public service delivery institutions (public non-business units), the government has enacted some decrees on self-financing mechanisms. Examples include: Decree No. 115/2005/ND-CP dated December 05, 2005 on the mechanism of autonomy and self-management for the tasks, finance and assets, organisational structures and payrolls of public scientific and technological organisations; and Decree No. 43/2006/ND-CP dated April 25, 2006 on providing for the right to autonomy and self-responsibility for task performance, organisational apparatus, payroll and finance of public non-business units. The primary objective of these decrees is to "socialise" public services by transferring the operational mode of public service delivery organisations from totally subsidised organisations to business-like organisations. As a result, these institutions will have to compete to provide public services and find external contracts in order to generate revenue.

Therefore, for their survival and development, public service delivery organisations will have to enhance self-competitiveness by improving the quality of services and reducing production and management costs. Consequently, they start refining performance standards to make employee performance align with organisational goals. Besides, they try to reward for perforamance to motivate and retain good performers, while addressing poor performers more seriously.

Additionally, to accelerate the operational effectiveness of self-financing organisations, the state has stipulated that public service delivery institutions will be granted autonomy, including HR autonomy corresponding to the extent to which they can finance their own operational and investment costs. For example, for totally self-financing organisations, the chief executives have authority in recruitment and selection, placement, nomination of the head of units, transfer, discipline and termination; in particular, they can pay employees supplementary salaries up to two times the base salary.

Along with increased autonomy and accountability, the innovation process of employee PM has been supported by some other important antecedents. The first is the role of chief executives in the organisations initiating PM schemes. While the central planning mechanism has made many public managers passive and inert, there are chief executives who have vision, public interest and courage to pursue reform initiatives. These chief executives are willing to take risks to adopt new management approaches that may not yet be compatible with current regulations. The second is the assistance of international 
organisations in terms of financial resources and technical consultancy. For example, in 2012, Danang city was aided by UNDP to carry out PAR, including innovating appraisal criteria, developing an online feedback system and connecting performance with pay (Danang 2014). Additionally, the international integration process has enabled Vietnam to approach advanced management methods, including employee PM. For example, thanks to sending staff to study abroad, referring to management experiences of foreign direct investment (FDI) enterprises, or even searching on the internet, public organisations can proactively perceive theories and principles in designing and implementing employee PM systems.

\subsubsection{Characteristics of the innovation of performance management practice}

Although the antecedents discussed above might not be strong enough to lead to a comprehensive change in employee PM practice, they initially created necessary conditions for innovation in the public sector, particularly for public service delivery organisations and local agencies with greater autonomy. The initial information collected indicates that there is relative diversity in terms of the appraisal method, criteria and process, as well as the extent to which performance is connected to rewards and other personnel practices. Nevertheless, in general, these schemes share some common characteristics, including:

1) better integrating individual goals with organisational goals

2) emphasising output-based appraisal

3) quantifying appraisal criteria

4) linking performance with rewards and sanctions

5) encouraging the participation of employees in the innovation process of PM

6) addressing poor performers.

As a result, the schemes have engendered promising outcomes such as improving accuracy and fairness in appraisal, improving work responsibility and enhancing employee motivation (BacGiang 2013; Danang 2014). However, it is worth noting that these outcomes are not yet sustainable because of the lack of uniform and stable policies from the government, weak accountability, incompatibility of traditional culture, the departure of initiators in pilot agencies and so on. 


\subsection{Summary of Chapter 2}

Irrespective of the efforts in implementing PAR programmes for approximately three decades, the public service in Vietnam still faces serious challenges. One of the most important causes is the subject of the public service i.e. the contingent of public employees. Currently, a large proportion of employees in the public sector remain incompetent, unmotivated, irresponsible and bureaucratic. It has been said that these limitations derive from historical legacies with hundreds of years under a mandarin structure of society rule, and tens of years under the bureaucratic mechanism of "beg and give" and administrative orders. As a result, the public service has become too cumbersome with a large number of unqualified employees while lacking competent and motivated people. Improving employee performance has become one of the priority tasks of the government aimed at developing an effective and efficient public service.

At present, employee PM is still traditionally implemented and usually affected by detrimental factors such as obsolete personnel policies, weak accountability, egalitarianism, nepotism, interpersonal relationships and corruption. In fact, the majority of public organisations still adhere to the traditional appraisal practice that is characterised by annual appraisals, abstract criteria, collective feedback and weak links with rewards and other personnel practices. These cause the PM practice to be ritualistic and less fruitful.

Fortunately, the efforts of the PAR process over the last few decades have produced the initial antecedents for renovating employee PM. Examples include provincial competitiveness rankings, increases in performance accountability and autonomy, senior managers with entrepreneurial leadership and so on. While the PM reform efforts are not yet mainstream, they have created antecedents necessary for innovating PM practice. Together, these characteristics produce a context suitable to examine whether employee PM innovation can improve employee attitudes and organisational outcomes. Simultaneously, this context is very compatible with investigating enablers and barriers to the innovation process of PM practice in a transition economy. The next chapter reports on the literature review of employee PM in transitional countries. 


\section{CHAPTER 3. LITERATURE REVIEW}

This chapter reviews the literature regarding implementing employee PM in the context of transitional countries. Based on this review, the research questions and hypotheses for the research project are developed. The chapter begins by briefly presenting the need to carry out Public Administration Reform (PAR) in the public sector in transitional countries. Then it discusses the birth and the definition of employee PM. This is followed by reporting trends in adopting and implementing employee PM in transitional and developing countries. Next, it presents theories and arguments regarding developing: (i) Hypothesis 1 about the relationships between employee PM and desirable outcomes; (ii) Hypothesis 2 and 3 about the influence of institutional and capacity factors on the development of employee PM; and (iii) Hypothesis 4 and 5 about the moderation effect of organisational cultures and intervention practices. Finally, based on these hypotheses, it proposes a conceptual framework before moving to the conclusion of the chapter.

\subsection{Public administration reform in transition economies}

The term "transition economies" refers to countries experiencing a transition from a centrally planned to a market economy. According to this definition, transition economies include 32 countries in Europe, Central Asia and East Asia. They include Albania, Armenia, Azerbaijan, Belarus, Bosnia and Herzegovina, Bulgaria, Croatia, Czech Republic, Georgia, Estonia, Hungary, Kazakhstan, Kyrgyz Republic, Latvia, Lithuania, Macedonia, Moldova, Romania, Poland, Russia, Serbia and Montenegro, Slovak Republic, Slovenia, Tajikistan, Turkmenistan, Ukraine, and Uzbekistan, Cambodia, China, Laos, Mongolia and Vietnam (Suhomlinova 2006).

For almost all these countries, PAR is viewed as an indispensable activity with the aim of facilitating the transition process (Tong, Straussman et al. 1999; Painter 2003; RandmaLiiv 2005; Damiran and Pratt 2008; Veselinović 2014). Arguably, the legacies from centrally planned economies have left enduring negative consequences for the contemporary administration such as bureaucratic and inert administration apparatus, discretionary governance, poor accountability and transparency, weak work ethics, poor performance and pervasive corruption (Poon 2004; Damiran and Pratt 2008; Meyer-Sahling 2009; Veselinović 2014). Public administration reform (PAR) has been highlighted as one of the imperative conditions for creating successful and sustainable economic development 
(Veselinovic 2014). Indeed, the effectiveness of the public sector positively relates to developmental goals such as GDP per capita, adult literacy and reduced infant mortality (Kaufmann, Kraay et al. 1999).

In each country, depending on conditions and development levels, PAR can be designed in different forms to meet different goals. For example, PAR in Serbia focuses on the restructuring of state-owned enterprises, the pension system, the health care system and the social protection system (Veselinović 2014). PAR in Western Balkan countries centres on the requirements for joining the European Union i.e. the basic EU legal principles for candidate countries, including trust and predictability, openness and transparency, accountability, efficiency and effectiveness (Cierco 2013). PAR in Kazakhstan targets decentralisation, civil service reform, e-governance and the promotion of the role of civil society in order to enhance government effectiveness, control corruption and reduce poverty (Bhuiyan and Amagoh 2011). PAR in China centres on restructuring state-owned enterprises and service delivery units, downsizing the government and reducing the number of public employees, and reforming the personnel management system. The Chinese government is also interested in simplifying administration procedures by establishing one-stop shop service centres and transforming government's role from micro-management to macroregulation (Ngok and Zhu 2007; Christensen, Lisheng et al. 2008). In Vietnam, PAR focuses on refining the constitution, rationalising the organisation of the state administrative apparatus, improving the quality of civil servants and public employees, reforming public finance and simplifying administration procedures.

Regardless of the rhetoric and enormous support from international donors, many empirical studies have reported that PAR in transitional countries only achieves limited success. This problem has been attributed to a wide range of causes. Examples include:

1) stagnation of political reform (Ngok and Zhu 2007);

2) incompatibility of the current administration culture (Christensen, Lisheng et al. 2008);

3) lack of participation from citizens in the reform process (Damiran and Pratt 2008);

4) poor accountability and pervasive corruption (Jacobs 2004; Moon and Hwang 2013); 
5) lingering legacy of the Soviet-style command economy, old administrative habits, political interference, weak state institutions and disrespect for rules (Cierco 2013).

However, one of the most frequently discussed reasons is the poor performance of public employees who directly participate in the process of developing, implementing and monitoring the reform programmes (Jacobs 2004; Berman, Bowman et al. 2015; Ho and Im 2015). Ingraham and Kneedler (2000) claim that since government activities are highly personnel-intensive, managing public employee performance is central to enhancing the quality of the public service. Therefore, the way in which their performance is managed determines the operational effectiveness of organisations in fulfilling their goals (Manasa and Reddy 2009). While instances of exemplary performance exist, irresponsibility, corruption, poor performance and low work ethic are particularly widespread and harmful in transitional and developing countries (Jacobs 2004; Damiran and Pratt 2008; Cierco 2013). As a result, improving accountability, motivation, work ethics and proficiency of public employees has been highlighted as an essential condition for reform initiatives to be fruitful (Tong, Straussman et al. 1999; Jacobs 2004; Moon and Hwang 2013).

Recognising the shortcomings of public employees, past employee performance reforms have targeted selection, appraisal and discipline, and have provided some changes to performance rewards. However, agreement exists that the above employee problems persist (Tong, Straussman et al. 1999; Randma-Liiv 2005; Poon, Hung et al. 2009; Berman 2015). Indeed, improving employee performance has proven difficult in developing and developed countries (Jacobs 2004; Holloway, de Waal et al. 2009; Burns and Zhiren 2010).

\subsection{Evolution and definition of employee performance management}

\subsubsection{Evolution of employee performance management}

Before discussing the concept of employee PM, it is worth noting that employee PM is the evolution of performance appraisal (Bretz Jr, Milkovich et al. 1992; Kinicki, Jacobson et al. 2013) though in the literature the two concepts are often used interchangeably (Law 2007). While performance appraisal has been used for centuries, employee PM has only been used in the last few decades (Pulakos and O'Leary 2011; Seniwoliba 2014).

Performance appraisal (PA) is generally defined as a process of evaluating an individual's behaviour and accomplishments in the past and present to differentiate between members in the organisation. The primary purpose of PA is to arrive at objective administrative decisions such as selection, promotion, transfer, remuneration, dismissal and 
so on (Heyel 1958; DeVries, Morrison et al. 1981; Bratton and Gold 2012). In many transitional countries, PA is mostly used for discipline purposes rather than developmental and incentive purposes (Meyer-Sahling 2012).

In a traditional appraisal system, as one feature of transitional countries, employees are often appraised by behaviours and abstract, non-performance related criteria (RandmaLiiv 2005; Dzimbiri 2008; Poon, Hung et al. 2009). Hence, traditional PA continues to encounter persistent defects such as cognitive issues, leniency and central errors, and other biases such as the political motivation of raters (Bretz Jr, Milkovich et al. 1992; Longenecker and Gioia 2001; Merritt 2007; Gardner 2008). Indeed, it has been said that PA may create more problems than it solves (Thomas and Bretz Jr 1994).

The negative impacts of a poor appraisal system have been widely discussed in the literature. First, performance appraisal is likely to undermine job satisfaction and organisational commitment, thereby increasing the probability of decreased productivity and employee turnover (Mustapha and Daud 2012; Rubel and Kee 2015). This is because employees usually evaluate themselves more favourably than others do (Murphy and Cleveland 1995 p.337) and employees usually perceive performance appraisal as a threatening and punitive tool. Second, an ineffective appraisal system not only fails to improve communication but also exacerbates tension and conflict between supervisors and employees (Pettijohn, Pettijohn et al. 2001). Appraisal systems often force raters to make distinctions that are simply not realistic or functional. Thus, subjective evaluations are unavoidable. Employees often perceive that ratings are in inaccurate and unfair (Hulin 1982; Murphy and Balzer 1989; Ilgen, Barnes-Farrell et al. 1993). This can even be exacerbated if employees perceive that appraisal outcomes stem from the personal motivation of raters (Wiese and Buckley 1998; Bawole, Hossain et al. 2013). Third, individual PAs might undermine the morale of work groups because such a system tries to apportion credit or blame to individual members of a team (Roberts 2003; Law 2007). This can result in interemployee jealousy, hostility and competition, thereby preventing the cooperation of members in the future (Law 2007).

The failure of traditional employee management practices has led to the birth of employee PM (Bretz Jr, Milkovich et al. 1992; Armstrong and Baron 2005; Kinicki, Jacobson et al. 2013). There is a perception that the methods of appraisal are important, but they may not be the most important determinants of an effective system and there are calls 
to pay more attention to broader and more effective PM practices and contextual variables (Murphy and Cleveland 1995; Ohemeng 2009; Haines III and St-Onge 2012).

Although employee PM was first used in the 1970s (Patten 1977; Pulakos and O'Leary 2011), it has been quickly accepted and adopted in the private, then public sectors in many countries (Ohemeng 2009). Employee PM has been promoted by reformers advocating the doctrine of New Public Management (NPM) (Vallance 1999; Decramer, Smolders et al. 2012). This doctrine underscores characteristics such as "stress on private sector styles of management practices", "explicit standards and measures of performance", "greater emphasis on output controls", "shift to greater competition in the public sector" and "stress on greater discipline and parsimony in resource use" (Hood 1991 p 4,5).

To improve employee performance, employee PM emphasises (i) goal-based feedback and appraisal, (ii) coaching, (iii) consequences for performance (rewards and punishment), and (iv) employee participation in decision-making (Luecke and Hall 2006; Aguinis 2009; Ohemeng 2009; Cho and Lee 2011; Kinicki, Jacobson et al. 2013). Employee PM has been used in support of various organisational and reform objectives such as improved public service delivery, increased efficiency, improved transparency and upgraded organisational capacities such as innovation (Ohemeng 2009). It is also said that employee PM is an effective tool to help governments at all levels to do more with less (Mani 2002). For many reformers, employee PM has become one of the most advocated techniques to create a professional and accountable administration (Cardona 2006; Ohemeng 2009). Apart from the above benefits, it can effectively address many critical issues in developing countries such as accountability (Bouckaert and Halligan 2007), corruption (Goh 2012; Ciobanu and Ristea 2015) and incompatible habits (Moriarty and Kennedy 2002).

\subsubsection{Definition of employee performance management}

In the literature, the concept of performance management is often used with at least three different meanings. First, it is regarded as a system to manage organisational performance. Second, it is used to manage employee performance. Third, it is used to manage both organisational and employee performance (Williams 1998). In this thesis, performance management focuses on employee performance management.

To date, there are various definitions of employee PM in the literature. For example, Heathfield (2007) defines employee PM as "the process of creating a work environment or setting in which people are enabled to perform to the best of their ability" (p.8). A more 
recent definition is that of Kinicki, Jacobson et al. (2013) who define that "performance management is a process consisting of managerial behaviours aimed at defining, measuring, motivating, and developing the desired performance of employees" (p.4). However, this research uses the definition of Aguinis (2009) because it is well-suited for the purpose of this research and frequently cited in the literature. Accordingly, employee PM is defined as "a continuous process of identifying, measuring and developing the performance of individuals and teams and aligning performance with the strategic goals of the organisation" (p.2)

While several employee PM models have been proposed so far, there is "no articulated consensus on what the main components of PM are" (Schleicher, Baumann et al. 2018, p.2211). As a broad definition, employee PM can include all HR practices used to give employees the means, work motivation, and opportunities to enhance organisational performance (DeNisi and Smith 2014). Nevertheless, the process of employee PM usually involves setting goals, monitoring and appraising performance, providing feedback, coaching, training for development, encouraging participation, providing rewards for good performers and handling poor performers (Lawler 2003; Luecke and Hall 2006; Aguinis 2009; Pulakos and O'Leary 2011; Tung, Baird et al. 2011; Haines III and St-Onge 2012; Kinicki, Jacobson et al. 2013; OPM 2017). The overall objective of an employee PM system is to promote desirable behaviours and increase individual performance, which in turn improves organisational performance (Armstrong 2006; DeNisi and Pritchard 2006).

Compared to traditional PA, PM has some fundamental differences. The most notable is perhaps the ultimate purpose. While the primary purpose of PA is to provide information for administrative decisions, employee PM aims to improve employee and organisational performance in the future (Lebas 1995; Armstrong and Baron 1998; Aguinis 2009). However, it is noteworthy that performance appraisal is still a substantial part of the performance management system that consists of interconnected practices (Lebas 1995; Beardwell and Claydon 2007; DeNisi and Murphy 2017).

\subsection{Introduction and challenges of employee performance management}

\subsubsection{Employee performance management in the public sector}

For the last twenty years, many OECD member countries have implemented PM systems as a management tool to increase quality and efficiency in public service delivery (Lah and Perry 2008; Kong, Kim et al. 2013). As a new reform trend, many leaders of 
developing countries have also been eager to adopt employee PM techniques with the aim of improving the effectiveness of people management. The following examples provide some information regarding the official embrace of employee PM in developing countries.

In 1992, the Malaysian government initiated a new performance appraisal system with the components of performance planning, performance monitoring, provision for feedback, development of action plans to coach and counsel employees, and performance-based rewards. The overarching purpose of this scheme was to improve individual performance and then public sector performance (Shafie 1996).

In 2001, the Estonian government introduced a pay-for-performance scheme with the purposes of (i) better linking between individual performance and organisational goals; (ii) improving teamwork; (iii) increasing public sector transparency; (iv) increasing the effectiveness and efficiency of public agencies (Randma-Liiv 2005).

In an effort to solve the Asian financial crisis in 1997 and strengthen competitiveness, the Korean government introduced a performance management system on two levels i.e. organisational and employee performance management. The employee PM system consisted of four components. The first was a performance agreement based on the organisation's strategic goals that were accompanied by performance indicators, measurement methods, performance targets and accomplishment plans. The second was used to gauge employees' performance and provide feedback for improvement. The third was a 360-degree evaluation as a supplementary appraisal tool to constrain the problems deriving from one evaluator such as leniency, personal bias or subjectivity. The fourth was pay-for-performance aiming to encourage competition and improve performance (Kong, Kim et al. 2013).

Further, the literature has reported the innovation of employee PM in the public sector in several other developing countries. These include China (Burns and Zhiren 2010; Liu and Dong 2012); Ghana (Ohemeng, Zakari et al. 2015); Maldives (Asim 2001); Thailand (Koonmee 2009); Southeast European transitional countries (Jankulović and Škorić 2013); Uganda (Nambi Karuhanga and Werner 2013); Botswana (Dzimbiri 2008); Kenya (Obong'o 2009); and Vietnam (Danang 2014; Dao 2015).

\subsubsection{Challenges of employee performance management in developing countries}

Theoretically, employee PM can benefit employees, organisations and the public interest because it can improve the direction of behaviour, the level of motivation and the 
proficiency of employees (De Waal and Gerritsen-Medema 2006; DeNisi and Sonesh 2011). However, it is worth noting that the successful implementation of employee PM is still a global challenge even in the most developed countries where the failure rate has been estimated approximately 60 percent (Holloway, de Waal et al. 2009; Haines III and St-Onge 2012). Arguably, employee PM is a sophisticated activity to master and engender desired outcomes (De Waal 2007). Many studies have shown that successful PM projects are rather limited (Ochoti, Maronga et al. 2012; Seniwoliba 2014). Moreover, research reveals that up to 80-90 percent of HR professionals perceive that their employee PM systems are not effective in improving organisational performance (Haines III and St-Onge 2012). Meanwhile, less than one third of employees think that the employee PM system can help them to increase performance (Pulakos 2009). In reality, employees and supervisors often dislike employee PM and see it as a control mechanism or an unnecessary administrative burden (Thomas and Bretz Jr 1994; Biron, Farndale et al. 2011; Kim 2014).

This failure can stem from various causes. One widely acknowledged reason is the lack of robust empirical research. Posthuma and Campion (2008) comment that despite a lot of attention paid to the design of an employee PM system, very few studies are done on how it really functions when adopted. Likewise, Pulakos and O'Leary (2011) posit that the ability to understand the most important elements of employee PM can help to consolidate its overall effectiveness. However, the literature lacks empirical research to establish the real impact of PM practices recommended in theory on the desirable outcomes such as employee attitude and performance (Tung, Baird et al. 2011; Haines III and St-Onge 2012). To date, regardless of a large amount of research devoted to employee PM, this practice is still regarded as the "Achilles Heel" of human resource management (Pulakos 2009) and "the formula for effective performance management remains elusive" (Pulakos and O'Leary 2011 p.147). Employee PM is still a controversial topic with little consensus on how it can be improved (Vallance 1999; Magee 2002; Ohemeng 2009). Indeed, employee PM "has been one of the most praised, criticised, and debated management practices for decades" (Lawler 1994, p.16)

Apart from the challenges above, the adoption of employee PM in developing and transitional countries is even more difficult. In fact, the majority of employee PM schemes in developing countries are not effective in achieving desirable outcomes (Shafie 1996; Asim 2001; Randma-Liiv 2005; Liu and Dong 2012; Ohemeng, Zakari et al. 2015). There 
are sceptics about the applicability of PM in developing countries because the western context where it was first innovated and evolved differs from developing countries economically, culturally, legally and politically (Vallance 1999; De Waal 2007; Ho and Im 2015). In fact, there are extreme perspectives that employee PM is not suitable for developing countries (Mendonca and Kanungo 1996; Schick 1998; Verheijen and Dobrolyubova 2007). In this regard, scholars generally agree that reinventing PM systems is most likely to be unfruitful or cause unwanted results if contextual factors are not taken into account (Mendonca and Kanungo 1996; Wood and Marshall 2008; Pulakos and O'Leary 2011). All these reasons have led to an urgent need to study the use and implementation of employee PM in non-western settings (Vallance 1999; Fletcher 2001; Siaguru 2011).

\subsection{Employee performance management practices in transitional countries}

As presented earlier, employee PM relates to a wide range of HR activities used to motivate and increase employee performance. Prior studies show that this reform may fail when, for example, appraisal is separated from consequences (Liu and Dong 2012); when pay-for-performance is not based on objective performance standards (Randma-Liiv 2005) and when feedback lacks employee input and participation (Chiang and Birtch 2010). Hence, a combined approach is increasingly used, e.g. (Gorman, Meriac et al. 2017)

In reality, the combination of specific employee PM practices can vary from organisation to organisation. Based on the literature review and the research context, this research examines the use of five employee PM practices, including (i) goal-based appraisal; (ii) feedback; (iii) reward-for-performance; (iv) addressing poor performers; and (v) encouraging employee participation. The choice of these five practices derived from analysis of several books and articles on employee PM e.g. Shields (2007), Aguinis (2009), Ohemeng (2009), Fletcher and Williams (1996), Lawler (2003), Roberts (2003), Luecke and Hall (2006) as well as studies of PM innovation in developing countries, e.g. Shafie (1996), Randma-Liiv (2005) and Burns and Xiaoqi (2010).

Although coaching and development training are often discussed in the literature in western countries, they are not common in developing contexts, particularly in China and Vietnam (Poon, Hung et al. 2009; Liu and Dong 2012). Early interviews in the research revealed that supervisors did not usually have the knowledge and skills to coach their subordinates, and supervisors did not often want to coach and train the subordinates because they were afraid that the subordinates would become more competent and compete with 
their positions in the future. To date, the government has not had any specific instruction for this practice. As a result, coaching was not included in this research.

The following discusses the content of each of the five PM practices, as well as how the developing context influences the implementation of each practice.

\subsubsection{Goal-based appraisal}

Goal-based appraisal is at the heart of the PM system (Lacho, Stearns et al. 1991; Bititci, Carrie et al. 1997; Kinicki, Jacobson et al. 2013). It is understood as a process in which an employee's performance is measured by specified goals set at the beginning of the performance period (Vallance 1999). Setting measurable job goals that correspond to organisational goals provides a framework to assess and support performance. Goal-based appraisal not only enables employees to utilise their resources in more creative and optimal ways but also holds employees accountable for their performance. Compared to traditional appraisals that rely on abstract criteria, goal-based appraisal is more objective, fairer and easier for providing feedback and rating. In a Delphi study, Davis (2015) finds that setting and appraising goals based on SMART (Specific, Measurable, Appropriate, Realistic and Time-bound) are the most important practices of an effective employee PM system. Arguably, goal-based appraisals have positive associations with employee motivation, job satisfaction, and individual and organisational performance (Roberts 2003; Latham, Borgogni et al. 2008).

Although public organisations globally have difficulty in setting goals, transitional countries face particular challenges (Rainey, Backoff et al. 1976; Montoya and Graham 2008). For example, weak accountability and frequently changing priorities, as a feature of the transition process, can make organisational goals particularly ambiguous and unstable (Randma-Liiv 2005; Liu and Dong 2012). Egalitarianism, collectivism and process-oriented cultures lead to weak or unchallenged goals, or the valuing of political loyalty and personal qualities instead (Poon, Hung et al. 2009; Liu and Dong 2012; Seniwoliba 2014; Stanton and Pham 2014). While the primary appraisal criteria in western countries emphasise efficiency and outcomes, those of ex-communist countries rely on the manner or the process (Koubek and Brewster 1995). The lack of goal-based appraisals leads to unfair and inaccurate ratings, which in turn impedes providing constructive feedback, rewarding good performers and handling poor performers. Arguably, this is one of the most frequent 
rationales for the failure of PM systems in transitional and developing countries (Tong, Straussman et al. 1999; Poon, Hung et al. 2009; Denkyira 2014).

Notwithstanding the unfavourable conditions in the developing context, goal-based appraisals should be viewed as an important part of PAR in general and PM in particular. This is because it helps to connect employee effort with organisational goals and creates a solid base for sound personnel practices. To overcome these constraints, some countries such as Malaysia, Korea and Thailand stipulate that the employee PM cycle must begin by establishing organisational goals that will be monitored and evaluated by superior authorities with specific criteria. The organisational goals are then cascaded to departmental and individual levels (Shafie 1996; Koonmee 2009; Kong, Kim et al. 2013). Similarly, the Vietnamese government has recently stipulated that individual appraisals need to be based on the tasks assigned at the beginning of the performance period. The extent to which these goals are fulfilled must be used as the major criteria for feedback and rating (Vietnam 2015). Additionally, under the pressure of having to self-finance, as discussed in the previous chapter, some public organisations in Vietnam are trying to connect individual goals with organisational goals to increase the organisation's effectiveness.

\subsubsection{Ongoing feedback}

Ashford and Cummings (1983) refer to feedback as information that signifies how well individuals are meeting set goals. Accordingly, feedback involves the perception and evaluation of relevant stakeholders such as supervisors, peers, subordinates or customers about the appraisees' behaviours and performance. The feedback process clarifies contributions to organisational and broader purposes, supports task performance, motivates good performers, and helps poor performers improve (Somerick 1993).

Unlike traditional performance appraisals (PA) that only focused on annual assessments of past behaviour, employee PM is based on the premise that employees should receive timely, constructive and frequent feedback about their current performance to enhance future success (Kinicki, Jacobson et al. 2013). Timely and constructive feedback is associated with increased satisfaction, motivation and performance (Lawler and McDermott 2003). Clampitt and Downs (1993) found that feedback has a close correlation with employee productivity because it has a strong effect on employee morale and motivation. Risher (2011) even asserts that "individuals cannot improve their performance unless they receive some form of feedback" ( p.274). 
Despite being useful in theory, for several developing countries, particularly those influenced by Confucianism, cultures of harmony and face-saving make honest and direct feedback particularly difficult (Hofstede 1998; Vallance 1999; Chiang and Birtch 2010). Feedback is often avoided, softened, or given in an indirect and subtle manner (Larson Jr 1989; Aycan 2005). In these countries, feedback is also constrained by a traditional appraisal that relies on job-irrelevant criteria and infrequent appraisal, untrained managers and high power distance (Randma-Liiv 2005; Siaguru 2011; Liu and Dong 2012).

Although these contextual factors should not be overlooked, it does not mean that feedback practice is not able to be improved. In such contexts, the feedback practice is still likely to be fruitful if the designers pay attention to these factors in the process of developing the system. Examples include (i) articulating that improving employee motivation and counselling are supervisors' functions (Shafie 1996); (ii) emphasising developmental feedback (Kim 2014); and (iii) using rewards based on both individual performance and the team's performance to encourage honest feedback from team members (Jiang 2010).

\subsubsection{Performance-based reward}

Performance-based reward refers to rewarding individuals according to their performance and contribution (Gavish, Gerdes et al. 2000; Armstrong 2002). Connecting performance to rewards such as pay, promotion and recognition is regarded an integral part of PM because employees generally feel more motivated when their performance is rewarded (Lawler and McDermott 2003; Chiang and Birtch 2010; Azzone and Palermo 2011).

Reward-for-performance is supported by a number of theories, such as expectancy, equity and reinforcement. These theories focus respectively on the desirability and achievability of rewards, comparisons with others to estimate the fairness of effort/reward ratios, and the contingency between achievement and reward (Skinner 1953; Adams and Rosenbaum 1962; Vroom 1964). In a study conducted in 55 Fortune 500 companies, Lawler (2003) found that reward-for-performance does not decrease the effectiveness of employee $\mathrm{PM}$, but makes it more effective as a tool to get employees and organisations committed to appraising and rewarding performance well. This is congruent with Van Herpen, Van Praag et al.'s (2005) findings that reported that performance-based rewards (compensation and promotion) relate significantly to employee motivation and job satisfaction. In the public service, the introduction of pay-for-performance might improve employee motivation and 
enhance the efficiency of the public sector (Burgess and Ratto 2003; Lavy 2007; Stazyk 2009).

In contrast, the lack of such a connection makes individuals less committed and interested in the PM system (Dzimbiri 2008). A study on employee PM in the developing context concluded that "until a system for rewarding better performers is put in place, the PMS (performance management system) will not work" (Ohemeng 2009 p123).

To date, two-thirds of OECD members and many countries in the Asia Pacific have introduced or been in the process of adopting contingent pay systems (Shafie 1996; Perry, Engbers et al. 2009; Kong, Kim et al. 2013). However, sceptics argue that inherent characteristics of the public sector such as conflicting goals, conflict with public service motivation, the need for transparency in pay, inadequate budget and union opposition make pay-for-performance goals very difficult to achieve (Verbeeten 2008; Perry, Engbers et al. 2009; Weibel, Rost et al. 2009; Taylor and Beh 2013). In fact, pay for performance has a very patchy record in governmental organisations (Plimmer, Bryson et al. 2017) as well as private organisations. Arguably, the introduction of pay-for-performance is likely to cause unwanted consequences such as constraining creativity, focussing on short-term goals, increasing political motives, undermining teamwork morale, weakening intrinsic motivation and distracting people from the meaningfulness of the job (Pfeffer 1998; Weibel, Rost et al. 2009; McShane and Travaglione 2010).

Adoption of pay-for-performance is even more challenging in the public sector in developing countries because capacity problems and the linkage of appraisal results with pay may not "fit" culturally with group norms of harmony and collectivism (Chiang and Birtch 2010; Kong, Kim et al. 2013). Instead of relying on performance, rewards are often based on seniority, political loyalty and personal (patronage) relationships (Tong, Straussman et al. 1999; Siddiquee 2003; Randma-Liiv 2005; Seniwoliba 2014; Harbi, Thursfield et al. 2017). Consequently, many pay-for-performance schemes in the public sector have not created expected outcomes.

However, this failure may be attributed to implementation issues rather than the failure of theory (Randma-Liiv 2005). Empirical research, such as that done by McShane and Travaglione (2010) concurs that "employees with better performance should be rewarded more than those with poor performance...top-performing companies are more likely to have performance-based rewards" (p.212). In a study in Portugal, Cunha, Vieira et al. (2018) 
argue that performance-based reward is key to engaging employees in the PM system as well as creating alignment between individual goals and the organisation's goals. In a survey with US city managers, assistant city managers and department heads, Stazyk (2009) finds that employees are more satisfied when performance-related pay is present. In another survey with UK National Health Service managers, Dowling and Richardson (1997) find positive effect of performance-related pay on the motivation and effort of managers, albeit at a modest level.

Additionally, empirical research in developing countries suggests that reward systems are more likely to be effective in improving employee performance and customer satisfaction under the following conditions:

1) Rewards are based on both individual performance and the team's performance (Koonmee 2009).

2) Rewards are large enough to attract interest from employees (Kong, Kim et al. 2013).

3) Pay is the combination of base salary and performance pay (Shafie 1996; Kong, Kim et al. 2013).

4) There is a combination of both financial rewards and non-financial rewards such as recognition, training opportunities, and career development opportunity to meet diverse needs and reduce the cost burden (Deci and Ryan 2000; Kong, Kim et al. 2013).

\subsubsection{Dealing with poor performers}

Good PM systems ensure that not only are good performers rewarded but also that poor performers' problems are properly resolved (Tong, Straussman et al. 1999; Luecke and Hall 2006; Dzimbiri 2008). Poor performers generally refer to employees who fail to fulfil the job at an acceptable level in terms of quality, quantity and timeliness US.OPM (2002), as cited in (Parham 2003). Poor performers make lacklustre decisions, implement programmes poorly, and set bad examples to colleagues. Good performers feel resentful when poor performers "get the same rewards and keep their jobs without making proportionate contributions" (Leavitt and Johnson 1998 p76). Furthermore, poor performers tend to be gossip mongers, complainers and mean-spirited jokesters who undermine the trust and solidity of the members and then disrupt the workplace (Luecke and Hall 2006). Failure 
to address repeated poor performers might lead to unproductive behaviours and demotivate good performers.

In contrast, dealing with poor performance through measures such as feedback, formal warnings, provision of opportunities to improve, criticism, transfers and even termination is likely to limit the above negative consequences (O'Reilly III and Weitz 1980; Stoeberi and Schniederjans 1981; Schwartz 1995; Luecke and Hall 2006). Research indicates that if properly applied, this practice might increase commitment and performance by changing unwanted behaviours, improving attendance, developing performance culture, removing obstacles to achievement, removing a source of inequity, and then reinforcing public service values (O'Reilly III and Weitz 1980; Bridges 1985; Leavitt and Johnson 1998; Lawler 2003). In a study on the employee PM system in the developing context, Ohemeng (2009) concludes that "a good incentive and sanction system is imperative for effective PM" (p.123).

Undeniably, all civil service systems have poor performers regardless of good HR practices in place (Schniederjans and Stoeberl 1986; Tong, Straussman et al. 1999; Colli 2013). However, this problem is often more serious and prevalent in developing countries because of obsolete personnel practices and historical and cultural factors (Tong, Straussman et al. 1999; Jacobs 2004; Poon, Hung et al. 2009). Specifically, as well as rigid employee protections, the pervasiveness of personal relationships, nepotism, collectivism, and corruption makes dealing with poor performers in the public sector in developing countries difficult (Cooke 2003; Randma-Liiv 2005; Poon, Hung et al. 2009; Ohemeng, Zakari et al. 2015). Additionally, traditional values and welfare systems where one is supposed to take care of family members, particularly elderly parents impedes the application of hard measures on poor performers (Ohemeng 2009).

However, given increasing pressure on the public service to improve efficiency and transparency, and the austerity crisis, the last two decades have witnessed some changes in the ways poor performers are handled in developing countries. For example, in China, central ministries are responsible for providing retraining programmes for poor performing employees to help them improve to keep working in the public sector (Cooke 2003). However, the likelihood of improvement is limited if the employee does not have either the desire or ability to improve (Blacklock 2002). Consequently, a number of former socialist countries have begun reforming the lifelong employment regimes and have allowed public 
organisations more flexibility in dismissing poor employees (Tong, Straussman et al. 1999; Cooke 2003; Meyer-Sahling 2012).

Although such change is an important start in dealing with poor performance, the result is mixed due to the risk of grievance (or even litigation), favouritism, and politicisation. The problems are even more difficult when the appraisal systems do not function properly (Meyer-Sahling 2012). Therefore, another advisable measure used in this research is to transfer poorly performing employees to other positions that better match their competence before proceeding to termination procedures if low performance continues. Some studies have pointed out that transfer is the most frequently used measure by managers when addressing poor performing employees (Stoeberi and Schniederjans 1981; Ehrgott 1993). This not only mitigates litigation-related tension, but also consolidates work morale by signalling that the organisation will no longer tolerate poor performers (Osborne and Plastrik 1997; Tong, Straussman et al. 1999).

\subsubsection{Employee participation}

Employee participation in the employee PM process refers to participation between employees and their supervisors in (i) goal setting; (ii) the development of performance standards; (iii) appraisal interview and (iv) discussion of the rating result (Roberts 2003). Employee participation is an essential component of employee PM because it increases employees' cognitive ability by encouraging open communication, expressing new ideas, and sharing common purposes (Fletcher and Williams 1996; Roberts and Reed 1996). These all intensify mutual respect and trust (Rodgers and Hunter 1991; Shafie 1996; Nyhan 2000). Participation can arguably mitigate against many dysfunctional aspects of traditional appraisal (Roberts 2003). For example, open discussions with supervisors clarify goals and standards of performance while increasing the constructiveness of feedback and the accuracy of performance ratings. Employee participation enables other employee PM practices (e.g. goal-based appraisals and performance-based rewards) to be more effective because it helps clarify goals and performance standards, aligns contributions, increases constructive feedback, and improves the accuracy of performance ratings (Roberts 2003).

Arguably, employees are more motivated and satisfied when they have been encouraged to discuss their performance as well as how rewards will be distributed. This is because employees feel they have more control of the process and they gain respect because their opinions have been taken into account (Posthuma and Campion 2008). Encouraging 
employee participation in goal setting and feedback signifies that the supervisor appreciates the employee's contribution and commits to help him/her obtain desirable goals. As suggested by social exchange theory, employees will reciprocate by attempting to contribute to the organisation (Parzefall and Salin 2010). Additionally, employee participation fosters employees' sense of trust, ownership, self-efficacy, satisfaction, and responsibility, thereby reinforcing acceptance and encouraging initiatives, cooperation and commitment in fulfilling organisational goals (Nyhan 2000; Roberts 2003; Kleingeld, Van Tuijl et al. 2004).

Despite the strong case in western countries for participation in decision-making, this practice is often impeded in developing countries (Shafie 1996; Milikić, Janićijević et al. 2012). For many of these countries, the process of centralised decision-making and the culture of high power distance strongly orients managers towards superiors and formal rules rather than using inputs from subordinates (Hofstede 2001). Besides, cultural patterns of obedience, fatalism and paternalism make employees passive and reluctant to take initiatives and responsibility (Aycan, Kanungo et al. 2000; Munene, Schwartz et al. 2000; Zientara and Kuczyński 2009). In such contexts, employee participation might not arise easily unless there are interventions e.g. training, communication or specific regulations. For example, in the innovation process of the employee PM practice, the governments of Malaysia and Korea emphasised the requirement to get employees involved in the PM process such as goal setting, mid-year reviews and annual appraisals (Shafie 1996; Kong, Kim et al. 2013). They also provided training courses to help managers understand their roles in the implementation of employee PM aimed at stimulating democratic working environments and employee participation. Meanwhile, in Vietnam, in the innovation process of employee PM, some organisations encourage employees to participate in developing performance standards and ways to distribute extra income to members of the team. Table 3-1 summarises the above discussion (with references).

Table 3-1: Employee PM practices and issues in developing countries

\begin{tabular}{cll}
\hline $\begin{array}{c}\text { Employee PM } \\
\text { practices }\end{array}$ & \multicolumn{1}{c}{ Key issues/impediments } & References \\
\hline & $\begin{array}{l}\text { Weak accountability, ambiguous missions } \\
\text { and frequently changing priorities }\end{array}$ & $\begin{array}{l}\text { Randma-Liiv (2005) and Liu and Dong } \\
(2012)\end{array}$ \\
$\begin{array}{l}\text { Goal-based appraisal } \\
\text { (aligning individual } \\
\text { contribution with } \\
\text { organisational goals) }\end{array}$ & $\begin{array}{l}\text { Political influence, and current regulations } \\
\text { that emphasise abstract appraisal criteria }\end{array}$ & $\begin{array}{l}\text { Liu and Dong (2012), and Koubek and } \\
\text { such as political ideology, loyalty, }\end{array}$ \\
\hline
\end{tabular}




\begin{tabular}{|c|c|c|}
\hline & $\begin{array}{l}\text { Egalitarianism, collectivism and process- } \\
\text { oriented cultures, fatalism and high } \\
\text { uncertainty avoidance }\end{array}$ & $\begin{array}{l}\text { Poon, Hung et al. (2009), Stanton and Pham } \\
\text { (2014), Koubek and Brewster (1995), } \\
\text { Mendonca and Kanungo (1996), and } \\
\text { Ohemeng (2009) }\end{array}$ \\
\hline & Interpersonal relationships and nepotism & $\begin{array}{l}\text { (Koubek and Brewster 1995; Liu and Dong } \\
\text { 2012) and Tong, Straussman et al. (1999) }\end{array}$ \\
\hline & $\begin{array}{l}\text { Lack of ability to set objective goals, } \\
\text { performance standards and job descriptions }\end{array}$ & $\begin{array}{l}\text { De Waal (2007), Liu and Dong (2012), } \\
\text { Randma-Liiv (2005) and Koubek and } \\
\text { Brewster (1995) }\end{array}$ \\
\hline \multirow{5}{*}{$\begin{array}{l}\text { Feedback } \\
\text { (motivating, directing } \\
\text { and instructing } \\
\text { employees) }\end{array}$} & $\begin{array}{l}\text { Conflict with current personnel policies e.g. } \\
\text { democratic appraisal from subordinates for } \\
\text { re-promotion of supervisors }\end{array}$ & $\begin{array}{l}\text { Liu and Dong (2012), and Burns and Xiaoqi } \\
\text { (2010) }\end{array}$ \\
\hline & Job-unrelated appraisal criteria & $\begin{array}{l}\text { Vallance (1999) and Koubek and Brewster } \\
\text { (1995) }\end{array}$ \\
\hline & Confucianism and face-saving culture & $\begin{array}{l}\text { Hofstede (1998) and Chiang and Birtch } \\
\text { (2010) }\end{array}$ \\
\hline & Lack of employee input and participation & Chiang and Birtch (2010) \\
\hline & Untrained supervisors & Randma-Liiv (2005) \\
\hline \multirow{6}{*}{$\begin{array}{c}\text { Reward for } \\
\text { performance } \\
\text { (directing and } \\
\text { motivating employees } \\
\text { to achieve individual } \\
\text { and organisational } \\
\text { goals) }\end{array}$} & $\begin{array}{l}\text { Financial constraints and overstaffing in the } \\
\text { public sector }\end{array}$ & $\begin{array}{l}\text { Ohemeng (2009), Randma-Liiv (2005), } \\
\text { Meyer-Sahling (2012) and Siddiquee } \\
(2003)\end{array}$ \\
\hline & Secretive promotion processes & Tong, Straussman et al. (1999) \\
\hline & $\begin{array}{l}\text { Buying and selling government posts and } \\
\text { patronage }\end{array}$ & $\begin{array}{l}\text { Burns and Xiaoqi (2010), Burns and Zhiren } \\
(2010) \text { and Teclemichael Tessema and } \\
\text { Soeters (2006). }\end{array}$ \\
\hline & Seniority-based pay & $\begin{array}{l}\text { Burns and Zhiren (2010), Meyer-Sahling } \\
\text { (2012) and Zientara and Kuczyński (2009) }\end{array}$ \\
\hline & Interpersonal relationships & $\begin{array}{l}\text { Law, Wong et al. (2000) and Tong, } \\
\text { Straussman et al. (1999) }\end{array}$ \\
\hline & Egalitarianism & $\begin{array}{l}\text { Stanton and Pham (2014), Koubek and } \\
\text { Brewster (1995) and Burns and Zhiren } \\
(2010)\end{array}$ \\
\hline \multirow{5}{*}{$\begin{array}{l}\text { Dealing with poor } \\
\text { performers } \\
\text { (removing sources of } \\
\text { inequity, changing } \\
\text { unwanted behaviours, } \\
\text { improving attendance, } \\
\text { and removing obstacles } \\
\text { to achievement) }\end{array}$} & $\begin{array}{l}\text { Unreliable appraisal results due to the lack } \\
\text { of objective and job-related criteria }\end{array}$ & Siddiquee (2003) and Liu and Dong (2012) \\
\hline & Rigid employee protection & $\begin{array}{l}\text { Cooke }(2003) \text { and Teclemichael Tessema } \\
\text { and Soeters }(2006)\end{array}$ \\
\hline & $\begin{array}{l}\text { Corruption in recruitment and colluding } \\
\text { with subordinates to carry out corrupt } \\
\text { activities }\end{array}$ & Poon, Hung et al. (2009) \\
\hline & Nepotism and personal relationships & $\begin{array}{l}\text { McCourt and Ramgutty-Wong (2003) and } \\
\text { Bozionelos and Wang (2007) }\end{array}$ \\
\hline & $\begin{array}{l}\text { Traditional culture where one is supposed } \\
\text { to take care of elderly parents }\end{array}$ & Ohemeng (2009) \\
\hline \multirow{3}{*}{$\begin{array}{c}\text { Employee } \\
\text { participation } \\
\text { (clarifying goals, } \\
\text { aligning contributions, } \\
\text { increasing constructive } \\
\text { feedback and trust) }\end{array}$} & Secret evaluation & $\begin{array}{l}\text { Siddiquee (2003) and McCourt and } \\
\text { Ramgutty-Wong (2003) }\end{array}$ \\
\hline & $\begin{array}{l}\text { High power distance, } \\
\text { management style }\end{array}$ & $\begin{array}{l}\text { Hofstede (2001), Stanton and Pham (2014) } \\
\text { and Zientara and Kuczyński (2009) }\end{array}$ \\
\hline & Fatalism & Mendonca and Kanungo (1996) \\
\hline
\end{tabular}

\subsection{Outcomes of employee performance management and underpinning theories}

Murphy and Cleveland (1995) suggest that a good PM system is one that enables the stakeholders to achieve their objectives. Normally, the effectiveness of a performance appraisal system largely relies on how well it satisfies goals such as providing useful and 
accurate information for administrative decisions about promotion, employee development, transfers, discipline, and layoffs (Jacobs, Kafry et al. 1980; Evans and McShane 1988). Meanwhile, the effectiveness of an employee PM system is evaluated by its ability to improve individual performance and subsequently organisational performance (Haines III and St-Onge 2012; Abbas 2014).

Boselie, Dietz et al. (2005) argue that employee PM as part of HRM systems is designed to achieve both HRM outcomes (e.g. employee satisfaction and motivation, organisational commitment) and organisational-level outcomes (e.g. productivity, service quality and customer satisfaction). The literature suggests that its objective is not only evaluated by how well it helps managers understand employees' strengths and weaknesses but also by its capacity to improve work morale, recent efforts on the job, motivation, job satisfaction, organisational commitment and productivity (Murphy and Cleveland 1995; Fletcher and Williams 1996; Taylor and Pierce 1999; Mani 2002). One study in the public sector in England reported that the main objectives of implementing employee PM in public organisations include: improving employee proficiency; raising levels of employee motivation and accountability; improving employee efficiency and performance, and increasing organisational efficiency and customer service (Brown 2005). Based on these suggestions, the following section presents expected outcomes of employee PM, and the theories that explain how its introduction can produce those outcomes.

\subsubsection{Employee motivation}

As discussed above, one of the most desired objectives of employee PM is to increase employee motivation which is defined as "a set of energetic forces that originates both within as well as beyond an individual's being to initiate work-related behaviours, and to determine its form, direction, intensity and duration" (Pinder 2014, p.11). There is an argument that while intelligence and competence can affect performance, employee motivation is mainly responsible for differentiating superior from ordinary performers (McClelland 1973; Dubois and Rothwell 2004; Shields 2007). The relationship between employee PM and employee motivation can be explained by goal setting theory and expectancy theory.

According to goal-setting theory, employee motivation will be strengthened if the goals are specific and challenging but feasible (Locke 1968). Specifically, the more challenging and specific the goals are, the more effort employees put into their jobs. As a 
key component of employee PM, setting measurable goals at the beginning of the performance period enables employees to concentrate on the achievement of critical objectives, increase effort and overcome the challenges (O'Boyle and Cummins 2013). In this vein, Walker, Damanpour et al. (2010) argue that performance management is an effective mechanism to tackle the problem of ambiguous and conflicting goals in the public sector. Additionally, goal setting with employee participation enables employees to have more voice and autonomy in their performance, thereby increasing motivation.

From another perspective, expectation theory suggests that a person's motivation depends on (i) the probability of accomplishing the goals; (ii) the probability of receiving rewards once the goals are achieved; and (iii) the value of rewards (Vroom 1964). Therefore, the introduction of employee PM is aligned with this theory. For example, goal setting with information input from employees ensures that the goals are feasible and achievable while the practice of reward-for-performance enables employees to see the link between their efforts and rewards, which in turn increases work motivation. (Roberts and Reed 1996; Taylor and Pierce 1999).

\subsubsection{Organisational commitment}

Increasing organisational commitment should be viewed as a desirable outcome of an employee PM system because it is supposed to lead to higher motivation and individual performance (Fletcher and Williams 1996). Organisational commitment may be understood as an employee's belief and trust in organisational values and objectives, and affection towards the organisation (Mowday, Porter et al. 1982). Porter, Steers et al. (1974) contend that organisational commitment has at least three elements, including "(i) a strong belief in, and acceptance of the organisation's goals and values; (ii) a willingness to exert considerable effort on behalf of the organisation; and (iii) a definite desire to maintain organisational membership" (Porter, Steers et al. 1974, p.3). As such, an employee with high organisational commitment will show active and voluntary participation in order to fulfil the organisation's goals (Mowday, Steers et al. 1979). Indeed, in a report on employee engagement, MacLeod and Clarke (2011) contend: "Business and organisations function best when they make their employees' commitment, potential, creativity and capability central to their operation. Clearly, having enough cash, and a sensible strategy, are vital. But how people behave at work can make the crucial difference between business and operational success or failure." (p.7). 
Research indicates that the components of employee PM can increase organisational commitment in a number of ways (Fletcher and Williams 1996; Meyer, Allen et al. 1997). For example, reward-for-performance and the provision of constructive feedback will develop organisational commitment as a form of reciprocity towards the organisation (Steers 1977; Kim, Price et al. 1996; Meyer, Allen et al. 1997). This relationship is explained by social exchange theory that suggests once employees are exposed to a favourable work environment and favourable benefits, they will feel obligated to reciprocate by adopting a more positive attitude (commitment) toward the organisation (Steers 1977; Meyer, Allen et al. 1997; Ko and Hur 2014).

In this regard, Parzefall and Salin (2010) contend that employees who perceive that the organisation has a true commitment to helping them obtain their socio-emotional and tangible desires will reciprocate by helping the organisation to obtain its objectives. In addition to reward and constructive feedback, encouraging employee participation in the PM process such as goal setting and feedback signals that the organisation values and is committed to its employees. This, in turn, increases the employee's organisational commitment in exchange for the organisation's support (Eisenberg, Fasolo et al. 1990; Rhoades, Eisenberger et al. 2001).

\subsubsection{Job satisfaction}

People who are more satisfied with their jobs perform better and exhibit more organisational citizenship behaviours (Schmit and Allscheid 1995; Whitman, Van Rooy et al. 2010). Improving job satisfaction should be one of the most desired outcomes when designing an employee PM system (Fletcher and Williams 1996; Fletcher 2001; Boselie, Dietz et al. 2005). Job satisfaction is understood to be the "pleasurable or positive emotional state resulting from the appraisal of one's job or job experience" (Locke 1976, p.1300). Spector (1994) defines job satisfaction as "the extent to which people like (satisfaction) or dislike (dissatisfaction) their jobs" (p.2). In theory, the introduction of employee PM can help the organisation heighten employees' job satisfaction in a number of ways.

First, employee PM tends to apply merit principles that reward good performers based on their contribution. This supposedly increases the equity in the organisation, which in turn heightens job satisfaction and employee motivation (Risher 2002). The equity theory suggests that employees tend to compare the ratio between their inputs (effort or contribution) and outputs (rewards) with that of their colleagues and use that as the basis for 
evaluating equality (Adams and Rosenbaum 1962; Adams 1963). Once employees perceive that their reward is equal to other employees' for similar contributions, they will believe they are being fairly and equitably treated which in turn leads to job satisfaction (Dawis 2004; Spector 2008). Additionally, addressing poor performers properly can also increase job satisfaction because it helps to remove a source of inequity (O'Reilly III and Weitz 1980).

Second, through goal setting and employee participation, employee PM gives employees more autonomy, responsibility and independence in performing their tasks. In a meta-analysis, Spector (1986) finds that autonomy significantly relates not only to job satisfaction but also to organisational commitment, motivation and performance. Besides, goal-based appraisal increases job satisfaction by clarifying the organisation's expectations and perception of their contributions (Locke 1976; Ting 1997; Pettijohn, Pettijohn et al. 2001; Kalgin, Podolskiy et al. 2018).

Third, unlike traditional appraisals, employee PM enables managers to implement fair and objective ratings as well as providing useful feedback and coaching to improve employee performance. This, in turn, increases employees' trust in, and satisfaction with the managers (Mayer and Davis 1999).

Finally, by creating the opportunity for open communication and participation in decision making, employee PM helps employees feel fulfilled (i.e. social belonging and esteem), which in turn intensifies job satisfaction (Wheeless, Wheeless et al. 1984).

\subsubsection{Organisational performance}

Aguinis (2009) states that "an important objective of any performance management system is to enhance each employee's contribution to the goals of the organisation" (p.38). Arguably, the implementation of employee PM can increase individual performance, which in turn increases organisational performance through different channels. First, Taylor and Pierce (1999) contend that goal setting helps employees to clarify their role and move towards the organisation's goals. In an employee PM system, employees are managed based on the principle of agreement rather than command. This allows employees to have more flexibility to do their best work to obtain the desirable results (Dzimbiri 2008).

Second, feedback that is timely, specific and based on credible sources helps poor performers to correct their behaviours promptly whilst helping good performers to continue to excel by providing positive reinforcement (Somerick 1993). Supporting this argument, 
organisational learning theory proposes that learning organisations will encourage open and honest conversations between organisational members. This, in turn, enables employees to receive useful and constructive feedback about how to improve their performance as well as encouragement to continue with positive behaviours (Walsh and Fisher 2005).

Third, backed up by reinforcement theory (Skinner 1953), reward-for-performance is most likely to enhance employee performance because it enables the modification of employee behaviours according to the organisation's desire. Specifically, employees tend to repeat behaviours that lead to pleasant consequences, while removing behaviours that lead to unpleasant consequences.

Fourth, dealing with poor performance appropriately is likely to increase commitment and performance by removing a source of inequity, changing unwanted behaviours, improving attendance, removing obstacles to achievement and then reinforcing organisational performance (O'Reilly III and Weitz 1980; Bridges 1985; Leavitt and Johnson 1998).

Finally, via the intermediate role of employee motivation, organisational commitment and job satisfaction, employee PM can affect organisational performance. Meta-analyses conclude that employee motivation (Cerasoli, Nicklin et al. 2014), organisational commitment (Randall 1990) and job satisfaction (Judge, Thoresen et al. 2001) positively correlate with employee performance and subsequently organisational performance. Similarly, a body of research asserts that employees with high motivation, organisational commitment and job satisfaction show active and voluntary participation in order to fulfil the organisation's goals (Mowday, Steers et al. 1979; Fletcher 2001; Whitman, Van Rooy et al. 2010).

This research uses Vietnam as a transitional economy to examine whether employee PM can be successfully introduced in the developing context. More specifically, it examines whether the introduction of advanced employee PM practices can be conducive to increased work motivation, organisational commitment, job satisfaction and subsequently organisational performance. In addition, it explores which specific PM practices and implementation strategies, if any, can be used in a developing context to obtain these outcomes. These outcomes are critical for developing countries where many ambitious reform programmes have been disrupted by unmotivated, incompetent and unethical officials (Tong, Straussman et al. 1999; Poon, Hung et al. 2009). 
Based on the above discussion, the research formulates that:

Hypothesis 1: Employee PM is associated with increased perceptions of employee motivation (Hla), organisational commitment (H1b), job satisfaction (H1c) and organisational performance (HId).

\subsection{The effect of contextual factors on employee performance management}

Although there has been a large amount of research into the techniques and processes of employee PM, little has been translated into effective PM systems (Kozlowski, Chao et al. 1998). Murphy and Cleveland (1995) called for researchers to pay more attention to contextual factors since the outcomes are most likely to be shaped by the context in which employee PM takes place. However, there is still limited knowledge about what factors foster or hinder the introduction, institutionalization and success of employee PM, particularly in the public sector (Carassus, Favoreu et al. 2014). Nor do we know much about what determines the effectiveness of an employee PM system (Decramer, Smolders et al. 2012). Many well-invested PM schemes fail because the context was not sufficiently taken into account (Marshall and Wood 2000; Haines III and St-Onge 2012), and many scholars argue that the effectiveness of HRM practices must be congruent with the social-cultural context in which they are conducted (Mendonca and Kanungo 1996; Aycan 2005; Ohemeng 2009).

Irrespective of calls to pay attention to contextual factors, the literature still lacks a clear and cohesive understanding of the degree, pattern, and circumstances under which contextual variables affect employee PM (Murphy and Cleveland 1995; Decramer, Smolders et al. 2012; Pichler 2012). The need for research into contextual factors has become even more necessary in developing countries when practitioners unexpectedly discover that many programmes that have been successful in industrialised countries are failing in their countries, which have different socio-cultural and institutional environments (Mendonca and Kanungo 1996; Aycan, Kanungo et al. 2000). In order to narrow the gap in the literature, the present research reviewed contextual factors driving the introduction and implementation of employee PM in developing countries. Then the most influential factors were selected for detailed investigation.

This study extends our knowledge of context by examining four factors that, the author believes, may strongly affect the implementation of employee PM in developing countries. These factors are also subject to intervention (i.e. change). The first three factors concern 
institutional issues to break away from traditional appraisal processes and adopt employee PM, namely agency accountability, HR autonomy and entrepreneurial leadership. The fourth factor concerns the capacity of the organisation to muster the necessary HR expertise to transform existing processes into employee PM-based processes. Additionally, this research examines the role of two cultural variables of nepotism and interpersonal relationships in the development of employee PM. The following section will discuss the role of these factors in turn.

\subsubsection{Institution and capacity factors}

\subsubsection{Agency accountability}

Agency accountability refers to the specific expectations and mechanisms by which public managers are called to account for their organisations' performance (Marshall and Wood 2000). Normally, such accountability is provided by senior leaders e.g. ministers, as well as higher organisations, e.g. oversight agencies and legislatures. Agency accountability is often held through establishing performance goals, measuring performance with measurable criteria, and connecting performance with bonuses or sanctions. Increased accountability not only makes the executives' responsibility for the organisation's performance more explicit but also ties their fortune (including career advancement) with the organisation's performance.

Although the relationship between accountability and employee PM is underresearched (Agyemang and Ryan 2013), this research assumes that increased accountability will promote the development of employee PM. This is because accountability demands usually aim to promote organisational changes for performance improvement (Moynihan and Pandey 2004) which in turn forces or motivates chief executives to devise standards, tools and procedures that can be instrumental in obtaining desired outcomes (Heinrich and Marschke 2010).

Among these likely tools, the employee PM system can be used to attain the organisation's goals through improving individual performance and has been advocated by researchers and practitioners (Longenecker and Goff 1992; Shafie 1996; Coens and Jenkins 2002; Agyemang and Ryan 2013). In this vein, Haines III and St-Onge (2012) argue that "When human capital is value, the performance management system may deliver its full potential" (p.1170). It has been said that accountable executives are more likely to engage 
in setting clear objectives, monitoring performance, providing constructive feedback and using rewards and sanctions for their subordinates (O'Reilly III and Weitz 1980; RandmaLiiv 2005; Ho and Im 2015).

Regrettably, given weak institutions and the limited capacity of upper-level authorities, the accountability of chief executives in the developing world is regarded as weak and deficient (Lerner and Tetlock 1999). This problem also derives from the collective leadership mechanism as a feature of former communist nations. Arguably, though the collective leadership mechanism can help maintain stability, it constrains radical reforms. On this issue, Ketels, Nguyen et al. (2010) and Liu and Dong (2012) argue that weak accountability and the collective leadership mechanism together slow down necessary reforms and employee PM innovation is no exception.

While the problems are serious and often noted (Burns and Zhiren 2010; Liu and Dong 2012; Ho and Im 2015), reform trends are towards greater transparency, better scrutiny, more effectiveness and improvement in performance. For example, in an effort to reform the public service, the governments of Korea and Indonesia, and some provinces in China, have attempted to establish performance measurement systems at the organisational level to enhance accountability and organisational performance (Burns and Zhiren 2010; Akbar 2011; Kong, Kim et al. 2013). Similarly, some provinces in Vietnam have begun evaluating chief executives through the organisation's performance (BacGiang 2013; Danang 2014). Although the overarching effect of such reforms is still unclear, they do promote the introduction of a wide range of new PM practices such as cascading the organisation's goals to individual goals, goal-based appraisal, providing feedback and linking performance with rewards and consequences (Burns and Zhiren 2010; Liu and Dong 2012; BacGiang 2013; Ho and $\operatorname{Im} 2015)$.

\subsubsection{Agency autonomy in HRM practice}

Public organisations in transitional economies often have less administrative autonomy than their counterparts in developed countries, particularly when central planning is embedded (Zientara and Kuczyński 2009). In the Soviet-style management model, central authorities decided the detailed plans of subordinate organisations. It was necessary and compulsory to get approval from upper-level authorities for any change (Koubek and Brewster 1995). For such bureaucratic and hierarchical administrations, innovations without higher agencies' permission can cause risks for the initiators (Linz and Stepan 1996). Unlike 
private sector enterprises that can do whatever laws do not prohibit, public sector organisations are only allowed to do what laws and higher agencies permit. An initiative exceeding the granted authority can be seen as a violation. Arguably, an institutional and legal framework determines the extent to which a public organisation can pursue management innovation (Birkinshaw, Hamel et al. 2008).

Like any other innovation initiative in the public sector, employee PM innovation will be promoted if it is supported and given more autonomy to do so. Agency autonomy allows organisations to have more "space" and confidence to introduce new policies aimed at replacing obsolete and incompatible ones. For example, in the reform process in China, while central agencies still continue to adhere to traditional personnel management practices which often rely on seniority and egalitarianism (Liu and Dong 2012), local governments, with more autonomy, have devised several initiatives aimed at reforming employee PM. These initiatives often entail formulating output-based appraisal criteria, linking performance with financial rewards, career advancement and dismissing underperforming employees (Burns and Zhiren 2010). Similarly, research in the fields of education and health points out that the institutions granted more autonomy tend to take into account their particular conditions and adopt HR policies that are more compatible with the needs of the organisation, thereby increasing staff commitment and better meeting customer needs (Redman, Snape et al. 2000; Sanwong 2008; Dou, Devos et al. 2016). As such, autonomy, particularly HR autonomy is seen as an antecedent for the radical innovation of employee PM.

\subsubsection{Entrepreneurial leadership}

The role of leadership in implementing employee PM has been researched, usually in terms of the role of leaders to communicate goals, provide resources, express commitment and role model during implementation (Marshall and Wood 2000; Tung, Baird et al. 2011; Tuytens and Devos 2012). Very little research has investigated the leadership quality which makes a senior public manager proactively trigger innovation initiatives (Carassus, Favoreu et al. 2014). With the aim to narrow this gap, this study investigates the relationship between entrepreneurial leadership and the advancement of employee PM.

Compared to ordinary leaders, entrepreneurial leaders possess characteristics such as vision, a sense of urgency, innovation, risk-taking, resilience, provision of motivation and dedication to superior performance (Zahra and Covin 1995; Thornberry 2006). In the public 
sector, entrepreneurial leaders take initiative, pursue innovations, tackle enduring problems and achieve improved public sector outcomes (Kearney, Hisrich et al. 2009; Berman, Chen et al. 2017). Although the relationship between entrepreneurial leadership and employee PM is under-researched to date, we can predict a close-knit relationship between them, particularly in the developing context. The reason is that public managers in these countries usually operate under the setting of obsolete personnel management mandates, bureaucratic culture, and limited managerial autonomy as mentioned above. Consequently, the innovation of employee PM in the transition period is likely to face challenges and risks (De Waal 2007; Kong, Kim et al. 2013). In such circumstances, ordinary managers tend to avoid taking initiatives so as not to become "scapegoats" for the ineffectiveness of the system (Suutari and Riusala 2001).

However, for executives with entrepreneurial leadership, the shortcomings of the current PM systems (traditional appraisal systems) are viewed as obstacles or urgent issues that need to be solved to create a more effective operating state. Dissatisfaction with the current state of affairs makes them willing to go beyond the current regulations or boundaries to reform obsolete appraisal systems by innovating abstract appraisal criteria, encouraging employee participation and seeking ways to link performance with consequences. A number of studies have concurred that the authentic determination and persistence of top managers play a substantial role in the development of PM systems (Boice and Kleiner 1997; Holloway, de Waal et al. 2009; Tung, Baird et al. 2011). They support innovation by persuading stakeholders to support innovation initiatives, allocating resources, solving legal issues, rewarding those who help make the system successful and dealing with those who are uncooperative and destructive (Allan 1994; Boice and Kleiner 1997; Marshall and Wood 2000; Lawler, Benson et al. 2012).

\subsubsection{HR competence}

The majority of employee PM systems are designed and implemented by internal HR professionals (Gorman, Meriac et al. 2017). The extent to which HR professionals master HRM competencies will affect the development of the employee PM system (Roberts and Pavlak 1996; Terpstra, Mohamed et al. 1996; Galang 1999). HR competence concerns effective HR practices that help the organisation achieve desired goals (Selmer and Chiu 2004). A frequently cited set of competencies is that proposed by Ulrich, Brockbank et al. (1995). Accordingly, to perform HR functions effectively, HR professionals need to have 
(i) insight into the organisation's operations (knowledge of business); (ii) expertise (knowledge and skills) in the HRM area; and (iii) competency to manage change processes.

The following reasons may explain the effect of HR competence on the advancement of employee PM. First, HR professionals' insights into the organisation's current situation and strategic goals will help the PM system to better connect to strategic goals, thereby obtaining senior managers' support (Legge 1978). Second, as one of the major actors in designing and implementing PM systems, HR professionals must have expertise and proficiency in the HRM area e.g. knowledge of motivation theories and skills about job analysis, appraisal, development, rewarding and handling difficult employees. This expertise is essential to ensure the effective operation of an employee PM system (Terpstra, Mohamed et al. 1996). Third, innovating employee PM is viewed as a change management process. Possessing change management competency can minimise resistance, increase employees' acceptance and reinforce desired behaviours. Based on these rationales, Rao (2004) states that "The successful implementation of new appraisal systems also depends to a great degree on the personnel and HRD department. The responsibility for initiating and monitoring the implementation of a new appraisal system is usually given to these departments" (p.181). Accordingly, an organisation tends to introduce more effective and workable HRM practices when HR professionals are competent and understand effective HRM system characteristics (Roberts and Pavlak 1996; Terpstra, Mohamed et al. 1996; Murphy and Southey 2003; Ngo, Jiang et al. 2014).

Nevertheless, personnel units in transitional countries usually lack HRM knowledge and relevant experience to do this strategic work and are instead reactive administrators (Taylor 1992; Tong, Straussman et al. 1999; Poon, Hung et al. 2009). Personnel units are virtually administrative units with little decision-making power (Koubek and Brewster 1995). For most transition economies, modern HRM curricula have only been introduced in domestic universities in very recent years. Hence, almost all middle-aged and older employees working in personnel divisions or departments lack HRM expertise or have obsolete knowledge and experience acquired in a centrally planned economy (Tong, Straussman et al. 1999; Poon, Hung et al. 2009). Consequently, HR units' low status and limited competence have impeded the effective implementation of employee PM (Taylor 1992; Asim 2001; Liu and Dong 2012). For example, one study on the implementation of 
PM in Botswana indicates that HR professionals' insufficient knowledge caused the PM system to fail to achieve desirable goals (Dzimbiri 2008).

Fortunately, this does not mean that public organisations are unable to access updated HRM knowledge, particularly in the era of the internet and international integration. For example, in recent years, some public agencies in Vietnam have proactively organised study tours and sent their staff abroad to attend training programmes. Others have asked international donors such as the World Bank and UNDP to provide capacity-building projects. Meanwhile, other agencies can upgrade PM systems via self-study of material on the internet. These creative ways have helped these organisations gradually approach modern HRM practices and so have made renewal programmes more effective and viable.

Based on the above, the research formulates that:

Hypothesis 2: Agency accountability (H2a), HR autonomy (H2b), entrepreneurial leadership $(H 2 c)$ and $H R$ competence $(H 2 d)$ are associated with the development of employee PM practices.

Based on hypothesis 1 and hypothesis 2, the research continues to infer that the contextual factors (agency accountability, HR autonomy, entrepreneurial leadership and HR competence) can indirectly affect the outcomes of employee PM through the mediator of employee PM. In a study in English local governments, Walker, Damanpour et al. (2010) found that while management innovations did not directly affect organisational performance, they did indirectly influence organisational performance through the mediator of performance management. Accordingly, the research states that:

Hypothesis 3: Agency accountability (H3a), HR autonomy (H3b), entrepreneurial leadership $(\mathrm{H} 3 \mathrm{c})$ and $H R$ competence $(\mathrm{H} 3 \mathrm{~d})$ indirectly affect employee motivation, organisational commitment, job satisfaction and organisational performance through the mediation role of employee PM.

\subsubsection{Organisational culture factors}

Several researchers have indicated that organisational culture profoundly influences all aspects of management behaviours, including employee PM activities (Berrell, Wright et al. 1999; Aycan 2005; Ohemeng 2009). However, we have not sufficiently understood the way cultural norms affect appraisal decisions and other employee PM practices (DeNisi and Murphy 2017). 
To date, although there is not a stable definition of organisational culture, researchers tend to view it as the work-related values, ideologies, philosophies, beliefs, assumptions, expectations and attitudes that are shared among members of one organisation and characterised by particular organisations (Hofstede 1984; Furnham and Gunter 1993; Behery and Paton 2008). Organisational culture is formed from a number of different factors such as national culture, previous events of the organisation, work setting and personalities in the organisation (Mahler 1997). Koubek and Brewster (1995) argue that it is very difficult to design an appraisal system that can be appropriately used in different cultures. Indeed, different organisations have different cultures which in turn have different effects on employee behaviours and organisational effectiveness (Hofstede 1984; Schein 1985). Ohemeng (2009) affirms that culture and other traditional values have been viewed as one of the fundamental problems that have impeded and continue to impede the success of PM systems in developing countries.

Although a number of studies have investigated the association between national cultures, including uncertainty avoidance, power distance, collectivism vs. individualism, and employee PM (Mendonca and Kanungo 1996; Hofstede 1998; Lindholm 1999; Aycan 2005; Garnett, Marlowe et al. 2008; Chiang and Birtch 2010), the present research focuses on organisational culture for two reasons. First, national culture influences HRM practices through the mediation role of organisational culture (Van Muijen and Koopman 1994; Aycan, Kanungo et al. 1999). Second, unlike national culture, which is relatively stable, organisational culture can be changed. For example, a new chief executive's orientation can change the rules of the game, and as a result, new practices, habits, conventions and culture could be developed (Hofstede, Neuijen et al. 1990; Van Muijen and Koopman 1994).

Currently, the studies on the effects of organisational culture on employee PM often use the cultural dimensions of Hofstede (1998) such as process-oriented versus resultsoriented cultures; job-oriented versus employee-oriented cultures; professional versus parochial cultures; open system versus closed system cultures; and tightly versus loosely controlled cultures. They, though important, particularly in comparing the effects of western culture and eastern culture, may not capture cultural diversity in developing countries. Therefore, this research extends our understanding by investigating the effect of two other common cultural dimensions on the development of employee PM, namely nepotism and interpersonal relationships. 


\subsubsection{Nepotism}

The concept of "nepotism" stems from the Latin word "nepot" (nephew) and is often used to indicate the employment of relatives in the same organisation (Ford and McLaughlin 1986). Abdalla, Maghrabi et al. (1998) define nepotism as "the employment of relatives in the same organisation or the use of family influence to employ them in other organisations" (p.2). In fact, nepotism is likely to arise when employees are recruited, rewarded, promoted and favoured because of their kinship rather than their merit. Although in family-run businesses, nepotism might be regarded as an effective way to recruit and retain relatively cheap and loyal employees, in workplaces in general, nepotism is most often regarded as unprofessional behaviour and has a negative effect on employees, management and organisational performance (Bute 2011; Vveinhardt and Petrauskaite 2013).

The literature suggests that nepotism can affect employee PM practices in several ways. First, given the fear of displeasing subordinates who are related to senior leaders, supervisors often give preferential treatment in job assignment, appraisal, reward, and promotion to nepots regardless of whether or not these people have the necessary qualifications and work motivation to fulfil the work. For example, a study conducted in Lithuania indicated that the supervisors felt it was very difficult to evaluate the relatives of senior managers, even though they did not accomplish their duties (Vveinhardt and Petrauskaite 2013). Similarly, if one employee (nepotee) has to compete with a nepot for promotion, the likelihood of success will be very low (Hayajenh, Maghrabi et al. 1994), thereby leading to dissatisfaction, less motivation and commitment to the organisation (Hayajneh, Dwairi et al. 1994).

Second, nepotism undermines the performance of not only nepotees but also nepots. Supervisors tend to avoid assigning them challenging tasks and providing them with honest feedback and accurate ratings, so that nepots do not know their weaknesses and subsequently the need for improvement. This harms nepots because they do not know if a reward or promotion is due to their competence or kinship (Ichniowski 1988; Hayajneh, Dwairi et al. 1994).

Third, in a workplace where nepotism is present, the integrity of personnel policies is undermined (McCourt and Ramgutty-Wong 2003). For example, if a nepot is exempted from corrective measures for poor performance, it will be difficult to apply these measures to other poor performers. Most research concurs that nepotism has negative associations 
with job satisfaction, employee commitment, cooperation, production and performance (Hayajneh, Dwairi et al. 1994; Scoppa 2009; Vveinhardt and Petrauskaite 2013).

Although nepotism is a global phenomenon, it seems to be more prevalent and serious in transitional nations (Hayajneh, Dwairi et al. 1994; Arasli and Tumer 2008; Kragh 2012) as well as Vietnam (Poon, Hung et al. 2009). In these nations, inherent features such as the importance of family ties, weak implementation of laws or "the rule by man rather than rule by law", poor accountability and secretive personnel practices make nepotism and patronage very common (Tong, Straussman et al. 1999; Randma-Liiv 2005; Berman 2015; Rowshan, Ghasemnezhad et al. 2015). To control nepotism in the public sector, many initiatives have been proposed. Examples include:

(i) making personnel practices transparent and public such as introducing competitive examinations in recruiting new staff or promoting managerial personnel (Kragh 2012; Poocharoen and Brillantes 2013);

(ii) Inhibiting people in leadership positions from supervising their close relatives (Tong, Straussman et al. 1999) ;

(iii) Rotating senior leaders from their hometown to constrain family ties that breed nepotism (Tong, Straussman et al. 1999);

(iv) Introducing a regime of autonomy and self-responsibility for the use of administrative management payrolls and funds in order to enhance the accountability of chief executives in employing and managing employees (MOHA 2011).

\subsubsection{Interpersonal relationship culture}

The literature has come up with various definitions of "interpersonal relationships" because of its multi-dimensional nature, as well as different research purposes. In this research, the term "interpersonal relationships" is understood as personal ties that employees develop with their superior managers through social interactions both inside and outside working hours aimed at gaining favours in the workplace (Chen, Friedman et al. 2009; Cheung, Wu et al. 2009; Gu 2013). Unlike the relationship in leader-member exchange theory usually used in the western context, the personal relationship is often built on social occasions, such as home visiting, dining and gift giving (Cheung, Wu et al. 2009; Gu 2013). The integration of work and social relationships is very prevalent in Asian nations (Osigweh, Yg et al. 1993). Such interpersonal relationships are even stronger in societies influenced by 
Confucianism such as China and Vietnam (Law, Wong et al. 2000; Stanton and Pham 2014). In such contexts, interpersonal relationships are often given more weight than laws, regulations and merit (Gabrenya Jr and Hwang 1996; Luo 1997). In a study on appraisal in China, $\mathrm{Gu}$ (2013) found that interpersonal relationships are more dominant in workplaces characterised by high power distance and collectivism. In the Chinese public sector, although talent and political quality are often emphasised, what really counts for one's advancement is interpersonal relationships (Quanxi) with supervisors (Huang 2006). These phenomena are also very prevalent in Vietnam as a neighbouring country sharing many cultural values and political institutions (Acuña-Alfaro and Tran 2016).

Although the nature of interpersonal relationships might not be identical to nepotism, they influence the employee PM system in similar ways. In workplaces characterised by a high relationship culture, supervisors tend to divide their employees based on personal relationships and then offer different promotion opportunities and bonuses according to the degrees of intimacy of the relationships (Law, Wong et al. 2000; Cheng, Farh et al. 2002; Ouyang 2011). In such a context, HRM practices such as appraisal, reward and discipline struggle to function fairly and effectively (Bozionelos and Wang 2007; De Waal 2007; Bute 2011). In organisations with a strong relationship culture, supervisors have to consider the interpersonal relationships between subordinates and higher managers during the process of appraising, providing feedback, rewarding and addressing poor performance if they do not want to offend the senior managers and incur negative consequences (Bozionelos and Wang 2007). Consequently, this adversely affects other employees' perceived justice, trust in managers, commitment, motivation and satisfaction (Chen, Chen et al. 2004; Chen, Friedman et al. 2009; Gu 2013).

Although interpersonal relationships might be beneficial for both the supervisor and the subordinate within such relationships, they obviously pose a loss for third parties and the entire organisation (Van Buren and Leana 2000; Ma, Tang et al. 2015). In order to curb its negative effects on employee PM practice, some suggestions have been made. For example, governments and organisations should make personnel practices such as promotion and rewards transparent and merit-based (Ma, Tang et al. 2015). Besides, supervisors should be held accountable for appraisal decisions and appraisal criteria should be specific and measurable. Organisations should also use multiple raters such as supervisors, peers and 
customers to moderate the overall effects of interpersonal relationships (Bozionelos and Wang 2007).

Based on these arguments, this research hypothesises that:

Hypothesis 4: The relationships between the contextual factors and employee PM are moderated by the cultural variables of nepotism ( $\mathrm{H} 4 \mathrm{a})$, and interpersonal relationships $(H 4 b)$.

\subsection{Intervention practices}

Along with the effect of the above contextual factors, the failure of employee PM schemes is quite regularly attributed to the absence of practices supporting the implementation process (Roberts 2003; Biron, Farndale et al. 2011; Lawler, Benson et al. 2012). Miller (2001) points out that 70 percent of change programmes failed because the employees were not well prepared for the change process. Two practices frequently recommended are communication and training for users (Longenecker and Goff 1992; Randma-Liiv 2005; Caruth and Humphreys 2008; Biron, Farndale et al. 2011). This research examines whether these two intervention practices can improve the effectiveness of employee PM by strengthening the effects of the contextual factors on the development of the employee PM practices in the developing setting.

\subsubsection{Organisational communication}

Organisational communication concerns how an organisation educates, explains and prepares employees for the change process (Lewis 1999). Several studies have supported the positive correlation between communication and the effectiveness of change initiatives (Miller, Johnson et al. 1994; Armenakis and Harris 2002; Lewis 2006). Arguably, organisational communication increases understanding of the urgency and logic of change (Kotter and Schlesinger 1979; Armenakis and Bedeian 1999; Yuksel 2013) and enables employees to perceive potential benefits from the proposed change (Armenakis and Harris 2002). It also encourages participation and feedback from employees, thereby helping senior managers understand barriers and obstacles to implementation (Gallivan 2001). Employees who regard communication as effective tend to support for change more (Nelissen and van Selm 2008). 
Although research on the effect of organisational communication on employee PM remains limited, based on the above discussion we can predict that organisational communication is likely to strengthen the development and effectiveness of employee PM. First, organisational communication can reinforce the acceptance and support of relevant parties, including employees and supervisors. This is very important because employees' resistance can make the best-designed PM systems fail (Longenecker and Goff 1992; Kong, Kim et al. 2013; Kim 2014). In fact, there are various reasons leading to resistance to the innovation process of employee PM such as fear of job loss (Hardwick and Winsor 2002); bad experiences with previous reforms (McAdam and Mcgeough 2000); perceived work pressure from the change process (Hansson, Backlund et al. 2003; Dzimbiri 2008); lack of understanding of the process (Shin, Kalinowski et al. 1998); unnecessary bureaucratic burden (Kim 2014); or simply vague benefits from the change (Shin, Kalinowski et al. 1998). Therefore, an effective communication strategy can mitigate fears, uncertainty and resistance, thereby reinforcing positive attitudes towards the innovation (Nadler 1997; Bordia, Hobman et al. 2004; Kong, Kim et al. 2013).

Second, the communication process helps employees and managers understand the importance and purpose of the innovation as well as the expectations of the organisation, thereby curbing rating errors (Murphy, Kellam et al. 1984), and negative effects of organisational culture (Garnett, Marlowe et al. 2008). These in turn increase rating accuracy (Ilgen and Feldman 1983) and employees' acceptance of appraisal consequences (Kong, Kim et al. 2013). These are very significant to strengthen a smooth operation in early stages when an employee PM system is most likely to be imperfect.

To date, the research on the effect of communication on employee PM is limited. Almost all of it is qualitative and conducted in developed countries (Cunha, Vieira et al. 2018; Ohemeng, Amoako-Asiedu et al. 2018). Very little research has investigated the factual effect of communication on the success of employee PM. This research narrows this gap by providing evidence about the moderating role of communication in the success of employee PM in developing contexts.

\subsubsection{Providing training for users}

Training has been viewed as an indispensable activity when introducing employee PM (Randma-Liiv 2005; Cheng, Dainty et al. 2006; Liu and Dong 2012). This is because employee PM is regarded as one of the most sophisticated public management practices 
(Randma-Liiv 2005). Poor PM knowledge and skills have been recognised as one of the leading causes of PM failures (Longenecker and Goff 1992; Stoskopf 2002; Roberts 2003; Dzimbiri 2008; Shahina and Sumod 2015). This issue is even more serious in developing and transitional countries where modern management practices have only recently been domesticated (Randma-Liiv 2005; Liu and Dong 2012; Ohemeng, Zakari et al. 2015; Ahenkan, Tenakwah et al. 2018). Apart from providing the knowledge needed for implementation, training supplements communication efforts to help employees better understand the context for change (Cheng, Dainty et al. 2006). Tung, Baird et al. (2011) posit that the provision of training signifies that the organisations are willing to invest necessary resources and seriously commit to the implementation of employee PM.

Wexley (1984) defines training as "a planned effort by an organisation to facilitate the learning of job-related behaviour" (p.519). Many studies such as Lawler and McDermott (2003) and Haines III and St-Onge (2012) have indicated a high correlation between the presence of training and the effectiveness of employee PM. Providing PM training not only helps supervisors and employees better understand relevant concepts and principles but also presents opportunities to practise the system (Emerson 2002). Lawler and McDermott (2003) suggest that for training to be effective, it should be provided for both supervisors and subordinates.

For supervisors, training equips them with the skills necessary to manage employee performance effectively such as setting goals, developing performance standards, supervising performance, conducting appraisal interviews, providing feedback, discussing developmental needs, calculating ratings and solving conflicts (Taylor and O'Driscoll 1993; Stoskopf 2002; Roberts 2003; Haines III and St-Onge 2012). For example, Davis (2015) asserts that for a PM system to be effective, supervisors must provide honest feedback to their subordinates, even if it is negative. This is usually difficult if supervisors are not trained.

Training helps supervisors understand the importance of employee PM (Liu and Dong 2012) and plays a substantial role in constraining intentional or unconscious errors (Woehr and Huffcutt 1994; Gorman and Rentsch 2009). It also improves the effectiveness of feedback (Haines III and St-Onge 2012; Kinicki, Jacobson et al. 2013). Greenberg (1986) and Reinke (2003) suggest that employees are more likely to trust their supervisors and support the PM system if they believe that their supervisors are competent and have a good 
understanding of their performance. Longenecker and Goff (1992) and Roberts (2003) believe that without equipping managers with specific skills, appraisals are a sure route to frustration and dissatisfaction. This is because when facing difficulties in an appraisal situation, managers who lack appraisal skills may doubt their capabilities and are more likely to reduce their efforts to collect systematic information, avoid conflict and provide lenient ratings (Wood and Marshall 2008; Shahina and Sumod 2015). Consequently, if employees lack confidence in the efficacy of the rater, the PM system will tend to break down (Glendinning 2002; Roberts 2003).

For subordinates, training reinforces their perception of the purpose and on-going process of the new system, as well as helping them overcome the fear of uncertainty to positively participate in the innovation process such as setting goals and developing performance standards (Hansson, Backlund et al. 2003; Cheng, Dainty et al. 2006). Through training courses, employees acquire skills necessary to adapt to the new systems, as well as understanding of the standards used to measure their performance (Hansson, Backlund et al. 2003; Anjum, Yasmeen et al. 2011). Similarly, Lawler, Benson et al. (2012) posit that training enables employees to understand their role and the rights they should expect in the implementation process in terms of fairness and accountability.

As a result, Lawler, Benson et al. (2012) found that the effectiveness of employee PM has strong and positive correlations with training for both supervisors and employees. The studies in OECD countries and the U.S found that training for both supervisors and employees contributes to the success of the introduction of employee PM in the public sector (Montoya and Graham 2008).

To date, although no shortage of research has pointed out the necessity of communication and training in the implementation of employee PM, most is theoretical and interview-based research, and conducted in the developed context. This research narrows the gap by empirically investigating whether communication and training are able to strengthen the success of employee PM in the developing context.

Based on these arguments, this research hypothesises:

Hypothesis 5: The relationships between the contextual factors and employee PM are moderated by communication (H5a), and training (H5b). 


\subsection{Conceptual framework}

Based on the literature review, research questions and hypotheses, a conceptual framework is developed as Figure 3-1 below.

Figure 3-1: Conceptual framework of the development of performance management

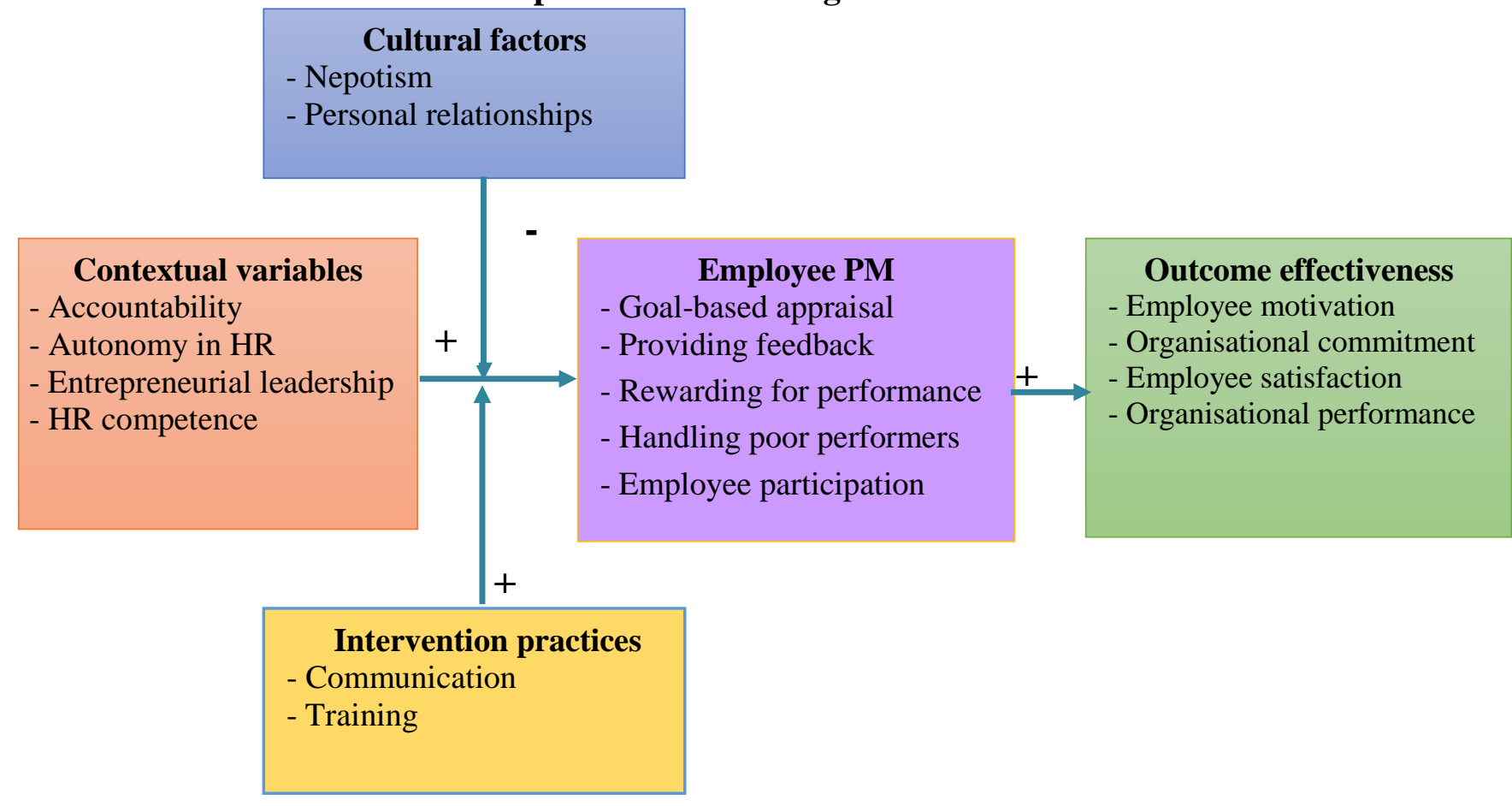

\subsection{Summary of Chapter 3}

Employee PM has become an increasing trend in the PAR process in transitional and developing countries aiming to meet the requirements of the transition process and achieve development goals. However, as well as universal technical issues, the introduction of employee PM in transitional countries is being hindered by particular contextual factors regarding constitutions, capacity and cultures. This has led to doubts about its applicability in these countries. This chapter reviews challenges as well as likely opportunities in introducing the five employee PM practices of goal-based appraisal, feedback, performancebased reward, addressing poor performers and employee participation. Based on this review, it hypothesises that the introduction of advanced PM practices in the developing context can lead to desired outcomes such as employee motivation, organisational commitment, job satisfaction and organisational performance. 
Additionally, in response to the call for learning about contextual factors in the introduction and implementation of employee PM, this chapter reviews the role of four contextual factors i.e. agency accountability, HR autonomy, entrepreneurial leadership, HR competence and two cultural variables i.e. nepotism and interpersonal relationships. The four factors are hypothesised as predicting factors for the advancement of employee PM. Meanwhile, the two cultural factors are hypothesised as moderators constraining the relationships above. Finally, the facilitating role of communication and training is reviewed. The research hypothesises that communication and training will moderate (strengthen) the effect of the contextual factors on the development of employee PM. All the arguments and hypotheses are then summarised in a conceptual framework shown in Figure 3-1. The next chapter presents the research methodology and design. 


\section{CHAPTER 4. RESEARCH METHODOLOGY}

This chapter presents the research methodology, which is defined as "the general approach the researcher takes in carrying out the research project" (Leedy and Ormrod 2005 p.12). First, it discusses the paradigm and research approach selected for the research. Second, it details the research design, as well as how data is collected, analysed and integrated. This is followed by a discussion of measures to ensure research quality and ethics. The final section summarises the chapter.

\subsection{The selection of paradigm and research approach}

A paradigm, or worldview, is a basic set of beliefs and assumption that guide research activities (Guba and Lincoln 2005). Creswell and Clark (2007) argue "all research needs a foundation for its inquiry and inquirers need to be aware of the implicit worldviews they bring to their studies" p.21. Although there are various categorisation of worldviews in the literature, Creswell (2014) suggest four main worldviews, namely the post-positivist, constructivist, transformative, and pragmatic worldview. Based on the research questions and purposes, and personal experiences, this research adopted the worldview of pragmatism.

The choice of the paradigm in this research came from the research philosophy of the researcher. As a policy-maker, the researcher believes that "Truth is what works at the time" (Creswell 2009 p.11). Rather than being adhered to any one system of philosophy, the author wanted to be flexible in epistemology, methods and procedures to investigate and understand the phenomena of employee PM sufficiently. For example, on the one hand, he wanted to use quantitative data to draw common rules about the implementation of PM in the developing context. On the other hand, he wanted to interact with participants to understand their perspectives about the implementation of employee PM. This helped the researcher to have a better understanding of the reasons behind phenomena and relationships. As a result, the combination of these two strands would be helpful to develop policies that can deal with practical issues in a given context comprehensively and effectively.

Unlike the post-positivist and constructivist worldview, the pragmatic worldview is not heavily dependent on epistemological perspectives. Instead, it focuses on the final consequence of the research or finds out "what works" and "solutions to problems"(Creswell and Clark 2007; Creswell 2014). Arguably, pragmatism is productive because it allows to adopt a useful middle stance philosophically and methodologically (Johnson and 
Onwuegbuzie 2004). Researchers adopting this worldview "are free to choose the methods, techniques, and procedures of research that best meet their needs and purposes" (Creswell 2014 p.11). Johnson and Onwuegbuzie (2004) posit that by attempting to "fit together the insights provided by qualitative and quantitative research into a workable solution... [pragmatism] offers the best opportunities for answering important research questions" p.16. As a result, pragmatism is often related to mixed methods research (Creswell and Clark 2007; Creswell 2014)

Mixed methods research is defined as "the type of research in which a researcher or team of researchers combines elements of qualitative and quantitative research approaches (e.g., use of qualitative and quantitative viewpoints, data collection, analysis, inference techniques) for the broad purposes of breadth and depth of understanding." (Johnson, Onwuegbuzie et al. 2007 p.123). In this vein, Habermas (1984) argues that "to achieve complete understanding of a social phenomenon, we need to apply multiple paradigm (or at least perspectives) to capture adequately (a) the objective (the material) world, (b) the subjective (my personal) world, and (c) the social (our inter-subjective) world" (Habermas, 1984, as cited in Ågerfalk (2013 p.252).

Depending on the way of combining elements of qualitative and quantitative research, there are various types of mixed methods strategies (Creswell 2014). Thus, it is useful for a mixed methods researcher to understand the nature, strengths and weaknesses of both qualitative and quantitative research to acquire high quality research results.

Qualitative research is advocated by constructivism that "claims that meaning does not exist in its own right; rather that it is constructed by human beings as they interact and engage in interpretation" (O'Leary 2004, p.10). Therefore, researchers should "rely as much as possible on the participants' views of the situation being studied" (Creswell 2009, p.8). Accordingly, qualitative research is defined as "an approach for exploring and understanding the meaning individuals or groups ascribe to a social or human problem. The process of research involves emerging questions and procedures, data typically collected in the participant's setting, data analysis inductively, building from particulars to general themes, and the research making interpretations of the meaning of the data" (Creswell 2009, p.4). Qualitative research often relates to the inductive approach (building theory) (Corbetta 2003) and is mostly conducted to understand 1) meanings, 2) surrounding contexts, 3) 
unanticipated phenomena, 4) processes, and 5) reasons behind causal relationships (Maxwell 2005).

On the other hand, quantitative research is supported by positivists and post-positivists who believe that knowledge can be acquired "based on careful observation and measurement of the objective reality that exists 'out there' in the world. Quantitative research is generally associated with the deductive approach (Corbetta 2003). Thus, developing numeric measures of observations and studying the behaviours of individuals becomes paramount." (Creswell 2009, p.7). Accordingly, quantitative research is regarded as "an approach for testing objective theories by examining the relationship among variables. These variables, in turn, can be measured, typically on instruments, so that numbered data can be analysed using statistical procedures." (Creswell 2009, p.4). The primary purpose of quantitative research is to predict trends, compare differences and examine the relationships between variables by analysing statistical data (Creswell 2005).

Mixed methods research assumes that all measurement is fallible, all observations are theory-laden, and people are inherently biased because of factors such as their cultural experience and worldview. Consequently, combining both quantitative and qualitative data enables a better understanding of what is happening in reality, particularly with complex problems (Trochim and Donnelly 2001; Creswell 2014). Mixed methods help capture the advantages of both quantitative and qualitative methods and overcome the inherent shortcomings of each individual method (O'Leary 2013).

Given the benefits of this combination, Malterud (2001) concludes that, "Rather than thinking of qualitative and quantitative strategies as incompatible, they should be seen as complementary" (p.483). For example, quantitative methods can predict trends, test the relationship among the investigated factors (Creswell 2005), and allow researchers to generalise findings to the targeted population. These are all useful for making policy (Easterby-Smith, Thorpe et al. 2012). Nevertheless, quantitative methods might not be effective in understanding processes or the intrinsic reasons prompting subjects' behaviour, thereby tending to be inflexible and artificial.

However, qualitative methods can offset these weaknesses. For example, information from interviews or focus groups allows us to understand the thinking and feeling of people, (Easterby-Smith, Thorpe et al. 2012) thereby having insight into their lived experiences and belief systems (O'Leary 2013). They also provide a detailed and in-depth examination of the 
issue under investigation (Creswell 2014). Silva and Wright (2008) argue that qualitative data is collected to "check and correct the quantitative data" and makes findings more robust.

Therefore, mixed methods research provides a more complete insight into the research phenomenon compared to each separate approach (Creswell 2014). In other words, the combination of quantitative and qualitative research can enhance the comprehensiveness and credibility of findings (Bryman 2015). As a result, the number of mixed methods publications and theses has rapidly increased in recent years (Haines 2011; Bryman 2015).

It is worth noting that, the mixed methods approach is not the best answer to every research problem. It requires broader skills and consumes more time and resources for data collection and analysis (Creswell and Clark 2007). Additionally, the use of this method is likely to create contradictory results that might not easily be consolidated (Easterby-Smith, Thorpe et al. 2012). Therefore, the mixed methods design can only be effective if research questions are well designed and implemented and comply with both qualitative and quantitative research standards (Bryman 2015).

As stated in the preceding chapter, the purpose of this study is to examine the hypotheses about the relationships between contextual factors and employee PM, as well as between employee PM and outcome variables such as employee motivation, commitment, job satisfaction and organisational performance. Simultaneously, it seeks to provide a better understanding of reasons behind causal relationships. This knowledge is important for policy-makers and practitioners to understand which factors are important, as well as how to make them more effective in reality. For these reasons, the mixed methods approach was identified as the most appropriate choice for this research.

\subsection{Research design}

Research design is defined as "plans and procedures for research that span the steps from broad assumptions to detailed methods of data collection, analysis and interpretation" (Creswell 2009, p.3). Depending on the priority for qualitative or quantitative research, and time sequence, the mixed methods approach can lead to different research designs. This study adopted the triangulation mixed methods design or the convergent parallel mixed methods design (convergent design) (Creswell 2009). This choice derived from the following reasons. 
First, the adoption of the convergent design aimed to minimize the dephasing between qualitative and quantitative strand caused by changes in each organisation such as the departure of chief executives and changes in personnel policies. As discussed above, the development of an employee PM system depends considerably on the determination and perspective of chief executives. The replacement of chief executives can make the entire PM system changed. Meanwhile, as a transition country, Vietnam is conducting several PAR programmes, including HRM policies. These could make the state of employee PM changed by the time. Therefore, collecting data in different times (as a sequential design) is likely to make the integration of results confounded.

Second, the convergent design used in this study allowed the researcher to shorten the data collection time (Iurato 2007), thereby ensuring that the research project would be accomplished on time. This is indeed important because the process of contacting and accessing participating organisations is usually time-consuming, particularly in the public sector in a socialist country where the information of public organisations is usually limited to the public. In addition, because almost all the constructs in this research were adopted from the Western context, they needed to be pre-tested to evaluate the compatibility before the official data collection. Time constraints meant a thorough sequential design was impossible, where the second phase of data collection would not have been able to begin until the data analysis of the first phase is completely accomplished.

Thirdly, as discussed by Creswell (2009), concurrent triangulation design can result in well validated and substantiated findings. A researcher first presents quantitative statistical results that are then followed by qualitative quotations that confirm or disconfirm the quantitative results.

In the convergent design, the researcher collects both qualitative and quantitative data concurrently, analyses it separately, and then merges the two. Accordingly, the convergent design involves collecting and analysing both quantitative and qualitative data during the same timeframe (Creswell and Clark 2007). The purpose of this design is to obtain different but complementary data aiming to provide a more thorough understanding and enhance the validity of the research findings (Creswell 2009). Arguably, this is particularly useful when a researcher wants to compare and contrast quantitative analysis results with qualitative analysis results (Creswell and Clark 2007). Specifically, in this research, the quantitative data was used to test hypotheses. Meanwhile, the main role of qualitative data was to 
triangulate quantitative analysis results, though it was also used to refine the questionnaire and sketch a more vivid picture about the research phenomenon.

Based on the flowchart of the basic procedures suggested by Creswell and Clark (2007), a research process is modified for this research as illustrated below.

Figure 4-1: Research process for the convergent parallel design

Adapted from Creswell and Clark (2007)

\begin{tabular}{|c|c|c|c|}
\hline No & Process & Quantitative & Qualitative \\
\hline 1. & $\begin{array}{l}\text { Data } \\
\text { collection }\end{array}$ & $\begin{array}{l}\text { - Identify population and sample } \\
\text { - Develop measures } \\
\text { - Conduct pilot tests } \\
\text { - Conduct survey }\end{array}$ & $\begin{array}{l}\text { - Select participants } \\
\text { - Develop interview questions } \\
\text { - Conduct interviews } \\
\text { - Collect secondary data }\end{array}$ \\
\hline 2. & Data analysis & $\begin{array}{l}\text { Process the quantitative data with } \\
\text { SPSS and AMOS }\end{array}$ & $\begin{array}{c}\text { Code the qualitative data according } \\
\text { to themes with Nvivo }\end{array}$ \\
\hline 3. & $\begin{array}{l}\text { Merging two } \\
\text { sets of results }\end{array}$ & \multicolumn{2}{|c|}{$\begin{array}{l}\text { - Compare, contrast and synthesise the results } \\
\text { - Identify convergence and differences between the } \\
\text { results }\end{array}$} \\
\hline 4. & Interpretation & \multicolumn{2}{|c|}{$\begin{array}{l}\text { - Summarize and interpret the separate results } \\
\text { - Discuss to what extent and in what ways results from the } \\
\text { two type of data converge, diverge, contradict or relate to } \\
\text { each other to produce a more complete understanding. }\end{array}$} \\
\hline
\end{tabular}

\subsection{Data collection}

Based on the research topic and research questions, this research targets organisations in the Vietnam public sector. Therefore, a diversity of public organisations was included in the research sample, including administration agencies, universities, research institutes and hospitals as principal employers of public employees. Although work characteristics, outputs and outcomes were likely different, they were all public organisations. In the current 
context in Vietnam, as influenced by the legacy of the Soviet management style, personnel policies for all public organisations, such as evaluation, salary, rewarding and promotions were almost the same. For example, the starting salary of graduates was the same regardless of the organisation they worked for such as an administration agency, a university or a research institute. The research acknowledges that while in many countries universities and hospitals have PM that is different from civil servants, that is not commonly the case in Vietnam.

Additionally, the diversity of participating organisations aimed to examine the effect of autonomy and other contextual factors in the innovation of employee PM. At the time of doing this research, universities, hospitals and research institutes were being granted more autonomy when they can create revenue from providing public services. In other words, the current state of employee PM in Vietnam is experimental in nature. The inclusion of employee PM reform organisations provides a varied range of efforts and conditions that allow us to assess the effects of employee PM on employee attitudes and organisational performance.

\subsubsection{Quantitative data collection}

The quantitative data in this research was collected in a field survey. Bhattacherjee (2012) defines a field survey as one non-experimental design that does not control independent variables or treatment, but measures these variables and tests their effects using statistical methods. The following discusses the process of the survey in detail.

\subsubsection{Population and sample identification}

The target population of this research is all employees officially working in the Vietnamese public sector. Up to the end of 2016, the total number of public employees in the country was $2,369,972$, excluding people working at the commune level ${ }^{1}$. These people were mostly working in public organisations under 30 ministries and ministry-level agencies, and 63 provinces nationwide. Of these, 272,952 people worked within administrative agencies whose tasks were making or enforcing policies. Meanwhile, $2,097,020$ people worked within 56,839 public service delivery institutions such as health, research, education institutions and so on.

\footnotetext{
${ }^{1}$ Website of Ministry of Home Affairs, accessed dated on 22/05/2017
} 
Because of the relatively large number of public organisations across the country, coupled with difficulties in accessing these organisations, the use of probability sampling seemed unfeasible, if not impossible, for this study. Additionally, as discussed in Chapter 2, the number of organisations having advanced employee PM systems is rather limited. Sampling is deliberately purposive in order to include organisations with different degrees of PM development. This research's purpose is to ensure a range of employee PM practices, rather than a representative sample of organisations or public servants. Because no list of such employee PM reform efforts exists in Vietnam, the research used diverse sources such as informant interviews, reports from the Ministry of Home Affairs, material from workshops and the network of HR professionals in order to canvass the range of employee PM efforts in the public sector. Based on our interviews and canvassing efforts, the sample could include with different development levels of employee PM such as advanced, transitional and laggard clusters. Although random sampling is generally preferable, nonrandom sampling is quite prominent in organisational studies, particularly when researchers conduct exploratory research to learn about the issues (Bryman 2008; Johnson 2010).

While acknowledging the stratified nature of the purposive sample, this research attempted to increase the representativeness of the sample. The participating organisations can represent diverse public organisations, including central vs local organisations; administration agencies vs service delivery institutions; large organisations with more than 800 employees vs relatively small organisations with only approximately 40 employees.

This research sampled 29 diverse public organisations within five central ministries and two provinces in Vietnam, including the Ministry of Agriculture and Rural Development, the Ministry of Natural Resources and Environment, the Ministry of Investment and Planning, the Ministry of Home Affairs, the State Bank of Vietnam, Danang province and Bacgiang province. As presented earlier, Danang and Bacgiang were permitted to implement pilot schemes regarding chief executive evaluation, output-based appraisal, online feedback and performance-based pay. Twelve organisations primarily do administration and regulatory work, whereas 17 organisations primarily provide services.

In each organisation, HR professionals were asked to provide a list of potential participants. To this end, we asked HR staff to provide a random selection of $10 \%$ of all permanent staff (employees and managers) who have at least three years' work experience in their unit, and stratified across different positions. In small departments, we asked HR 
staff to provide no fewer than 10 names. Based on these lists, 468 public employees were invited to participate in the survey. In total, 362 people returned questionnaires, giving a response rate of 77.35 percent. Of these, 322 questionnaires were fully completed and usable.

Based on common rules of thumb, this sample size is adequate for the research purpose. For example, Field (2009) suggests that a study needs at least 10-15 participants per variable. Gefen, Straub et al. (2000) suggest that the sample size should not be less than 10 times the number of items operationalising the most complex construct. For this research, examining hypotheses usually does not demand more than eight variables in a regression analysis and the most complex construct has no more than six items. So this sample size is large enough for quantitative analysis in this research.

In short, given the research purposes and conditions, this research adopted the purposive sampling technique. However, in an effort to increase representativeness, it obtained a relatively diverse sample across organisational functions, gender, age, qualification and job positions. The respondents tend to be well educated and senior. Most of them work in public service delivery institutions as these fields employ the largest number of employees in the public sector.

\subsubsection{Measurement development}

All constructs were measured by a seven-point Likert response scale from one (strongly disagree) to seven (strongly agree), except for HR autonomy which was measured by a five-point Likert scale. The survey questionnaire was initially developed in English and then translated into Vietnamese for collecting data. In order to ensure consistency and accuracy between the English and Vietnamese versions, an experienced translator translated the questionnaire back from Vietnamese to English. Almost all measures were developed based on existing measures, though some were modified to align with the research context. The following section illustrates how the constructs were formed.

Employee PM practices: As with the previous research e.g. Cho and Lee (2011) and Kinicki, Jacobson et al. (2013), this research views employee PM as a suite of component practices. Five PM practices, including goal-based appraisal, feedback, rewarding for performance, addressing poor performers and participation were included in this suite. Goalbased appraisal was measured by three items based on the prior works of Pooyan and Eberhardt (1989). A representative item of this measure is, "My evaluation is totally based 
on the work goals I have actually accomplished". Feedback was measured by three items adopted from a prior study of Kinicki, Jacobson et al. (2013). A representative item of this measure is, "My supervisor gives me timely feedback". Rewarding for performance was measured by three items adapted from Kim (2014). A representative item of this measure is, "My rewards reflect the effort I have put into my work". Employee participation was measured by two items adopted from Roberts and Reed (1996). A representative item of this measure is, "I can openly discuss job problems with my supervisor". Finally, one item concerned with addressing poor performers was developed by the author aiming to align with the research context. See Table 4-2 below.

Institution and capacity variables: Three institutional variables (accountability, HR autonomy and entrepreneurial leadership) and one capacity variable (HR competence) were examined in this research. Agency accountability was measured by six items modified from previous studies of Wang (2002) and Eun (2010) and Kim and Jung (2013). A representative item of this measure is, "My organisation's performance is seriously and carefully evaluated by upper agencies". HR autonomy was measured by six items adopted from Adamowki, Therriault et al. (2007). A representative item of this measure is, "What extent of autonomy does your organisation really have in recruitment practice". The word "really" was added to this question after preliminary interviews because there were sometimes differences between real autonomy and that stipulated in legal documents. Entrepreneurial leadership was measured by six items adopted from Renko, El Tarabishy et al. (2015). A representative item of this measure is, "My chief executive often comes up with radical improvement ideas to enhance my institution's operational effectiveness". HR competence was measured by six items modified from Han, Chou et al. (2006). A representative item of this measure is, "The HR professionals in our organisation have enough expertise to develop and organise an effective goal-based appraisal system"

Cultural factors: This research examines the moderation effect of two cultural factors, namely personal relationships and nepotism. Personal relationships were measured by five items adopted from Chen, Chen et al. (2004). A representative item of this measure is, "Supervisors find it difficult to be completely objective in performance appraisals because they have an interest in maintaining good relationships with their employees". Nepotism was measured by five items adopted from Abdalla, Maghrabi et al. (1998). A representative item of this measure is, "In my organisation, promotion is affected by kinship relationships". 
Intervention practices: To understand what can be done to strengthen the relationships between the contextual factors and PM practices, two intervention practices were investigated in this research, namely communication and training. Communication was measured by five items modified from Bouckenooghe, Devos et al. (2009). A representative item of this measure is, "We have been sufficiently informed on how the progress around the introduction of the performance management system is going". Meanwhile, training was measured by one self-developed item: "We received adequate training on employee performance management".

Outcome variables: Four dependent variables used to measure the effectiveness of employee PM practice are work motivation, organisational commitment, job satisfaction and organisational performance. Work motivation was measured by five items adapted from the prior works of Wright (2004) and Taylor (2007). A representative item is, "I put forth my best effort to get my job done regardless of the difficulties". Organisational commitment was measured by five items adopted from Meyer, Allen et al. (1993) and Rhoades, Eisenberger et al. (2001). A representative item of this measure is, "I feel a strong sense of belonging to my organisation". Job satisfaction was measured by a single-item scale adopted from Ting (1997) i.e. "In general, I am satisfied with my job". Although multi-item scales are more favourable for measurement, a single-item scale is increasingly prevalent in organisational research (Cantarelli, Belardinelli et al. 2016). Single-item scales are valid and useful not only for one dimensional but also for very complex constructs such as job satisfaction (Fuchs and Diamantopoulos 2009). Next, organisational performance was measured by five items adopted from Gibson and Birkinshaw (2004). A representative item of this measure is "This organisation is achieving its full potential". Although the use of perceived measures for organisational performance is regarded as a limitation, it is often employed in public sector research. Kearney, Hisrich et al. (2009) acknowledge that, "The challenge measuring performance in the public sector is great due to non-quantifiability, multicausality and perspective differences" (p.38). Some research indicates that employee perception is strongly related to objective measures (Boyd, Dess et al. 1993; Yang and Pandey 2009). Table 4-1 presents specific items used to measure the constructs as well as the reference resources. 
Table 4-1: Measures of variables

\begin{tabular}{|c|c|c|}
\hline Variable name & Survey items & Reference sources \\
\hline \multicolumn{3}{|c|}{ Employee PM practices } \\
\hline \multirow[t]{3}{*}{$\begin{array}{l}\text { Goal-based } \\
\text { appraisal }\end{array}$} & $\begin{array}{l}\text { My evaluation is totally based on the work goals I have } \\
\text { actually accomplished. }\end{array}$ & \multirow[t]{3}{*}{$\begin{array}{c}\text { Pooyan and } \\
\text { Eberhardt (1989) }\end{array}$} \\
\hline & My evaluation is based on my skills and abilities. & \\
\hline & $\begin{array}{l}\text { All my performance appraisal criteria are measurable and } \\
\text { objective. }\end{array}$ & \\
\hline \multirow[t]{3}{*}{ Feedback } & My supervisor gives me timely feedback. & \multirow{3}{*}{$\begin{array}{l}\text { Kinicki, Jacobson et } \\
\text { al. (2013) }\end{array}$} \\
\hline & $\begin{array}{l}\text { My supervisor gives me specific feedback about what is } \\
\text { good and bad about my performance. }\end{array}$ & \\
\hline & My supervisor gives me honest feedback. & \\
\hline \multirow[t]{3}{*}{$\begin{array}{l}\text { Rewarding for } \\
\text { performance }\end{array}$} & $\begin{array}{l}\text { There is a close tie between appraisal results and pay (extra } \\
\text { pay). }\end{array}$ & \multirow[t]{3}{*}{$\operatorname{Kim}(2014)$} \\
\hline & There is a close tie between appraisal results and rewards. & \\
\hline & My rewards reflect the effort I have put into my work. & \\
\hline $\begin{array}{l}\text { Addressing poor } \\
\text { performers }\end{array}$ & $\begin{array}{l}\text { In my organisation, those who do not accomplish their tasks } \\
\text { will be transferred to other jobs. }\end{array}$ & Self-developed \\
\hline \multirow[t]{2}{*}{ Participation } & I can openly discuss job problems with my supervisor. & \multirow{2}{*}{$\begin{array}{l}\text { Roberts and Reed } \\
\text { (1996) and Saad } \\
\text { (2014) }\end{array}$} \\
\hline & $\begin{array}{l}\text { I feel comfortable in expressing my opinion about the } \\
\text { rating result to the supervisor if it is unreasonable. }\end{array}$ & \\
\hline \multicolumn{3}{|l|}{ Contextual factors } \\
\hline \multirow[t]{6}{*}{ Accountability } & $\begin{array}{l}\text { Annually, my organisation must register key assignments } \\
\text { (goals) for approval from upper agencies. }\end{array}$ & \multirow{6}{*}{$\begin{array}{l}\text { Kim and Jung } \\
\text { (2013), Wang } \\
\text { (2002) and Eun } \\
\text { (2010) }\end{array}$} \\
\hline & $\begin{array}{l}\text { My organisation's performance is evaluated by agreed } \\
\text { specific indicators. }\end{array}$ & \\
\hline & $\begin{array}{l}\text { My chief executive receives proper recognition and } \\
\text { rewards corresponding to excellent performance. }\end{array}$ & \\
\hline & $\begin{array}{l}\text { My organisation's performance is rated and compared with } \\
\text { other institutions. }\end{array}$ & \\
\hline & $\begin{array}{l}\text { My organisation's performance is seriously and thoroughly } \\
\text { evaluated by upper agencies. }\end{array}$ & \\
\hline & $\begin{array}{l}\text { My chief executive receives criticism when the } \\
\text { organisation does not achieve the agreed goals. }\end{array}$ & \\
\hline \multirow[t]{6}{*}{ Autonomy in $H R$} & $\begin{array}{l}\text { What extent of autonomy does your organisation really } \\
\text { have in... } \\
\text { recruitment practice }\end{array}$ & \multirow[t]{6}{*}{$\begin{array}{l}\text { Adamowki, } \\
\text { Therriault et al. } \\
\text { (2007) }\end{array}$} \\
\hline & determining pay or bonus amounts & \\
\hline & promotion practice & \\
\hline & placing and assigning staff & \\
\hline & transferring unsuitable staff & \\
\hline & discharging unsuitable staff & \\
\hline \multirow[t]{3}{*}{$\begin{array}{l}\text { Entrepreneurial } \\
\text { leadership }\end{array}$} & $\begin{array}{l}\text { The chief executive of my organisation... } \\
\text { often comes up with radical improvement ideas to enhance } \\
\text { my institution's operational effectiveness }\end{array}$ & \multirow[t]{3}{*}{$\begin{array}{l}\text { Renko, El } \\
\text { Tarabishy et al. } \\
\quad(2015)\end{array}$} \\
\hline & is willing to take risks in his decisions & \\
\hline & demonstrates a passion for his/her work & \\
\hline
\end{tabular}




\begin{tabular}{|c|c|c|}
\hline & has a vision of the future of our organisation & \\
\hline & challenges and pushes us to act in a more innovative way & \\
\hline & is patient in pursuing new improvements and solutions & \\
\hline \multirow[t]{6}{*}{ HR competence } & $\begin{array}{l}\text { The HR professionals in our organisation... } \\
\text { have enough expertise to develop and organise an effective } \\
\text { goal-based appraisal system. }\end{array}$ & \multirow[t]{6}{*}{$\begin{array}{l}\text { Han, Chou et al. } \\
\text { (2006) }\end{array}$} \\
\hline & can develop clear and specific performance standards. & \\
\hline & $\begin{array}{l}\text { are competent to develop performance appraisal systems } \\
\text { that differentiate between employee performance levels } \\
\text { fairly and impartially. }\end{array}$ & \\
\hline & $\begin{array}{l}\text { are competent to develop a sound pay-for-performance } \\
\text { system. }\end{array}$ & \\
\hline & $\begin{array}{l}\text { can provide line managers with valuable insights or useful } \\
\text { advice regarding personnel management. }\end{array}$ & \\
\hline & have strong HR field expertise. & \\
\hline \multicolumn{3}{|l|}{ Cultural variables } \\
\hline \multirow[t]{5}{*}{$\begin{array}{l}\text { Interpersonal } \\
\text { relationship } \\
\quad \text { culture }\end{array}$} & $\begin{array}{l}\text { In my workplace, }, . \\
\text { a number of people joined my institution through personal } \\
\text { relationships. }\end{array}$ & \multirow[t]{5}{*}{$\begin{array}{l}\text { Chen, Chen et al. } \\
\text { (2004) }\end{array}$} \\
\hline & $\begin{array}{l}\text { those who have a good relationship with their supervisor } \\
\text { will get better treatment in jobs compared to others. }\end{array}$ & \\
\hline & $\begin{array}{l}\text { a person is more likely to be promoted when they have a } \\
\text { good relationship with the leader of the organisation. }\end{array}$ & \\
\hline & $\begin{array}{l}\text { supervisors find it difficult to be completely objective in } \\
\text { performance appraisals because they have an interest in } \\
\text { maintaining good relationships with their employees. }\end{array}$ & \\
\hline & $\begin{array}{l}\text { participating in interesting training with limited quota is } \\
\text { often affected by the interpersonal factor. }\end{array}$ & \\
\hline \multirow[t]{5}{*}{ Nepotism } & $\begin{array}{l}\text { In my organisation,... } \\
\text { supervisors are afraid of subordinates who are related to } \\
\text { high-level executives. }\end{array}$ & \multirow[t]{5}{*}{$\begin{array}{l}\text { Abdalla, Maghrabi } \\
\text { et al. (1998) and } \\
\text { Büte (2011) }\end{array}$} \\
\hline & promotion is affected by kinship relationships. & \\
\hline & $\begin{array}{l}\text { promotion or rewarding based on family ties has a negative } \\
\text { influence on employee motivation. }\end{array}$ & \\
\hline & $\begin{array}{l}\text { leaders' relatives are often promoted to good job positions, } \\
\text { though their competence is not as good as others. }\end{array}$ & \\
\hline & $\begin{array}{l}\text { Ability, knowledge and skill are less important than kinship } \\
\text { relationships in the promotion process. }\end{array}$ & \\
\hline \multicolumn{3}{|c|}{ Intervention practices } \\
\hline Communication & $\begin{array}{l}\text { My organisation has clearly explained the necessity of } \\
\text { introducing the performance management system. }\end{array}$ & $\begin{array}{l}\text { Bouckenooghe, } \\
\text { Devos et al. (2009) }\end{array}$ \\
\hline
\end{tabular}




\begin{tabular}{|c|c|c|}
\hline & $\begin{array}{l}\text { Our leaders have encouraged all of us to embrace the } \\
\text { performance management system. }\end{array}$ & \\
\hline & $\begin{array}{l}\text { We are sufficiently informed about progress around the } \\
\text { introduction of the performance management system. }\end{array}$ & \\
\hline & $\begin{array}{l}\text { Two-way communication between senior managers and } \\
\text { employees is very open and democratic. }\end{array}$ & \\
\hline & $\begin{array}{l}\text { Policies and procedures regarding the performance } \\
\text { management system are clearly communicated. }\end{array}$ & \\
\hline Training & $\begin{array}{l}\text { We have received adequate training on performance } \\
\text { management. }\end{array}$ & Self-developed \\
\hline Outcome variables & & \\
\hline $\begin{array}{l}\text { Employee } \\
\text { motivation }\end{array}$ & $\begin{array}{l}\text { I put forth my best effort to get my job done regardless of } \\
\text { the difficulties. }\end{array}$ & Wright (2004) \\
\hline & I am willing to start work early or stay late to finish a job. & \\
\hline & I do extra work for my job that isn't really expected of me. & \\
\hline & I emphasise doing my job right the first time. & \\
\hline & $\begin{array}{l}\text { I am motivated to put in as much effort into my job as I } \\
\text { possibly can. }\end{array}$ & \\
\hline Organisational & I feel a strong sense of belonging to my organisation. & Meyer, Allen et al. \\
\hline commitment & I feel personally attached to my work organisation. & $\begin{array}{l}\text { (1993) and } \\
\text { Rhoades, }\end{array}$ \\
\hline & $\begin{array}{l}\text { Working at my organisation has a great deal of personal } \\
\text { meaning for me. }\end{array}$ & $\begin{array}{l}\text { Eisenberger et al. } \\
\text { (2001) }\end{array}$ \\
\hline & I would be happy to work at my organisation until I retire. & \\
\hline & $\begin{array}{l}\text { I really feel that the problems faced by my organisation are } \\
\text { also my problems. }\end{array}$ & \\
\hline $\begin{array}{l}\text { Employee } \\
\text { satisfaction }\end{array}$ & In general, I am satisfied with my job. & Ting (1997) \\
\hline Organisational & This organisation is achieving its full potential. & Gibson and \\
\hline & $\begin{array}{l}\text { People at my level are satisfied with this organisation's } \\
\text { performance. }\end{array}$ & \\
\hline & $\begin{array}{l}\text { This organisation does a good job of satisfying its clients, } \\
\text { same-level agencies and upper-leaders. }\end{array}$ & \\
\hline & $\begin{array}{l}\text { This organisation gives me the opportunity and } \\
\text { encouragement to do the best work I am capable of. }\end{array}$ & \\
\hline & $\begin{array}{l}\text { The upper leaders and same-level agencies highly } \\
\text { appreciate our organisation's performance. }\end{array}$ & \\
\hline
\end{tabular}

\subsubsection{Pilot test}

Two pilot tests were conducted to consolidate the validity of the survey instruments and to improve the format, questions and scales (Creswell 2014). The first test was sent to 
10 public employees experienced in PM. Its primary purpose was to revise ambiguous questions and remove repetitions, as well as providing an initial evaluation of the internal consistency of the constructs. The researcher also asked the respondents to record the time they took to complete the questionnaire. Thanks to the first pilot test, some reverse coded items that confused the respondents and caused low internal reliability were amended. Additionally, in response to some respondents' feedback, the instruction was adjusted to emphasise that the researcher wanted to get the respondents' genuine perceptions of current PM practices, not their suggestions for improvement. The participants also reported that the questionnaire completion time ranged from 15 to 25 minutes, and most could complete it in 20 minutes. This time was reasonable to ensure an acceptable level of response quality and response rate.

After the questionnaire was refined based on the first pilot test, the second pilot test was carried out with the sample size $(n=42)$ from eight public organisations that varied in their development of employee PM. The second pilot test indicated that the measures in the questionnaire had acceptable levels of validity and reliability. Only minor changes were made before conducting the official survey.

\subsubsection{Survey}

Based on the initial information from the author's own experience, reports from ministries, and the mass media, the present research identified a list of participating organisations that had diverse contexts and employed PM practices. As an HR specialist working in one central ministry for approximately 15 years, the author had a network large enough to access these organisations. In the first meeting in each participating organisation, after introducing the self-identity, the author explained the study's goal, sampling criteria and measures necessary to protect the identity of participants. Once these were agreed by the chief executive, the HR unit would provide a list of possible participants who were permanent employees with at least three years' work experience. These people should hold different positions across units in each organisation.

Sampling in this research was mostly conducted via electronic forms (Qualtrics), though a small number of respondents used a paper form, as they were not accustomed to using online surveys. Online surveys have become more and more prominent because of their inherent advantages. They are economic in terms of cost and time, and allow researchers to easily access respondents from afar afield (Bryman 2008). Further, they 
enable respondents to voice opinions about sensitive topics (Wright 2005). With regard to the quality of responses, Gosling, Vazire et al. (2004) indicate that the quality of the data gathered by web-based studies is at least as good as that provided by traditional methods.

Based on the lists provided by HR staff, 468 employees from 29 public organisations were contacted and invited to participate in the survey. The deadline for completing the questionnaire was the $20^{\text {th }}$ working day after the questionnaire was sent. As well as seeking demographic and quantitative information, two open questions were included to ask if there were other influential factors and suggestions to improve the PM practice. The questionnaire is in Appendix A for the English version and Appendix B for the Vietnamese version. One reminder was sent 10 working days after it was first distributed.

According to their job titles, most respondents are policy-making officials, regulatory enforcers in administration agencies, researchers in institutes, medical staff in hospitals, and lecturers in universities. In Vietnam, institutes and universities operate under Ministries and their employees traditionally operate under very similar appraisal processes and standards as civil servants. Of the 322 respondents, 49.4 percent are male, 78.6 percent are working for organisations belonging to central ministries, 45.5 percent have less than 10 years' work experience, and 40.3 percent have 11-20 years' work experience. The majority of respondents (97.8 percent) hold a bachelor's degree or higher, and 36 percent hold managerial positions at divisional level or higher. The detailed demographic information is described in Table 4-2.

Table 4-2: Demographic profile of survey respondents

\begin{tabular}{|c|c|c|c|}
\hline Variable & Category & Frequency & Percentage \\
\hline Organisational level & $\begin{array}{ll}\text { - } & \text { Central } \\
\text { - } & \text { Provincial } \\
\end{array}$ & $\begin{array}{c}253 \\
69 \\
\end{array}$ & $\begin{array}{l}78.6 \% \\
21.4 \%\end{array}$ \\
\hline Organisational category & $\begin{array}{ll}\text { - } & \text { Administration } \\
\text { - } & \text { Service delivery }\end{array}$ & $\begin{array}{l}123 \\
199\end{array}$ & $\begin{array}{l}38.2 \% \\
61.8 \%\end{array}$ \\
\hline Gender & $\begin{array}{ll} & \text { Male } \\
\text { - } & \text { Female } \\
\end{array}$ & $\begin{array}{l}159 \\
163\end{array}$ & $\begin{array}{l}49.4 \% \\
50.6 \% \\
\end{array}$ \\
\hline Age & $\begin{array}{ll} & \text { Under } 30 \\
\text { - } & 30-\text { under } 40 \\
\text { - } & 40-50 \\
\text { - } & 50 \text { and over }\end{array}$ & $\begin{array}{c}52 \\
173 \\
78 \\
19\end{array}$ & $\begin{array}{c}16.1 \% \\
53.7 \% \\
24.2 \% \\
6.0 \%\end{array}$ \\
\hline Education & $\begin{array}{ll}\text { - } & \text { Doctor degree } \\
\text { - } & \text { Master degree } \\
\text { - } & \text { Bachelor degree } \\
\text { - } & \begin{array}{l}\text { Less than bachelor } \\
\text { degree }\end{array}\end{array}$ & $\begin{array}{c}25 \\
180 \\
110 \\
7\end{array}$ & $\begin{array}{c}7.8 \% \\
55.8 \% \\
34.2 \% \\
2.2 \%\end{array}$ \\
\hline
\end{tabular}




\begin{tabular}{|c|c|c|c|}
\hline Tenure & - From 25 and over & 23 & $7.1 \%$ \\
& - From 20 to under 25 & 23 & $7.1 \%$ \\
& - From 15 to under 20 & 50 & $15.5 \%$ \\
& - From 10 to under 15 & 80 & $24.8 \%$ \\
& - From 5 to under 10 & 95 & $29.5 \%$ \\
& - Less than 5 years & 51 & $16.0 \%$ \\
\hline Managerial position & - Departmental manager & 12 & $3.7 \%$ \\
& - Divisional manager & 104 & $32.3 \%$ \\
& - Non manager & 206 & $64.0 \%$ \\
\hline
\end{tabular}

\subsubsection{Qualitative data collection}

In this research, the qualitative data was mostly derived from the face-to-face interviews although it was supplemented with information from other sources such as the survey questionnaire (the open question section) and secondary data such as newspapers, legal documents, and reports from ministries.

\subsubsection{Interviews}

\section{Interview participants}

In the qualitative strand, maximal variation sampling was used. Seeking different views from individuals helps construct a holistic and dynamic picture of the research phenomena (Patton 2002). The sample size for qualitative research typically depends on the research questions, the scope of the qualitative study and the number of comparisons between groups in a study. The sample size can range from 1 or 2 people in a narrative study to 20 or 30 people in a grounded theory study (Creswell and Clark 2007). However, it is worth noting that the sample size should be able to induce convincing conclusions (Bryman 2015).

In this research, the interviewees were purposively selected so that they represented different groups in terms of gender, managerial position, organisational category, and particularly the maturity of the employee PM within their organisation. One advantage of the purposeful sampling is that it enables the researcher to access subjects who had specific experiences within different positions. The interviewees were permanent employees having at least 5 years' experience in the public sector. These people also participated in the survey except for some retired senior leaders.

In total, 30 interviews were conducted. As can be seen from Appendix C, the interview list included nine females (30 percent) and 21 males (70 percent). Of the interviewees, eight 
people (26.67 percent) are staff, nine people (30 percent) are HR managers, and 13 people (43.33 percent) are senior managers and leaders. Fifteen interviewees (50 percent) come from administration agencies while the remainder were from public service organisations such as health, education, and research institutions and other public service delivery organisations. About two-thirds of the interviewees (18 people) come from organisations under central agencies while one third is from provinces and localities.

\section{Interview procedure}

Most interviews are semi-structured. Semi-structured interviews are particularly useful in investigations related to respondents' experience (Marshall and Rossman 2006). Prior to starting each interview, the interviewees were informed about the identity of the researcher and the objective of the research as well as how their identity and the interview information would be protected. The interviewees were also advised to read and sign the consent form before being interviewed (see Appendix D). In general, the interviews took place in the interviewees' offices except for some which were conducted in other places suggested by the interviewees. On average, each interview lasted for two hours.

In order to create friendly rapport and motivate the interviewees to share information honestly, a small gift was given to each. The value of the gifts was not high, but it is a Vietnamese custom to manifest the researcher's appreciation for the information given by the informants. Victoria University of Wellington's Human Ethics Committee approved this gift giving.

Additionally, instead of using a recorder as normally suggested, the interviews were recorded with written notes. This is because many respondents seemed to be very reluctant to speak freely and honestly in front of a recorder though they were clearly informed that all information/material would be kept confidential. This problem has also been reported in some research conducted in China and Vietnam, which suggests that interviewees were afraid of unexpected consequences deriving from digital recording (Gu 2013; Phan 2014). To minimise data loss from note taking, the researcher tried to transcribe all the interviews into word documents straight after completing them.

\section{Interview questions}

Interview questions were developed to help the researcher understand why and how the employee PM practices can affect the outcome variables, as well as the reasons why the 
contextual factors and intervention practices can influence the development of employee PM in such a context. The questions prepared for each interview could be different depending on the position the informant held, including non-managerial employees, HR staff and senior executives. Accordingly, three different sets of questions were prepared to interview the three groups. See Appendix E (questions for non-managerial employees), Appendix F (questions for HR managers) and Appendix G (questions for senior managers).

\subsubsection{Other qualitative data}

Although qualitative data in this research was primarily collected via the interviews, the author also attempted to collect relevant secondary data. Lincoln and Guba (1985) posit that documents and other nonhuman resources can provide researchers with a rich source of information that represents the local language of the research context. Meanwhile, Creswell (2014) argues that these documents can be viewed as written evidence and help researchers to save time and the expense of transcribing. The secondary data in this research came from a variety of resources such as official documents from the government and the Communist Party of Vietnam, reports from ministries, mandates from public organisations regarding PM, newspapers, seminar reports, internet resources and news broadcasted on TV.

\subsection{Analytical strategy}

\subsubsection{Quantitative data analysis}

In this research, there were two units of analysis, including the individual level and the organisational level. The individual level was used for constructs such as PM practices (goalbased appraisal, feedback, reward-for-performance, addressing poor performers and employee participation), employee motivation, organisational commitment and job satisfaction. Whereas, the organisational level was applied to contextual variables, cultural variables, intervention variables as well as organisational performance. However, for convenience, all original data was collected through individuals' perceptions, though some conclusions drawn apply at the organisational level.

While acknowledging the limit of using individuals' perceptions for the organisational level, it was seemed is necessary because some information such as accountability, entrepreneurial leadership styles and organisational performance is currently not available at the organisational level. Therefore, this research used individuals' perceptions for such variables. Additionally, as can be seen in Table 5-12, respondents tended to agree with each 
other about the PM practices in their organisation. This indicates that the use of individuals' perceptions to make organisational level inferences is legitimate.

In fact, the use of individuals' perceptions to measure both the individual and the organisational construct is not uncommon e.g. Park (2014); Ryu (2010), Gibson and Birkinshaw (2004) and Bouckenooghe, Devos et al. (2009). In the article "Motivation of Public Managers as Raters in Performance Appraisal Developing a Model of Rater Motivation.",Park (2014) used individuals' perceptions to measure organisational culture and organisational policies (the organisational level) as well as self-perception of public service motivation and performance appraisal accuracy (the individual level).

All quantitative analysis in this study was conducted with the aid of SPSS statistics, version 23.0 and AMOS, version 25. Initially, factor analyses were conducted to test the validity and reliability of variables. Then, bivariate correlations were conducted to indicate the direction and strength of the relationships between the studied variables. Next, the hypotheses were examined in the following ways.

\subsubsection{Testing Hypothesis 1}

This hypothesis predicts that employee PM is associated with increased perceptions of employee motivation (H1a), organisational commitment (H1b), job satisfaction (H1c) and organisational performance (H1d).

Cluster analysis was first used to group all respondents based on the five PM practices discussed above. The goal of cluster analysis is to segment the respondents into clusters that have high homogeneity while ensuring high heterogeneity between clusters (Hair, Black et al. 2006). The first step was identifying the optimal number of clusters. As suggested by Arabie (1994), a hierarchical cluster was initially performed using Ward's method aimed at identifying the optimal number of clusters that is "the simplest structure possible that still represents homogeneous groupings" (Hair, Black et al. 2006 p.565). The result indicated that respondents could be classified into three groups corresponding to the laggard, transitional and advanced group. This was consistent with the initial intention of selecting organisations to participate in the study.

Then K-mean cluster analysis was performed for the clustering. The average scores of the PM practices were used to carry out the grouping (segmentation) process. Next, one-way Analysis of Variance ( $A N O V A$ ) was performed to examine the relationships of different PM development levels to the outcome variables including employee attitudes and 
organisational performance. If the average scores of each outcome variable are significantly different across the clusters, we can confidently confirm the hypothesis. In contrast, if they are not significantly different, we will reject the hypothesis.

\subsubsection{Testing Hypothesis 2}

Hypothesis 2 predicts that agency accountability (H2a), HR autonomy (H2b), entrepreneurial leadership $(\mathrm{H} 2 \mathrm{c})$ and $\mathrm{HR}$ competence $(\mathrm{H} 2 \mathrm{~d})$ are associated with the development of employee PM practices.

This hypothesis was examined by using a multinomial logistic regression. In this analysis, the independent variables were the contextual variables while the dependent variable was the cluster number gained from the cluster analysis. The cluster number was the extent to which an employee was classified according to his/her perception of the five PM practices being adopted within the organisation. Specifically, the higher the cluster number is, the more advanced (or developed) the employee PM system is. Accordingly, if the beta coefficient of one contextual variable in the multinomial logistic function is significantly different from zero, we can conclude that the contextual variable plays a significant role in the development of PM systems.

\subsubsection{Testing Hypothesis 3}

Based on the formulation of Hypothesis 1 and 2, Hypothesis 3 predicts that agency accountability (H3a), HR autonomy (H3b), entrepreneurial leadership (H3c) and HR competence (H3d) indirectly affect employee motivation, organisational commitment, job satisfaction and organisational performance through the mediation role of employee PM.

Observed variable path analysis in structural equation modelling (SEM) was used to test this hypothesis. SEM has become an increasingly popular choice for researchers in the social sciences (Chin 1998; Hooper, Coughlan et al. 2008). SEM is regarded as the second generation of multivariate analysis that allows researchers to carry out path analysis with the multi-level causal relationships between latent variables simultaneously (Chin 1998; Hair, Black et al. 2006). With regard to this research, SEM allows the researcher to examine indirect effects of the antecedents on the outcome variable through the mediator of employee PM. Furthermore, SEM can provide indices to evaluate the fit (goodness) of theoretical models with the collected data (Kelloway 1998). This is particularly helpful for this research 
which not only wants to examine indirect effects of the contextual variables on the outcomes but also whether the developed theoretical model is compatible with the collected data.

Currently, structural equation modelling can be performed by various software packages such as LISREL, EQS, RAMONA, CALIS, PLS and so on (Chin 1998). However, the Analysis of Moment Structures (AMOS), version 25, was used to test this hypothesis because of its strengths such as excellent graphical interface, well-organized output format and compatibility for specification research in the absence of theory and capability of creating more accurate estimates (Narayanan 2012). Once the theoretical model is examined using the collected data, by looking at indices in the output section, a researcher can intuitively assess indirect effects of the contextual factors, as well as how well the developed model fits with the collected data.

\subsubsection{Testing Hypothesis 4 and Hypothesis 5}

These two hypotheses involve testing the effect of moderation variables. Baron and Kenny (1986) define "a moderator as a qualitative (e.g. sex, race, class) or quantitative (e.g., level of reward) variable that affects the direction and/or strength of the relation between an independent or predictor variable and a dependent or criterion variable.” (p.1174)

Hypothesis 4 predicts that the relationships between the contextual factors and employee PM are moderated (weakened) by the cultural variables of nepotism (H4a), and interpersonal relationships (H4b). Meanwhile, Hypothesis 5 predicts that the relationships between the contextual factors and employee PM are moderated (strengthened) by communication (H5a), and training (H5b).

Accordingly, each cultural factor or intervention practice was considered in turn as a moderating variable on the relationship between the contextual variables (independent variables) and the development of employee PM practice (dependent variable). In which, the development of employee PM practice is computed or aggregated from the five component PM practices.

Zedeck (1971) suggested that the examination of the moderating effect should be conducted by regressing the three following equations.

$$
\begin{aligned}
& \text { 1.Y }=a+b^{*} X \\
& \text { 2.Y }=a+b^{*} X+c^{*} Z \\
& \text { 3.Y }=a+b^{*} X+c^{*} Z+d^{*} X^{*} Z
\end{aligned}
$$


In these equations, $\mathrm{Y}$ is the dependent variable; $\mathrm{X}$ is the independent variable; $\mathrm{Z}$ is the moderating variable. $\mathrm{ZX}$ is the interaction term achieved by multiplying the standardised independent variable with the standardised moderating variable.

Accordingly, the moderating effect is supported if the change in $\mathrm{R}^{2}$ (the coefficient of determination) between equation 3 and equation 2 as well as between equation 2 and equation 1 is significantly different (greater) from zero (Zedeck 1971; Cohen and Cohen 1975). As suggested by Kleinbaum, Kupper et al. (1988), the F value related to the change in $\mathrm{R}^{2}$ should be used for this test. Besides, a moderation hypothesis can be examined by testing whether the beta coefficients of independent and moderation variables in the first and second equation and the beta coefficient of interaction term in the third equation are significantly different from zero (Baron and Kenny 1986). However, the conclusions from the two methods should be the same.

Additionally, to address risks of multicollinearity (Cohen and Cohen 1975), the independent and moderator variables were standardised (centred) by subtracting the mean scores from the original score before performing regression. Then, the product terms were created by multiplying the corresponding standardised independent and moderating variables.

\subsubsection{Qualitative data analysis}

Rossman and Rallis (2003) contend "analysing and interpreting qualitative data is the process of deep immersion in the interview transcripts, field notes, and other materials you have collected; systematically organising these materials into salient themes and patterns, bringing meaning so the themes tell a coherent story" (p.270). More specifically, Taylor, Bogdan et al. (2015) suggest that qualitative data analysis is an ongoing process. It involves certain distinct activities, including (1) identifying themes, and developing concepts and propositions; (2) coding data and refining the understanding of the subject matter; (3) scrutinising the emerging themes and attempting to discount findings. Accordingly, developing themes and concepts is often conducted through reviewing literature, reading and rereading data, writing analytical memos and so on. Meanwhile, coding is regarded as a way of developing and refining interpretation of the data. In this vein, Creswell (2014) regards coding as taking text data or images obtained during data collection into categories or themes and then labelling these categories with terms (Creswell 2014). Finally, discounting data entails interpreting data in the context in which they are collected. 
Prior to collecting original data, relevant articles and secondary data collected from Vietnam, such as reports and mandates from Ministries and participating organisations, were coded. This process helped develop hypotheses, a conceptual framework, and subsequently pre-determined themes. Therefore, the main purpose of the interview data was to triangulate and expand results from quantitative data that was used to examine the hypotheses. As a result, qualitative data was collected and coded according to pre-determined themes. However, in this process, some new themes that were different from pre-determined themes but related to the research topic such as corruption, job types and side effects would be discussed.

Among different approaches to qualitative data analysis, template analysis is regarded as a pragmatic and practical approach because it allows researchers to be flexible to the needs of differing research projects (Brooks and King 2014). Template analysis refers to "the development of a coding template, which summarises themes identified by the researcher (s) as important in a data set and organises them in a meaningful and useful manner." (Brooks and King 2014 p.3). By adopting the analysis procedures suggested by these scholars, this research conducted qualitative data analysis as below:

\section{Step 1: Preliminary coding and initial template}

This step was conducted during the process of reviewing the literature and developing research hypotheses. Creswell (2014) suggests that for qualitative studies conducted in combination with the quantitative strand, researchers should initially develop a codebook or predetermined codes for coding the data. Data used in this period includes articles, books, reports from ministries, mandates from public organisations in Vietnam regarding employee performance appraisal and employee performance management. NVivo software were used to manage codes and themes under nodes. The initial template included following themes.

1. Employee PM practices

1.1 Aligning individual goals with organisational goals

1.2 Multiple feedback

1.3 Rewarding

1.4 Coaching

1.5 Employee participation 
1.6 Addressing poor performers

2. Contextual factors

2.1 Support from senior managers

2.2 Organisational culture

2.2.1 Process-oriented culture

2.2.2 Relationship-oriented culture

2.3 Autonomy

2.4 Accountability

2.5. Knowledge of HR staff

3. Convention practices

3.1 Communication

3.2 Training for uses

4. Effectiveness of employee PM

4.1 Employee motivation

4.2. Job satisfaction

4.3 Organisational commitment

4.4. Organisational performance

\section{Step 2: Developing and finalizing the template}

This step was carried out after collecting the preliminary interview data to evaluate the compatibility of hypotheses. Accordingly, interview transcripts and material collected in interviews were used for coding. The coding process was repeatedly done to ensure that all substantial sections of data related to the research questions were coded (Brooks, McCluskey et al. 2015). In the present research, all phrases or excerpts in the interviews related to the development and consequences of employee PM would be coded.

Arguably, the coding process enables the generation of themes or categories. Each theme displays multiple perspectives from the interviewees and is supported by quotations (Creswell 2014). Bryman (2015) suggests that a theme is what is formed on codes and relates 
to the research questions. It enables the researcher to have a theoretical understanding of the collected data.

It is advisable that "Where existing themes do not really 'fit' the new data, modification of the template may be necessary. New themes may be inserted and existing themes redefined or even deleted if they seem redundant". Thanks to this process, some themes such as coaching and process-oriented culture were dropped. Some themes were added, such as nepotism (the group of organisational culture) and communication (the group of intervention practices). Meanwhile, some themes were modified, for example: support from senior managers was replaced by entrepreneurial leadership and aligning individual goals with organisational goals was replaced by goal-based appraisal.

\section{Step 3: Refining and reporting the final template}

This step took place after all qualitative data had been collected. Once the coding process was completed, themes continued to be refined, connected, compared and consolidated in differing patterns to develop possible relationships, which in turn help to develop concepts and theories (Saldaña 2009). Arguably, the template analysis should enable to provide "the most valuable insights in the light of our research aims...[and] direct participant quotes serve to illustrate and support a researcher's interpretation of the raw data and allow readers to make their own assessment as to the credibility of the account presented." (Brooks and King 2014 p.8). Based on the final template as reported below (Figure 4-2), quotations are in turn presented according to themes as in Section 5.2 Qualitative analysis in Chapter 5. In which, employee PM practices are reported within the three clusters including: laggard, transitional and advanced cluster.

In addition to providing evidence to triangulate with the quantitative analysis, the qualitative analysis produced new themes such as corruption, job types and unwanted outcomes. This enabled to illustrate a more dynamic picture about the implementation of employee PM in the developing context. 
Figure 4-2: Final template of qualitative data analysis

\begin{tabular}{|c|c|}
\hline 1. Employee PM practices & 3. Organisational culture \\
\hline 1.1 Goal-based appraisal & 3.1 Personal relationships \\
\hline 1.2 Feedback & 3.2 Nepotism \\
\hline 1.3 Performance-based reward & 3.3. Egalitarianism \\
\hline 1.4 Addressing poor performers & 4. Intervention practices \\
\hline 1.5 Employee participation & 4.1 Training for users \\
\hline 2. Contextual factor & 4.2 Communication \\
\hline 2.1 Agency Accountability & 5. Desired outcomes \\
\hline 2.2 HR autonomy & 5.1 Employee motivation \\
\hline 2.3 Entrepreneurial leadership & 5.2 Organisational commitment \\
\hline 2.4 HR competence & 5.3 Job satisfaction \\
\hline 2.5 Corruption & 5.4. Organisational performance \\
\hline \multirow[t]{3}{*}{ 2.6 Job types } & 6. Unwanted outcomes \\
\hline & 6.1 Conflict between employees \\
\hline & 6.2. Conflict with the public interest \\
\hline
\end{tabular}

\subsection{Integration and interpretation of results}

The results of quantitative and qualitative data analysis are integrated and interpreted at the end of the chapter of results (Chapter 5). Creswell and Tashakkori (2007) argue that rather than reporting the two "strands" of qualitative and quantitative research independently, the findings should be integrated, or connected in some way. Specifically, "This side by side integration is often seen in published mixed methods studies in which a discussion section first provides quantitative statistical results followed by qualitative quotes that support or disconfirm the quantitative results" (Creswell 2009, p.212). Accordingly, the thesis will in turn presents statistical findings and qualitative findings. Then, the findings from the qualitative and quantitative analysis are compared and contrasted (Creswell 2009). 
In addition, in the discussion section of the Chapter 6, these findings are linked to the existing literature to examine whether they are consistent with or contradict previous studies.

\subsection{Establishing research quality}

The quality of social research is commonly evaluated by two criteria, namely validity and reliability (Yin 2003; Bryman 2008). Reliability is concerned with "the question of whether the results of a study are repeatable" while validity is concerned with "the integrity of the conclusions that are generated from a piece of research.” (Bryman 2008 p31,32). According to Bryman (2008), the violation of these criteria might undermine the integrity of the conclusions drawn from that data. In other words, Roberts, Priest et al. (2006) assert that these criteria are seen as the indicators demonstrating the trustworthiness of the research findings. To enhance the quality, researchers should embrace strategies to minimise potential issues in data collection, analysis and interpretation that might undermine the drawn conclusions (Creswell and Clark 2007). In mixed methods research, researchers should pay attention not only to each specific type of qualitative and quantitative strand but also to the interpretation of the results.

\subsubsection{Establishing and testing reliability and validity in the quantitative strand}

In the quantitative strand, reliability and validity often refer to the quality of measuring instruments. The following sections present the ways adopted by the research to enhance reliability and validity.

\subsubsection{Establishing and testing reliability}

Reliability in the quantitative research strand means that a measure should consistently reflect the construct that it is measuring (Field 2009). Yin (2003) contends that the objective of reliability is to minimise the errors and biases in the research process. One important indicator for evaluating reliability is internal reliability that is applied to the research with multiple-indicator measures (Bryman 2015). Internal reliability "is an assessment of the degree of consistency between multiple measurements of a variable" (Hair, Black et al. 2006 p.125).

In order to increase internal reliability for the constructs, this study attempted to seek existing scales that have been demonstrated high internal reliability (equal to or higher than 0.7). Once the instrument was developed, two pilot tests were carried out to discover and refine the scales with low internal reliability prior to administering to the target participants. 
For testing internal consistency reliability, Cronbach's alpha is often used. It is generally said that a value of 0.7 or 0.8 is an acceptable level for Cronbach's alpha (Field 2009; Bryman 2015). Kline (1992) suggests that when dealing with psychological constructs, a value of 0.7 or even below can be acceptable. Hair, Sarstedt et al. (2012) argue that once all Cronbach's alpha values of latent variables are higher than the threshold of 0.6, the internal consistency reliability is guaranteed.

\subsubsection{Establishing and testing validity}

In the quantitative strand, validity usually implies measurement validity that "refers to the issue of whether an indicator (or set of indicators) that is devised to gauge a concept really measures that concept." (Bryman 2015 p.158). The literature has proposed several ways for testing validity such as face validity, concurrent validity, predictive validity, construct validity, multicultural validity, and criterion validity (Colton and Covert 2007; Bryman 2015). However, there is no standard procedure compatible for all research. Instead, the validity methods should be contingent on the research situation (Punch 2013).

To increase the validity of the constructs, as suggested by Colton and Covert (2007), the present research embraced a number of measures. First, the researcher tried to understand the essence of the studied variables in relation to the research questions and the hypotheses by thoroughly reviewing relevant theories in the literature and interviewing key stakeholders in advance (establishing construct validity). Next, these initial measures were discussed with academics, experienced HR professionals in Vietnam and potential respondents to ensure that the measures were valid and compatible with the study's setting (establishing face validity, multicultural validity). Finally, two pilot tests were conducted to estimate correlation coefficients between the hypothesised variables (establishing concurrent validity).

To evaluate the validity of the measures, the present research examined three kinds of validity, including convergent, discriminant and construct validity. Convergent validity is defined as the "extent to which indicators of a specific construct converge or share a high proportion of variance in common" (Hair, Black et al. 2006 p.669). Although convergent validity might be evaluated by several criteria, it is normally assessed by factor loadings and Average Variance Extracted (AVE). With regard to utilising factor loadings, a rule of thumb is that standardised loading estimates should be equal to or greater than 0.5 (Hair, Black et al. 2006 p.686), though the threshold of 0.4 can sometimes be accepted (Igbaria, Zinatelli et 
al. 1997). Additionally, convergent validity is also regarded as acceptable if the AVE is equal to or greater than 0.5 (Fornell and Larcker 1981).

Construct validity is defined as the "extent to which a set of measured variables actually represents the theoretical latent construct those variables are designed to measure" (Hair, Black et al. 2006 p.671). One common method used to test construct validity is confirmatory factor analysis (CFA) (Lu 2006). CFA is conducted to test or confirm if a theoretical measurement model is valid (Hair, Black et al. 2006). In this study, CFA was carried out with the Analysis of Moment Structures (Amos). Although CFA can be evaluated by several fit indices, this study used four of the most common criteria to evaluate the goodness of models, including measurement models. These criteria included the ratio of a chi-square statistic and the degree of freedom $\left(\chi_{2} / \mathrm{df}\right)$, the Comparative Fit Index (CFI), the Goodness of Fit Index (GFI) and the Root Mean Square Error of Approximation (RMSEA). The table below presents cut-off points or thresholds to evaluate the goodness of a model.

Table 4-3: Cut-off points for determining model fit

\begin{tabular}{|c|c|c|}
\hline Criteria & Cut-off points & References \\
\hline$\chi^{2 / \mathrm{df}}$ & $<=5$ & (Wheaton, Muthen et al. 1977) \\
\cline { 2 - 3 } & Or $<=3$ & (Byrne 2016) \\
\hline CFI & $>=0.95$ & (MacCallum, Browne et al. 1996) \\
\hline GFI & $>=0.9$ & $($ Hu and Bentler 1999) \\
\hline RMSEA & $<=0.08$ & \\
\hline
\end{tabular}

Finally, discriminant validity is defined as the "extent to which a construct is truly distinct from other constructs. Thus high discriminant validity provides evidence that a construct is unique and captures some phenomena other measures do not" (Hair, Black et al. 2006 p.687). Fornell and Larcker (1981) posit that the square root of Average Variance Extracted (AVE) in each variable is a good indicator to establish discriminant validity. Accordingly, discriminant validity will be satisfied if the square root of each AVE is larger than other correlation values among the latent variables (Fornell and Larcker 1981; Hair, Black et al. 2006). The square root of each AVE is depicted in Table 5-11.

\subsubsection{Establishing validity and reliability in the qualitative strand}

Validity and reliability in the qualitative strand are somewhat different compared to that of the quantitative strand (Creswell 2009). According to Creswell (2014) "Qualitative 
validity means that the researcher checks for the accuracy of the findings by employing certain procedures while qualitative reliability indicates that the researcher's approach is consistent across different researchers and different projects" (p.201). Similarly, Kirk and Miller (1986) suggest that validity in qualitative research entails the quality of collected data while reliability is concerned with the degree of consistency of collected data. The following will discuss the ways to establish qualitative validity and reliability.

\subsubsection{Establishing qualitative validity}

In the present research, several procedures to increase the validity or credibility of the qualitative findings were undertaken. First, the writer conducted interviews with different groups to triangulate the collected data, including chief executives, HR professional, employees and even policy-makers. These informants needed to have at least 5 years of work experience so that they have a relatively sufficient understanding of current employee PM practice in their organisation. Arguably, validity is consolidated if themes are established based on the convergence of several participants (Creswell 2014).

Second, the researcher tried to establish a rapport with the interviewees by introducing himself, getting approval from the senior managers, having prestigious people liaise for the interviews, as well as presenting measures to protect the interviewee's identity and provided information. These practices were designed to enable the interviewees to speak out honestly and candidly about their perceptions.

Third, all data, even data that was discrepant and opposite to initial propositions was analysed. Creswell (2014) suggests that, by presenting contradictory evidence, the findings become more realistic and valid.

Finally, once the research draft was completed, the researcher had an independent researcher who had an understanding of the research context to review and provide objective comments about different aspects of the entire project, particularly the compatibility between the research questions and the collected data.

\subsubsection{Establishing qualitative reliability}

Qualitative reliability is concerned with the question of whether the researcher's approach is consistent across different researchers and different projects (Gibbs 2008; Creswell 2014). Accordingly, this research adopted the following measures to enhance qualitative reliability. First, he developed an interview protocol that follows the necessary 
procedures for conducting interviews. Yin (2009) contends that "the protocol is a major way of increasing the reliability of case study research and is intended to guide the investigator in carrying out the data collection" (p.76). For example, prior to beginning an interview, the researcher briefly introduced his identity, clearly articulated the purpose of the research and all measures necessary to protect interviewees' identity. Second, a set of questions (Appendices E, F and $\mathrm{G}$ ) was prepared in advance to keep this research focused on the information needed for the research. Third, all the interviews were transcribed immediately after the completed work to minimise mistakes or omissions as result of note taking. Additionally, the transcripts were carefully checked to ensure that there were no mistakes in the transcription process.

\subsection{Ethical considerations}

This research closely complied with the ethical standards for doing research. First, approval for doing this research was gained from the Human Ethics Committee of Victoria University of Wellington prior to collecting data. Thus, the research process was conducted in compliance with the university's ethical principles.

Second, prior to administering the survey questionnaires, the researcher sought to obtain approval from the senior managers or HR managers in the participating organisations. Furthermore, all potential participants were contacted in advance and informed about the nature and aims of the research. If a participant could not or did not want to take part in the survey or interview for any reason, he/she was replaced by another person.

Third, for the survey, no personally identifying information was collected. Meanwhile, with regard to the interviews, necessary measures were implemented to ensure that interviewees' identities could not be connected to their responses in the future e.g. the identity of interviewees would be confidentially protected by using pseudonyms. All the interviewees were provided with an informed consent agreement, which stated the rights of interviewees, e.g. they were free not to answer certain questions and could stop the interview at their convenience.

Finally, all resources including survey and interview documents will be destroyed after the completion of the research. The database with full anonymity will be retained so that this valuable data can be used for research in the future. 


\subsection{Limitation of research methods}

Like many other studies, research methods used in this research have a number of limitations. First, given that the sample could not be randomly drawn from the population, findings may not sufficiently represent all public organisations in the Vietnamese public sector. However, as stated, generalisation is not the chief target of the present study. Instead, it investigates conditions related to the development of the PM practices that might be usefully applied in other settings. In other words, this may provide a sound base for analytical generalization. Additionally, although the sample was not randomly selected, the researcher tried to select participating organisations that represent main characteristics of public organisations such as central versus local agencies, administration versus service delivery institutions, as well as large-scale versus small-scale organisations.

Second, all independent and dependent variables were collected at the same time, and hence, the internal validity may be influenced. This is because the expected dependent variables might have influenced the independent variables rather than the reverse (Bhattacherjee 2012). However, the use of multinomial logistic regression and structural equation modelling to investigate the causal relationships between predictor variables and outcome variables can minimise this effect (Horgan and Muhlau 2003; Yi 2013).

Third, because of difficulties that sometimes made it impossible to collect objective data, this study uses subjective data based on personal judgement to evaluate the organisations' performance. This is likely to lead to personal bias deriving from social desirability and other causes. However, Dess and Robinson (1984) assert that when objective data is unable to be obtained, subjective data should be used. This may be acceptable because employee perception is strongly related to objective measures (Boyd, Dess et al. 1993; Yang and Pandey 2009). In the present research, this issue was minimised by clearly stating to participants that their honest and objective reflection was most important and all information would be strictly confidential and safely managed.

Finally, as Malterud (2001) asserts, in qualitative studies an investigator's perspective, position and background will have certain impacts on the investigation method, findings and conclusions drawn. The researcher's professional experiences and initial preconceptions may influence the process of investigation and interpretation. This is likely to cause biases in the conclusions. Although the effect of the researcher is not completely eliminated, by 
acknowledging its existence and developing proper strategies, this research can assure the reliability and quality of the research (Malterud 2001).

\subsection{Summary of Chapter 4}

Based on pragmatic worldview, this study adopted the mixed methods approach in order to provide more complete answers to the research questions. Accordingly, the data for analysis was collected from a survey with a sample size of 322, and 30 interviews across 29 diverse organisations in the Vietnam public sector. For the data to be valid and reliable, the present research embraced a wide range of measures such as seeking existing scales with high internal reliability, getting feedback from experts, conducting pilot tests, increasing the diversity of informants and establishing standard procedures.

With regard to quantitative data analysis, the main techniques included cluster analysis (Hypothesis 1), multinomial logistic regression (Hypothesis 2), structural equation modelling (Hypothesis 3) and multiple regression (Hypotheses 4 and 5). Meanwhile, for the qualitative analysis, the processes of identifying themes, coding data and scrutinising emerging themes were conducted with the aid of NVivo. Having conducted the quantitative and qualitative analysis independently, the findings continued to be integrated, compared and contrasted to obtain rigorous conclusions. The next chapter presents research results. 


\section{CHAPTER 5. RESULTS}

This chapter reports the results of the analysis of the collected data. First, in the quantitative strand, it presents the results of testing the validity and reliability of the measures before reporting the results of examining the five hypotheses. Second, it reports the findings from the qualitative analysis. Quotations cited from the interviews are presented according to themes regarding the research hypotheses. Third, it integrates the qualitative and quantitative findings in relation to the research questions. The final section is the chapter summary.

\subsection{Quantitative analysis}

\subsubsection{Testing validity and reliability}

For quantitative research using latent variables, testing constructs validity and reliability is often a necessary requirement before examining the hypotheses. Additionally, with regard to using structural equation modelling, Anderson and Gerbing (1988) suggest that the measurement properties of a model should be evaluated before testing the structural relationships between latent variables. In this research, as mentioned in the previous chapter, the multiple-indicator measures were assessed by internal consistency reliability, convergent validity, construct validity and discriminant validity. Techniques used for these tests include the factor analysis in SPSS and the CFA in AMOS. The following sections will in turn present the results of testing internal consistency reliability, convergent validity and construct validity before reporting the results of testing discriminant validity.

Testing reliability, convergent and construct validity of the employee PM practices

Table 5-1 presents the results of the factor analysis (i.e. principal component analysis with direct oblimin rotation) on the five employee PM practices of: goal-based appraisal, feedback, rewarding for performance, addressing poor performers and employee participation. All 12 items loaded on the five expected constructs have factor loadings greater than 0.5 , while only having small loadings on the other constructs. Also, the Average Variance Extracted (AVE) values of the respective constructs are higher than 0.5, the minimum acceptable level for convergent validity (Fornell and Larcker 1981). Cronbach's 
alpha values for all the PM practices, except addressing poor performers that only had one item, are greater than the benchmark of 0.7 . Therefore, convergent validity and internal reliability of the five employee PM practices are confirmed.

Table 5-1: Factor loading of the five employee PM practices

\begin{tabular}{|c|c|c|c|c|c|c|c|}
\hline \multirow[b]{2}{*}{$\mathbf{N}=322$} & \multicolumn{5}{|c|}{ Factors } & \multirow[t]{2}{*}{ Alpha } & \multirow[t]{2}{*}{ AVE } \\
\hline & 1 & 2 & 3 & 4 & 5 & & \\
\hline Goal-based appraisal 1 & .892 & .101 & -.072 & -.018 & -.082 & \multirow[t]{3}{*}{.908} & \multirow[t]{3}{*}{.749} \\
\hline Goal-based appraisal 2 & .953 & -.070 & .020 & .059 & .005 & & \\
\hline Goal-based appraisal 3 & .738 & .053 & -.017 & .037 & .175 & & \\
\hline Feedback 1 & .062 & .062 & -.914 & -.065 & -.012 & \multirow[t]{3}{*}{.937} & \multirow[t]{3}{*}{.844} \\
\hline Feedback 2 & .084 & -.016 & -.905 & -.013 & .021 & & \\
\hline Feedback 3 & -.107 & -.118 & -.938 & .103 & .025 & & \\
\hline Reward for performance 1 & -.039 & .890 & .021 & .004 & .116 & \multirow[t]{3}{*}{.925} & \multirow[t]{3}{*}{754} \\
\hline Reward for performance 2 & -.007 & .990 & -.005 & .017 & -.057 & & \\
\hline Reward for performance 3 & .165 & .700 & -.110 & .062 & .013 & & \\
\hline Addressing poor performers & .027 & .037 & -.037 & .019 & .932 & NA & NA \\
\hline Participation 1 & .022 & -.045 & -.032 & .955 & -.026 & \multirow[t]{2}{*}{.883} & \multirow[t]{2}{*}{.838} \\
\hline Participation 2 & .011 & .091 & .017 & .875 & .041 & & \\
\hline
\end{tabular}

Note:

- Factor loadings and Cronbach's alpha values were calculated using SPSS. The AVE values, as suggested by Hair, Black et al. (2006), were manually calculated using the formula: $\mathrm{AVE}=\frac{\sum_{i=1}^{n} L_{i}^{2}}{\mathrm{n}} ; \mathrm{Li}$ represents the standardised factor loading while $\mathrm{i}$ is the number of indicators (items).

- The actual items can be found in Table 4-2.

After convergent validity and internal reliability were evaluated, CFA was conducted with the aid of AMOS to evaluate construct validity. Among these five constructs, the construct of addressing poor performers has only one item (indicator) so its error variance needed to be fixed as to be one value. It has been suggested that the error variance should be calculated by multiplying the variance of the indicator by the difference of one and the reliability estimate (Petrescu 2013). If the value of the reliability estimate cannot be calculated, a conservative arbitrary value such as 0.85 should be used (Jöreskog and Sörbom 1982). 
In the initial model, the results of $\chi_{2} / \mathrm{df}, \mathrm{GFI}, \mathrm{CFI}$ meet their thresholds. However, the RMSEA value of 0.093 is a bit higher than the cut-off point of 0.08 . The modification indices indicated that there is a high covariance between the errors of indicators within the construct of rewarding. After fixing this issue, all the criteria are satisfied, meaning that construct validity is confirmed. Table 5-2 below illustrates the result of CFA over the employee PM practices before and after fixing the problem of covariance.

Table 5-2: Results of CFA for the employee PM practices

\begin{tabular}{|l|c|c|c|c|c|c|c|}
\hline Model fix index & $\chi \mathbf{2}$ & $\mathbf{D f}$ & $\chi \mathbf{2 / d f}$ & $\mathbf{P}_{\text {value }}$ & GFI & CFI & RMSEA \\
\hline Initial model & 173.953 & 46 & 3.782 & .000 & .914 & .964 & .093 \\
\hline $\begin{array}{l}\text { The model after } \\
\text { fixing covariance } \\
\text { of errors within } \\
\text { same construct }\end{array}$ & 120.671 & 44 & 2.743 & .000 & .939 & .979 & .074 \\
\hline
\end{tabular}

Testing reliability, convergent and construct validity of the contextual variables

Table 5-3 demonstrates that the constructs of the contextual variables have relatively good convergent validity and internal reliability. All the items have high factor loadings on the respective factors. Meanwhile, Cronbach's alpha and the AVE values are over the minimum thresholds of 0.7 and 0.5 respectively. The results from CFA depicted in Table 5.4 also confirm the construct validity when the model fit is good after fixing the covariance of errors within the same construct, although GFI is a bit lower than the cut-off point of 0.9.

Table 5-3: Factor loading of the contextual factors

\begin{tabular}{|c|c|c|c|c|c|c|}
\hline \multirow[b]{2}{*}{$\mathbf{N}=\mathbf{3 2 2}$} & \multicolumn{4}{|c|}{ Factors } & \multirow[t]{2}{*}{ Alpha } & \multirow[t]{2}{*}{ AVE } \\
\hline & 1 & 2 & 3 & 4 & & \\
\hline Accountability 1 & .190 & -.041 & .000 & .593 & \multirow[t]{6}{*}{0.890} & \multirow[t]{6}{*}{.583} \\
\hline Accountability 2 & .133 & -.005 & .000 & .781 & & \\
\hline Accountability 3 & .035 & .054 & -.001 & .772 & & \\
\hline Accountability 4 & -.124 & .079 & -.018 & .851 & & \\
\hline Accountability 5 & -.087 & .043 & -.163 & .814 & & \\
\hline Accountability 6 & .130 & -.066 & .060 & .745 & & \\
\hline HR autonomy 1 & -.113 & .729 & -.085 & .111 & \multirow[t]{2}{*}{0.899} & \multirow[t]{2}{*}{.650} \\
\hline HR autonomy 2 & .007 & .732 & -.051 & .003 & & \\
\hline
\end{tabular}




\begin{tabular}{|c|c|c|c|c|c|c|}
\hline HR autonomy 3 & .073 & .813 & -.094 & -.080 & & \\
\hline HR autonomy 4 & .019 & .865 & .099 & .040 & & \\
\hline HR autonomy 5 & .082 & .876 & .128 & -.028 & & \\
\hline HR autonomy 6 & .032 & .809 & -.054 & .003 & & \\
\hline Entrepreneurship 1 & .851 & .061 & -.088 & -.027 & \multirow[t]{6}{*}{0.929} & \multirow[t]{6}{*}{.641} \\
\hline Entrepreneurship 2 & .812 & .030 & .014 & .069 & & \\
\hline Entrepreneurship 3 & .816 & .048 & -.087 & .026 & & \\
\hline Entrepreneurship 4 & .778 & .078 & .066 & -.013 & & \\
\hline Entrepreneurship 5 & .750 & -.032 & -.074 & .078 & & \\
\hline Entrepreneurship 6 & .793 & -.068 & -.105 & .106 & & \\
\hline HR competence 1 & .073 & .003 & -.881 & -.044 & \multirow[t]{6}{*}{0.956} & \multirow[t]{6}{*}{.811} \\
\hline HR competence 2 & .003 & .014 & -.934 & .000 & & \\
\hline HR competence 3 & -.023 & -.010 & -.930 & .069 & & \\
\hline HR competence 4 & -.074 & .031 & -.881 & .108 & & \\
\hline HR competence 5 & .070 & .009 & -.897 & -.035 & & \\
\hline HR competence 6 & .101 & .002 & -.880 & -.024 & & \\
\hline
\end{tabular}

Table 5-4: Results of CFA of the contextual factors

\begin{tabular}{|l|c|c|c|c|c|c|c|}
\hline Model fix index & $\chi_{2}$ & $\mathbf{d f}$ & $\chi_{2} / \mathbf{d f}$ & $\mathbf{P}_{\text {value }}$ & GFI & CFI & RMSEA \\
\hline Initial model & 818.577 & 246 & 3.328 & .000 & .818 & .916 & .085 \\
\hline $\begin{array}{l}\text { The model after fixing } \\
\text { covariance of errors } \\
\text { within the same } \\
\text { construct }\end{array}$ & 449.522 & 234 & 1.921 & .000 & .897 & .968 & .054 \\
\hline
\end{tabular}

\section{Testing reliability, convergent and construct validity of the cultural variables}

The results of testing reliability, convergent validity and construct validity for the two cultural variables are shown in Table 5-5 and Table 5-6. The two cultural variables of personal relationships and nepotism have AVE values greater than the acceptable level of 0.5 while all the indicators load on their respective constructs in accordance with the expectation. Simultaneously, internal reliability is also confirmed by the Cronbach's alpha values of 0.931 and 0.954 respectively. The results from CFA, as illustrated in Table 5-6, 
prove that construct validity is ensured when the goodness-of-fit statistics meet their cut-off points.

Table 5-5: Factor loading of the cultural factors

\begin{tabular}{|c|c|c|c|c|}
\hline \multirow[b]{2}{*}{$\mathbf{N}=322$} & \multicolumn{2}{|c|}{ Factors } & \multirow[t]{2}{*}{ Alpha } & \multirow[t]{2}{*}{ AVE } \\
\hline & 1 & 2 & & \\
\hline Personal Relationship 1 & .050 & .835 & \multirow[t]{5}{*}{0.931} & \multirow[t]{5}{*}{.706} \\
\hline Personal Relationship 2 & .101 & .849 & & \\
\hline Personal Relationship 3 & .026 & .922 & & \\
\hline Personal Relationship 4 & -.121 & .943 & & \\
\hline Personal Relationship 5 & .294 & .610 & & \\
\hline Nepotism 1 & .627 & .304 & \multirow[t]{5}{*}{0.954} & \multirow[t]{5}{*}{.766} \\
\hline Nepotism 2 & .815 & .159 & & \\
\hline Nepotism 3 & .939 & -.038 & & \\
\hline Nepotism 4 & .956 & -.008 & & \\
\hline Nepotism 5 & .988 & -.063 & & \\
\hline
\end{tabular}

Table 5-6: Results of CFA of the cultural factors

\begin{tabular}{|l|c|c|c|c|c|c|c|}
\hline Model fix index & $\boldsymbol{\chi 2}$ & $\mathbf{d f}$ & $\boldsymbol{\chi} \mathbf{2 / d f}$ & $\mathbf{P}_{\text {value }}$ & GFI & CFI & RMSEA \\
\hline Initial model & 126.371 & 26 & 4.860 & .000 & .921 & .969 & .110 \\
\hline $\begin{array}{l}\text { The model after fixing } \\
\text { covariance of errors } \\
\text { within the same } \\
\text { construct }\end{array}$ & 54.050 & 21 & 2.574 & .000 & .965 & .990 & .070 \\
\hline
\end{tabular}

Testing reliability, convergent validity and construct validity of the intervention variables

Table 5-7 displays the results of the factor analysis on the two intervention practices of communication and training. The initial test indicates that one item of the construct communication failed to load on the expected factor. This item was then dropped from the construct. The result indicated that all the indicators loaded on the respective factors with factor loadings greater than 0.5 . Though the internal reliability of the construct training is not applicable due to the one-indicator construct, Cronbach's alpha for communication is 0.946 . Hence, convergent validity and internal reliability are accepted. 
To conduct CFA, because the construct of training only has one item (indicator), the error variance was fixed at a constant value as discussed in the preceding section. As reported in Table 5-8, construct validity is guaranteed when the model fit indices are good.

Table 5-7: Factor loading of the intervention practices

\begin{tabular}{|l|c|l|l|l|}
\hline \multirow{2}{*}{\multicolumn{1}{|c|}{$\mathbf{N}=\mathbf{3 2 2}$}} & \multicolumn{2}{c|}{ Factors } & \multirow{2}{*}{ Alpha } & AVE \\
\cline { 2 - 3 } & $\mathbf{1}$ & $\mathbf{2}$ & & \\
\hline Communication 1 & $\mathbf{. 9 9 5}$ & -0.069 & 0.946 & .661 \\
\hline Communication 2 & $\mathbf{. 9 7 0}$ & -0.020 & & \\
\hline Communication 3 & $\mathbf{. 6 3 6}$ & .347 & & \\
\hline Communication 4 & $\mathbf{. 5 5 8}$ & .431 & & \\
\hline Training & .019 & $\mathbf{. 9 6 5}$ & NA & \\
\hline
\end{tabular}

Table 5-8: Results of CFA of the intervention factors

\begin{tabular}{|l|c|c|c|c|c|c|c|}
\hline Model fix index & $\chi \mathbf{2}$ & $\mathbf{d f}$ & $\chi \mathbf{2 / d f}$ & $\mathbf{P}_{\text {value }}$ & GFI & CFI & RMSEA \\
\hline Initial model & 61.610 & 9 & 6.846 & .000 & .937 & .974 & .135 \\
\hline $\begin{array}{l}\text { The model after fixing } \\
\text { covariance of errors } \\
\text { within the same } \\
\text { construct }\end{array}$ & 18 & 6 & 3 & .000 & .982 & .994 & .079 \\
\hline
\end{tabular}

\section{Testing reliability, convergent and construct validity of the outcome variables}

Finally, Table 5-9 and Table 5-10 show the results of assessing reliability, convergent validity and construct validity of the four outcome variables: work motivation, organisational commitment, job satisfaction and organisational performance. The initial test showed that two items of the work motivation construct and one item of the organisational commitment construct failed to load on the expected factors. These items were dropped one after the other. The result indicates that the convergent validity and internal reliability of these constructs are confirmed when Cronbach's alpha values and AVE coefficients are greater than 0.7 and 0.5 respectively. Similarly, the results of running CFA (Table 5-10) show that the construct validity is accepted when the indices of model fit are met after the error variance was fixed. 
Table 5-9: Factor loading of the outcome effectiveness variables

\begin{tabular}{|c|c|c|c|c|c|c|}
\hline \multirow[b]{2}{*}{$\mathbf{N}=322$} & \multicolumn{4}{|c|}{ Factors } & \multirow[t]{2}{*}{ Alpha } & \multirow[t]{2}{*}{ AVE } \\
\hline & 1 & 2 & 3 & 4 & & \\
\hline Work motivation 1 & .096 & .792 & -.078 & -.084 & \multirow[t]{3}{*}{.796} & \multirow[t]{3}{*}{.667} \\
\hline Work motivation 2 & .077 & .812 & .120 & .323 & & \\
\hline Work motivation 3 & -.067 & .845 & -.137 & -.195 & & \\
\hline Organisational commitment 1 & .137 & .044 & -.762 & -.006 & \multirow[t]{4}{*}{.987} & \multirow[t]{4}{*}{.647} \\
\hline Organisational commitment 2 & .083 & .064 & -.819 & -.194 & & \\
\hline Organisational commitment 3 & -.050 & .013 & -.919 & .102 & & \\
\hline Organisational commitment 4 & .006 & .131 & -.701 & .477 & & \\
\hline Job satisfaction & .166 & .014 & -.789 & -.121 & NA & NA \\
\hline Organisation performance 1 & .840 & .050 & -.041 & -.143 & \multirow[t]{5}{*}{.932} & \multirow[t]{5}{*}{.719} \\
\hline Organisation performance 2 & .976 & .040 & .127 & -.050 & & \\
\hline Organisation performance 3 & .917 & -.069 & -.028 & .199 & & \\
\hline Organisation performance 4 & .735 & .011 & -.208 & -.086 & & \\
\hline Organisation performance 5 & .744 & .066 & -.146 & .068 & & \\
\hline
\end{tabular}

Table 5-10: Results of CFA of the outcome effectiveness factors

\begin{tabular}{|c|c|c|c|c|c|c|c|}
\hline Model fix index & $\chi \mathbf{2}$ & $\mathbf{d f}$ & $\chi \mathbf{2 / d f}$ & $\mathbf{P}_{\text {value }}$ & GFI & CFI & RMSEA \\
\hline Initial model & 266.367 & 60 & 4.439 & .000 & .890 & .938 & .104 \\
\hline $\begin{array}{c}\text { The model after fixing } \\
\text { covariance of errors } \\
\text { within the same } \\
\text { construct }\end{array}$ & 164.149 & 54 & 3.040 & .000 & .931 & .967 & 0.080 \\
\hline
\end{tabular}

\section{Testing discriminant validity}

After internal reliability, convergent validity and construct validity had been established for the constructs, the next step was to evaluate the discriminant validity. As suggested by Fornell and Larcker (1981), the square roots of AVE were calculated to establish discriminant validity. As presented in Table 5-11, the diagonal values (the square root of AVE) are always greater than the off-diagonal values (correlations of constructs). Therefore, the discriminant validity of the constructs is satisfied.

Once validity and reliability requirements were met, the next sections will examine the hypotheses in turn. 
Table 5-11: Correlation of constructs and the squared root of AVE

\begin{tabular}{|c|c|c|c|c|c|c|c|c|c|c|c|c|c|c|c|}
\hline & Mean & S.D. & (1) & (2) & (3) & (4) & (5) & (6) & (7) & (8) & (9) & (10) & $(11)$ & $(12)$ & (13) \\
\hline 1. $\mathrm{PM}$ & 4.98 & 1.29 & .828 & & & & & & & & & & & & \\
\hline 2. Accountability & 5.45 & 1.12 & $.676^{* *}$ & .766 & & & & & & & & & & & \\
\hline 3. HR Autonomy & 3.34 & 0.91 & $.381^{* *}$ & $.369^{* *}$ & .806 & & & & & & & & & & \\
\hline 4. Entrepreneurial leadership & 5.26 & 1.25 & $.653^{* *}$ & $.663^{* *}$ & $.408^{* *}$ & .801 & & & & & & & & & \\
\hline 5. HR unit competence & 4.64 & 1.52 & $.568^{* *}$ & $.552^{* *}$ & $.339^{* *}$ & $.583^{* *}$ & .901 & & & & & & & & \\
\hline 6. Personal Relation & 4.78 & 1.51 & $-.376^{* *}$ & $-.299^{* *}$ & $-.240^{* *}$ & $-.347^{* *}$ & $-.381^{* *}$ & .840 & & & & & & & \\
\hline 7. Nepotism & 4.12 & 1.64 & $-.355^{* *}$ & $-.292^{* *}$ & $-.274^{* *}$ & $-.382^{* *}$ & $-.324^{* *}$ & $.788^{* *}$ & .875 & & & & & & \\
\hline 8. $\quad$ Training & 4.71 & 1.67 & $.551^{* *}$ & $.515^{* *}$ & $.360^{* *}$ & $.556^{* *}$ & $.750^{* *}$ & $-.370^{* *}$ & $-.357^{* *}$ & 1 & & & & & \\
\hline 9. Communication & 5.13 & 1.41 & $.666^{* *}$ & $.596^{* *}$ & $.391^{* *}$ & $.669^{* *}$ & $.721^{* *}$ & $-.383^{* *}$ & $-.399^{* *}$ & $.634^{* *}$ & .837 & & & & \\
\hline 10. Work motivation & 5.70 & 0.92 & $.435^{* *}$ & $.416^{* *}$ & $.273^{* * *}$ & $.382^{* *}$ & $.369^{* *}$ & $-.115^{*}$ & -.064 & $.377^{* *}$ & $.389^{* *}$ & .817 & & & \\
\hline 11. Org commitment & 5.39 & 1.11 & $.591^{* *}$ & $.473^{* *}$ & $.334^{* *}$ & $.595^{* *}$ & $.615^{* *}$ & $-.322^{* *}$ & $-.284^{* *}$ & $.569^{* *}$ & $.595^{* *}$ & $.583^{* *}$ & .804 & & \\
\hline 12. Job satisfaction & 5.47 & 1.35 & $.548^{* *}$ & $.410^{* *}$ & $.312^{* * *}$ & $.549^{* *}$ & $.591^{* * *}$ & $-.305^{* *}$ & $-.257^{* *}$ & $.515^{* *}$ & $.559^{* *}$ & $.490^{* *}$ & $.827^{* *}$ & 1 & \\
\hline 13. Org - performance & 4.71 & 1.77 & $.441^{* *}$ & $.341^{* *}$ & $.432^{* * *}$ & $.469^{* *}$ & $.514^{* * *}$ & $-.209^{* *}$ & $-.277^{* *}$ & $.399^{* *}$ & $.492^{* *}$ & $.263^{* *}$ & $.468^{* *}$ & $.454^{* * *}$ & .848 \\
\hline
\end{tabular}
Note:

** Correlation is significant at the 0.01 level $(2$ - tailed $)$

* Correlation is significant at the 0.05 level (2 - tailed)

Performance Management (PM) is the average of the five component PM practices with the AVE of 0.685

The bold diagonal values are the square root of AVE for each variable 


\subsubsection{Testing hypotheses}

\subsubsection{Hypothesis 1: the effect of employee PM on outcome variables}

Hypothesis 1 proposes that employee PM is associated with increased perceptions of employee motivation (H1a), organisational commitment (H1b), job satisfaction (H1c) and organisational performance (H1d).

The relationships between the employee PM practice and the outcome effectiveness variables are partially illustrated by the descriptive statistics. Table 5-11 shows that the employee PM practice has positive and significant correlation with employee motivation $(r=0$ $\left..493^{* *}\right)$, organisational commitment $\left(\mathrm{r}=0.593^{* *}\right)$, job satisfaction $\left(\mathrm{r}=0.548^{* *}\right)$, and subsequently organisational performance $\left(r=0.441^{* * *}\right)$. However, in order to test this hypothesis in a more convincing way, the following section is largely dedicated to examining whether the development of employee PM practices can lead to the improvement of employee motivation, organisational commitment, job satisfaction and organisational performance.

\section{Classification according to the development of employee PM}

The cluster analysis technique was initially used to group all respondents based on the five PM practices. In the first step, a hierarchical cluster was performed using Ward's method, which produced a three-cluster solution meeting the condition "the simplest structure possible that still represents homogeneous groupings" (Hair, Black et al. 2006 p.565). In the next step, K-mean cluster analysis was used to carry out the grouping (segmentation) process. As presented in Figure 5-1, the cluster analysis segmented the 322 respondents into three clusters. The first cluster ( $\mathrm{n}=64 ; 18.88$ percent) scores least on all the five PM constructs, i.e. goalbased appraisal $($ mean $=3.52)$; feedback $($ mean $=3.60)$; reward for performance $($ mean $=2.60)$; addressing poor performers $($ mean $=1.98)$; participation $($ mean $=3.20)$. This cluster was labelled "the laggard cluster". The second cluster $(\mathrm{n}=115 ; 35.71$ percent $)$ scores near midpoint across the five constructs, i.e. goal-based appraisal $($ mean $=5.54)$; feedback $($ mean $=5.39)$; reward for performance $($ mean $=4.23)$; addressing poor performers $($ mean $=3.26)$; participation (mean $=5.07)$. This cluster was labelled "the transitional cluster". The third, highest scoring cluster ( $\mathrm{n}=143 ; 44.40$ percent) was labelled "advanced", i.e. goal-based appraisal (mean = $6.30)$; feedback $($ mean $=6.22)$; reward for performance $($ mean $=6.15)$; addressing poor performers $($ mean $=5.77)$; participation $($ mean $=6.02)$. The result from the cluster analysis also indicates that there are significant differences $(\mathrm{P}$ value $<0.001)$ between the three clusters across the five PM practices. 
While all the five PM practices are statistically significant in categorising the clusters, rewarding for performance and addressing poor performers are outstanding in differentiating the advanced cluster from the remainder. In accordance with this result, the respondents in the laggard cluster reported that they disagreed with the statements that their organisation addresses poor performers, as well as seriously rewarding good performers. Similarly, the respondents in the transitional cluster reported that they slightly disagreed with the statement that their organisation seriously addresses poor performers while most had no idea how the organisation rewards good performers.

Figure 5-1: Mean values of five PM practices across the three clusters

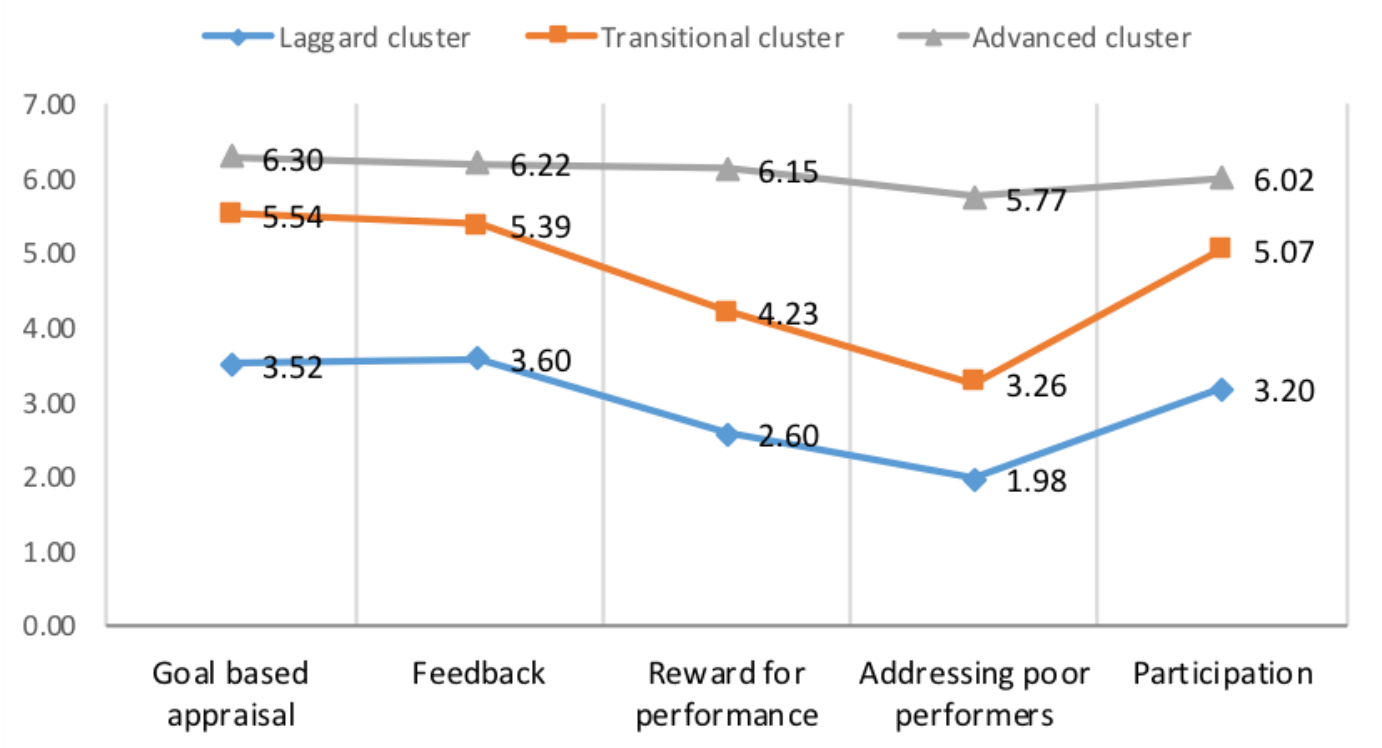

Furthermore, to provide a better understanding of cluster analysis, i.e. examining the consistency among respondents within the same organisation, they were segmented into the three clusters based on the results from the cluster analysis. As can be seen in Table 5-12, although respondents of the same organisation may be distributed in different clusters, there are some notable trends. For example, central administrations such as central ministries tend to engage in laggard PM practices with approximately 40 to 50 percent of respondents falling into the laggard cluster (the group at the end of the list). On the other hand, service delivery institutions with higher self-financing levels have more advanced PM systems. The majority of respondents (50 percent) within these service delivery organisations with totally self-financing mechanism regarded their organisation's PM practices as advanced (the group at the top list). Meanwhile, the respondents from local agencies and partially self-financing institutions tended to see their PM practices as transitional (the middle group in the list). 
The partial heterogeneity between respondents within each organisation can be partially explained by the fact that, although the guidelines for employee PM practices are generally applied to all units in each organisation, implementation depends on the culture and the head of each unit or division. For example, the heads of some units may be open to discussing jobrelated issues with their subordinates while others tend to use autocratic leadership. Our interviews also indicate that distribution of extra salary funds varies from one unit to another, depending on the head of the unit, even though they were within the same organisation. This content will be discussed in detail in the qualitative strand.

Table 5-12: List of organisations with respondents segmented into clusters

\begin{tabular}{|c|c|c|c|c|c|}
\hline Organisation & Category & Financing & Advanced & Transitional & Laggard \\
\hline Organisation 1 & Central-service & Totally self-financed & $90.00 \%$ & $10.00 \%$ & $0.00 \%$ \\
\hline Organisation 2 & Central-service & Totally self-financed & $80.00 \%$ & $10.00 \%$ & $10.00 \%$ \\
\hline Organisation 3 & Central-service & Totally self-financed & $78.57 \%$ & $21.43 \%$ & $0.00 \%$ \\
\hline Organisation 4 & Central-service & Totally self-financed & $66.67 \%$ & $33.33 \%$ & $0.00 \%$ \\
\hline Organisation 5 & Central-service & Totally self-financed & $60.00 \%$ & $20.00 \%$ & $20.00 \%$ \\
\hline Organisation 6 & Central-service & Totally self-financed & $57.14 \%$ & $14.29 \%$ & $28.57 \%$ \\
\hline Organisation 7 & Central-service & Totally self-financed & $57.14 \%$ & $28.57 \%$ & $14.29 \%$ \\
\hline Organisation 8 & Provincial-admin & State financed & $56.25 \%$ & $37.50 \%$ & $6.25 \%$ \\
\hline Organisation 9 & Provincial-service & Totally self-financed & $54.55 \%$ & $45.45 \%$ & $0.00 \%$ \\
\hline Organisation 10 & Provincial-admin & State financed & $50.00 \%$ & $40.00 \%$ & $10.00 \%$ \\
\hline Organisation 11 & Central-service & Partially self-financed & $50.00 \%$ & $30.00 \%$ & $20.00 \%$ \\
\hline Organisation 12 & Provincial-service & Partially self-financed & $45.45 \%$ & $27.27 \%$ & $27.27 \%$ \\
\hline Organisation 13 & Central-service & Partially self-financed & $45.45 \%$ & $45.45 \%$ & $9.09 \%$ \\
\hline Organisation 14 & Central-service & Partially self-financed & $40.00 \%$ & $40.00 \%$ & $20.00 \%$ \\
\hline Organisation 15 & Central-service & Partially self-financed & $37.50 \%$ & $37.50 \%$ & $25.00 \%$ \\
\hline Organisation 16 & Central-service & Partially self-financed & $33.33 \%$ & $33.33 \%$ & $33.33 \%$ \\
\hline Organisation 17 & Central-service & Partially self-financed & $30.77 \%$ & $53.85 \%$ & $15.38 \%$ \\
\hline Organisation 18 & Provincial-admin & Partially self-financed & $0.00 \%$ & $100.00 \%$ & $0.00 \%$ \\
\hline Organisation 19 & Central-service & Partially self-financed & $25.00 \%$ & $55.00 \%$ & $20.00 \%$ \\
\hline Organisation 20 & Central-admin & State financed & $22.22 \%$ & $55.56 \%$ & $22.22 \%$ \\
\hline Organisation 21 & Central-admin & State financed & $14.29 \%$ & $85.71 \%$ & $0.00 \%$ \\
\hline Organisation 22 & Central-service & Partially self-financed & $12.50 \%$ & $43.75 \%$ & $43.75 \%$ \\
\hline Organisation 23 & Central-service & Partially self-financed & $11.11 \%$ & $55.56 \%$ & $33.33 \%$ \\
\hline Organisation 24 & Central-admin & State financed & $29.17 \%$ & $45.83 \%$ & $25.00 \%$ \\
\hline Organisation 25 & Central-admin & State financed & $28.57 \%$ & $42.86 \%$ & $28.57 \%$ \\
\hline
\end{tabular}




\begin{tabular}{|l|l|l|l|l|l|}
\hline Organisation 26 & Provincial-admin & State financed & $29.41 \%$ & $29.41 \%$ & $41.18 \%$ \\
\hline Organisation 27 & Central-admin & State financed & $14.29 \%$ & $42.86 \%$ & $42.86 \%$ \\
\hline Organisation 28 & Central-admin & State financed & $17.65 \%$ & $41.18 \%$ & $41.18 \%$ \\
\hline Organisation 29 & Central-admin & State financed & $25.00 \%$ & $25.00 \%$ & $50.00 \%$ \\
\hline
\end{tabular}

Notes:

Central-service: Public service delivery organisation at the central level

Provincial-service: Public service delivery organisation at the provincial level

Central-admin: Administration organisation at the central level

Provincial-admin: Administration organisation at the provincial level

Totally self-financed: The organisation can cover its all expenditures (no state budget needed)

Partially self-financed: The organisation can cover partially its all expenditures

State-financed: The organisation's all expenditure is covered by the state budget.

\section{Testing the difference in the outcome variables across the clusters}

In the next step of testing Hypothesis 1, this research used one-way ANOVA to examine whether there are significant differences existing among the outcome variables across the clusters. Table 5-13 shows that there are significant differences in work motivation, organisational commitment, job satisfaction and organisational performance across the three clusters. Employees in the laggard cluster have lower perceived motivation and commitment, and are less satisfied with their jobs than employees in the transitional and advanced clusters.

Table 5-13: Mean value of outcomes across three clusters

\begin{tabular}{cccccc}
\hline & $\begin{array}{c}\text { Laggard } \\
\text { cluster }\end{array}$ & $\begin{array}{c}\text { Transitional } \\
\text { cluster }\end{array}$ & $\begin{array}{c}\text { Advanced } \\
\text { cluster }\end{array}$ & F & Significant \\
\hline Work motivation & 5.026 & 5.664 & 6.037 & 25.233 & .000 \\
Org commitment & 4.359 & 5.319 & 5.919 & 53.588 & .000 \\
Job satisfaction & 4.296 & 5.469 & 6.986 & 44.127 & .000 \\
Org performance & 3.665 & 4.514 & 5.337 & 23.891 & .000 \\
Valid N & 64 & 115 & 143 & & \\
\hline
\end{tabular}

Similarly, the table shows that the scores for four outcome variables are highest in the advanced cluster that has had radical innovation of the five PM practices. For the transitional cluster, the values for the four outcome variables are higher than the respective values in the laggard cluster but lower than the value in the advanced cluster. The differences in each outcome variable across the three clusters are all statistically significant, with $\mathrm{p}<0.001$. These results imply that there are significant differences in employee motivation (H1a), organisational commitment (H1b), job satisfaction (H1c) and organisational performance (H1d) across the three clusters. In other words, the more that organisations adopt advanced PM practices, the 
better employee motivation, organisational commitment, job satisfaction and organisational performance they enjoy. Hence, Hypothesis 1 is supported.

\subsubsection{Hypothesis 2: the effect of contextual variables on employee PM}

Hypothesis 2 hypothesises that agency accountability (H2a), HR autonomy (H2b), entrepreneurial leadership $(\mathrm{H} 2 \mathrm{c})$ and $\mathrm{HR}$ competence $(\mathrm{H} 2 \mathrm{~d})$ are associated with the development of employee PM practices.

As stated in the preceding chapter, this hypothesis was tested by multinomial logistic regression. Before running this regression, it is advisable to examine multicollinearity because this problem can affect the accuracy of parameters in a regression model (Field 2009). Given that multinomial logistic regression does not allow the checking of multicollinearity, a linear regression analysis was conducted instead. In this analysis, employee PM is the dependent variable and the contextual variables are the independent variables. The result from diagnosing multicollinearity indicates that the Variance Inflation Factor (VIF) values of agency accountability, HR autonomy, entrepreneurial leadership and HR competence are 1.951, 1.239, 2.101 and 1.654 respectively. This indicates that there are not serious collinearity problems because all these values are below the threshold of 2.5 (Field 2009).

Table 5-14 reports the results of two multinomial logistic regressions with the transitional cluster as the reference category. The first part of each model compares the transitional cluster with the laggard cluster. The second part of the models compares the advanced cluster with the transitional cluster. The first model contains only the four main variables while the second model incorporates the interaction terms of HR competence with the three remaining variables.

As shown in model 1, the set of antecedents significantly predicts differences across the clusters with $\chi^{2}(8, \mathrm{~N}=322)=173.936, \mathrm{p}<0.001$. The estimated pseudo R-square shows that these contextual variables account for 47.6 percent of the variation of the clusters. Additionally, by looking at the coefficients (B), we can see that accountability (H1a), entrepreneurial leadership (H1b) and HR autonomy (H1c), but not HR competence (H1d), are statistically significantly associated with the evolution of PM systems.

Although the effect of HR competence is not significant in model 1, based on the initial qualitative data, this research infers that this variable can have a significant influence if it interacts with other variables i.e. accountability, HR autonomy and entrepreneurial leadership. Therefore, three interaction terms between HR competency and the three remaining variables were included in model 2. The result indicates that entrepreneurial leadership reinforces 
(moderating) the relationship between HR competence and the development of employee PM. However, accountability and HR autonomy are not significant in reinforcing this relationship.

Table 5-14: Multinomial regression of contextual variables

\begin{tabular}{|c|c|c|c|c|c|c|c|c|}
\hline \multirow[t]{3}{*}{ Predictor } & \multicolumn{4}{|c|}{ Model 1} & \multicolumn{4}{|c|}{ Model 2} \\
\hline & \multicolumn{2}{|c|}{$\begin{array}{c}\text { Transitional vs. } \\
\text { Laggard }\end{array}$} & \multicolumn{2}{|c|}{$\begin{array}{c}\text { Advanced vs. } \\
\text { Transitional }\end{array}$} & \multicolumn{2}{|c|}{$\begin{array}{l}\text { Transitional vs. } \\
\text { Laggard }\end{array}$} & \multicolumn{2}{|c|}{$\begin{array}{l}\text { Advanced vs. } \\
\text { Transitional }\end{array}$} \\
\hline & B & OR & $\mathrm{B}$ & OR & B & OR & B & OR \\
\hline \multirow[t]{2}{*}{ Intercept } & $-4.625 * * *$ & & $-8.349 * * *$ & & $5.899 * * *$ & & $-8.525 * * *$ & \\
\hline & $(1.030)$ & & $(1.267)$ & & $(1.624)$ & & $(1.263)$ & \\
\hline \multirow{2}{*}{$\begin{array}{l}\text { Accountability } \\
(\mathrm{H} 2 \mathrm{a})\end{array}$} & $\mathbf{0 . 5 8 5}^{* *}$ & 1.795 & $.808 * * *$ & 2.243 & $.633 *$ & .531 & $.740 * *$ & 2.096 \\
\hline & $(0.204)$ & & $(.211)$ & & $(.268)$ & & $(.217)$ & \\
\hline \multirow{2}{*}{$\begin{array}{l}\text { HR autonomy } \\
\text { (H2b) }\end{array}$} & -0.235 & .791 & $.508 * *$ & 1.663 & -.227 & 1.255 & $.495 * *$ & 1.640 \\
\hline & $(0.205)$ & & $(.186)$ & & $(.208)$ & & $(.192)$ & \\
\hline \multirow{2}{*}{$\begin{array}{l}\text { Entre leadership } \\
(\mathrm{H} 2 \mathrm{c})\end{array}$} & $0.537 * *$ & 1.710 & .261 & 1.298 & $.637 * *$ & .529 & .382 & 1.466 \\
\hline & $(0.184)$ & & $(.180)$ & & $(.230)$ & & $(.200)$ & \\
\hline \multirow{2}{*}{$\begin{array}{l}\text { HR competence } \\
(\mathrm{H} 2 \mathrm{~d})\end{array}$} & .155 & .857 & .152 & 1.167 & .274 & .760 & .096 & 1.101 \\
\hline & $(0.138)$ & & $(.123)$ & & $(.164)$ & & $(.131)$ & \\
\hline \multirow{2}{*}{$\begin{array}{l}\text { HR competence } \\
\text { x Accountability }\end{array}$} & & & & & .078 & .925 & .193 & 1.213 \\
\hline & & & & & $(.252)$ & & $(.216)$ & \\
\hline \multirow{2}{*}{$\begin{array}{l}\text { HR competence } \\
\text { x HR autonomy }\end{array}$} & & & & & .126 & .881 & .048 & 1.049 \\
\hline & & & & & $(.145)$ & & $(.134)$ & \\
\hline \multirow{2}{*}{$\begin{array}{l}\text { HR competence } \\
\text { x Entre leadership }\end{array}$} & & & & & .083 & .920 & $.476 *$ & 1.609 \\
\hline & & & & & $(.228)$ & & $(.201)$ & \\
\hline Chi-square & \multicolumn{4}{|c|}{$173.936 * * *$} & \multicolumn{4}{|c|}{$186.364 * * *$} \\
\hline $\mathrm{DF}$ & \multicolumn{4}{|c|}{8} & \multicolumn{4}{|c|}{14} \\
\hline Pseudo R-square & \multicolumn{4}{|c|}{0.476} & \multicolumn{4}{|c|}{.501} \\
\hline Sample size & \multicolumn{4}{|c|}{322} & \multicolumn{4}{|c|}{322} \\
\hline
\end{tabular}

Notes:

$-*$ if $\mathrm{P}_{\text {value }}=<0.05 ; * *$ if $\mathrm{P}_{\text {value }}=<0.01 ; * * *$ if $\mathrm{P}_{\text {value }}=<0.001$

- Values in brackets are standard errors

- The reference category is the transitional cluster.

While the beta coefficients (B) indicate the direction and significance of one contextual variable on the likelihood of introducing one cluster in relation to another, odds-ratios (OR) imply the probability of adopting one cluster compared to another. Accordingly, for each unit of increased accountability, the likelihood of moving into the advanced cluster is 2.243 times greater than into the transitional cluster. With a similar increase, the likelihood of moving into the transitional cluster is 1.795 times greater than into the laggard cluster. In other words, one unit of increased accountability increases the likelihood of adopting advanced PM practices 
rather than transitional PM practices by 124.3 percent (2.243-1). Meanwhile, the likelihood of adopting transitional PM practices compared to laggard PM practices increases by 70.9 percent $(1.795-1)$.

With regard to the effect of HR autonomy, each unit of HR autonomy increases, the likelihood of experiencing an advanced PM system against a transitional PM system increases 1.663 times. In other words, a one-unit increase in HR autonomy increases the likelihood of introducing an advanced PM system compared to a transitional PM system by 66.3 percent.

Likewise, when entrepreneurial leadership increases one unit, the likelihood of engaging the transitional cluster compared to the laggard cluster is 1.710 times greater. In other words, a one-unit increase in entrepreneurial leadership increases the likelihood of adopting transitional PM practices rather than laggard PM practices by 71 percent.

As stated above, although HR competence itself is not significant, the product term between HR competence and entrepreneurial leadership is significant in predicting the development of employee PM. Accordingly, each unit of the product term increases, the likelihood of experiencing an advanced PM system against a transitional PM system increases 1.609 times.

In summary, the analysis results indicate that accountability, entrepreneurial leadership and HR autonomy significantly predict the development of employee PM. Although HR competence by itself does not directly relate to the development of employee PM, the interaction between HR competence and entrepreneurial leadership can significantly predict this development.

\subsubsection{Hypothesis 3: the effect of contextual variables on the outcome variables}

Hypothesis 3 states that agency accountability (H3a), HR autonomy (H3b), entrepreneurial leadership (H3c) and HR competence (H3d) indirectly affect employee motivation, organisational commitment, job satisfaction and organisational performance through the mediation role of employee PM.

Testing Hypothesis 2 reveals that the development of employee PM is only driven by agency accountability, HR autonomy, and entrepreneurial leadership. Therefore, this section only focuses on examining the indirect effect of these three factors.

As discussed in the previous chapter, the structural equation modelling (SEM) technique of Amos was adopted to test this hypothesis. Accordingly, observed variable path analysis was 
used to examine direct and indirect effects, and the fit of the hypothesised model. As can be observed in Figure 5-2, direct effects are path coefficients between an exogenous variable and an endogenous variable. For instance, the direct effect of accountability on employee PM is 0.47 while the direct effect of employee PM on motivation is 0.36 . The indirect effect of accountability on motivation can be computed by multiplying these two coefficients. Accordingly, the indirect effect of accountability on motivation is $0.170(0.47 * 0.36)$.

\section{Figure 5-2: Initial path analysis}

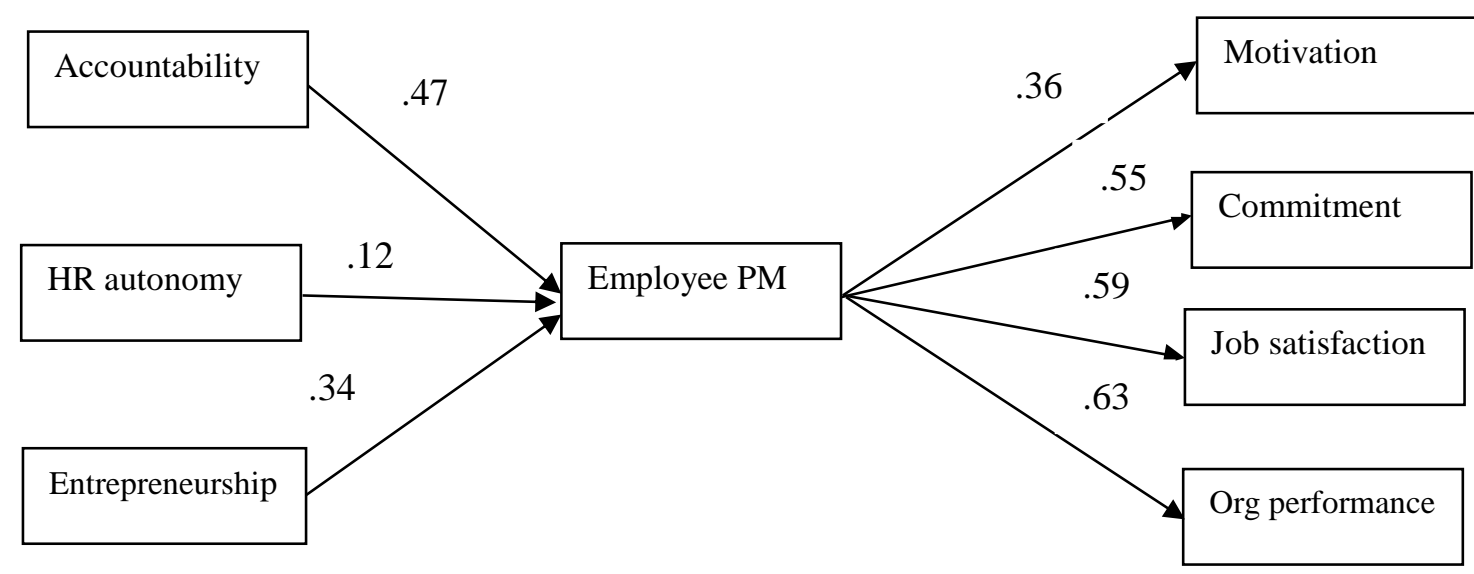

Having obtained the indirect effect coefficients, it is very important to know whether these indirect effects are significant. Therefore, bootstrap with the bias-corrected percentile method was performed. The result of examining statistical significance is presented in Table 515. This indicates that all three contextual factors significantly affect the outcome variables indirectly through the mediator of employee PM.

Table 5-15: Indirect effects of contextual factors

\begin{tabular}{|l|c|c|c|}
\hline & Accountability & HR autonomy & $\begin{array}{c}\text { Entrepreneurial } \\
\text { leadership }\end{array}$ \\
\hline Work motivation & $.170^{* * *}$ & $.043^{*}$ & $.122^{* * * *}$ \\
\hline Organisational commitment & $.260^{* * *}$ & $.066^{*}$ & $.186^{* * *}$ \\
\hline Job satisfaction & $.279^{* * *}$ & $.071^{*}$ & $.200^{* * *}$ \\
\hline Organisational performance & $.296^{* * *}$ & $.075^{*}$ & $.212^{* * *}$ \\
\hline
\end{tabular}

Note:

* Indirect effect is significant at the 0.05 level (2-tailed)

** Indirect effect is significant at the 0.01 level (2-tailed)

$* * *$ Indirect effect is significant at the 0.001 level (2-tailed) 
Although the indirect effects are statistically significant as hypothesised, the indices of the model fit were not very good, i.e. $\chi 2 / \mathrm{df}=7.508, \mathrm{GFI}=0.939, \mathrm{CFI}=0.941, \mathrm{RMSEA}=$ 0.142. Modification indices indicated that the contextual variables have not only indirect effects via employee PM but also direct effects on the outcome variables. Specifically, the modification indices suggested that, apart from indirect effects, accountability has a direct effect on work motivation, and HR autonomy has a direct effect on organisational performance. Meanwhile, entrepreneurial leadership has direct effects on organisational commitment, job satisfaction and organisational performance. After these direct relationships were established, the modified model was recalculated with path coefficients as Figure 5-3 below.

\section{Figure 5-3: Modified path analysis}

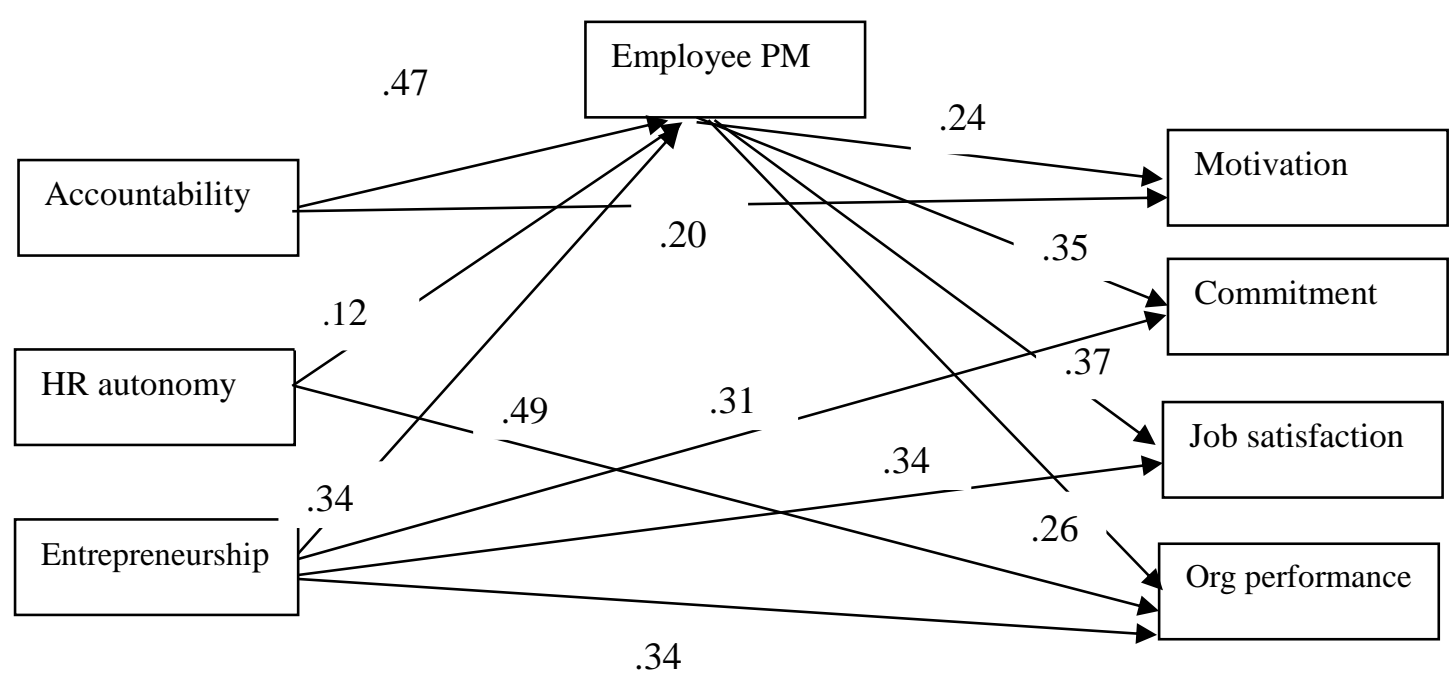

The modified model provides a very good fit for the data $(\chi 2 / \mathrm{df}=1.200$, GFI $=0.994$, $\mathrm{CFI}=0.999, \mathrm{RMSEA}=0.025)$. Table 5-16 below shows direct and indirect effects of the contextual factors on employee PM and the outcome variables. Accordingly, each contextual variable has indirect effects on all four outcome variables. However, the direct effects vary among the contextual factors. Specifically, entrepreneurial leadership has three direct effects (excluding employee PM) while accountability and HR autonomy only have one direct effect. 
Table 5-16: Direct and indirect effects of the modified model

\begin{tabular}{|l|l|l|l|l|l|l|}
\hline & \multicolumn{2}{|c|}{ Accountability } & \multicolumn{2}{c|}{ HR autonomy } & \multicolumn{2}{c|}{$\begin{array}{c}\text { Entrepreneurial } \\
\text { leadership }\end{array}$} \\
\hline & Direct & Indirect & Direct & Indirect & Direct & Indirect \\
\hline Employee PM & $.47^{* *}$ & NA & $.12^{*}$ & NA & $.34^{* * *}$ & NA \\
\hline Work motivation & $.20^{* *}$ & $.11^{* * *}$ & .000 & $.03^{*}$ & .000 & $.08^{* * *}$ \\
\hline Commitment & .000 & $.16^{* * *}$ & .000 & $.04^{*}$ & $.31^{* * *}$ & $.12^{* * *}$ \\
\hline Job satisfaction & .000 & $.18^{* * *}$ & .000 & $.05^{*}$ & $.34^{* * *}$ & $.13^{* * *}$ \\
\hline $\begin{array}{l}\text { Organisational } \\
\text { performance }\end{array}$ & .000 & $.12^{* *}$ & $.49^{* * *}$ & $.03^{*}$ & $.34^{* *}$ & $.09^{* *}$ \\
\hline
\end{tabular}

Note:

*** Direct effect is significant at the 0.001 level $(2$ - tailed $)$

** Direct effect is significant at the 0.01 level (2 - tailed)

* Direct effect is significant at the 0.05 level (2 - tailed)

In short, the results from the SEM analysis provide some noteworthy findings. First, they indicate that accountability, HR autonomy and entrepreneurial leadership have indirect effects on the four outcome variables: employee motivation, organisational commitment, job satisfaction and organisational performance. Accordingly, employee PM plays a critical role in transferring the effect of the contextual variables on the outcome variables. Second, the results reveal that the contextual variables not only have indirect effects as hypothesised but also have direct effects on the outcome variables. Third, the results also provide more evidence for the preceding conclusions that accountability, HR autonomy and entrepreneurial leadership significantly drive the development of employee PM while employee PM in turn has significant effects on the outcome variables. Finally, as a notable virtue of SEM, the indices of the model fit allow us to conclude that the proposed theoretical model has a good fit with the collected data.

\subsubsection{Hypothesis 4: the moderation role of cultural variables}

Hypothesis 4 posits that the relationships between the contextual factors and employee PM are moderated by the cultural variables of nepotism $(\mathrm{H} 4 \mathrm{a})$, and interpersonal relationships (H4b).

Accordingly, the research aims to examine if nepotism and interpersonal relationships can play a moderator role in the relationships between the contextual variables and employee PM. According to Baron and Kenny (1986), a moderator is understood as a variable that affects the direction and/or strength of the relationship between an independent and a dependent variable. The independent variables in this hypothesis are agency accountability, HR autonomy 
and entrepreneurial leadership. Meanwhile, the dependent variable is employee PM. Consistent with previous research, e.g. (Kinicki, Jacobson et al. 2013) and (Cho and Lee 2011), this analysis treats employee PM as a single construct comprising the five practices as a coherent set of practices (Cronbach's alpha: 0.878).

As presented in the fourth chapter, the procedure for testing moderation effects includes three regression equations as suggested by Zedeck (1971) with the aid of SPSS version 23. To minimise the problem of multicollinearity, this research firstly standardised the independent and moderation variables before creating the interaction terms. In the first regression equation (step 1), only one independent variable (contextual variable) was entered after controlling demographic variables, namely age, gender, education and managerial position. Then a moderation variable (cultural variable) was added in the second regression equation (step 2). Finally, the interaction between the independent variable and the independent variable was added in the third regression equation (step 3). The hypothesis of a moderation effect is supported if the $\mathrm{F}$ values related to the change in $\mathrm{R}^{2}$ in step 2 and step 3 are significantly different from zero (Zedeck 1971; Cohen and Cohen 1975). Additionally, the hypothesis is also supported if the beta coefficients of the independent and moderating variable in the first and second regression equation and the beta coefficient of the interaction term in the third regression equation significantly vary from zero (Baron and Kenny 1986).

The results, as presented in Table 5-17 and Table 5-18, reveal that there are strong and positive relationships between the contextual variables and employee PM. Specifically, each contextual variable explains a significant proportion of variance in employee PM, i.e. accountability $\left(\Delta \mathrm{R}^{2}=0.429\right)$, HR autonomy $\left(\Delta \mathrm{R}^{2}=0.145\right)$, entrepreneurial leadership $\left(\Delta \mathrm{R}^{2}=\right.$ 0.388). Additionally, $\Delta \mathrm{R}^{2}$ gained by including the moderating variables (interpersonal relationships and nepotism) is always greater than zero at the $\mathrm{P}$ value $<0.001$.

However, only two out of six interaction terms are significant at the $\mathrm{P}$ value $<0.05$ and one interaction term has weak significance at the $\mathrm{P}$ value of 0.076 . These are:

1) the interaction between nepotism and autonomy $\left(B=-.147^{* *}, \Delta R^{2}=0.013\right.$ and $P$ value $=0.02)$.

2) the interaction between personal relationships and entrepreneurial leadership $(B=-$ $.125^{* *}, \Delta \mathrm{R}^{2}=0.008$ with $\mathrm{P}$ value $\left.=0.031\right)$.

3) the interaction between nepotism and entrepreneurial leadership ( $B=-.098^{*}, \Delta R^{2}=$ 0.005 with $\mathrm{P}$ value $=0.076$ ). 
Accordingly, the relationship between HR autonomy and employee PM is moderated by nepotism while the relationship between entrepreneurial leadership and employee PM is moderated by personal relationships and nepotism. In other words, the relationship between HR autonomy and employee PM is significantly weaker for organisations with high levels of nepotism. Meanwhile, the relationship between entrepreneurial leadership and the development of employee PM is significantly weakened when personal relationships and nepotism are highly rated. The analysis results also reveal that no cultural factor has a moderating effect on the accountability-employee PM relationship.

Table 5-17: Results of moderated regression analysis for nepotism (The dependent variable is employee PM)

\begin{tabular}{llccccc}
\hline Step & \multicolumn{1}{c}{ Variables } & $\mathbf{B}$ & $\mathbf{R}^{\mathbf{2}}$ & $\Delta \mathbf{R}^{\mathbf{2}}$ & $\begin{array}{c}\text { F- } \\
\text { change }\end{array}$ & $\begin{array}{c}\text { Sign. } \mathbf{F} \\
\text { change }\end{array}$ \\
\hline 1 & Accountability & $.761^{* * *}$ & .484 & .429 & 262.225 & .000 \\
2 & Nepotism & $-.129^{* * *}$ & .507 & .024 & 15.162 & .000 \\
3 & Accountability*Nepotism & -.067 & .510 & .003 & 1.867 & .173 \\
\hline 1 & HR autonomy & $.543^{* * *}$ & .200 & .145 & 57.260 & .000 \\
2 & Nepotism & $-.201^{* * *}$ & .258 & .058 & 24.847 & .000 \\
3 & HR autonomy *Nepotism & $\mathbf{- . 1 4 7 ^ { * * }}$ &. $\mathbf{2 7 1}$ & $\mathbf{. 0 1 3}$ & $\mathbf{5 . 4 9 9}$ & $\mathbf{. 0 2 0}$ \\
\hline 1 & Entrepreneurship & $.655^{* * *}$ & .443 & .388 & 219.664 & .000 \\
2 & Nepotism & $-.095^{* * *}$ & .455 & .012 & 7.047 & .008 \\
3 & Entrepreneurship $*$ Nepotism & $\mathbf{- . 0 9 8}^{*}$ & $\mathbf{. 4 6 0}$ & $\mathbf{. 0 0 5}$ & $\mathbf{3 . 1 7 7}$ & $\mathbf{. 0 7 6}$ \\
\hline
\end{tabular}

Note: $* \mathrm{P}<0.1 ; * * \mathrm{P}<0.05 ; * * * \mathrm{P}<0.01$

Table 5-18: Results of moderated regression analysis for personal relationships (The dependent variable is employee PM)

\begin{tabular}{|c|c|c|c|c|c|c|}
\hline Step & Variables & $\overline{\mathbf{B}}$ & $\mathbf{R}^{2}$ & $\Delta \mathbf{R}^{2}$ & $\begin{array}{c}\text { F- } \\
\text { change }\end{array}$ & $\begin{array}{l}\text { Sign. F } \\
\text { change }\end{array}$ \\
\hline $\mathbf{1}$ & Accountability & $.761^{* * * *}$ & .484 & .429 & 262.225 & .000 \\
\hline 2 & Relationship & $-.153^{* * *}$ & .512 & .028 & 18.266 & .000 \\
\hline 3 & Accountability*Relationship & -.069 & .515 & .003 & 1.960 & .162 \\
\hline 1 & Autonomy & $.543^{* * * *}$ & .200 & .145 & 57.260 & .000 \\
\hline 2 & Relationship & $-.238^{* * *}$ & .271 & .071 & 30.610 & .000 \\
\hline 3 & Autonomy *Relationship & -.053 & .273 & .002 & .739 & .391 \\
\hline
\end{tabular}




\begin{tabular}{llccccc}
\hline $\mathbf{1}$ & Entrepreneurship & $.655^{* * *}$ & .443 & .388 & 219.664 & .000 \\
2 & Relationship & $-.139^{* * *}$ & .465 & .022 & 13.516 & .000 \\
3 & Entrepreneurship *Relationship & $\mathbf{- . 1 2 5}^{* *}$ & $\mathbf{. 4 7 3}$ & $\mathbf{. 0 0 8}$ & $\mathbf{4 . 6 8 6}$ & $\mathbf{. 0 3 1}$ \\
\hline
\end{tabular}

Note: $* \mathrm{P}<0.1 ; * * \mathrm{P}<0.05 ; * * * \mathrm{P}<0.01$

To describe the moderation effect of nepotism on the HR autonomy-employee PM relationship in a graph, the levels of employee PM were plotted against high and low levels of nepotism. As presented in Figure 5-4, when nepotism levels are high, the relationship between HR autonomy and employee PM is weaker. Specifically, with the same score for HR autonomy, the scores for employee PM are lower when nepotism levels are high. In other words, the positive effect of HR autonomy is weakened by a perception of high nepotism.

Figure 5-4: Effect of nepotism on the HR autonomy-employee PM relationship

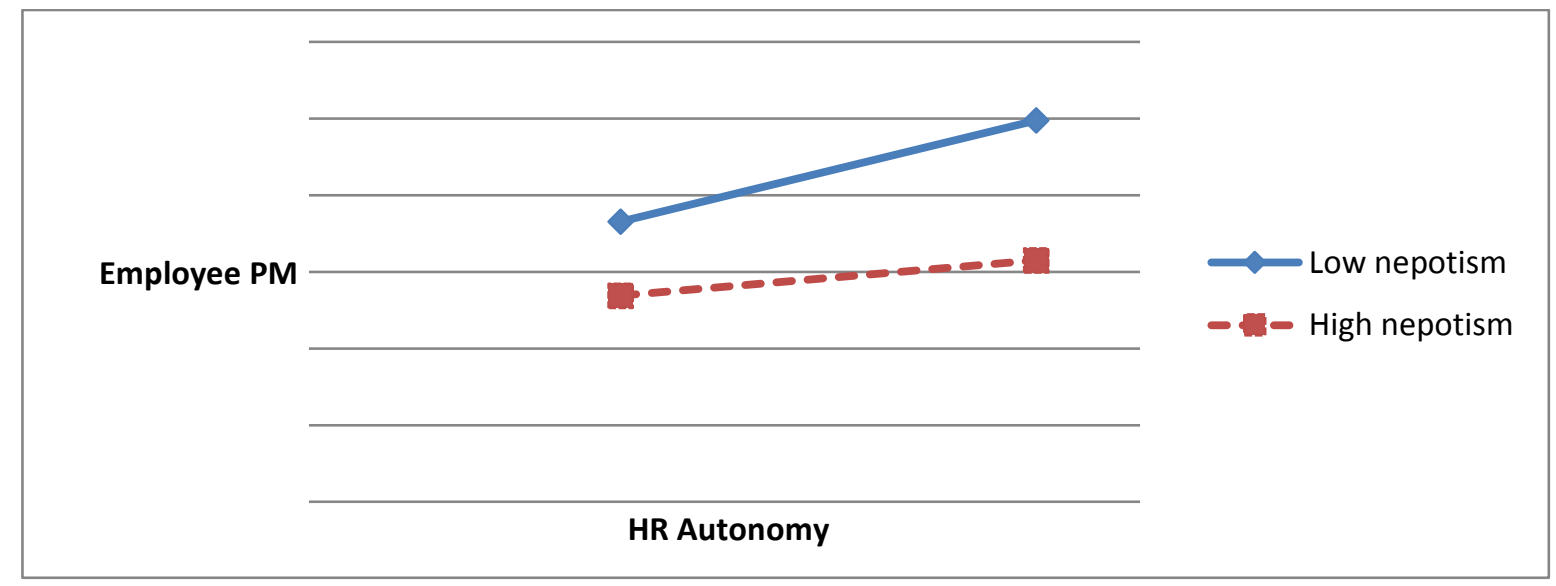

Similarly, the moderation effect of personal relationships and nepotism on the entrepreneurship-employee PM relationship is expressed in Figure 5-5 and Figure 5-6. When personal relationships and nepotism levels are high, the relationship between entrepreneurial leadership and employee PM is weakened. The graphs show that at the same score for entrepreneurial leadership, the score for employee PM tends to decrease when the perception of personal relationships and nepotism levels is high. 
Figure 5-5: Effect of personal relationships on the entrepreneurship-employee PM relationship

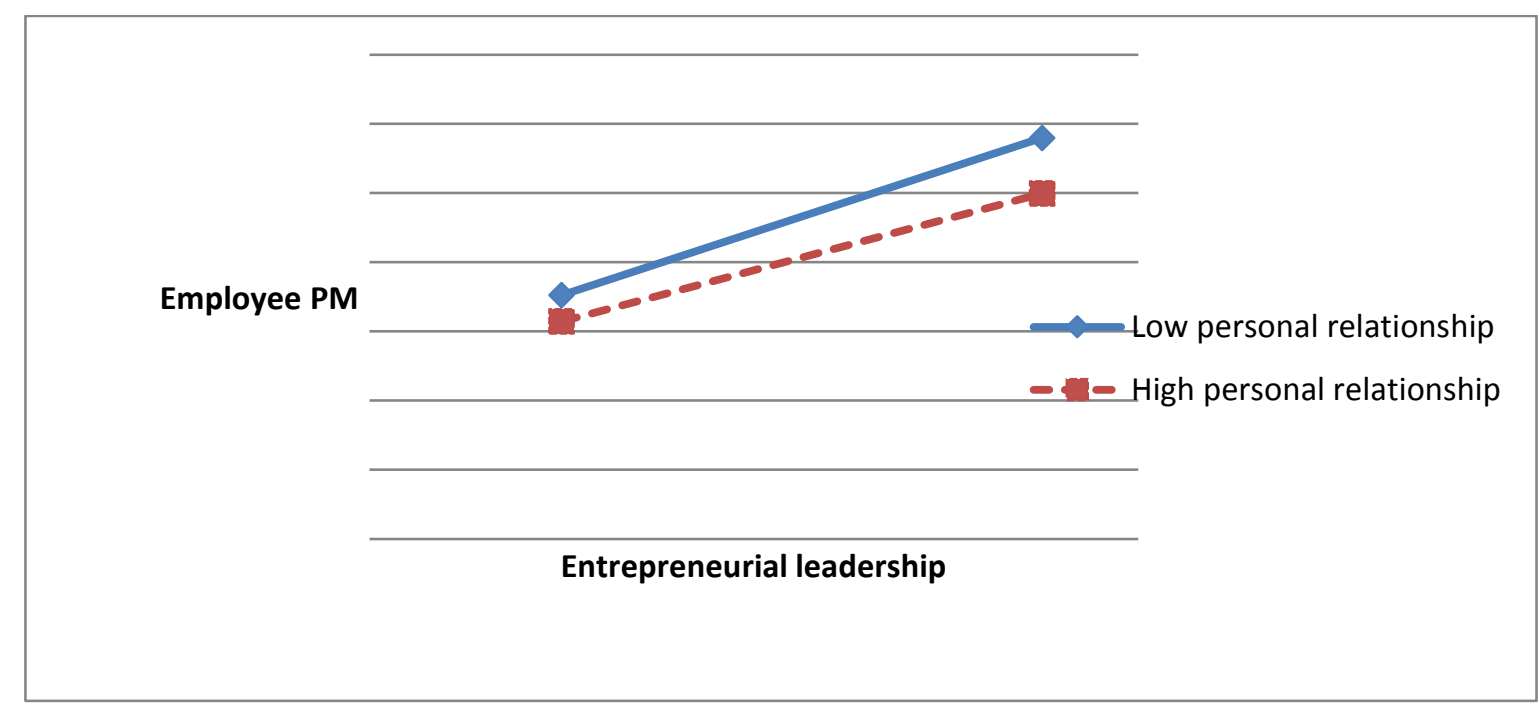

Figure 5-6: Effect of nepotism on the entrepreneurship-employee PM relationship

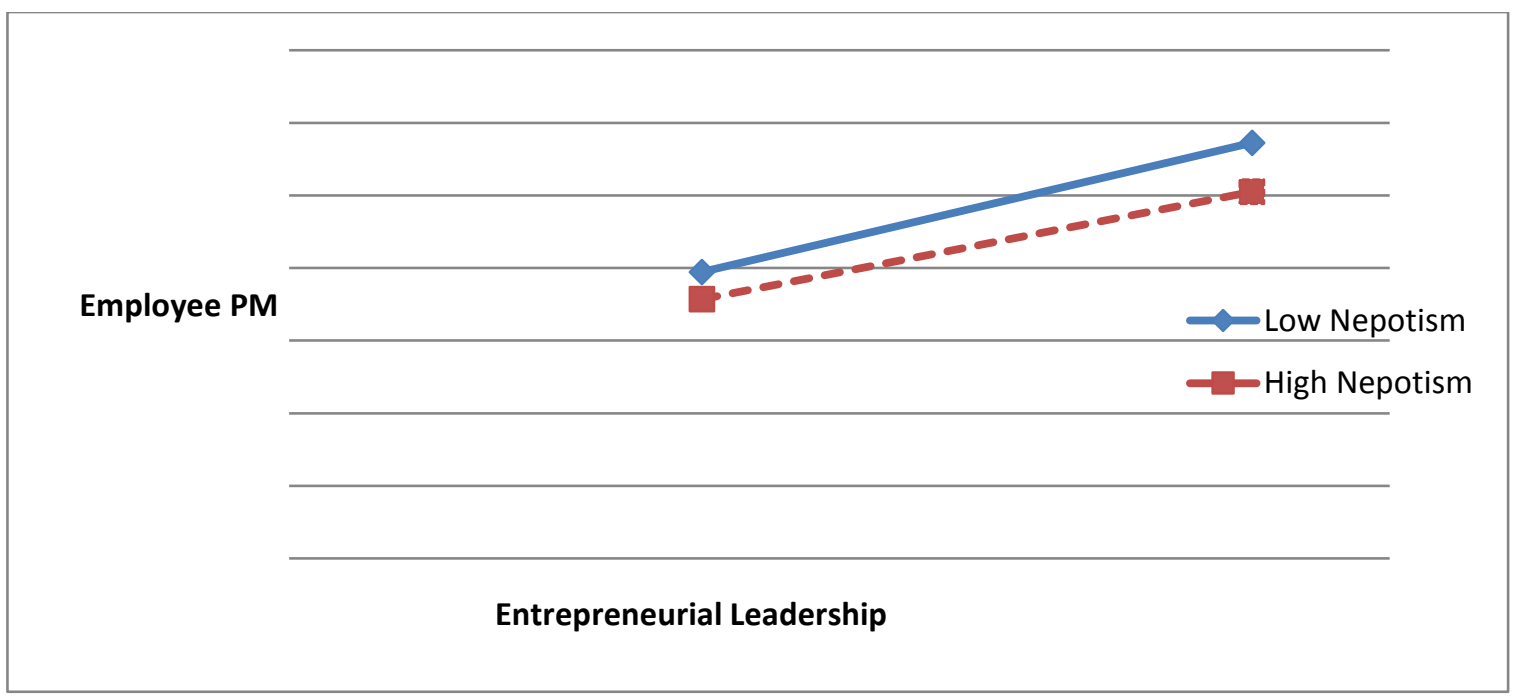

In sum, Hypothesis 4 is partially supported by the analysis. More specifically, nepotism and interpersonal relationships significantly moderate the HR autonomy - employee PM relationship and the entrepreneurial leadership - employee PM relationship. However, they fail to moderate the accountability-employee PM relationship.

\subsubsection{Hypothesis 5: the moderation role of intervention variables}

Hypothesis 5 posits that the relationships between the contextual factors and employee PM are moderated by communication (H5a), and training (H5b). 
As described in Table 5-19, communication significantly moderates the HR autonomyemployee PM relationship $\left(\Delta \mathrm{R}^{2}=0.008\right.$ with $\mathrm{P}$ value $\left.=0.031\right)$; and the entrepreneurshipemployee PM relationship $\left(\Delta \mathrm{R}^{2}=0.012\right.$ with $\mathrm{P}$ value $\left.=0.007\right)$. Meanwhile, as shown in Table 5-20, training significantly moderates the entrepreneurship-employee PM relationship $\left(\Delta \mathrm{R}^{2}=\right.$ 0.007 with $\mathrm{P}$ value $=0.039$ ). Interestingly, like the cultural variables, no intervention practice has a moderating effect on the accountability - employee PM relationship.

Table 5-19: Results of moderated regression analysis for communication (The dependent variable is employee PM)

\begin{tabular}{|c|c|c|c|c|c|c|}
\hline Step & Variables & $\mathbf{B}$ & $\mathbf{R}^{2}$ & $\Delta \mathbf{R}^{2}$ & $\begin{array}{c}\text { F- } \\
\text { change }\end{array}$ & $\begin{array}{l}\text { Sign. F } \\
\text { change }\end{array}$ \\
\hline 1 & Accountability & $0.761^{* * * *}$ & .484 & .429 & 262.225 & .000 \\
\hline 2 & Communication & $.327^{* * * *}$ & .567 & .083 & 60.606 & .000 \\
\hline 3 & Accountability* Communication & .014 & .567 & .000 & .112 & .738 \\
\hline 1 & Autonomy & $.543^{* * *}$ & .200 & .145 & 57.260 & .000 \\
\hline 2 & Relationship & $.507^{* * *}$ & .446 & .246 & 139.845 & .000 \\
\hline 3 & Autonomy $*$ Communication & $.106^{* *}$ & .454 & .008 & 4.721 & .031 \\
\hline $\mathbf{1}$ & Entrepreneurship & $.655^{* * *}$ & .443 & .388 & 219.664 & .000 \\
\hline 2 & Relationship & $.332^{* * * *}$ & .518 & .075 & 49.017 & .000 \\
\hline 3 & Entrepreneurship $*$ Communication & $.129^{* * * *}$ & .529 & .012 & 7.930 & .007 \\
\hline
\end{tabular}

Table 5-20: Results of moderated regression analysis for training (The dependent variable is employee PM)

\begin{tabular}{llccccc}
\hline Step & \multicolumn{1}{c}{ Variables } & $\mathbf{B}$ & $\mathbf{R}^{\mathbf{2}}$ & $\Delta \mathbf{R}^{\mathbf{2}}$ & $\begin{array}{c}\text { F- } \\
\text { change }\end{array}$ & $\begin{array}{c}\text { Sign. F } \\
\text { change }\end{array}$ \\
\hline $\mathbf{1}$ & Accountability & & & & & \\
2 & Training & $.761^{* * *}$ & .484 & .429 & 262.225 & .000 \\
3 & Accountability* Training & .018 & .535 & .0000 & .143 & .705 \\
\hline $\mathbf{1}$ & Autonomy & $.543^{* * *}$ & .200 & .145 & 57.260 & .000 \\
2 & Relationship & $.350^{* * *}$ & .375 & .176 & 88.536 & 000 \\
3 & Autonomy * Training & .048 & .377 & .001 & .707 & .401 \\
\hline $\mathbf{1}$ & Entrepreneurship & $.655^{* * *}$ & .443 & .388 & 219.664 & .000 \\
2 & Relationship & $.207^{* * *}$ & .492 & .050 & 30.727 & .000 \\
3 & Entrepreneurship * Training &. $\mathbf{1 0 0}^{* *}$ &. $\mathbf{4 9 9}$ & $\mathbf{. 0 0 7}$ & $\mathbf{4 . 3 1 9}$ & $\mathbf{. 0 3 9}$ \\
\hline Note: $* \mathrm{P}<0.1 ; * * \mathrm{P}<0.05 ; * * * \mathrm{P}<0.01$ & & & & &
\end{tabular}


The moderating effects of the intervention variables can be plotted in the figures below. As presented in Figure 5-7, when communication is high, the relationship between HR autonomy and employee PM is strengthened. More specifically, with the same score for HR autonomy, the employee PM system tends to be more advanced when the organisation intensifies communication about the employee PM practice.

\section{Figure 5-7: Effect of communication on the HR autonomy-employee PM relationship}

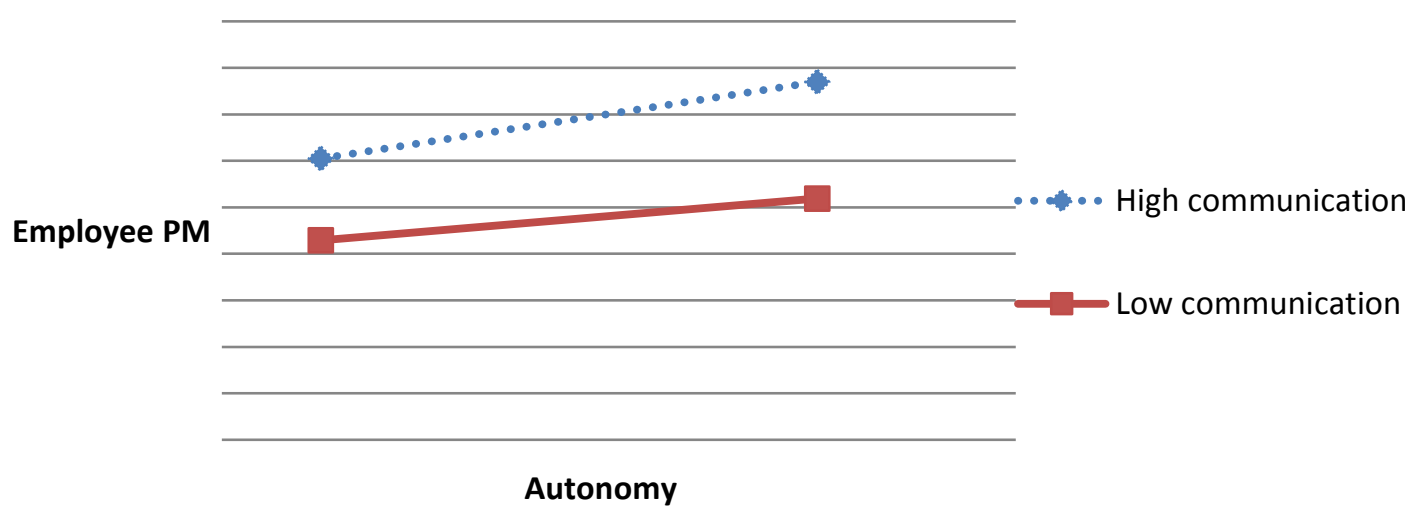

Similarly, the moderation effect of communication on the entrepreneurship - employee PM relationship is plotted in Figure 5-8. The figure shows that at the same level of entrepreneurial leadership, employee PM practice is promoted by strong communication. In other words, the impact of entrepreneurial leadership on employee PM is heightened when communication is high.

Figure 5-8: Effect of communication on the entrepreneurship-employee PM relationship

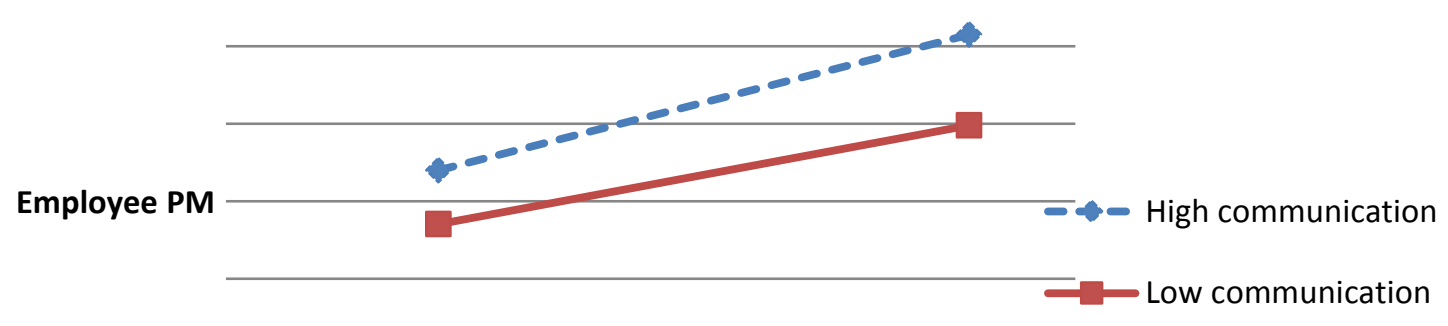

Entrepreneurship 
Finally, Figure 5-9 depicts the effect of training on the entrepreneurial leadershipemployee PM relationship. Accordingly, the impact of entrepreneurial leadership on employee PM is heightened when training is provided.

\section{Figure 5-9: Effect of training on the entrepreneurship-employee PM relationship}

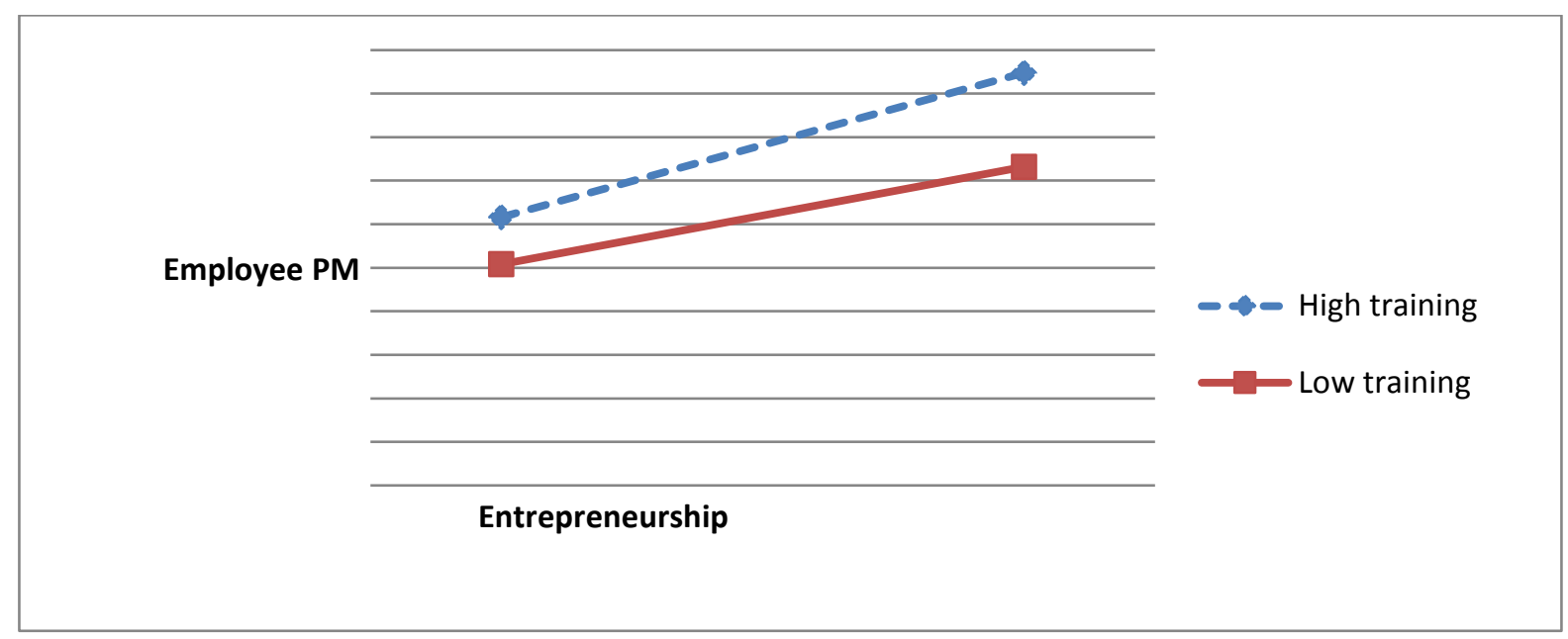

To sum up, communication and training play active roles in strengthening the relationships between the contextual factors and employee PM. Communication and training significantly intensify the effects of HR autonomy and entrepreneurial leadership on the development of employee PM. However, neither communication nor training moderates the relationship between accountability and employee PM.

\subsection{Qualitative analysis}

This section provides insights into how and why the hypothesised relationships arose or did not. More specifically, it provides evidence from the qualitative data analysis to shed light on the following research questions:

1. How and why could or could not employee PM improve the outcomes regarding employee attitudes and organisational performance?

2. How and why could or could not the contextual factors drive the development of employee PM?

3. How and why could or could not the contextual factors directly and indirectly affect the outcomes variables?

4. How and why could or could not the cultural factors moderate the relationships between the contextual factors and the development of employee PM? 
5. How and why could or could not the intervention practices moderate the relationships between the contextual factors and the development of employee PM?

As stated previously, the qualitative data in this research was primarily collected via semistructured interviews, using the interview questions attached in Appendices E, F and G. After being collected and translated, the qualitative data was codified and then grouped into themes. These themes were connected, compared and integrated in various patterns aimed at developing relationships and theories related to the research questions. In addition, some themes emerging in the coding process were also reported such as the effect of corruption on the implementation process of employee PM. The following sections provide evidence under themes for comparing and contrasting these findings with the findings from the quantitative analysis.

\subsubsection{The development of employee PM and the outcome effectiveness}

The impact of introducing the five employee PM practices on employee attitudes and performance was revealed in depth by the interviews. For example, within organisations adopting a suite of employee PM practices, including appraisals based on quantitative outputs, links to pay, participation encouragement and movement of low performers to less demanding positions, the respondents saw these changes positively in contrast to earlier approaches.

The interviews revealed ample evidence of how the advancement of employee PM could improve outcomes in terms of employee attitudes and performance.

\section{The advanced cluster}

A typical example of adopting an advanced PM scheme is rewarding employees based on clearly defined performance standards.

"In our new PM scheme, the performance standard of each job position is clearly identified. The employees' rewards largely depend on their accomplished performance..... consequently, employees' attitude and responsibility have significantly changed, and some employees now asked [for] more tasks in order to have increased rewards including salary bonuses ...."

There is also innovation in giving feedback that overcomes traditional, face-saving barriers. One of the respondents in the advanced cluster explained:

“...The new appraisal system requires cross-rating that allows co-workers to give feedback and rate each other via an anonymous online appraisal software programme. Therefore, feedback and ratings are more objective and accurate because employees do not have to give feedback directly as the traditional method. Besides, the connection between pay and the unit's performance makes colleagues give feedback frankly as well as rate each other accurately. Poor 
performers and lazy employees will be frankly criticised by co-workers because their performance will affect the performance and income of the entire unit..."

The advanced cluster also supports increased employee participation, as indicated by the following comment from a senior manager:

"For the PM practice to be effective, we enable employees to participate in setting goals in order to increase the understanding and acceptance of job goals. Also, we encourage them to discuss how to distribute the supplementary salary fund of their team. This helps the employees to feel their ownership in the implementation, thus increasing job satisfaction.”

As well as revising egalitarian pay distribution mandates and dealing with the problem of inertia in the public sector, the advance cluster also takes drastic measures to address poor performers.

"The majority of public employees still think that a monthly base salary is their vested interest regardless of their performance. This causes stagnation in the public sector. In order to erase this erroneous mindset, we stipulated that employees would only receive full base salaries if they are rated as satisfactory for the entire year. If one employee is rated unsatisfactory for two months, his rating in that entire year will be unsatisfactory and he will not get the full base salary. Besides, if one employee is rated as unsatisfactory for two consecutive years, he will lose his job. To do so, we have developed appraisal criteria that adhere to the completion of assigned tasks. If rating only relies on political quality and ideology, we cannot point out who is better. So far, some employees have had to voluntarily leave the agency because they themselves realised that they could not meet the performance standards."

Although such drastic measures are still rare among public organisations, and the reduction of base salary is not yet regulated by law, they have had substantial effects on the inert attitude inherent among public employees. One chief executive in the advanced cluster proudly said that:

"Since innovating employee PM, the attitude of employees in my organisation has substantially changed. You will not be able to observe employees in my organisation playing games, chatting or complaining about work like in many other public organisations. They all work hard. Even if someone is absent, other co-workers are willing to assume that work because all work fulfilled will be connected to income. If employees accomplish more work, they will receive a greater salary bonus at the end of the month."

\section{The transitional cluster}


In these organisations, frequent appraisal and output measures are effectively used. A report from Danang (one pilot province) indicated that output-based appraisal has considerably improved the accuracy and fairness of the appraisal practice, thereby better distinguishing employees' performance. For instance, the percentage of people who were rated as excellent fell from 21.4 percent to 4.4 after the output-based appraisal scheme was adopted. Meanwhile, the percentage of people who were rated as "accomplishment of tasks with limited capacity" increased from 4.3 percent to 11.4 percent (Danang 2014).

Although the classification of employees was done well, rating results were not meaningfully linked to rewards (including discipline). This caused the adoption of employee PM to become half-hearted in time:

“...Our PM system seems to be very effective because employees are appraised monthly against specific output criteria. Thanks to this appraisal, supervisors can uncover poor performers, thereby adjusting placements promptly. But the supplementary [performance-based] salaries are only 500.000 VND (equivalent 22 USD) per month. Meanwhile, promotion decisions are usually based on personality and political factors, rather than performance and competency.....after a while, employees thought that this amount of money does not justify greater effort..."

The respondents also noted several, often conflicting policies, with gaps between what is espoused and practised by the upper agency. More specifically, half-hearted decentralisation and unsystematic reforms made the implementation of PM less fruitful. One of the consequences is the lack of support needed in order to handle poor performers effectively.

“...In spite of being a self-financing institution, most of our HR practices such as recruitment, remuneration and promotion still need the approval from the upper agencies...for poor performers, the government stipulates that these people are only dismissed if they are rated as being unsatisfactory for two straight years. However, it is very difficult to rate an employee as unsatisfactory because they still meet the government's principal appraisal criteria such as obeying the party's resolutions and laws, maintaining good political quality ..."

Some interviewees also said that institutional problems, particularly the backwardness of the current HRM regulations, prevent the efforts of public organisations from comprehensively reforming employee PM. This is especially true for administration agencies with limited autonomy:

"In my province, we have done everything possible to achieve a robust appraisal system in terms of the technical dimensions such as monthly output-based appraisal, anonymous 
feedback...but the system does not seems to be very effective and fruitful due to the constraints associated with the institution... This is because the system can't answer the key question 'What is the purpose of appraisal for?', when the organisation does not have autonomy to increase salary and promote good performers."

In addition to the above reasons, the moderate development of employee PM is affected by cultural factors and other traditional values. Specifically, although some organisations adopt relatively advanced employee PM practices, the units within the organisations are likely to manipulate these mandates in different ways. This perspective can be illustrated by the following example:

"Based on each division's rating result, the department delegates the head of divisions to decide who should be rated as $\mathrm{A}, \mathrm{B}$ or $\mathrm{C}$ which will then translate into extra income levels. However, some heads of divisions are harmonious persons, afraid of displeasing others and wanting to avoid conflicts with subordinates. As a result, they will compromise with the staff in determining ratings and distributing extra income. Accordingly, the division's additional salary fund will be evenly allocated among all employees in the division. Because the department forces divisions to classify employees, thus, those with higher ratings will have to pay back the difference to the division fund...Meanwhile, other divisions compromise by alternately rating members as B for some months and A for the remaining months, regardless of how well they have performed the job and positions they hold."

\section{The laggard cluster}

The employee PM practice of these organisations mostly relies on the annual appraisal practices where employees are still evaluated on political quality, compliance and honesty. Additionally, the connection between performance and consequences is very limited as well.

“...Our appraisal criteria such as compliance with the party's resolutions, political personality, loyalty and morality are too general and vague. They are not valid and reliable enough to distinguish employee performance fairly. In fact, almost all the employees are rated as having fulfilled assigned duties well. Such a system can't help to motivate good performers, neither does it handle poor performers..."

As a central element of the entire employee PM system, poor appraisal practice in turn bedevils other PM practices, particularly performance-based pay and merit-based promotion. This observation is illustrated by the following examples: 
“...The current regulations state that the promotion practice needs to be based on the performance appraisal results. However, given the performance appraisal is not accurate, valid and objective, this provision is rarely applied."

“...The pay-for-performance is only really useful when the appraisal practice is conducted in a scientific way with reliable appraisal criteria. Otherwise, it will only cause conflicts. This is why we are very reluctant to adopt performance-based pay."

Unclear appraisal criteria, coupled with a high face-saving culture, make the feedback practice ritualistic and useless. One HR manager described this issue as follows.

“...Because appraisal takes place in collective meetings, people tend to praise each other. In such meetings, people mostly vote to select the best people for recognition (that is often reserved for senior managers) rather than point out weaknesses for remedy... In reality, people avoid giving honest feedback and displeasing others in order to avoid conflict and reprisal..."

Besides the specific problems concerning appraisal practice, the underdevelopment of employee PM partially derives from broader rationales, e.g. the career-based system and rigid personnel policies that prevent public organisations from handling poor performers effectively.

"In Vietnam, people prefer to work in the public sector, though the salary level is very low. The reason is that once they have entered one public organisation, it is so difficult to dismiss them. In reality, almost no one has been terminated because of poor performance, unless he or she has violated laws seriously such as being accused of corruption."

The majority of respondents in the laggard cluster admitted that the current appraisal system in their organisation only plays a very limited role in improving employee performance. One HR manager commented:

"From my point of view, the current appraisal system in my agency does not have any effect on improving employee performance....It is actually not effective. The employees still work in their old way because, regardless of their performance, they are still appraised as 'having well fulfilled assigned duties'. Nor do they want to put more effort in order to be appraised as 'having excellently fulfilled assigned duties' because the award is only symbolic and not considerably different in terms of financial value compared to other ordinary people."

To sum up, the qualitative data provided relatively clear evidence of the effect of employee PM on employee attitudes and performance. Indeed, job-based appraisal increases employee responsibility and work motivation compared to the former appraisal systems that mostly rely on abstract criteria. This is particularly true when appraisal results continue to be 
connected to consequences such as bonuses and discipline. Employees feel more motivated when they perceive that their effort and contribution were compensated by extra income.

Meanwhile, handling poor performance in this case can positively affect employees' attitude to work by signifying that public organisations are no longer a safe resort for lazy and incompetent staff. In addition, in advanced systems, feedback can also play an important role in improving employee performance by helping employees perceive their weaknesses. This is because anonymous online appraisal software programmes and team performance-based rewarding systems make co-workers overcome traditional constraints such as face-saving and interpersonal relationships to provide more honest and frank comments. Finally, encouraging employees' participation in the implementation process not only makes PM mandates more practical but also increases employees' feeling of ownership and acceptance.

\subsubsection{How the contextual factors influence the development of employee PM}

This section explains why and how the contextual variables can promote or constrain the development of the PM practices.

\section{Accountability}

The interviews revealed that the root cause of many problems in the public service, including poor employee PM, derives from the institution, particularly the accountability mechanism. Specifically, the individual accountability of chief executives for the organisation's performance is very limited. The majority of public organisations are currently held accountable by their annual reports. Poor performance or incidents are usually attributed to external causes or collective leadership rather than individual responsibility. In practice, very few chief executives are held responsible and demoted due to poor performance. As a result, very few chief executives do their best to improve the organisation's performance. The following quotes provide evidence to support this argument:

“...Currently, we do not have a sound competitive mechanism, so ministers' accountability is very modest. The lack of a top-down, comprehensive accountability system makes chief executives less interested in the organisation's performance as well as employee PM practices."

"In Vietnam, the accountability of public agencies is mostly carried out through self-reports, while there is no monitoring and evaluation of external independent organisations. In reality, if there is any incident, agencies will try to blame external objective causes. Thus, almost no one has been ever demoted because of poor performance. As a result, the current accountability mechanism is not strong enough to force chief executives to pay serious attention to 
organisational performance and employee performance management unless they want to 'polish' their image for promotion."

Therefore, seeking measures to enhance accountability may be seen as a strategic solution to the problem. The following is a quote from one provincial leader who led his province to reform the accountability mechanism by using organisational performance as a key criterion to evaluate the chief executives.

"The weak institution system, coupled with the collective leadership mechanism, has severely limited chief executives' accountability...In order to enhance accountability, we sought permission from the central agencies to carry out a pilot project that evaluates all departmental directors according to the organisation's performance. Once evaluated by clear and specific standards, coupled with corresponding consequences, the chief executives proactively sought solutions to the organisation's identified problems as well as managing employee performance effectively..."

Another respondent from a central agency commented that:

"In general, people (senior managers) know their own problems. However, they are inhibited by cultural factors and the mechanism to handle them. If ministers evaluate their chief executives in a rigorous and objective manner, the chief executive, in turn, will have to manage and evaluate their employees' performance seriously."

\section{HR autonomy}

In addition to accountability, the evidence from the interviews indicates the important role of HR autonomy in the development of employee PM. As mentioned earlier, public organisations are expected to strictly comply with what upper agencies permit. Any innovation without prior approval can result in negative consequences for public managers. In the current context, public organisations can gain HR autonomy in two ways. The first is by becoming a self-financing institution. The current laws stipulate that public service delivery institutions will be granted management autonomy, including HR autonomy, to the extent to which they can finance operational and investment costs. The second way is through pilot schemes that allow the pilot organisations to innovate certain HR practices within a certain period with the permission of the central authorities. The following comment is from one chief executive whose organisation has been assigned as a self-financing institution.

"Since the government enacted the Decree on the self-financing mechanism for public service delivery institutions, we have more autonomy and revenue to introduce pay-forperformance... which in turn enables us to remedy irrationality in the government's current 
regulations....Accordingly, good performers will be recognised and paid what they deserve regardless of their [lack of] seniority..."

In contrast to autonomous institutions, administration agencies with limited autonomy tend to be reluctant to reform their HR practices. An HR manager of one district-level administration agency revealed the following:

"Almost all our HR practices such as recruitment, promotion, discipline and termination are decided by the city people's committee. In fact, my chief executive has very little autonomy, thereby avoiding adopting drastic measures in appraising and addressing poor performers...Therefore, for employee PM practice to be effective, public organisations need to be decentralised to have more autonomy in personnel decisions."

In response to the question, "What is the cause of the stagnation in innovating employee PM practice?" one senior manager answered frankly that:

"The answer lies in the Ministry of Home Affairs (MOHA) that is in charge of managing public employees nationwide. They always urge other central agencies to decentralise but they themselves do not want to decentralise the function of managing public employees because of their own benefits. This is preventing the innovation of HRM practices in public organisations."

This perspective is consistent with comments in a UNDP study in Vietnam that revealed that:

"At present, the Department of Home Affairs in provinces (DOHA) is working in a vacuum with limited authority in HRM. As the economy grows, we want to be able to solve specific issues in our province... As the central civil service management agency, MOHA should focus on HR policy development, leaving operational matters to the sector agencies and localities to decide" (Poon, Hung et al. 2009 p.4).

In the current context, the respondents concur that becoming a self-financing organisation, coupled with HR autonomy, increases the effectiveness of employee managing schemes. Below is one example:

"For self-financing organisations to operate effectively, they need at least 50 percent autonomy, thereby being able to introduce necessary changes. Having a self-financing mechanism and autonomy will make HR practices better, which will in turn increase productivity and the quality of work by better management of employees' performance." 


\section{Entrepreneurial leadership}

In situations of weak accountability and relatively limited HR autonomy, entrepreneurial leadership has emerged as a critical factor in the success of employee PM. The interviews provide specific evidence about the relationship between entrepreneurial leadership and the maturity of employee PM. The following is a comment from one chief executive in the advanced cluster.

"Chief executives play a critical role in innovating PM schemes. Under this sky (the current institution), the chief executives need to take risks or even exceed the vested authority a bit, so that the change is successful. The reason is that several current obsolete policies are preventing the public organisation from operating effectively..."

This observation is verified by another comment.

"...The current institution does not hold chief executives sufficiently accountable for the organisation's performance. Nor does it clearly stipulate that chief executives will be demoted if they do not fulfil the organisation's goals. Therefore, only chief executives who have high dignity and courage commit to renovating the PM practice. This is because the innovation is not imperative while the change process might result in risks and opposition from the employees...In fact, many chief executives tend to avoid displeasing subordinates. This is not only because of cultural factors but also because of the current personnel policy that regulates that the superior authorities will consult employees via a vote of confidence before appointing or reappointing chief executives..."

This view of entrepreneurial leadership is also shared in the comment below.

“...With regard to introducing employee PM, chief executives' enthusiasm and determination are very important. The way to carry out employee PM varies from chief executive to chief executive though they operate under the same institution. Many people only implement employee PM in perfunctory ways to cope with the regulations....For the PM system in my organisation, although there are still many issues, my chief executive always attempts to correct and refine it. In the first period, the supervisors and employees were not familiar with the system and tended to do it perfunctorily. However, thanks to the determination of the chief executive, all issues are being gradually resolved and the system has started working effectively..."

\section{HR unit competence}

Observations from the interviews show that the role of HR units in the innovation of employee PM is relatively ambiguous, although some well-designed PM systems in this study are often related to competent HR staff. According to some respondents, HR units' expertise 
does not really matter because the current regulations require public organisations to strictly comply with the regulations of upper agencies in HR practices. Therefore, HR units' functions are simply administrative. According to some chief executives, the main responsibility of HR units is to ensure that all personnel practices are aligned with the current regulations rather than improving their effectiveness. Therefore, HR units can only play a passive role in the innovation of PM systems. In response to the question, "What is the role of the HR unit in the innovation of PM practices?" the majority of chief executives responded along the lines of the comment below:

"The personnel unit has the role of monitoring and making sure that the PM practices are compatible with the promulgated mandates. They are also responsible for preparing reports to evaluate if the system is good or not."

As such, under the eye of many chief executives, HR units' expertise does not really matter, or at least it is not their priority. This observation is reflected in the comment below:

"The quality of the personnel unit in my organisation has been affected by the process of organisational restructuring. Staff who cannot be placed in any other position within the organisation will be assigned to the personnel division. Thus, they usually do not have a background in human resource management."

The interviews suggest that HR units rarely have an active role in renovating employee PM. Instead, they are rather passive and only do what is directed by chief executives. This is reflected in the comment below.

“...our HR department's competence is quite good. One deputy director of the department even earned a Master degree in HRM from England. However, the personnel practices are supposed to strictly follow the guidance of the upper agencies...Any change in personnel practice is relatively sensitive. Chief executives tend to avoid 'touching' this domain. Hence, only if the chief executive is willing to take risks, can the HR department leverage their role in renovating employee PM ..."

Although by itself, HR competence does not directly drive the development of employee PM, advanced PM systems usually have competent HR units. In these organisations, HR staff help transform chief executives' ideas for improving employee performance into specific policies and mandates. In fact, developing and implementing an effective employee PM is a difficult task, particularly in the public sector. This requires the designers to have not only solid knowledge of modern HRM but also a sufficient understanding of current regulations. Only by doing so can the organisations adopt the changes necessary for an effective PM system while 
ensuring compliance with the current regulations. The following quotation shows that if encouraged and enabled by chief executives, HR units can make a considerable contribution to the effectiveness of employee PM.

“...The role of the personnel unit is dependent on the chief executive's perspective. Our former director always sought solutions to improve the department's performance by improving the employees' performance. The HR units were encouraged and enabled to leverage their competence in seeking solutions aiming to put his ideas into practice. So the HR unit played a very active role in innovating PM practices and other HRM practices. However, when the director retired, the new director was not really interested in improving the department's performance. He only required the HR units to conform to the set procedures. Consequently, the personnel unit has not put forward any proposal to improve the department's performance. The employee PM practice has gradually returned to the old system."

In short, the hypothesis regarding the driving role of the contextual factors is generally supported by the qualitative data strand. Increased accountability, HR autonomy and entrepreneurial leadership are perceived as antecedents or drivers for renovating employee PM practices. This is because they promote or enable public organisations to overcome impediments existing in the current public service such as bureaucracy, high administrative hierarchy, weak accountability and enduring inertia. As well as these three factors, HR units' competence can facilitate the development of employee PM, but they can maximise its potential only if encouraged and enabled by chief executives.

\subsubsection{How the contextual variables affect the outcome variables}

The two sections above provide a lot of vivid evidence about how the contextual variables drive the development of employee PM, which in turn induce improved employee attitudes and organisational performance. In other words, the interviews provide an insight into the reasons why the contextual variables can indirectly influence employee attitudes and organisational performance through the mediator of employee PM.

For direct effects, although the research's initial purposes did not aim to explore such relationships, the qualitative data provides some evidence about how these processes could arise. The following comment from a provincial leader shows how increased accountability (by evaluating organisational performance), can improve employee attitudes:

"The pilot scheme of evaluating and ranking organisational performance has created an emulation movement among the organisations within the province. The reason is that the ranking results are publicly announced. Well-fulfilled organisations are recognised while poor 
performing organisation are criticised, even disciplined. As a result, not only chief executives but also employees have to attempt together to fulfil assigned goals if they do not want their organisation classified as a low-ranking organisation. Doing so has promoted democratic working style and solidarity within the organisation, thereby leveraging the strength and creativity of each individual and team to fulfil mutual goals."

Apart from accountability, HR autonomy can also directly affect employee attitudes and organisational performance in various ways. For example, HR autonomy usually accompanies the self-financing mechanism that means the organisations no longer receive a state budget as in the past and have to operate as an enterprise. Accordingly, employees' income and even jobs depend on the organisation's performance. This makes both executives and employees pay more attention to the organisation's performance and efficiency:

"Having become a self-financing institution, we ourselves have to pay all operational costs. Also, we have to compete with other service-providing institutions and private companies to generate revenue. Thus, both managers and employees have to change work attitudes to maintain the organisation's survival, as well as increasing income."

Additionally, the self-financing mechanism increases the flexibility of decisions in investment, expenditure and management, which in turn improve productivity and operational efficiency:

"The self-financing mechanism and increased autonomy enable public organisations to have more discretion, flexibility, as well as responsibility in fulfilling their duties. Therefore, they can make more reasonable and timely decision, thereby increasing productivity and performance."

Similarly, the interviews indicate that entrepreneurial leadership can affect employee attitudes and organisational performance in different ways. For example, the leaders with entrepreneurial leadership styles usually win high credibility and respect from employees because they are willing to sacrifice their personal interests for the organisation's. In addition, they are persistent and decisive in pursuing initiatives aiming to improve the organisation's efficiency. The following comment is from one chief executive whose organisation was evaluated as very successful in performing its mission as well as developing an employee PM:

"Renovating employee PM is only one of many reform activities aiming to help the organisation achieve its strategic goals...I always regard the PM innovation scheme as my spiritual offspring. I have tried my best so that it does not fail. Also, I have had to be very persistent, determined and even sacrificed my own interest in order to become a good example for other managers. If the senior manager can't set a good example, reform initiatives will not 
be successful...In the beginning, many people, even some managers opposed reform, due to difficulties. After 10 years of continuous improvement, people have seen its effectiveness. In fact, many public organisations also want to adopt such a scheme to improve the shortcomings of the existing system. However, most of them decide to discontinue it because they are unable to overcome initial obstacles."

The interviewees revealed that exemplary chief executives can be a good motivator for their employees. The following is one example:

"Our former executive is an exemplary leader who had a good vision, integrity and responsibility to perform the organisation's duties. We really respected him and always worked with a strong sense of responsibility and honour. In fact, we always tried our best to study and work to fulfil tasks at the highest level. Therefore, it is critical to develop exemplary leaders. Otherwise, all resolutions and solutions for improving performance in the public sector will only have limited impact."

In sum, the contextual factors have not only indirect but also direct effects on employee attitudes and organisational performance. Consistent with the quantitative analysis, the qualitative analysis prove that, in addition to the employee PM channel, the contextual factors could affect the outcome variables in different ways. This finding implies that while employee PM is important, there are also other mechanisms to improve employee attitudes and organisational performance. Once again, this confirms the importance of the contextual factors in improving employee attitudes and organisational performance.

\subsubsection{How the cultural factors affect employee PM}

This section provides evidence from the interviews regarding the effect of the cultural factors (i.e. nepotism and personal relationships) on the relationships between the contextual factors and the employee PM practice.

\section{Nepotism}

Nepotism has become a prevalent issue in the contemporary public service in Vietnam. Almost all the respondents acknowledged that it exists in their organisations, albeit at different levels. The proverb "When a person becomes a mandarin, the entire family lineage may benefit" is rooted in the perception of many Vietnamese people. Mass media has repetitively warned about the recruitment and promotion of leaders' relatives in public organisations. This issue has become so common that the incumbent Prime Minister Nguyen Xuan Phuc has ironically said that "we need to look for talented people, not for relatives" (Government 2016). Nepotism not only prevents public organisations from recruiting competent employees but also reduces the 
effectiveness of employee PM innovation in various ways. This observation is illustrated by the following examples:

"Given nepotism, it is difficult for placement and performance management. This is because the supervisors tend to favour the leaders' relatives in placing, rewarding as well as addressing poor performance. For example, instead of prioritising competent staff, training overseas usually targets the leaders' relatives although they are not deserving and their job position does not require such training programmes."

"Feedback and appraisal are heavily influenced by the culture of face-saving, personal relationships and nepotism. My organisation has a retired leader's son who only comes to the office twice a week. Despite being reminded several times, he is still regularly absent. However, at the end of the year, he is still rated as satisfactory fulfilment."

Nepotism not only affects feedback and rewarding but also steadily nullifies the practice of handling poor performers. The following honest comment illustrates this issue:

"In my organisation, many people are relatives of senior leaders. Therefore, when implementing appraisal activities, I usually have to pay special attention to kinship relationships before making decisions, particularly in handling poor performers. Consequently, such behaviours might distort the entire PM system, because if I rule one employee with a light touch, I will have to do the same with others."

Consistent with this observation, another senior manager admitted that:

"As a vice-rector, I really want to conduct our appraisal practice accurately and objectively, so that we can improve the university's operational efficiency by placing the right people into right positions. However, I myself and even the rector sometimes can't do so because many people are recruited, placed and promoted because they are relatives of leaders at higher-level agencies. It is so difficult to transfer poor performers to other less-demanding positions. In some cases, you have not yet transferred these persons (poor performers), their uncles have already transferred you to other positions."

The interviews revealed that nepotism not only undermines existing PM systems but also prevents public organisations from renovating the current PM systems.

“...Many people who were admitted through kinship relationships only seek a stable job in the public sector. Therefore, their competence, work motivation and performance are rather limited. If the appraisal practice is rigorously conducted, these people will be impacted firstly. Therefore, they tend to oppose any innovation regarding employee PM..." 


\section{Personal relationships}

It has been widely said that the personnel practices in the public sector in Vietnam are often affected by nepotism, bribery and personal relationships (Hoang 2014). There is a great deal of evidence about the negative effects of this cultural factor. The following is some evidence of the effect of personal relationships on employee PM:

"The implementation of goal-based appraisal in the public sector is very difficult because many people do not currently meet their job specification. This stems from poor recruitment in previous years that was usually dominated by personal relationships rather than merit."

In fact, it is not too difficult to identify poor performers, but it is not easy to handle these people effectively in a strong personal relationship culture. One senior manager said that:

"In public organisations, maintaining personal relationships is crucial. When entering the public sector, I have had to adjust my own behaviours to harmonise relationships and avoid conflicts...for example, handling poor performers drastically can displease many people in the organisation. Consequently, my career, promotion and interest are likely to be likely adversely affected."

The following is a common view shared by many respondents.

"In reality, if two persons have the same competence, the person who has better relationships and better family background will certainly be more highly favoured in the promotion process."

The personal relationship culture also negatively affects other employee PM practices such as feedback because maintaining good relationships is crucial for almost everyone. This is illustrated by the following observations.

"In appraisal meetings, instead of focussing on performance, people usually pay attention to who the appraisee is. In fact, those who pay attention to 'foster' personal relationships usually receive positive feedback and good appraisal results."

"Vietnamese people have a proverb: 'a good medicine tastes bitter, a true statement displeases the listener'. So straight feedback can lead to a change in relationships or even hostility. Therefore, for many people, providing honest feedback does not return any personal benefit, but can cause losses..."

In addition, the interviews revealed that interpersonal relationships not only damage current employee PM practices but also prevent public organisations from refining this practice:

"In fact, many senior managers do not want to have a rigorous employee PM system because this means that they will have less flexibility to promote the people they prefer." 
The interviewees' evidence shows that the adverse effect of personal relationships is exacerbated by obsolete policies that rely on the consensus principle and collective leadership in the process of decision-making. For example, one chief executive said that.

"Given the collective leadership mechanism, and sometimes inordinate democracy, many managerial issues must be discussed and get consent from all employees in the collective i.e. from the chief executive to the cleaner. This makes the managers avoid displeasing others, even subordinates, which in turn prevents supervisors from commenting honestly and rating objectively....For example, some HR practices such as promotion and recognition need consensus from all employees in the collective. Therefore, those who usually comment frankly may miss out on career development opportunities because they do not get enough support or votes necessary from the collective."

However, the interview findings show that the personal relationship culture can be changed or moderated by chief executives' determination to hold supervisors accountable for the accuracy of ratings:

"In the initial period, supervisors tended to rate perfunctorily and ignore employees' errors. However, under pressure from the chief executive, the supervisors who did not appraise accurately and objectively were punished by having their salary deducted on the grounds that they had not fulfilled their managerial role as a fair and objective appraiser. Consequently, the supervisors had to invest time to rate accurately and fairly. Consequently, the negative effects of harmonious culture, sentimentalism, and face-saving have been considerably curbed."

In summary, apart from the institutional and capacity factors, the cultural factors of nepotism and interpersonal relationships cannot be overlooked in the implementation of employee PM. The evidence from the interviews shows that these cultural features lead to a deterioration in employee PM practices from goal-based appraisal, rewarding and feedback to handling poor performance. However, these issues can be mitigated by developing objective performance standards and holding supervisors responsible for appraisal results.

\subsubsection{How intervention practices strengthen employee PM}

While the cultural factors can weaken the development and effectiveness of employee $\mathrm{PM}$, the interviews also suggest that communication and training can promote the effectiveness of employee PM. 


\section{Communication}

Evidence from the interviews shows that the success of employee PM is often associated with effective communication activities. Communication is seen as a useful means to make the innovation process more fruitful. The following is a comment from one chief executive:

"Innovating employee PM is like a change process. Therefore, we need to propagandise and explain it to employees so that they can understand and support this innovation process. For example, we communicated to employees that having become a self-financing institution, we ourselves would have to pay all operational costs, including salary and fringe benefits. Therefore, the performance management scheme was indispensable to improve the product's quality and reduce its cost to compete with other institutions in tendering projects, thereby increasing income for the employees."

For the communication practice to be effective, it has to be undertaken in democratic ways. Simultaneously, it should enable employees to understand innovation is important not only for the organisation but also for themselves. The following quotations describe this observation:

"For the scheme to be effective, we encouraged two-way communication that allows employees to openly and democratically discuss the content of the scheme, particularly things that relate to them... The scheme only works well if it ensures the harmony and interests of all parties. In fact, in the initial period, employees might be less concerned about how much they are paid than about transparency and fairness."

"When the appraisal innovating scheme was first introduced, it faced several difficulties and even opposition. To overcome these challenges, we propagandised the aims of the scheme in order to get involvement and support from all parties. It is also necessary to make supervisors understand that the appraisal innovating scheme is a useful tool to serve their managerial tasks."

'Employee PM is a new management practice. So it is necessary to raise employees' awareness of the necessity, the essence, the goal, and procedures of the new PM practice, thereby helping them to perceive their own responsibility in participating in this process proactively and democratically."

\section{Training}

Undoubtedly, a well-designed PM system is important. However, its effectiveness might be limited if the users do not really understand and master it. The interviewees pointed out that training can effectively support the innovation of employee PM. The following is one example: 
"In the initial period, because appraisal activities were time-consuming, supervisors and employees were unfamiliar and very reluctant to implement them sufficiently. Therefore, we organised some training courses for all supervisors and employees to familiarise them with the new appraisal system. These training courses helped the employees better understand and then accept new PM practices, which in turn made the employee PM system operate smoothly."

Organisations in the advanced cluster tend to provide more such training courses for employees. As one interviewee from this cluster said:

"We have conducted several workshops to ensure that employees understand and implement employee PM correctly."

Organising training courses not only helps employees understand the goals and regulations of the new PM practice but also enables them to voice their concerns, thus increasing acceptance of the practice. For example, one chief executive commented:

"Thanks to the training courses, the employees have a better understanding of the procedure of appraisal and the formula of pay-for-performance. This, in turn, increases the transparency of the implementation process. In addition, the training courses enable employees to voice their concerns in formulating and amending the employee PM regulations."

In summary, despite not being seen as antecedents driving the innovation of employee PM, communication and training can reinforce the effectiveness of the employee PM practices. Evidence from the interviews suggests that for communication to be effective, it has to be an open, democratic and two-way process. It is critical to allow employees to voice their concerns as well. Meanwhile, for training courses to be useful, they need to help employees master implementation procedures as well as enabling the organisations to understand employee perceptions, thereby discovering and correcting shortcomings promptly.

\subsubsection{Other findings from qualitative data analysis}

As well as backing up the quantitative findings, the qualitative data shows that the development of employee PM is likely to be influenced by other factors apart from the hypothesised ones. For example, administration agencies whose main responsibilities are developing and enforcing policies tend to have less developed employee PM systems. This is not only because they often have less autonomy but also because of their job features such as ambiguity, instability and dependence on factors outside their control. These combined features make it more difficult to develop individual goals and evaluate employees accurately. With regard to this issue, one middle manager in a central agency said: 
"For administration agencies, outputs of employees are policies and legal documents. Some kinds of documents can be accomplished in some weeks but others can need a whole year. However, evaluating the quality of such outputs is very difficult because the objects impacted by policies are usually very large and it can take $3-5$ years or longer to see the impact and consequences. Additionally, final products (policies) usually involve many management levels and many departments. The delay of accomplishing a legal document is most likely to be out of the control of the specialist"

Furthermore, the respondents also highlighted the effect of corruption on the introduction and implementation of employee PM. This following are some of quotations cited from the interviews.

“...chief executives in many public organisations form 'interest groups' to look after their own interest. Employees who raise voice for the organisation's general benefit or fight against destructive behaviours will be isolated. As a result, many public employees constrain themselves from commenting or participating in the organisation's affairs. They only attempt to perform their jobs at minimum requirement. That is why, regardless of the government' reform effort, the performance of public organisations is still limited..."

Besides, corruption can undermine merit principles in other ways, as the following comment from a researcher:

"In theory, the self-financing mechanism is to force public service delivery organisations to improve operational efficiency and productivity so that they can compete to win contracts and projects. However, the government's tendering research projects, particularly in provinces, are still dominated by the 'ask and give mechanism'. Eighty percent of our contracts are based on relationships. If you are simply researchers, you can't win contracts or projects. With regard to research projects in some provinces, in order to win contracts, you will have to cut back by 30 40 percent of the contract's value. This makes the performance appraisal and HRM activities less important because revenue is mostly generated through personal relationships rather than research competence and quality."

Apart from the effect of job types and corruption, the qualitative analysis from secondary data revealed that although employee PM is an effective tool to improve employee attitudes and organisational performance, it can lead to unwanted consequences. For example, to create funds for performance-based pay, some hospitals sought ways to increase revenue such as requiring patients to buy unnecessary medical services (MOH 2018). Meanwhile, to save administration costs in order to fund performance-based pay, some administration agencies reduced fieldwork 
related to information collection. Although this can benefit employees, it can deteriorate the quality of public policies because of the lack of input of information in decision-making.

\subsection{Integrating the quantitative and qualitative findings}

The results from the qualitative and quantitative analyses indicate that overall, the quantitative and qualitative data converge in answering the research questions, albeit with some differences. The following will discuss the extent to which the qualitative findings agreed with the quantitative findings in examining the hypotheses:

Hypothesis 1: Employee PM is associated with increased perceptions of employee motivation (H1a), organisational commitment (H1b), job satisfaction $(\mathrm{H} 1 \mathrm{c})$ and organisational performance (H1d).

The quantitative findings from the cluster analysis and one-way ANOVA show that the development of employee PM is positively associated with the desirable outcomes. Specifically, the advanced cluster has the highest average scores for motivation, job satisfaction, commitment and organisational performance. Meanwhile, the laggard cluster has the lowest average scores for these outcomes. This finding is illustrated and explained by a lot of evidence from the interviews. For example, given the objectivity, goal-based appraisal helps increase justice in income distribution and personnel decisions, thereby increasing job satisfaction and organisational commitment. Meanwhile, performance-based reward enables employees to receive bonuses or extra income based on their efforts and contribution, which in turn increase working motivation and productivity. Similarly, innovation in feedback helps employees receive more honest and frank comments to recognise their weaknesses that need correction and improvement. Additionally, addressing poor performance helps organisations remove a source of inequality and inertia, thereby increasing job satisfaction, motivation and organisational performance.

The qualitative research reveals that goal-based appraisal can increase employee responsibility and motivation, but only to a limited extent if it is not connected with performance-based rewards. Similarly, pay for performance, feedback and addressing poor performance tend to be ineffective if they do not derive from robust appraisals that are based on jobs or goals.

Hypothesis 2: Agency accountability (H2a), HR autonomy (H2b), entrepreneurial leadership (H2c) and HR competence (H2d) are associated with the development of employee PM practices. 
The quantitative findings show that agency accountability, HR autonomy and entrepreneurial leadership drive the development of employee PM. Meanwhile, HR competence can only affect employee PM if entrepreneurial leadership is present. The qualitative data helps explain the role of agency accountability, HR autonomy and entrepreneurial leadership in the development of employee PM. Also, it explains why HR competence itself is insufficient to promote such development.

Accordingly, holding chief executives accountable for organisational performance forces them to pay more attention to measures aiming to increase productivity and work efficiency. As a result, they have to change their management modes, including methods to manage employee performance. Similarly, increased HR autonomy gives the organisations confidence to remove irrationality in existing regulations as well as adopting more effective employee management practices. Along with accountability and HR autonomy, many respondents mentioned the role of entrepreneurial leadership in the process of innovation. Specifically, facing conditions of bureaucratic culture, limited autonomy and weak accountability, only chief executives with vision, innovative spirit, and courage are willing to take risks to introduce reform initiatives for the benefit of the organisation and public interest.

Although there is evidence associating HR competence with the development of employee PM practices, HR competence by itself cannot drive or lead the innovation. Because of the central management mechanism in the personnel practice, HR units can only leverage their potential in the innovation process with the support of chief executives. This finding corresponds with the result of the multinomial logistic analysis that reveals while HR competence is not significant in predicting the development of employee PM, the interaction term between HR competence and entrepreneurial leadership is significant.

Hypothesis 3: Agency accountability (H3a), HR autonomy (H3b), entrepreneurial leadership (H3c) and HR competence (H3d) indirectly affect employee motivation, organisational commitment, job satisfaction and organisational performance through the mediation role of employee PM.

In essence, the findings regarding this hypothesis are an extension of the findings from the two preceding hypotheses. As discussed above, the qualitative data provides ample evidence of why and how accountability, HR autonomy and entrepreneurial leadership drive the development of employee PM. This development in turn improves employee motivation, organisational commitment, job satisfaction and organisational performance. Consistent with 
this qualitative evidence, the results from the SEM technique indicate that the indirective effects of accountability, HR autonomy and entrepreneurial leadership on all the four outcome variables are significant $\left(\mathrm{P}_{\text {value }}<0.05\right)$.

In addition to confirming the indirect effects, the results from the SEM reveal that direct affects also occur. As such, along with employee PM, the contextual factors can influence employee attitudes and organisational performance through other channels. Although these findings are unexpected, the qualitative data can provide some clues. For example, evaluating and ranking organisations can enhance self-perception and ego of employees, thereby promoting emulation movements between units. Meanwhile, chief executives with integrity, an innovative spirit, vision and willingness to sacrifice their personal interest for the public interest can win the respect and admiration of their followers, which in turn reinforces employees' work morale and performance.

Hypothesis 4: The relationships between the contextual factors and employee PM are moderated (weakened) by the cultural variables of nepotism (H4a), and interpersonal relationships $(\mathrm{H} 4 \mathrm{~b})$.

The results from the quantitative analysis indicate that interpersonal relationships and nepotism significantly moderate the effect of HR autonomy and entrepreneurial leadership on the development of employee PM. However, unexpectedly, neither of them moderates the effect of accountability on employee PM.

As presented above, the qualitative data provide ample evidence that interpersonal relationships and nepotism negatively affect the development of employee PM. However, the explanations of moderation mechanisms are not usually easy (Andersson, Cuervo-Cazurra et al. 2014). In this case, it is even more difficult to explain why nepotism and interpersonal relationships can moderate the effect of HR autonomy and entrepreneurial leadership, but not accountability. However, the following are some likely explanations.

HR autonomy and entrepreneurial leadership do not usually accompany holding supervisors or line managers accountable for conducting these practices. Thus, although an organisation may have introduced a well-designed employee PM system, its supervisors can implement or manipulate these practices in their own ways. For example, some respondents reported that increased autonomy and the innovative spirit of chief executives led to the adoption of goal-based appraisal and pay-for-performance for the entire organisation. However, in some units or divisions where high levels of interpersonal relationships or nepotism were 
present, the heads of units still sought ways to replace pay-for-performance with equal or egalitarian distribution, aiming to avoid conflict between members. In addition, to increase the effectiveness of feedback, some organisations introduced anonymous online cross-feedback software. However, individual relationships still affected the ways the people provided feedback and conducted evaluations.

Meanwhile, increased accountability forces chief executives to devise the employee PM practices in order to meet upper agencies' set goals as well as hold lower-level managers accountable. As presented above, holding supervisors accountable for the unit's performance and appraisal results forces them to take the employee PM practices seriously because they will be punished if they rate and reward employees perfunctorily. Accordingly, interpersonal relationships and nepotism can moderate the effects of HR autonomy and entrepreneurial leadership on employee PM, but may not significantly moderate the effect of accountability on employee PM.

Hypothesis 5: The relationships between the contextual factors and employee PM are moderated (strengthened) by communication (H5a), and training (H5b).

The qualitative and quantitative analyses indicate that communication and training play a positive role in promoting the development of employee PM. Besides the significant and positive associations with the development of employee PM, as reported in Table 5-11, these two practices strengthen the HR autonomy-employee PM relationship and the entrepreneurial leadership-employee PM relationship. The qualitative data explains that these processes occur because communication enables employees to understand the need and benefits of the innovation. Meanwhile, training helps the users acquire the knowledge and skills necessary to implement new employee PM practices effectively.

However, like the cultural variables, the quantitative analysis reveals that these two intervention practices do not moderate the accountability - employee PM relationship. While the qualitative data do not provide intuitive explanations for this finding, we can assume that accountability is likely to have a relatively strong effect on the development of employee PM. More specifically, increased accountability creates a strong mechanism to promote improvement of organisational performance by requiring supervisors and employees to comply with the employee PM practices closely whether they support them or not.

Although the findings regarding the effects of the moderators on agency accountability are unexpected, they elicit an important implication that accountability may be a relatively 
sustainable mechanism in promoting the development of employee PM as well as organisational performance. The findings show that the effect of accountability is stable even in an unfavourable environment such as a high level of interpersonal relationships and nepotism, and users' lack of knowledge of and support for employee PM.

\subsection{Summary of Chapter 5}

Chapter 5 reports the results of examining the five hypotheses. The first section presents the quantitative analysis results while the second provides evidence and explanations from the qualitative data. In general, the quantitative and qualitative data converge to support the hypotheses. Specifically, the results of the two analysis strands support hypotheses 1, 2 and 3, and partially support hypotheses 4 and 5. Accordingly, the introduction of advanced employee PM practices, in combination, can be beneficial to the organisation in terms of increased employee motivation, organisational commitment, job satisfaction and subsequently organisational performance. Meanwhile, the development of employee PM is driven by accountability, HR autonomy and entrepreneurial leadership and partially by HR competence (given the presence of entrepreneurial leadership). Both data strands also reveal that these contextual factors have not only indirect effects, as hypothesised, but also direct effects on employee attitudes and organisational performance.

In examining the moderating role of the cultural variables and the intervention variables (hypotheses 4 and 5), the results indicate that the HR autonomy-employee PM relationship and entrepreneurial leadership-employee PM relationship are weakened by the cultural variables (i.e. nepotism and personal relationships) while being strengthened by the intervention variables (i.e. communication and training). Interestingly and unexpectedly, none of these variables moderates the accountability-employee PM relationship. Although the qualitative data does not provide intuitive evidence to support this unexpected finding, it provides some clues to explain it. From this unexpected finding, we can get a more profound insight into the importance of the different contextual variables in promoting the development of employee PM. The next chapter is dedicated to discussing the findings in relation to the theories and the existing literature. 


\section{CHAPTER 6. DISCUSSION, IMPLICATIONS AND CONCLUSIONS}

This chapter aims to discuss the findings and relevant implications. It first summarises the research's context, questions and main findings. Then it discusses the findings in relation to the theories and existing literature before moving to contributions and implications for policymakers, practitioners and researchers. The last sections are dedicated to discuss research limitations, recommendations for future research and conclusion.

\subsection{Summary of the research findings}

Personnel issues are increasingly recognised as critical in the public administration of developing countries (Tong, Straussman et al. 1999; Jacobs 2004; Teclemichael Tessema and Soeters 2006; Poon, Hung et al. 2009; Berman 2015). Improving employee and organisational performance has become an essential requirement to increase governments' operational efficiency and intensify national competitiveness in the globalised context (Kim 2002; Arnaboldi, Lapsley et al. 2015). For developing countries, this need is even more urgent when several socio-economic development programmes and PAR programmes are facing the risk of failure or low effectiveness due to the irresponsibility, corruption, poor performance and low work ethics of public employees (Taylor 1992; Randma-Liiv 2005; Berman, Bowman et al. 2015; Ho and Im 2015). Inspired by NPM and underpinned by management theories, employee PM has increasingly drawn interest from policy-makers and practitioners in addressing these problems. In theory, employee PM is supposed to improve performance, increase accountability, control corruption and finally enhance efficiency and effectiveness of public service delivery (Lah and Perry 2008; Goh 2012; Haines III and St-Onge 2012; Kong, Kim et al. 2013; Ohemeng, Amoako-Asiedu et al. 2018).

Unfortunately, the literature has reported numerous failures of public organisations in developing countries when seeking to introduce an employee PM system (Randma-Liiv 2005; De Waal 2007; Liu and Dong 2012; Ohemeng, Zakari et al. 2015). Also, it does not always work well in developed countries where unsuccessful cases are prevalent (Holloway, de Waal et al. 2009). It has been said that, despite the pervasive use of employee PM and no shortage of research in this field, we still do not sufficiently know about the factors and practices that are conducive to an effective employee PM system (Pulakos and O'Leary 2011; Davis 2015). Public organisations in developing countries not only share challenges with their counterparts in developed countries such as conflicting goals, limited budgets and the need for pay 
transparency (Perry, Engbers et al. 2009) but also face challenges regarding incompatible culture, weak institutions and limited capacity (Mendonca and Kanungo 1996; Liu and Dong 2012). This has resulted in suspicion about the application of employee PM in the developing context (De Waal 2007). Regretfully, research on employee PM in developing countries, particularly in the public sector, remains sparse. Departing from the practical problems above and the research gap, this research was conducted to answer the five following questions.

Question 1: Does employee PM in developing countries enhance desirable outcomes such as employee motivation, organisational commitment, job satisfaction and organisational performance?

The formation of this question derives from the fact that unsuccessful PM schemes are very common around the world. There is also a debate about whether employee PM is applicable in developing countries (De Waal 2007). Therefore, this inquiry was carried out for two purposes: 1) to provide empirical evidence to answer whether or not employee PM can be effectively conducted in these countries; 2) if so, to explore the design of an advanced or effective PM system.

Question 2: Which contextual factors drive the development of employee PM?

Once the first question was answered, the research continued to explore the contextual factors driving the development of employee PM. This investigation was to respond to the call for researchers to pay more attention to contextual factors. To date, more and more scholars, e.g. Murphy and Cleveland (1995) and Haines III and St-Onge (2012) believe that contextual factors can affect the success or failure of employee PM. Based on reviewing the literature in developing countries, this research focuses on three institutional factors, namely agency accountability, HR autonomy and entrepreneurial leadership and one capacity factor, namely HR competence.

Question 3: Can employee PM be a mediating factor for the relationships between contextual factors and desirable outcomes?

This research question was developed based on questions 1 and 2. Accordingly, if the contextual factors drive the development of employee PM and employee PM in turn improves desired outcomes, we can predict that the context can indirectly affect the desired outcomes through the mediation role of employee PM. The results from this examination give us a better understanding of the role of the contextual factors in improving employee attitudes and organisational performance. 
Question 4: How do cultural variables i.e. interpersonal relationships and nepotism hinder the development of employee PM?

Apart from the institutional and capacity factors, more and more research, e.g. Aycan (2005) and Ohemeng (2009) has pointed to the role of organisational culture in the implementation of employee PM. For developing countries, traditional culture has been identified as one of the key issues that have impeded and continue to impede the development and effectiveness of employee PM. This research contributes to the literature by investigating two cultural dimensions i.e. interpersonal relationships and nepotism. Though these are rather prevalent in many developing countries, they have not been sufficiently researched in the literature. This research hypothesised that the existence of these two cultural dimensions weakens the relationships between the contextual factors and the development of employee PM.

Question 5: What intervention practices can be introduced to strengthen the development of employee PM?

In this inquiry, this research wanted to explore the intervention practices that organisations can embrace to strengthen the effectiveness of employee PM. There are arguments that the limited success of employee PM systems may be partially attributed to the absence of effective intervention practices (Lawler, Benson et al. 2012). Accordingly, communication and training were investigated under the hypothesis that they will strengthen the relationships between the contextual factors and the development of employee PM.

A mixed methods approach was used to answer these research questions. The hypotheses were examined using a sample of 322 public employees working in 29 diverse organisations in the Vietnamese public sector. Qualitative data derived from 30 semi-structured interviews were used to explain rationales and patterns behind the relationships. The results of the quantitative and qualitative analysis are summarised in Table 6-1 below.

Table 6-1: Summary of the research findings

\begin{tabular}{|l|c|}
\hline \multicolumn{1}{|c|}{ Hypotheses } & Results of analysis \\
\hline Hypothesis 1 & Accepted \\
\hline $\begin{array}{l}\text { H1a: Employee PM is associated with increased perception of } \\
\text { employee motivation }\end{array}$ & Accepted \\
\hline $\begin{array}{l}\text { H1b: Employee PM is associated with increased perception of } \\
\text { organisational commitment }\end{array}$ & Accepted \\
\hline $\begin{array}{l}\text { H1c: Employee PM is associated with increased perception of } \\
\text { job satisfaction. }\end{array}$ & Accepted \\
\hline $\begin{array}{l}\text { H1d: Employee PM is associated with increased perception of } \\
\text { organisational performance }\end{array}$ & \\
\hline
\end{tabular}




\begin{tabular}{|c|c|}
\hline Hypothesis 2 & \\
\hline $\begin{array}{l}\text { H2a: Agency accountability is associated with the } \\
\text { development of employee PM }\end{array}$ & Accepted \\
\hline $\begin{array}{l}\text { H2b: HR autonomy is associated with the development of } \\
\text { employee PM }\end{array}$ & Accepted \\
\hline $\begin{array}{l}\text { H2c: Entrepreneurial leadership is associated with the } \\
\text { development of employee PM }\end{array}$ & Accepted \\
\hline $\begin{array}{l}\text { H2d: HR autonomy is associated with the development of } \\
\text { employee PM }\end{array}$ & Partially accepted \\
\hline \multicolumn{2}{|l|}{ Hypothesis 3} \\
\hline $\begin{array}{l}\text { H3a: Agency accountability (H3a) indirectly affects the } \\
\text { desirable outcomes }\end{array}$ & Accepted \\
\hline $\begin{array}{l}\text { H3b: HR autonomy (H3b) indirectly affects the desirable } \\
\text { outcomes }\end{array}$ & Accepted \\
\hline $\begin{array}{l}\text { H3c: Entrepreneurial leadership }(\mathrm{H} 3 \mathrm{c}) \text { indirectly affects the } \\
\text { desirable outcomes }\end{array}$ & Accepted \\
\hline \multicolumn{2}{|l|}{ Hypothesis 4} \\
\hline \multicolumn{2}{|l|}{ H4a: Personal relationships weaken the relationship between } \\
\hline - Agency accountability-employee PM & Rejected \\
\hline - HR autonomy-employee PM & Rejected \\
\hline - Entrepreneurial leadership-employee PM & Accepted \\
\hline \multicolumn{2}{|l|}{ H4b: Nepotism weakens the relationship between } \\
\hline - Agency accountability-employee PM & Rejected \\
\hline - HR autonomy-employee PM & Accepted \\
\hline - Entrepreneurial leadership-employee PM & Accepted \\
\hline \multicolumn{2}{|l|}{ Hypothesis 5} \\
\hline \multicolumn{2}{|l|}{ H5a: Communication strengthens the relationship between } \\
\hline - Agency accountability-employee PM & Rejected \\
\hline - HR autonomy-employee PM & Accepted \\
\hline - Entrepreneurial leadership-employee PM & Accepted \\
\hline \multicolumn{2}{|l|}{ H5b: Training strengthens the relationship between } \\
\hline - Agency accountability-employee PM & Rejected \\
\hline - HR autonomy-employee PM & Rejected \\
\hline - Entrepreneurial leadership-employee PM & Accepted \\
\hline
\end{tabular}

\subsection{Discussion of the findings}

\subsubsection{Findings regarding research question 1}

Regarding this question, the research finds that public organisations in developing countries can benefit from introducing the five employee PM practices. Specifically, the introduction of the five PM practices, namely: goal-based appraisal, feedback, reward-forperformance, addressing poor performers and employee participation, in concert, can improve employee attitudes and perceived organisational performance. These findings are discussed in detail below. 
First, this research provides empirical evidence to substantiate the applicability of employee PM in the public sector (Verbeeten 2008), particularly in developing contexts (De Waal 2007; Ohemeng, Amoako-Asiedu et al. 2018). This is meaningful because there is suspicion about the effectiveness of employee PM in the developing context. However, the literature lacks empirical research into the effect of PM practices on employee-level outcomes and organisational performance, particularly in the public sector (Donahue, Selden et al. 2000; Haines III and St-Onge 2012; DeNisi and Murphy 2017; Kalgin, Podolskiy et al. 2018). In this regard, Kuvaas (2011) states that, regardless of the rhetoric about employee PM and its effect on commitment and employee performance, these relationships are mainly assumed rather than empirically tested. The findings of this research show that if employee PM is well designed and implemented, it can benefit the organisation and the public service (De Waal 2007; Verheijen and Dobrolyubova 2007; Kong, Kim et al. 2013).

Consistent with this finding, some prior studies in Vietnam, albeit in the business sector, indicate that employee PM can be well localised in joint-venture enterprises (Thang and Quang 2005; King-Kauanui, Ngoc et al. 2006). Also, some previous studies in the public sector, albeit in other countries, find that employee PM positively impacts job satisfaction (Kalgin, Podolskiy et al. 2018), organisational commitment (Fletcher and Williams 1996), employee motivation (Lee 2017) and organisational performance (Roberts and Reed 1996; Verbeeten 2008; Walker, Damanpour et al. 2010; Gerrish 2016).

The findings from this research increase the confidence of policy-makers and practitioners who are planning to adopt this practice in the public sector in developing contexts. This reinforces the belief that the failure of employee PM may not lie in theoretical problems, but in implementation (Randma-Liiv 2005). The perspectives that view employee PM as "a deadly management disease" are usually based on anecdote rather than rigorous empirical research (Lee 2017). Despite acknowledging persistent problems, high-quality research in both the developed and developing context advocates the use of employee PM because its benefits for employees, organisations and public interest outweigh the costs (Hasnain, Manning et al. 2012; West and Blackman 2015; Lee 2017). Indeed, Schleicher, Baumann et al. (2018) conclude that "PM can be effective across different contexts but the specifics of PM do and should vary across contexts" (p.2226).

Second, along with asserting the applicability of employee PM in developing contexts, this research contributes to the literature by discussing ways to make employee PM more 
effective in developing contexts. Arguably, while there is a general consensus that a good employee PM system can improve organisational effectiveness, there is less clarity about the specific practices that can make an employee PM system effective (Lawler 2003). The present research claims that for a PM system to be effective, its practices should be adopted in concert. The absence of one of these practices may make a system less robust, or even fail.

In reality, this perspective has been mentioned in prior studies, albeit in less systematic ways. For instance, goal-based appraisals will be extremely limited if they are not closely linked to rewards (Shafie 1996; Burns and Zhiren 2010). Meanwhile, rewarding, addressing poor performance and providing feedback may not be successful if there is lack of specific and objective appraisal criteria that pertain to the job (Randma-Liiv 2005; Liu and Dong 2012). A recent study in the U.S public sector indicates that the effectiveness of pay-for-performance will be improved if the employees perceive that the appraisal practice is fairly and objectively implemented (Kim 2016). In a study about introducing PM in the Australian public service, West and Blackman (2015) conclude that employee PM only operates effectively "when these practices work together in a complementary manner to support the achievement of goals and objectives" (p.75).

Third, goal-based appraisal should be a prerequisite when adopting an employee PM system. Goal-based appraisal not only orients individual effort towards organisational goals but also makes other employee PM practices more effective. Pulakos and O'Leary (2011) state that goal-based appraisals curb subjectivity and political motives in the evaluation process. The interviewees in the laggard cluster revealed that their organisations are reluctant to link rewards with performance or address poor performance because they do not trust appraisal results. Consistent with this conclusion, Meng and Wu (2015) argue that the critical reason for the failure of pay-for-performance closely relates to performance appraisal processes. Therefore, some of the most important tasks when adopting an employee PM system are refining organisational objectives, reviewing job descriptions, and then identifying outputs and activities that are important to achieve the organisation's goals. This finding is consistent with previous research that contends that an employee PM system should begin by making organisational strategies and values clear, outlining organisational objectives, and updating job descriptions (Markus and Markus 2004; Pulakos, Mueller-Hanson et al. 2012). In addition, the goal-based appraisal process needs employee participation. The observation from advanced employee PM systems in this study shows that the employees are encouraged to participate in developing organisational goals, job descriptions and performance standards. 
Fourth, in addition to developing objective appraisal criteria, it is necessary to hold raters accountable for appraisal results. This is crucial to curb rating errors derived from political motives, leniency, and personal relationships. A number of studies agree that doing so will make raters pay more attention to information about the ratees' performance and reduce distortion in the appraisal process (Mero, Guidice et al. 2007; Smith 2012). All these measures are to ensure that employees perceive the justice and fairness of the goal-setting and evaluation processes. Once employees are satisfied with their performance standards, they tend to accept the consequences of evaluations.

Fifth, the role of feedback in the employee PM process is virtually undebatable. Risher (2011) argues that employees might not improve their performance if they do not receive honest comments about how well they are performing their duties. However, the way to make the feedback practice fruitful is still in question. In fact, supervisors and co-workers are still reluctant to provide constructive criticism (Posthuma and Campion 2008), particularly in workplaces with face-saving and harmony-keeping cultures. With such cultures, people tend to avoid providing honest and frank feedback (Hofstede 1998; Vallance 1999; Chiang and Birtch 2010).

This research finds that honest feedback may be promoted if the organisation can create a system that allows anonymous feedback. For example, in an effort to embrace 360-degree evaluations, some organisations have developed internet-based appraisal software that can hide the evaluator's identity. This can partially remedy the limitation of traditional collective feedback. Agreeing with this proposition, Latham and Mann (2006) state that anonymous appraisal from peers is more valid than appraisal from of supervisors and subordinates. Besides, the research finds that the negative effect of traditional cultures can be curbed if organisations adopt the performance management of teams and reward employees based on the team's performance. This has proved effective because co-workers are less reluctant to provide honest and frank feedback to poor performers on the ground that these people's performance is negatively affecting the team's performance and well-being. Consistent with this, Jiang (2010) suggests that establishing work-teams and distributing rewards based on the team's performance promote honest feedback from team members.

Sixth, reward-for-performance in public sectors is often problematic and widely disdained because of measurement difficulties, conflicting goals, conflict with public service motivation, union opposition and inadequate government funding (Marshall 1998; Bowman 
2010; Taylor and Beh 2013). There are doubts about the capacity of pay-for-performance to influence employee performance (Perry, Engbers et al. 2009; Weibel, Rost et al. 2009; Taylor and Beh 2013). However, this study finds that reward-for-performance is positively related to the effectiveness of employee PM. The interviewees indicated that a poor connection between performance and rewards is most likely to make technically good PM systems fail because of employees' apathy about the system. This result is consistent with Allan's (1994) study that reported that if appraisal results are merely recorded and placed in personnel files, the system will be viewed as useless and a mere paperwork. As a result, the system will lose whatever credibility it might have had.

Similarly, this research agrees with a study conducted in Portugal that showed that a lack of consequences discredits PM policies because it is "perceived as a source of unfairness and inequity" (Cunha, Vieira et al. 2018, p.683). In a study conducted with Vietnamese SMEs, King-Kauanui, Ngoc et al. (2006) find that incentive pay has a positive and significant effect on the SEMs' performance. In this vein, Risher (2011) argues that linking rewards to performance not only makes employees pay more attention to their performance but also makes managers take more responsibility in the appraisal process because they know that their ratings will have a big impact on the employees' benefit and career. One study conducted in Ghana showed that when salary and other incentives are closely connected to performance, employees have more motivation and expend more effort to increase their productivity (Seniwoliba 2014).

In fact, reward-for-performance might have more leverage in motivating public employees in developing countries where their salaries are usually considerably lower than their counterparts in developed countries and even in the private sector in their countries (Teclemichael Tessema and Soeters 2006; Zientara and Kuczyński 2009; Poor and Plesoianu 2010). In a meta-study of performance-related pay in the public sector, Hasnain, Manning et al. (2012) conclude that incentive programmes in developing countries have more positive results than in western countries and the rationale can stem from the relative magnitude of bonuses compared to basic salaries. While this study finds that effective employee PM uses a broad mix of financial and non-financial incentives, the role of financial rewards for performance cannot be overlooked, particularly in settings such as Vietnam where public sector salaries are not enough to meet basic needs, and are falling behind rising private sector salaries. Money matters, particularly in transitional economies where the attractions of a public sector job might be more about financial security (a stable job) than an intrinsic desire to serve the public. In this regard, 
Shields (2007) concludes that regardless of the inherent difficulties, there is an increasing trend of adopting performance-based pay in both the private and public sector around the world.

While providing empirical evidence to support the introduction of performance-based rewards in the public sector in developing countries, this research acknowledges difficulties when introducing this practice for coping jobs such as administration and policy development jobs because their performance is neither easily measurable nor observable. Hasnain, Manning et al. (2012) also report that performance-related pay tends to be less successful in coping jobs than production jobs (relatively easily measurable and observable) or craft jobs (not observable but relatively easily measurable). Therefore, performance appraisal of these jobs should be incorporated with other criteria such as behaviours and competence. Nankervis and Compton (2006) report that 46 percent of organisations in Australia adopts behaviour-based appraisal as part of their performance management system. Similarly, in the New Performance Appraisal System, the Malaysian government combined work outputs, knowledge and skills, behaviours to evaluate their public employees (Shafie 1996).

Additionally, in the interview process, this research observed that some public organisations in Vietnam pay employees (apart from base salaries) according to so-called "useful time". Accordingly, monthly, employees are required to list works or products they have accomplished. These works are then converted into useful hours that are calculated based on average time to accomplish the work. Consequently, employees' extra income depends on "useful time" rather than actual time they have spent to accomplish those works. While such schemes can be time-consuming at the beginning of implementation and need more research, it can increase the feeling of fairness and improve responsibility. Particularly, they are better than common appraisal practice that mostly relies on traits and abstract criteria such as compliance, honesty and political personality.

Seventh, the findings indicate that organisations using advanced PM not only reward good performers but also seriously address poor performers. The quantitative analysis shows the statistically significant relationship between addressing poor performance and outcomes such as employee motivation, commitment, job satisfaction and organisational performance. This finding may differ from some studies that do not recommend the use of sanctions for poor performers on the ground that sanctions only stop unwanted behaviours in the short term but do not solve the underlying issue (Mitchell and O'Reilly 1983). Additionally, punishments can 
have an adverse impact on work morale and incur union opposition and damages in terms of time and money (Bridges 2013).

However, the results show that the organisations in the advanced cluster both deal with poor performers and have more committed satisfied and motivated staff. The critical point about handling poor performers effectively is having a rigorous appraisal system that includes participation from employees. This result concurs with other studies that support the use of sanctions to improve employee motivation and performance (O'Reilly III and Weitz 1980; Dawson and Billingsley 2000). One quantitative study by Lawler (2003) indicates that the termination of the lowest rated individuals has a strong effect on PM effectiveness in terms of appraisal accuracy, motivation and performance. This is because it signifies that the consequences of the appraisal are very serious, thereby forcing both the supervisors and employees to put more effort into doing it well. Similarly, Dawson and Billingsley (2000) contend that addressing poor performance is an effective solution to make employees accountable for their performance because it sends a strong message that tenured employees will no longer be ensured unconditional lifetime employment. One empirical study in the Malaysian public sector also points out that the introduction of corrective measures makes poor performers more motivated to meet the minimal work requirements (Shafie 1996).

The interviewees in this research suggested that handling poor performers not only enhances the accountability, motivation and work morale of incumbent employees but also improves the quality of staffing by discouraging nepotism and bribery in the recruitment process. This arises because the incentive to being recruited as the result of interpersonal relationships or bribery will be reduced if the candidates perceive that they are likely to be dismissed if they do not satisfy the requirements of the job.

Although addressing poor performers is necessary, this study finds that transferring poor performers to new jobs that better match their competence before proceeding to dismissal procedures is preferred in the public sector. Doing so can reduce grievances and enhance work morale. This is because it signifies that the organisation has the goodwill to retain the employees, but it is concerned with organisational performance and employee performance as well (Osborne and Plastrik 1997; Tong, Straussman et al. 1999). In fact, the respondents in this research reported that some poor performers in their organisations voluntarily left the organisations after being rated and transferred to less demanding positions. This is because they perceived that their institution was no longer a "safe resort" for lazy and incompetent people 
and the new criteria were very objective and specific. Hence, if they did not have the competence and motivation to meet performance standards, they did not have any reason to continue staying in the organisation.

Eighth, although employee participation is not often encouraged in countries characterised by a high power distance culture and paternalism (Aycan, Kanungo et al. 1999; Entrekin and Chung 2001), the results of this research show that organisations will benefit if they can create a friendly and open atmosphere where employees feel comfortable to raise problems regarding their job performance. This finding concurs with previous studies, e.g. Kleingeld, Van Tuijl et al. (2004) and Locke and Latham (2002) that found that employees who participate in setting goals, developing performance standards and feedback have higher commitment and performance than employees who only receive an introduction to employee PM (tell-and-sell) or who are in the control group.

Some research also shows that employee participation positively correlates with increased perception of fairness and the effectiveness of employee PM (Gorman, Meriac et al. 2017). In contrast, a lack of employee participation causes apathy and even resistance because employees perceive employee PM as a tool to control and punish them (Ahenkan, Tenakwah et al. 2018). As a result, a body of research e.g. Gorman, Meriac et al. (2017) and Verbeeten (2008) concludes that employee participation increases the perception of fairness, the sense of ownership and overall PM effectiveness.

For employee participation to be effective, the following prerequisites need to be met. First, the organisation needs to clarify how the PM innovation scheme is important to employees. Employees need to be aware that this process is not only critical for the organisation's survival and development but also benefits them directly (rewards and discipline). Second, employees need to be convinced that the chief executive and other managers are committed, open-minded, impartial and not self-interested in the implementation process. These elements are to ensure that employees can freely voice their concerns without the fear of reprisal. In reality, this might not be as important for developed countries, which have a high level of democracy, but it is particularly meaningful for many developing countries. The reason is that high power distance, paternalism and the process of centralised decisionmaking in these countries make managers reluctant to discuss matters openly with their subordinates (Milliman, Nason et al. 1998; Hofstede 2001; Zientara and Kuczyński 2009). Some interviewees in this research reported that if they realised that their leaders were 
autocratic or half-heartedly democratic, they would refrain from participating in the organisation's affairs.

Finally, the adoption of employee PM not only leads to improved performance but also benefits the public service reforming process in different ways. For example, objective ratings enable recruitment, succession, downsizing and compensation to be more effective. In fact, these reforms are often ineffective because organisations are unable to differentiate between good and poor performers. Employee PM can foster merit principles through evaluating and rewarding employees objectively and fairly, which in turn discourages arbitrary personnel decisions and directs employees to increase their attention to their performance (rather than, say, to seek patronage). In this vein, Hasnain, Manning et al. (2012) contend that employee PM systems, particularly those with performance-related pay, discourage low-ability individuals, while encouraging high ability employees to join public agencies. Thus, employee PM is a useful tool for strengthening a broad range of reforms. These outcomes are crucial for many developing countries where irresponsibility, low work ethic, bureaucracy, poor leadership and corruption are becoming a "bottleneck" for the reform and development process (Drechsler 2000; Ho and Im 2015).

\subsubsection{Findings regarding research question 2}

This research extends our knowledge of contextual factors in the development of employee PM in the public sector. Specifically, it finds that the three contextual factors of agency accountability, HR autonomy and entrepreneurial leadership drive the development of employee PM. Although HR competence by itself does not have a direct effect on employee PM, the interaction between HR competence and entrepreneurial leadership does have a significant effect on employee PM. These findings provide empirical evidence for the claim that the development of employee PM is significantly influenced by contextual factors (Murphy and Cleveland 1995; Haines III and St-Onge 2012). Specifically, the present research sheds light on the effect of contextual factors that are often under-researched in the literature (Carassus, Favoreu et al. 2014). The following section discusses these contextual factors in detail.

\section{Agency accountability}

Consistent with previous studies of public sector reforms e.g. Vian, Brinkerhoff et al. (2012) and Poon, Hung et al. (2009), this study posits that the effectiveness of any reform programme will be modest when institutional and "mechanism" issues are not seriously 
addressed. This research indicates that increased accountability forces public organisations to utilise organisational resources, including HR resources, more effectively. This study is one of the first studies that provide empirical evidence of the effect of agency accountability on the development of employee PM, although the role of accountability in PAR initiatives has been documented. For example, the World Bank commented that in the context of developing countries, "Accountability is an important means for establishing criteria to measure the performance of public officials and for creating oversight mechanisms... without appropriate and effective accountability mechanisms in place, the success of NPM-style reforms would be limited" (Samaratunge, Alam et al. 2008, p.102, 104). Also, this finding confirms the premise of prior qualitative studies that deficient accountability is probably one of the root causes of underdeveloped employee PM in transitional countries (Randma-Liiv 2005; Liu and Dong 2012). In this vein, Ferris, Munyon et al. (2008) also conclude that varying levels of accountability will result in differing levels of PM effectiveness.

An effective way to hold agencies accountable, as suggested by the pilot provinces in this research, is by evaluating chief executives on their organisations' performance. Accordingly, the political future of chief executives (e.g. promotion, reward and demotion) will depend on the extent to which the organisation has achieved goals set by the upper agencies. As a result, this will force chief executives to refine and renovate the use of organisational resources. The literature also documents that the evaluation of chief executives can increase performance accountability, enhance the efficiency of the organisation and mitigate the adverse effects of traditional cultures (Conger, Finegold et al. 1998; Akbar 2011).

In reality, the evaluation of chief executives has already been introduced in some nations around the world such as New Zealand (Norman 2013) and some other OECD member countries (Ketelaar, Manning et al. 2007). In these cases, the chief executives' performance is usually tied to promotion, recognition and financial incentives. However, such practices are often constrained in developing countries. For example, promotion still relies on political personalities and interpersonal relationships rather than competence and work achievement. Collectivism and egalitarianism also impede the provision of different incentives among managers, and between managers and employees. Therefore, reforming accountability needs to go hand in hand with other comprehensive reforms. As a result, there needs to be political commitment from high-ranking leaders in the political system (Samaratunge, Alam et al. 2008)

\section{HR autonomy}


Consistent with some preceding research e.g. Redman, Snape et al. (2000) and Dou, Devos et al. (2016), this research finds that HR autonomy plays a substantial role in the introduction and success of employee PM. In this regard, previous research acknowledges that public agencies operate within an institutional and legal framework that decides their capacity for innovation and change (Birkinshaw, Hamel et al. 2008; Carassus, Favoreu et al. 2014). The delegation of personnel practices encourages managers to look for the mechanisms that maximise benefits while minimising costs in their own circumstances (Hasnain, Manning et al. 2012). Askim, Christensen et al. (2015) conclude that for accountability to be effective, public organisations need autonomy, or operational authority, to best use resources aimed at meeting set targets. Based on a study in the education field in OECD countries, Maslowski, Scheerens et al. (2007) conclude that increased HR autonomy of the schools pertains to teachers' career development, motivation and commitment which in turn improves the students' outcomes. This is understandable because an employee PM system can only operate effectively if it is aligned with the organisation's culture, characteristics and context (Stoskopf 2002; Haines III and StOnge 2012). Indeed, in the present research, advanced employee PM systems tend to be developed in self-financing organisations or pilot agencies that have more autonomy in HR decisions regarding recruitment, appraisal, rewarding, promotion and discipline.

However, in developing contexts, as discussed in Chapter 3, decentralisation is often constrained because higher agencies fear that granting more autonomy can lead to misconduct. They might also deliberately postpone decentralisation to maintain their power and privilege over lower-level organisations. Regardless of this fact, achieving some freedom to innovate is clearly crucial. McCourt and Ramgutty-Wong (2003) contend that as one component of strategic HRM, employee PM needs sufficient autonomy in personnel management. This requires strong determination from political leaders, even at the highest levels of the national assembly in order to promote decentralisation. This corresponds with the conclusion of McCourt and Ramgutty-Wong (2003) that the decentralisation of HR autonomy "requires a substantial commitment of political resources" (p.611).

Although leaders' will is important, the long-term and comprehensive solution is to transform from a process-oriented to result-orient management mechanism e.g. Management by Objectives (MBO) or performance management at the organisational level. Such a mechanism, on the one hand, holds chief executives accountable for the organisation's performance. On the other hand, it allows the organisation to have more autonomy or flexibility to create desirable results. 


\section{Entrepreneurial leadership}

While accountability and autonomy are still limited and need more time to reach a desirable state, chief executives' entrepreneurial leadership emerges as one of the most important factors for innovating employee PM in the present research. Indeed, the innovation of employee PM not only faces difficulties regarding technical issues and resistance within the organisation, but also faces risks from legal systems that are restrictive, ambiguous, or flawed. In the absence of explicit requirements to engage in advanced PM, entrepreneurial leadership plays a crucial role. If the chief executive lacks entrepreneurial spirit and commitment, implementation is highly likely to be postponed.

The results from both the quantitative and qualitative analysis emphasise the notable role of entrepreneurial leaders in the innovation process who overcome institutional and cultural challenges. In fact, compliance with senior agencies has traditionally been highly valued and any innovation without prior permission is not encouraged or even punished (Suutari and Riusala 2001). This conclusion is consolidated by evidence indicating that a number of advanced employee PM systems encounter the risk of degradation or disruption when the incumbent chief executives retire and new leaders lacks the interest and commitment to pursue the innovation process. In this regard, Allan (1994) states that, "Without strong top management backing, the system, no matter how well designed, is doomed to fail" (p.6). Indeed, entrepreneurial leadership is crucial not only in the business sector but also in the public sector.

In addition to entrepreneurial spirit, chief executives must be impartial, not self-interested and willing to sacrifice their own interests for the organisation's benefit. Both employees and line managers only really engage with the PM system when they believe that innovation is merely for the benefit of the organisation. Additionally, chief executives need to be able to persuade and get support from upper-level authorities, e.g. as obtaining a "fence-breaking" mechanism to overcome barriers in the current backward mechanism.

This research is one of the pioneering studies about the role of entrepreneurial leadership in relation to the innovation of employee PM, although the extant literature has reported the relationship between entrepreneurial leadership and other innovative activities in the public sector. For example, a number of studies have found a positive relationship between entrepreneurial leadership and innovation in educational institutions (Bercovitz and Feldman 2004; Yusof and Jain 2009). Accordingly, entrepreneurial leadership is not only the prerequisite for renovating employee PM but also a significant factor for any PAR activity in transitional 
countries where collective leadership, flawed legal systems and process-oriented culture also mean managers avoid taking individual responsibility. Therefore, seeking, nominating and developing chief executives with entrepreneurial leadership and integrity is also needed for the success of the reform process.

\section{HR competence}

Contrary to the initial hypothesis and some previous research that argues that HR competence plays a vital role in HRM innovation (Murphy and Southey 2003), the direct impact of HR competence on the development of PM practices is not supported by the quantitative and qualitative analysis. Instead, HR competence can only leverage its potential in the presence of entrepreneurial leadership. This finding is consistent with the study by Biron, Farndale et al. (2011) that reports that, contrary to the common assumption that employee PM is owned and driven by HR units, chief executives play an active role in its introduction and implementation. In reality, like other transitional and developing countries, HR units are rather reactive and passive in HRM practices in general and employee PM in particular (Taylor 1992; Tong, Straussman et al. 1999; Poor and Plesoianu 2010; Milikić, Janićijević et al. 2012; Berman 2015). Accordingly, instead of proactively proposing strategic HRM initiatives, HR departments or HR units mostly deal with administrative and operational work such as staffing, calculating salaries and social welfare, organising training courses and legalising personnel decisions already made by chief executives (Zhu, Warner et al. 2007; Poor and Plesoianu 2010; Milikić, Janićijević et al. 2012).

Arguably, this issue derives from current institutions in transitional countries. Specifically, these institutions often require personnel practices in lower-level organisations to closely comply with the procedures stipulated by the current regulations and instructions of upper-level agencies. Innovation in HR practices is not encouraged and is likely to lead to risks for the initiators. In such circumstances, HR units may only deliver their full potential if chief executives are proactive and willing to take risks for the renovation. This finding implies that, although HR competence is important, HR units cannot leverage their role without the support and engagement of chief executives.

However, this does not necessarily mean that HR competence is not important. In fact, advanced PM systems usually have competent HR units. Enhancing the competence of HR units is therefore still an important element in continuing to enhance the effectiveness of employee PM. The problem is that HR units need to play a more proactive role in the PAR 
process and organisational change (Taylor 1992; Berman 2015). In order to do this, HR units need to be upgraded with strategic HRM knowledge, insight into the organisation's operations and competence to manage change processes (Ulrich, Brockbank et al. 1995).

Among these, upgrading modern HRM knowledge is perhaps more urgent in the current context. As previously presented, the majority of incumbent HR professionals have very little knowledge of modern HRM except some experience from the centrally planned economy. Meanwhile, employee PM uses principles and theories that differ considerably from those of the centrally planned economy, e.g. competition vs. egalitarianism, explicit accountability vs. collective responsibility, meritocracy vs. seniority and loyalty. It is also noteworthy that although the organisations in the advanced cluster have many reform activities regarding employee PM, there remains a lot of room for improvement. For example, most individual goals are not yet effectively linked to organisational goals. Performance standards mainly focus on outputs rather than outcomes. Some other PM practices such as development training, coaching, and mentoring are rarely adopted although they have proven effective. Therefore, only if HR professionals understand and apply modern HR knowledge effectively, can they gain credibility for participating in strategic decision making, as well as taking strategic roles in the reform process (Stanton and Nankervis 2011).

\subsubsection{Findings regarding research question 3}

These findings reveal that agency accountability, HR autonomy and entrepreneurial leadership not only indirectly, as hypothesised, but also directly affect employee motivation, organisational commitment, job satisfaction and organisational performance.

There are two implications regarding these findings. First, employee PM can be regarded as an effective mediating means through which reform efforts can be channelled into desirable outcomes in terms of improved attitudes and performance. In other words, PAR programmes will be less effective in strengthening the performance of the public service if they do not go hand in hand with a robust employee PM programme.

This reasoning can be demonstrated in the context of Vietnam. In the last two decades, the Vietnamese government has spent a lot of money and effort carrying out PAR activities, but the quality of the public service and the effectiveness of these activities is very poor and far below expectations. One frequently cited cause is that a large number of employees and even managers are not really concerned about their performance and the organisation's performance (CPV 2012; Thu 2018). Many ambitious and costly PAR programmes have failed or were not 
effective because of subjective causes deriving from the implementers' competence and motivation (Vietnam 2016; Thu 2018). In this vein, Ingraham and Kneedler (2000) argue that improving HRM practices should be viewed as a central element in improving the quality of the public service. For post-communist states, the lack of competent and motivated employees is seen as one of the greatest challenges during the transition process (Hesse 1993; Poon, Hung et al. 2009; Cierco 2013).

Second, the analysis result reveals that these contextual factors not only indirectly but also directly affect the outcome variables. These findings show that the improvement of employee performance and public organisations' performance is a complex process that needs comprehensive and systematic reforms. Although the introduction of employee PM is necessary to connect the reform efforts with performance improvement, there are other channels through which accountability, HR autonomy and entrepreneurial leadership can promote employee attitudes and organisational performance. For example, evaluating and ranking organisations can stimulate emulation movements between units. Meanwhile, chief executives with entrepreneurial leadership, integrity and self-sacrifice can win respect from subordinates for being a good role model, which in turn positively affects the employees' work morale and performance. Once again, these findings show that the introduction of employee PM to improve performance needs to be placed in a more complex and broader context. The simplification of this process may be a main reason why many ambitious PM improvement programmes are unsuccessful (Randma-Liiv 2005; Liu and Dong 2012; Ohemeng, Zakari et al. 2015).

\subsubsection{Findings relating research question 4}

These findings reveal that nepotism and personal relationships moderate (weaken) the effect of HR autonomy and entrepreneurial leadership while not moderating the effect of accountability on the development of employee PM.

This research contributes to the literature by exploring the moderating effect of interpersonal relationships and nepotism on the development of employee PM in the public setting in developing countries. Although the moderating effects of interpersonal relationships and nepotism are only statistically significant in some regressions, the research corroborates previous arguments that traditional cultural values in developing countries may hinder or even confound well-designed PM systems (Ohemeng 2009). The analysis results show that nepotism and interpersonal relationships undermine the merit principle that is viewed as the basis of a robust PM system. Indeed, the presence of these factors distorts appraisal results (Bozionelos 
and Wang 2007; Vveinhardt and Petrauskaite 2013), constrains constructive feedback (Ichniowski 1988; Hayajneh, Dwairi et al. 1994), nullifying performance-based rewarding (Hayajenh, Maghrabi et al. 1994; Ouyang 2011). This in turn leads to decreased justice, job dissatisfaction, demotivation and decreased productivity (Hayajneh, Dwairi et al. 1994; Scoppa 2009; Vveinhardt and Petrauskaite 2013).

For these reasons, Cunha, Vieira et al. (2018) suggest that, to improve the quality of employee PM, organisations need to counter well-established cultural values such as nepotism, egalitarianism and personal relationships. Practitioners in developing countries should take into account these factors in the design and implementation of HRM practices (Hofstede 1993; McCourt and Ramgutty-Wong 2003; Bozionelos and Wang 2007; Ohemeng 2009).

One unexpected, but interesting finding in this research is that while these cultural factors weaken the effects of HR autonomy and entrepreneurial leadership, they do not moderate the effect of accountability on employee PM. Holding chief executives accountable for their organisations' performance creates a top-down accountability mechanism. As a result, lowerlevel managers have to attend to the unit's performance. Thus, accountability forces line managers to pay attention to improving employees' and the unit's performance as one of their top priorities. Consequently, the effect of traditional cultural values on decision-making is minimised because if managers continue tolerating these traditional values, their advancement path can be negatively affected.

This finding leads to an important conclusion that accountability plays a crucial role in the reform process in general and employee PM renovation in particular. Indeed, accountability is not only a factor motivating more effective management modes, but is also able to nullify or at least curb the negative effects of traditional cultures on the reform process. Consistent with this finding, Akbar, Pilcher et al. (2012) argue that holding managers accountable by evaluating organisational performance requires changes in operations, managers' commitment and the culture of the organisation. Likewise, Mero, Guidice et al. (2007) conclude that when managers are held accountable for their decisions, they tend to provide more accurate and objective ratings.

One study on implementing performance management in China's provinces indicates that holding managers accountable for their performance closely ties personnel decisions such as promotion, rewarding and demotion to accomplishment indicators (Burns and Zhiren 2010), which can limit the interference of patronage, nepotism and interpersonal relationships. 
Therefore, for developing countries, public managers' accountability is viewed as imperative for not only improving public performance but also controlling corruption and power abuse. Without an appropriate and rigorous accountability mechanism, the effectiveness of reform programmes will be very limited (Porter 2004; Samaratunge, Alam et al. 2008).

As well as asserting the negative effects of nepotism and personal relationships, this research contributes to the literature by making some suggestions to overcome these obstacles. First, organisations in the advanced cluster tend to develop objective appraisal criteria that rely on work rather than personal traits. This is consistent with previous research that suggests that nepotism and personal relationships can be minimised by making personnel practices more transparent and merit-based (Ma, Tang et al. 2015).

Second, it suggests that committed leaders can overcome these problems by holding supervisors accountable for the accuracy and objectivity of their ratings. Consistent with this, Mero, Guidice et al. (2007) and Smith (2012) opine that holding raters accountable for performance appraisals will reduce leniency and personal relationships, which in turn makes rating results more accurate. Similarly, some scholars contend that applying incentives (including punishment) to raters can positively affect the accuracy of appraisal (Harris 1994; Park 2014).

Third, chief executives play an important role in fighting against the effect of traditional cultures. The research reveals that for an employee PM system to be effective, chief executives need to be impartial, enthusiastic and concerned with the benefits for their organisation rather than their own interest. In contrast, if the chief executives recruit relatives and are selfinterested, the innovation of employee PM will not get wholehearted support from employees.

\subsubsection{Findings regarding research question 5}

These findings indicate that communication and training moderate (strengthens) the effect of HR autonomy and entrepreneurial leadership on the development of employee PM. However, none of them moderates the effect of agency accountability.

\section{Communication}

The success of an employee PM system depends not only on how well it is developed but also on the support from users i.e. supervisors and employees (Longenecker and Goff 1992; Kong, Kim et al. 2013; Shahina and Sumod 2015). If users do not have sufficient understanding or belief in its value, the PM system tends to fail (Cunha, Vieira et al. 2018; Ohemeng, Amoako- 
Asiedu et al. 2018). A good communication strategy signifies the organisation's commitment and is key for effective PM implementation (Cunha, Vieira et al. 2018).

This study provides empirical evidence in a developing context to advocate previous studies (mostly conducted in developed countries) that suggest communication increases the likelihood of success of employee PM adoption (Kotter and Schlesinger 1979; Armenakis and Bedeian 1999; Biron, Farndale et al. 2011; Kinicki, Jacobson et al. 2013). Specifically, it indicates that the adoption of organisational communication make two of the three antecedents (i.e. HR autonomy and entrepreneurial leadership) more influential in the success of employee PM. Accordingly, communication mitigates against the fear of unexpected consequences and the bureaucratic burden perceived from adopting employee PM (Nadler 1997; Bordia, Hobman et al. 2004; Kong, Kim et al. 2013). Additionally, communication helps overcome obstacles of traditional cultures. It has been said that in transitional countries such as Vietnam, cultural values of process-orientation, egalitarianism, collectivism, fatalism and uncertainty avoidance are still very pervasive. Unfortunately, these values often contradict the values of an authentic employee PM system such as emphasising a result-oriented culture and increasing individual accountability. Therefore, communication not only helps employees to reduce uncertainty and increase their understanding of the logic and need for change, but also helps them gradually accept the values of employee PM (Bordia, Hobman et al. 2004; Kong, Kim et al. 2013). In a study of the Ghanaian public sector, Ohemeng, Amoako Asiedu et al. (2018) found that communication plays an important role in institutionalising employee PM in the public sector.

This study also points to suggestions about how communication can be effectively introduced. First, rather than delivering a single speech at the beginning of the implementation process, communication should be continuous. The organisations in the advanced cluster report that they organised several meetings, as well as using other events to raise awareness of the importance of employee PM innovation. Senior managers also need to be very persistent to gain support and acceptance from supervisors and employees. In a study in South Africa, Mafini, Pooe et al. (2014) suggest that employees need to clearly understand the reasons behind the introduction of the innovation, otherwise it is doomed to failure.

Second, the communication process aims to engage stakeholders in the innovation by clarifying how it will further their interests. For example, the innovation of PM enables employees to improve their income and well-being. Meanwhile, for managers, employee PM makes managerial activities more effective rather than imposing an administrative burden. In 
this regard, Armenakis and Harris (2002) emphasise that communication needs to help employees perceive potential benefits from the proposed change.

Third, for communication to be effective, it should be conducted in democratic ways. Although democratic communication may not be a problem for western countries, it is important for many transitional countries where employees' voices are seldom considered due to managers' top-down and undemocratic management style (Maczyński, Jago et al. 1994; Zientara and Kuczyński 2009). This research recommends that for communication to be effective, it needs to be authentically democratic. Democratic two-way communication engenders a feeling of ownership in employees, and increases their commitment and acceptance, even of unfavourable decisions (Greenberg 1987; Cornell and Herman 1989; Chawla and Kevin Kelloway 2004)

\section{Training}

As with communication, the analysis result proves that training for supervisors and employees in utilising employee PM systems can promote the development and effectiveness of employee PM. This finding converges with a number of previous studies that report that training has a positive impact on the development and outcomes of PM. For example, Cavalluzzo and Ittner (2004) and Haines III and St-Onge (2012) find a significant association between training and the development of employee PM, such as developing objective criteria, providing constructive feedback, coaching and rewarding. Meanwhile, a study by Tung, Baird et al. (2011) reports that training in PM is significantly related to the development of employee PM and the effectiveness of outcomes such as motivation and commitment.

In this vein, Risher (2011) explains that the critical factor for an effective employee PM system does not lie with the HR professionals but with immediate supervisors. Hence, supervisors need skills to supervise and manage their staff. Once trained, supervisors tend to support the system and provide more accurate ratings (Gorman, Meriac et al. 2017). Accordingly, Longenecker and Goff (1992) state that "appraisals without training are a sure route to ineffectiveness, frustration, and dissatisfaction" (p.22). Therefore, some research proposes that training should be included in the adoption of employee PM, particularly in developing contexts where the competence of managers is usually limited (Randma-Liiv 2005; Liu and Dong 2012; Bawole, Hossain et al. 2013).

Apart from asserting the role of training in the development of employee PM, the research provides some suggestions on how to make training effective. First, training needs to 
help users, both employees and supervisors, to be familiar with the new employee PM systems. This is particularly necessary for complex systems such as those using online appraisal software. Davis (2015) claims that supervisors will be very reluctant to provide honest feedback if they do not have the skills to monitor and communicate feedback effectively. With regard to this opinion, Emerson (2002) and Hansson, Backlund et al. (2003) contend that training needs to help supervisors and employees better understand relevant concepts and principles as well as acquiring new skills to participate in new systems effectively.

Second, there needs to be multiple training courses to ensure that the users understand and implement employee PM practices sufficiently and correctly. Daley (1992) suggests that organisations should periodically provide training programmes with the length of one to two days for users.

Third, training courses should enable employees to raise their voices, thereby reinforcing transparency and acceptance. In this regard, Lawler, Benson et al. (2012) posit that training enables employees to understand their roles and rights in the implementation process in terms of fairness and accountability. Finally, training needs to give supervisors more understanding of their roles and responsibilities in the implementation process, thereby minimising appraisal errors such as biases and leniency.

\subsubsection{Other findings from the qualitative data}

Apart from the finding related to the initial hypotheses, the qualitative analysis revealed some other important findings. The following section will discuss these findings.

First, despite not being the initial target object, this research indicated that corruption could undermine employee PM in different ways such as distracting chief executives from innovating employee PM as well as undermining merit principles. Also, it revealed that when the chief executives were corrupt and only concerned about their interest groups, employee PM, particularly pay-for-performance, could accelerate conflict within the organisation rather than enhancing employee motivation and performance. To date, the impact of corruption on HR practices and employees' perception has been discussed in many studies ( $\mathrm{Gu} 2013$ ). However, research investigating the effect of corruption on the implementation of employee PM is still sparse. This finding suggests that the introduction of employee PM is more challenging in the public sector where corruption is prevalent. Therefore, anti-corruption activities will be an important antecedent to promote the development of employee PM. 
Second, in addition to the contextual variables and corruption, this research explored that job types could affect the development of employee PM. Specifically, it found that employee PM systems of universities and hospitals (public service delivery organisations) were more developed than administration agencies. This finding agrees with previous studies, e.g. Hasnain, Manning et al. (2012), suggesting that the employee PM systems involving production and craft jobs (observable and measurable jobs) tend to be more effective than the employee PM systems regarding coping jobs (not easily measurable and not observable). This adds more voice to confirm that job types should be paid more attention when introducing employee PM.

Finally, although the findings indicate that employee PM is an effective tool to improve employee attitudes, organisational performance and other reform efforts, it can lead to unwanted consequences. Bevan and Hood (2006) argue that the introduction of PM can lead to the distortions of measures and gaming. The research observed some side effects as well, particularly regarding pay incentives. As presented in the preceding chapter, seeking income resources to pay-for-performance can lead to the conflict of benefits between public organisations and the public interest, particularly for the services that lack competition from the private sector and close monitoring from upper-level agencies. As others have noted, policymakers and executives need to be 'on the lookout' for gaming and disincentives in such systems. Also, it is important to enhance citizen monitoring of the operation of public organisations.

\subsubsection{Proposing a theoretical model from the findings}

Based on the initial conceptual framework and the new findings from data analysis, a theoretical model of the implementation of employee PM in the public sector in developing countries is proposed (see Figure 6-1 as below). Accordingly, in addition to the initially hypothesised variables, the model included some new variables such as anti-corruption, job types and unwanted outcomes as specifically discussed below.

For contextual variables, apart from accountability, HR autonomy, entrepreneurial leadership and HR competence, the research supplemented anti-corruption and jobs types into the list of contextual variables. As discussed, chief executives with anti-corruption attitude enable the implementation of employee PM. Meanwhile, service delivery organisations tend to have more advanced PM systems compared to administration organisations because job performance are easier to measure. It is worth noting that HR competence only makes a difference when the leaders are entrepreneurial.

With regard to the employee PM practice, although these five employee PM practices are important components of an effective PM system, there was some adjustments to make them 
more suitable to developing countries, particularly in the cultures where face-saving and harmony-keeping are high. Accordingly, feedback will be more effective if it is conducted in anonymous ways. Meanwhile, rewarding should be relied on both individual and collective performance. These aim to encourage co-workers to provide more honest and frank feedback to each other. For addressing poor performers in the public sector, it is advisable that organisations should transfer these people to less demanding jobs before applying stricter measures if they continue to fail to fulfil the new jobs.

For cultural factors, apart from nepotism and personal relationships, the research also found that egalitarianism made an employee PM system less effective because it discouraged pay-forperformance and merit principles. Also, it impeded setting challenging goals and appraising objectively.

With regard to intervention practices, aside from communication and training, evidence from the qualitative data demonstrated that holding raters for appraisal results can minimise the negative effect of political motives, leniency and personal relationships, thus promoting the effectiveness of employee PM.

Finally, in addition to employee motivation, organisational commitment, job satisfaction and organisational performance, the introduction of employee PM can engender other desirable outcomes for public organisations and the public interest. As discussed in section 6.2, addressing poor performers is likely to discourage nepotism and bribery in the recruitment process, thus discouraging low-ability individuals. Meanwhile, pay-for-performance can encourage high ability employees to join public organisations. Therefore, it can lead to the improvement of recruitment and selection. Additionally, goal-based appraisal enables to differentiate between good and poor performers more objectively and fairly, thereby making succession planning and staff downsizing more effective. However, the introduction of employee PM is likely to cause unwanted outcomes. Poor design, coupled with the presence of corruption, can mean that the implementation of employee PM can accelerate conflict between employees. Meanwhile, with a lack of rigorous policies and sound monitoring mechanism, PM implementation can compromise the public interest when the public organisations seek ways to maximise their benefit.

In addition to the variables discussed above, one box of institutional setting was supplemented into the model. Accordingly, a wide range of variables related to institution, culture and capacity explored through reviewing the literature and analysing data was added. This gives readers have a better insight into the institutional context where employee PM is 
carried out, thus enabling them to have a better understanding of the rationales, difficulties and likely outcomes of the implementation of PM. 
Figure 6-1: Conceptual framework of the implementation of

\section{Institutional Setting}

performance management in the public sector

- Weak accountability, ambiguous missions

- Weak capacity and weak monitoring

- Collectivism and high uncertainty avoidance

- Confucianism and face-saving culture

- High power distance and top-down management

- High centralisation in personnel management

- High corruption

- Rigid employee protection

- Low salary and poor motivation

- Low performance of public employees

- Ineffectiveness of many PAR programmes

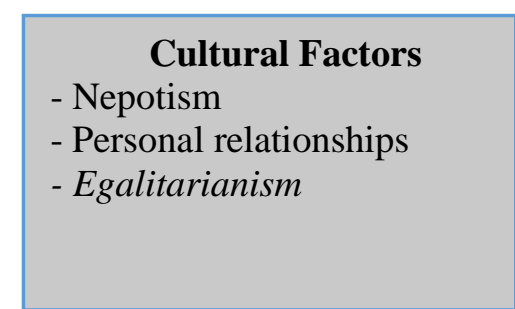

Contextual Variables
- Accountability
- HR autonomy
- Entrepreneurial leadership
- HR competence
- Anti-corruption
- Job types (service delivery organisations (+)
Administration organisations (-)

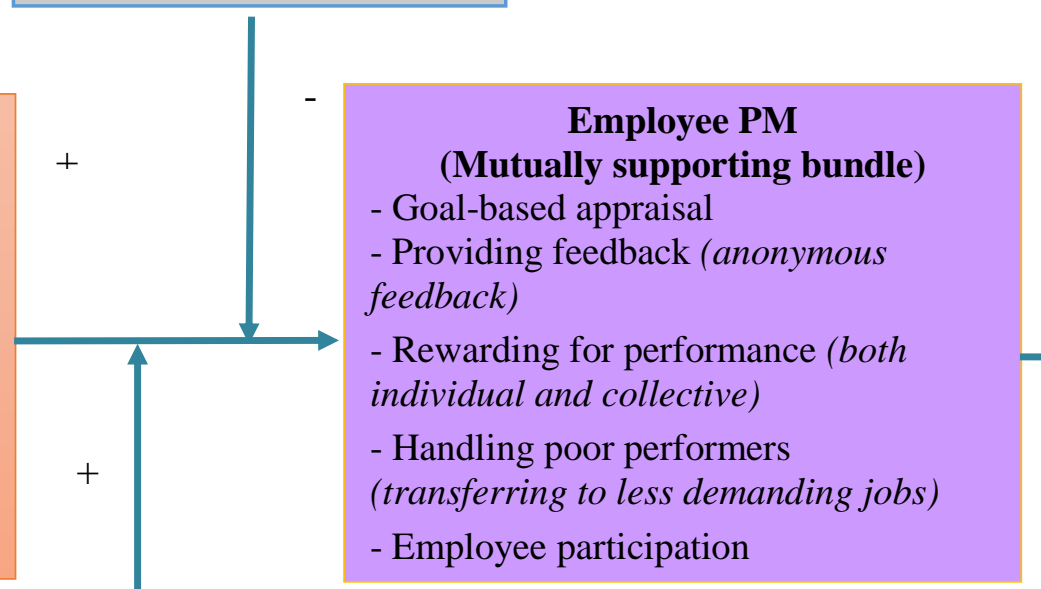
Desirable Outcomes
- Employee motivation
- Organisational commitment
- Employee satisfaction
- Organisational performance
- Improved recruitment and selection
- Succession and downsizing
Unwanted outcomes
- Conflict between employees
- Conflict with the public interest

\section{Intervention practices \\ - Communication \\ - Training \\ - Holding raters accountable \\ for appraisal results}

194

Note: The new variables added to the initial conceptual framework are shown in italics 


\subsection{Contributions}

The need to improve employee performance and PM systems in the public sector in developing countries has been highlighted by many researchers (Tong, Straussman et al. 1999; De Waal 2007; Poon, Hung et al. 2009; Berman 2015; Ohemeng, Zakari et al. 2015). This research makes some theoretical and empirical contributions to this area.

\subsubsection{Theoretical contributions}

An important theoretical contribution of this research is proposing a formula for the effective implementation of PM in developing countries. Accordingly, the five PM practices should be deployed in concert rather than have individual practices deployed in isolation. Prior studies have reported that the failure of PM systems can derive from a lack of integrated PM practices. For example, appraisals will be ritualistic if not being connected to rewards or discipline (De Waal 2007; Liu and Dong 2012). Pay-for-performance and addressing poor performers will be less effective if appraisals are not based on objective appraisal criteria (Mwita 2003; Randma-Liiv 2005). Meanwhile, goal setting and feedback may not be helpful if there is no participation and input from employees (Chiang and Birtch 2010). Hence, this research claims that for employee PM to be effective, the five PM practices, including goalbased appraisals, feedback, employee participation, rewarding and handling poor performance need to be integrated because they can reinforce each other. This finding responds to recent calls for investigating how employee PM practices interact with each other and how these PM practices work together to induce performance improvement (Schleicher, Baumann et al. 2018).

Second, this pioneering research explored and tested the effect of contextual variables in implementing PM in the public sector. As a result, it found the significant influence of accountability, HR autonomy and entrepreneurial leadership on the development of PM. Although these have been discussed in various ways, they have seldom been applied to this area or examined in combination (Taylor 1992; Burns and Zhiren 2010; Berman, Chen et al. 2017). This research contributes to the literature by showing that contextual factors are more influential than what they have previously been thought to be. Although HR competence does not have a direct effect, it can accelerate the effect of entrepreneurial leadership in innovating employee PM. Additionally, despite not being examined by quantitative data, qualitative data provided convincing evidence about the effect of anti-corruption and job types on the development of employee PM.

Third, this research expands the current understanding of the role of employee PM. Specifically, it explored that PM is not only a tool to improve organisational performance, but 
also a mediator to transmit other PAR initiatives. Like many other developing countries, Vietnam introduced several reform activities such as decentralisation, restructuring and capacity building. However, desirable outcomes were far from expectations. Therefore, there is something missing and employee PM can be an agent that can transmit the effect of reform activities on desirable outcomes. This research contributes to the literature by pointing out that employee PM can function as a mediator between reform efforts and desirable outcomes. This finding strengthens the belief that PAR efforts will be less effective without sound employee PM systems.

Fourth, this research extends our understanding of the effect of traditional culture on employee PM. Specifically, it indicates that interpersonal relationships and nepotism significantly impeded the development of employee PM. To date, the effect of culture on performance appraisals and performance management has been widely discussed in the literature (Berrell, Wright et al. 1999; Aycan 2005; Ohemeng 2009). However, the majority of those studies are theoretical. Although some empirical research has been done, most of them use national cultural dimensions proposed by Hofstede such as power distance, uncertainty avoidance, individualism and masculinity (Hofstede 1984) and more strongly reflects the western contexts ( $\mathrm{Gu} 2013)$. This research is one of the very few studies that investigate the effect of nepotism and interpersonal relationships on the development of employee PM. This gives scholars a greater understanding of the effect of traditional culture on the introduction of employee PM. Particularly, it partially shed light on why the introduction of PM in developing countries is more challenging than that in their developed counterparts.

Fifth, although there is no shortage of research on the role of communication and training in organisational change e.g. Elving (2005) and Kotter and Schlesinger (1979), studies of the actual impact of communication and training on the introduction of employee PM are limited and usually theory-based. This research contributes to the literature by providing empirical evidence to support the adoption of these intervention practices in the implementation of employee PM in the public sector in developing countries.

Finally, the research offers a theoretical model (described in Figure 6-1) to illustrate a relatively comprehensive picture about PM implementation. Specifically, it describes relationships between antecedents and employee PM as well as between employee PM and likely outcomes (either desirable or unwanted). Additionally, a wide range of institutional variables found as influential factors was included in the model. Therefore, this model is useful for future researchers to utilise or test in other developing contexts. 


\subsubsection{Empirical contributions}

First, the research provides empirical evidence in the contemporary debate about the applicability of employee PM in the developing context. This is significant because the rate of failed PM schemes is relatively high and there is a belief that employee PM is not compatible with developing countries due to problems concerning institutions, capacity and culture (Mendonca and Kanungo 1996; Vallance 1999; De Waal 2007; Ho and Im 2015). The findings provide convincing evidence that, if well-designed and implemented, employee PM is most likely to induce desirable outcomes such as increased employee attitudes and organisational performance. Hence, the failure of reported PM projects may derive from problems of implementation rather than theoretical defects.

Second, the general consensus of past studies is that pay-for-performance schemes are not very effective in the public sector, and can be very harmful (Perry, Engbers et al. 2009). However, consistent with recent research, e.g. Hasnain, Manning et al. (2012) and Risher (2011), this research asserts that pay-for-performance is an important component in the employee PM system, at least in Vietnam and many other developing countries. The respondents disclosed that not connecting performance with rewards and pays makes employees apathetic about the system and their performance. The role of financial rewards for performance cannot be overlooked, particularly in settings such as Vietnam where public sector salaries are not enough to meet basic needs and are falling behind rising private sector salaries. For many developing and underdeveloped countries, the attractions of a public sector job might be more about security (a stable job) than an intrinsic desire to serve the public.

Finally, to date, the research on HRM practices in general and employee PM in particular in the Vietnamese public sector is relatively scarce. Such studies are scarce in the public sector in transitional countries as well (Zupan and Kaše 2005; De Waal 2007; Jankulović and Škorić 2013). The literature review could only find a few studies of employee PM conducted in the Vietnamese context e.g., Kamoche (2001), Thang and Quang (2005) and Stanton and Pham (2014). However, these studies were largely conducted in the private sector. Although some research was conducted in the public sector, it mostly targets administration reforms e.g. Painter (2003) and Acuña-Alfaro (2009), civil service reforms e.g. Poon, Hung et al. (2009) and corruption e.g. Vian, Brinkerhoff et al. (2012), and Gregory (2016). Studies of employee PM in the public sector are very limited and mostly focus on the appraisal practice e.g. Phan (2014). 
To the best of the author's knowledge, this is one of the pioneering studies of introducing employee PM in the Vietnamese public sector. This work contains important background information about the history, culture and current issues facing Vietnam's administration, which should be useful for scholars who are interested in the public sector of Vietnam and transitional countries.

\subsection{Implications}

Based on the findings, this research has a number of implications for policy-makers, managers and researchers.

\subsubsection{Implications for policy-makers}

This research finds that the strategic solution for promoting advanced employee PM is setting the right institutional context beforehand. The following section presents six strategic solutions that should be embraced to promote employee PM in the public sector.

The first strategic solution is holding chief executives accountable for the organisation's performance. Doing so aims to make the chief executives focus on improving organisational performance rather than seeking patronage. As a result, chief executives will pay more attention to effectively managing human resources aimed at fulfilling the organisation's goals assigned by the upper-level agencies. In this vein, Haines III and St-Onge (2012) declare that, "When human capital is valued, the PM system can deliver its full potential" (p.1170). Increasing accountability also constrains nepotism and personal relationships that negatively affect the development of employee PM. This is because if chief executives continue to tolerate nepotism and prioritise personal relationships in recruitment and other HR practices, they will jeopardise their political careers as their organisations will continue to perform poorly.

An effective solution to hold chief executives accountable is using the organisations' performance as a core criterion to evaluate them. This can be done by adopting Management by Objective (MBO) or performance management at the organisational level. Accordingly, the upper agencies must clarify requirements and goals that are connected to the organisation's function and mission in each period (Boice and Kleiner 1997). It will be necessary to articulate the rewards that chief executives will receive if their organisations fulfil their goals e.g. salary increase or promotion. On the other hand, they are likely to be demoted if their organisations fail to fulfil the agreed goals. 
The second strategic solution is delegating more authority, including HR authority, to public organisations. Holding chief executives to account will be not effective or feasible if they have insufficient resources and lack the autonomy to use resources in the most optimal way. Accordingly, public organisations should have more authority in staffing, career development, pay-for-performance, promotion and discipline. Arguably, if chief executives do not have the ability to reward good performers and deal effectively with poor performers, the system will become ritualistic and ineffective. However, decentralisation and autonomy need to be accompanied by increased accountability, monitoring and inspection aimed at preventing the abuse of power, gaming, favouritism, nepotism and corruption. This aims to avoid manipulating employee PM for personal interest as well as preventing the conflict of benefits between pubic organisations and the public interest.

The third strategic solution is seeking, nominating and developing chief executives with entrepreneurial leadership capabilities. Public organisations need to proactively reform to capture opportunities and deal effectively with new challenges. Hence, the reform process needs people who are keenly interested in and capable of pursuing innovative programmes. Like other reform activities, renovating employee PM needs chief executives who have the vision, capability, innovative spirit and especially courage to take risks (Berman, Chen et al. 2017). Consistent with this reasoning, the findings in the research indicate that in the transition period, entrepreneurial leadership had become a salient factor driving the development of employee PM. Therefore, upper-level agencies and policy-makers should pay attention to developing and fostering entrepreneurial leadership. Accordingly, selection criteria should underscore leadership competency, work achievements and entrepreneurial spirit rather than political personalities, loyalty and compliance. Simultaneously, the procedure for appointing chief executives should be carried out in open and transparent ways. Doing so not only enables to select the best person among candidates but also encourages candidates who are courageous and willing to take risks by participating in competitive examinations.

The fourth strategic solution is strengthening the capabilities of HR professionals in public organisations. As stated above, decentralising HR management authority is necessary to ensure that public organisations have the flexibility needed to reform employee PM. However, decentralisation might be ineffective or even counterproductive if the subordinate organisations have insufficient capability to assume the delegated tasks. Additionally, employee PM is seen as a relatively sophisticated assignment. It is not easy to duplicate an outside system because organisations have different operational functions, financial resources and organisational 
culture. Hence, HR units need to have sufficient knowledge of HRM, change management and the government's regulations to develop employee PM systems that suit the organisation's circumstances and current regulations.

Accordingly, the central agencies in charge of managing public employees such as the Ministry of Home Affairs or State Services Commission should deliver training courses on HRM from a basic to advanced level for HR professionals working in public organisations. It would also be useful to organise workshops on employee PM so that they can share successes and failures during the implementation process. Additionally, it is necessary to equip chief executives with HRM knowledge. This is because they will only support HRM initiatives wholeheartedly and effectively if they really understand it.

The fifth strategic solution is amending the current HR management regulations. Like many other transitional and developing economies e.g. China (Liu and Dong 2012), Estonia (Randma-Liiv 2005), and Ghana (Ohemeng, Zakari et al. 2015), HRM policies in the Vietnamese public sector are obsolete and influenced by Soviet-style management practices. For example, promotion still rests on political quality, pay depends on seniority and egalitarianism, and employment relies on the rigid career-based system. Therefore, besides decentralising autonomy to subordinate organisations, some current HRM regulations need to be amended to align with modern HRM trends that support the implementation of employee PM. The following are some examples. The first is revising appraisal criteria so that they adhere to job performance rather than personal traits and abstract quality. The second is allowing public organisations to have a fund for pay-for-performance alongside the base salary. The third is transforming the career-based system into a position-based system. This aims to ensure that public employees will be recruited, placed, appraised and paid according to their job positions and performance. This is important because the current career-based system means many job positions in public organisations are not filled by the right persons. On this issue, the respondents reported that the difference between a job specification and the incumbent's competence makes goal setting and rating difficult and ineffective.

The sixth strategic solution is to develop guidelines for embracing employee PM. This is because employee PM is a relatively new and sophisticated practice for many developing and transitional countries. Such guidelines can reduce confusion and increase the confidence of subordinate organisations in the implementation process. The main contents of the guidelines should include: (i) developing KPIs for the organisational level and performance standards for 
the individual level; (ii) conducting an appraisal procedure; (iii) providing feedback; (iv) connecting rewards such as pay and promotion to the performance of individuals and teams; (v) dealing with poor performers; and (vi) promoting employee participation.

\subsubsection{Implications for practitioners}

The following are some implications for practitioners.

First, employee PM is best treated as a bundle of integrated practices, rather than sampling a few. It is critical to ensure that employees are appraised by the criteria relating to their jobs and the goals set at the beginning of the performance period. In addition, good performers need to be rewarded with financial awards as well as promotion opportunities while continuously poor performers need to be transferred to less demanding jobs before proceeding to termination if their performance remains unsatisfactory.

Second, performance-based pay should be determined on the basis of both individual performance and team performance. This not only aims to encourage cooperation between members of the team but also promotes honest feedback. The findings show that once employees' bonuses depend on the team's performance, they tend to provide frank comments to poor performers or lazy co-workers. As a result, to avoid receiving unwanted comments, the poor performers have to adjust behaviours, increase effort and upgrade skills. Additionally, to get employees involved the employee PM process, the size of incentive needs to be meaningful. Rules of thumb can be dangerous, but the message here is that money counts, and rewards need to be meaningful to raise performance. Group incentives, as found in this study, might be particularly effective in collective societies. Finally, non-financial rewards such as study leave, job rotation and particularly career development opportunities are shown to be effective in motivating employees.

Third, this research proposes that addressing poor performers should be incorporated into an employee PM system, at least in the public sector in developing countries. While goal setting, evaluation, coaching and performance-based rewards are widely discussed in the employee PM literature, addressing poor performance tends to be neglected in employee PM schemes. There are some perspectives that the use of sanctions for poor performance is not advisable due to its adverse consequences on work morale and the likely damage derived from grievances and litigations. The present research proves that handling poor performers is an important component in differentiating effective PM systems from others. In fact, the problem of poor performers is usually more serious in developing countries than in developed countries. For 
many developing countries, rigid career systems, coupled with traditional values such as nepotism and interpersonal relationships, has led to a prevailing perception that having a job in the public sector is a "safe haven" for lacklustre performers. The inclusion of this practice will make employee PM systems more effective in addressing chronic issues in the public sector such as inertia, irresponsibility and nepotism.

Fourth, supervisors need to be held accountable for their rating. Given time-consuming rating processes, political motives and cultural factors such as face-saving, harmony and personal relationships, supervisors might not assess actual performance objectively and fairly. Gorman, Meriac et al. (2017) conclude that the lack of rater accountability is one of the primary reasons disrupting the whole employee PM system. To correct such behaviours, organisations should hold supervisors accountable for their rating by stipulating that providing objective and fair appraisals is one of their most important tasks. Failure to provide objective ratings can be viewed as not fulfilling managerial job requirements and lead to adverse consequences.

Fifth, to get the support of employees for the innovation, organisations need to develop good communication strategies. It is important to make employees understand the importance of the innovation to the organisation's survival, as well as their own interests. Communication mitigates the fear of the change by making the procedures transparent to all employees. Moreover, for communication to be effective, it should be a democratic and two-way process to allow employees to voice their concerns. Once employees understand that the innovation process is transparent and for the benefit of the organisation, including themselves, they are more likely to support and accept it.

Sixth, training should be provided for supervisors and employees before and during the implementation process. Knowledge and skills needed by supervisors include setting goals, supervising performance, providing feedback, discussing developmental needs and solving conflicts. Meanwhile, training for employees should aim to increase their awareness of the purpose and procedure of the new PM system as well as gaining familiarity with it.

Finally, to have a robust employee PM system, organisations need good preparation and investment in terms of time, effort and financial resources. The determination and persistence of chief executives is critical as well. Normally, employee PM systems have to experience several revisions, even pilot schemes before reaching an acceptable level. This process can take three to five years or even longer. The observations from organisations with advanced PM systems show that chief executives often regarded it as one of the top priorities in their terms. 
They themselves spend a lot of time observing, reflecting and continuously adjusting the system to make it better and better.

\subsubsection{Implications for researchers}

Apart from suggestions for policy-makers and practitioners, this study has some suggestions for researchers.

First, in interviews, note taking should be always considered as an alternative to digital recordings, particularly for sensitive topics and those conducted in Asian countries. The experience of conducting interviews in this research revealed that, although using digital recording is usually suggested and very popular in interviews (Bryman 2015), it can inhibit interviewees from speaking out candidly and freely about what they think because of fear of unexpected consequences. This is particularly true in the context of Vietnam and China where people do not want to be held responsible for the information they speak out about, particularly when this information can displease someone in authority (Gu 2013; Phan 2014). Therefore, to encourage informants to provide honest and valuable information regarding sensitive topics in the public sector, the researcher should consider using note taking rather than asking to use digital recordings.

Second, establishing a rapport with interviewees, particularly for research in the public sector setting, is very important to acquire valuable information. It has been said that accessing potential public organisations to collect data is often difficult for researchers, particularly in a society where information available to the public is limited. In such situations, direct contact with potential interviewees without an introduction by credible sources can result in refusals or the acquisition of less valuable information. Therefore, researchers should seek prestigious people e.g. leaders in the upper agencies or people having close personal relationships with senior managers, to provide an introduction in advance. Having accessed the targeted people in the organisation, it is important to articulate the value of the research, particularly how it relates to the current interests of the organisation. In the present research, the author articulated that the objective of the research is to help policy-makers revise current policies, while enabling practitioners to have a better understanding of employee PM. In fact, after outlining existing issues regarding employee PM in the public sector and potential outcomes of the research, the researcher received the interest and support of many chief executives. As a result, the process of data collection became easier. 
Third, giving a small gift to interviewees is advisable, particularly in a society that appreciates social relationships. Accordingly, besides introducing the researcher's identity and the measures to keep the information confidential, it is also a good idea to present a small gift before the interview begins. The value of the gifts does not need to be high, but it is a custom in many Asian countries to manifest the researcher's appreciation with regard to the information shared by the informants. In this regard, Robertson and Hale (2011) argue that offering a gift (koha) helps to develop a friendly rapport with interviewees. Even though the value of the gift is unlikely to be high, this strategy enables interviewees to see the interviewer as a visitor rather than a formalised interviewer, making them want to reciprocate by telling their stories.

\subsection{Limitations of the thesis}

Like almost all other studies, this study has some limitations.

First, employee PM is a relatively broad concept with a variety of practices (Schleicher, Baumann et al. 2018). However, this study could only investigate the main employee PM practices that have already been adopted by public organisations in Vietnam. Although other employee practices such as coaching and development training are considered important components of employee PM (Bilgin 2007; Latham, Borgogni et al. 2008; Haines III and StOnge 2012), they were not included in the research. The reason is that the preliminary interviews revealed that these practices were virtually unused by public organisations in Vietnam. Therefore, although items for these constructs had been developed beforehand, they were then removed from the final questionnaire.

Second, no study can examine all factors. While this study examined some important contextual factors, several other factors such as organisational size, job type, technology, corruption and political environment remain unexplored. For example, one factor that is very influential but not sufficiently investigated in this study is the nature of jobs, e.g. jobs in service delivery organisations versus jobs in policy development agencies. Although the research found that employee PM in policy development agencies tends to be less advanced than service delivery organisations, it did not provide in-depth explanations for this, or suggestions for improvement. Additionally, while corruption was found to be a factor impeding the development of employee PM in the public sector, it was not thoroughly investigated. Further research might also be needed to examine the effect of these factors on the development of employee PM. 
Third, the sample for the quantitative analysis was purposively drawn from the population. Therefore, the findings may not sufficiently represent the target population. However, the main purpose of this study is not to make generalizations about the use of employee PM but to explore the factors driving the development of employee PM in Vietnam. Because the organisations that have adopted advanced employee PM are still limited, purposive sampling was needed to ensure an adequate range of employee PM systems with different development levels. Additionally, to increase the representativeness of the sample, an effort was made to select organisations that can represent radical characteristics of public organisations such as central versus local agencies, administration versus service delivery institutions, and large-scale versus small-scale institutions.

Fourth, in this research, all the independent and dependent variables were collected at the same time rather than through longitudinal data. Therefore, some results such as bivariate correlations can only indicate the correlations between variables rather than causal relationships. However, multinomial logistic regression that was used in this research has been used in many other studies to investigate causal relationships between predictor variables and outcome variables (Horgan and Muhlau 2003; Yi 2013). Additionally, the qualitative data provides some validation of the proposed causal direction from the contextual factors, to employee PM practices, to better job attitudes. These can consolidate the validity of the conclusion about the predictive role of the antecedents on the development of employee PM. Further studies might seek evidence of longitudinal effects in future years or perhaps use experimental designs.

Fifth, the measures are necessarily based on perceptions as objective data are unavailable on matters of, say, job attitudes and motivation (Podsakoff and Organ 1986). Other sources of objective data such as the organisational performance of public organisations are also unavailable in Vietnam. This is likely to lead to personal bias deriving from social desirability and other causes (Fisher 1993; Yang and Pandey 2009). However, Dess and Robinson (1984) and McCracken, Mcllwain et al. (2001) assert that when objective data is unavailable, subjective data should be used. This is reasonable because employee perception is strongly related to objective measures (Boyd, Dess et al. 1993; Yang and Pandey 2009). In a study of 42.934 correlations published in 581 articles, Crampton and Wagner III (1994) conclude that although self-reporting methods can produce concept-concept inflations, this effect is not as broad and comprehensive as critics' envision. Furthermore, in the present research, this issue was minimised by clearly stating to the participants that all information would be strictly 
confidential and safely managed, as well as emphasising that their honest responses were very important for the research. In fact, the consistency of results across the clusters of organisations and triangulation with qualitative data analysis provide validity for the conclusions drawn.

Sixth, employee performance, as one desired outcome of employee PM, was not included in the research. The reason was that self-evaluation of an employee's performance is likely to be biased. This issue can normally be solved by using a dyadic survey with supervisors' evaluations. However, this exceeded the researcher's capacity in terms of time and resources. Therefore, the research used work motivation, organisational commitment and job satisfaction as a replacement. Nevertheless, it may not sufficiently reflect employee performance.

Lastly, Vietnam shares many common features with other developing countries such as weak accountability, limited capacity, collectivism, nepotism and the effect of personal ties on workplaces. For this reason, practitioners and policy-makers in developing contexts can refer to the findings and lessons from this research. However, it is worth noting that Vietnam also has distinct characteristics in terms of historical legacies and political institutions. Therefore, the suggestions in this research should be considered with caution.

\subsection{Recommendations for future research}

This research has some suggestions for future research.

First, this is one of the first studies that explore the effect of agency accountability, HR autonomy, entrepreneurial leadership and HR competence on the development of employee PM in the public sector in transitional and developing countries. Further studies should duplicate these contextual variables and possibly expand other contextual variables such as the extent of corruption, job types, organisational size and political environment in other developing settings.

Second, whenever possible, the overall system effectiveness (i.e. organisational performance and employee performance) should be measured by objective or secondary data such as the PAR index, customer satisfaction and the ratings of direct supervisors rather than by employees' perceptions,. It is also suggested that future studies use longitudinal surveys with random samples to enhance the validity of generalizations.

Third, as an emerging and transitional economy, Vietnam has initiated many ambitious PAR programmes such as reforming institution, reforming the organisation of the state administrative apparatus, enhancing the quality of the contingent of public employees, and reforming public finance. However, the research on PAR and HRM in the public sector in this 
country is relatively scarce. Therefore, investigating the factors contributing to the success as well as failure will be very useful for Vietnam and other nations sharing a similar context.

Fourth, while this research finds that communication and training effectively support the introduction of employee PM, other effective intervention practices have not been sufficiently investigated. This research suggests that there is still a gap in the literature in examining the effectiveness of other intervention practices such as using IT and outside consulting services. A survey conducted in the United States showed that up to 17 percent of employee PM systems were developed by external consultants (Gorman, Meriac et al. 2017). Meanwhile, in 2012, about 71 percent of U.S companies were using web-based PM systems (Lawler, Benson et al. 2012). Therefore, examining the impact of these intervention practices on the successful adoption of employee PM will be valuable for both developed and developing countries.

Finally, the interviewees often revealed that their organisations were very confused in developing performance standards or key performance indicators (KPIs) for white-collar employees particularly for those who work in policy-making agencies (coping jobs). This is because most of their work "produces no tangible, countable end product" (Boyett and Conn 1988, p.209). In this regard, Cardona (2006 p3), concludes that, "Measurement of performance, particularly in areas where there are no obvious quantifiable outputs, is a very difficult issue." Hasnain, Manning et al. (2012) conclude that while much of the research on employee PM has centred on craft (measurable) jobs, very little high-quality research has been done on coping (not easily measurable) jobs. Therefore, future research into employee PM in policy-making agencies with coping jobs is highly recommended.

\subsection{Conclusion of the thesis}

Given that government activities are highly personnel-intensive, managing public employee performance is recognised as central to enhance the quality of the public service (Ingraham and Kneedler 2000). This need is even more urgent in developing and transitional countries where poor performance has made several socio-economic development programmes and PAR initiatives less effective or fail (Taylor 1992; Randma-Liiv 2005; Ho and Im 2015).

Supported by management theories and the doctrine of NPM, employee PM supposedly influences individual attitudes and performance, and subsequently organisational performance (De Waal and Gerritsen-Medema 2006; DeNisi and Sonesh 2011). However, the literature has documented a high failure rate of employee PM innovating projects. There is a suspicion that the introduction of employee PM might not be compatible in developing countries because of 
problems of institutions, culture and capacity. This research was conducted to shed light on the introduction of employee PM in transitional and developing countries.

By adopting mixed methods and using the context of Vietnam as a transitional country, this research finds that employee PM is applicable in developing and transitional countries. Whatever the challenges in these countries are, it is clear that having more advanced employee PM practices will contribute to producing better outcomes, including improved job attitudes and organisational performance. The research also shows that the development of employee PM is driven by three contextual factors, namely agency accountability, HR autonomy, and entrepreneurial leadership. HR competence can only promotes the development of employee PM if entrepreneurial leadership is present.

This research also finds that the effects of HR autonomy and entrepreneurial leadership are weakened by nepotism and interpersonal relationships while they are strengthened by communication and training. However, neither cultural variables nor intervention variables moderates the agency accountability-employee PM relationship. This implies that while other contextual factors discussed in this research are important, increased accountability is a fundamental and stable factor to strengthen the development of employee PM and improve the performance of public organisations.

The findings from this research provide a number of meaningful suggestions for policymakers and practitioners. Accordingly, effective strategies for developing employee PM include strengthening accountability for public organisations, fostering entrepreneurial leadership, decentralising HRM authority and consolidating the capacity of HR units. Besides these strategic solutions, specific solutions include using goal-based appraisal criteria, holding raters accountable for rating results, rewarding employees according to individual and team performance, transferring poor performers, encouraging employee participation, enhancing awareness of employee PM, and providing training for users.

Despite making important contributions, this research has a number of limitations regarding sampling, measuring and research scope. Further studies are suggested to duplicate this study in other developing contexts, using random samples and objective measures to examine if the initial findings of this study are corroborated. 


\section{References}

Abbas, M. Z. (2014). "Effectiveness of performance appraisal on performance of employees." Journal of Business and Management 16(6): 173-178.

Abdalla, H. F., et al. (1998). "Assessing the perceptions of human resource managers toward nepotism: a cross-cultural study." International Journal of Manpower 19(8): 554-570.

Acuña-Alfaro, J. (2009). "Reforming public administration in Vietnam: Current situation and recommendations." United Nations Development Programme (UNDP), Vietnam Fatherland Front (VFF), Centre for Community Support Development Studies (CECODES). Ha Noi: National Politics Publishing House.

Acuña-Alfaro, J. and A. Tran (2016). "Vietnam's public bureaucracy and implementation of two ASEAN policies." The Role of the Public Bureaucracy in Policy Implementation in Five ASEAN Countries 9: 370.

Adamowki, S., et al. (2007). "The autonomy gap: barriers to effective school leadership." Thomas B. Fordham Foundation \& Institute, NY.

Adams, J. S. (1963). "Towards an understanding of inequity." The Journal of Abnormal and Social Psychology 67(5): 422.

Adams, J. S. and W. B. Rosenbaum (1962). "The relationship of worker productivity to cognitive dissonance about wage inequities." Journal of Applied Psychology 46(3): 161.

Ågerfalk, P. J. (2013). "Embracing diversity through mixed methods research." European Journal of Information systems 22(3): 6.

Aguinis, H. (2009). Performance management, Pearson Prentice Hall Upper Saddle River, NJ.

Agyemang, G. and B. Ryan (2013). Accountability and performance management systems within private and public sector organisational change processes. Managing Reality: Accountability and the Miasma of Private and Public Domains, Emerald Group Publishing Limited: 1-38.

Ahenkan, A., et al. (2018). "Performance management implementation challenges in Ghana's local government system: evidence from the Sefwi Wiawso Municipal Assembly." International journal of productivity and performance management 67(3): 519-535.

Akbar, R. (2011). Performance measurement and accountability in Indonesian Local Government, Dotoral Dissertation, Curtin University.

Akbar, R., et al. (2012). "Performance measurement in Indonesia: the case of local government." Pacific Accounting Review 24(3): 262-291.

Allan, P. (1994). "Designing and implementing an effective performance appraisal." Review of Business 16(2): 3 .

Anderson, J. C. and D. W. Gerbing (1988). "Structural equation modeling in practice: A review and recommended two-step approach." Psychological bulletin 103(3): 411.

Andersson, U., et al. (2014). "From the editors: Explaining interaction effects within and across levels of analysis." Journal of international business studies 45(9): 1063-1071. 
Anh, V. T. T. (2016). "Vietnam: decentralization amidst fragmentation." Journal of Southeast Asian Economies (JSEAE) 33(2): 188-208.

Anjum, A., et al. (2011). "Performance appraisal systems in public sector universities of Pakistan." International Journal of Human Resource Studies 1(1): Pages 41-51.

Arabie, P. (1994). Cluster analysis in marketing research, in advanced methods of marketing research. Cambridge, MA: Blackwell Publishers.

Arasli, H. and M. Tumer (2008). "Nepotism, Favoritism and Cronyism: A study of their effects on job stress and job satisfaction in the banking industry of north Cyprus." Social Behavior and Personality: an international journal 36(9): 1237-1250.

Armenakis, A. A. and A. G. Bedeian (1999). "Organizational change: A review of theory and research in the 1990s." Journal of management 25(3): 293-315.

Armenakis, A. A. and S. G. Harris (2002). "Crafting a change message to create transformational readiness." Journal of organizational change management 15(2): 169-183.

Armstrong, M. (2002). Employee reward. London, CIPD Publishing.

Armstrong, M. (2006). Performance management: Key strategies and practical guidelines. Philadelphia, Kogan Page.

Armstrong, M. and A. Baron (1998). Performance management: the new realities. London, Institute of Personnel and Development.

Armstrong, M. and A. Baron (2005). Managing performance: performance management in action. London, CIPD publishing.

Arnaboldi, M., et al. (2015). "Performance Management in the Public Sector: The Ultimate Challenge." Financial Accountability \& Management 31(1): 1-22.

Ashford, S. J. and L. L. Cummings (1983). "Feedback as an individual resource: Personal strategies of creating information." Organizational behavior and human performance 32(3): 370-398.

Asim, M. (2001). "Performance appraisal in the Maldives public service: challenges and issues." Public Administration and Development 21(4): 289-296.

Askim, J., et al. (2015). "Accountability and performance management: The Norwegian hospital, welfare, and immigration administration." International Journal of Public Administration 38(13-14): 971-982.

Aycan, Z. (2005). "The interplay between cultural and institutional/structural contingencies in human resource management practices." The International Journal of Human Resource Management 16(7): 1083-1119.

Aycan, Z., et al. (2000). "Impact of culture on human resource management practices: A 10-country comparison." Applied psychology 49(1): 192-221.

Aycan, Z., et al. (1999). "Organizational Culture and Human Resource Management Practices The Model of Culture Fit." Journal of Cross-Cultural Psychology 30(4): 501-526. 
Azzone, G. and T. Palermo (2011). "Adopting performance appraisal and reward systems: A qualitative analysis of public sector organisational change." Journal of organizational change management 24(1): $90-111$.

BacGiang (2013). Report on piloting appraisal of chief executives. Bacgiang, Vietnam, Bacgiang Department of Home Affairs.

Bach, D. (2016). GDP increases 6.21 percent, the income per capita achieves \$2.215. Vietnam economy, Retrieved from: http://vneconomy.vn.

Baron, R. M. and D. A. Kenny (1986). "The moderator-mediator variable distinction in social psychological research: Conceptual, strategic, and statistical considerations." Journal of personality and social psychology 51(6): 1173.

Bawole, J. N., et al. (2013). "Performance appraisal or praising performance? The culture of rhetoric in performance management in Ghana civil service." International Journal of Public Administration 36(13): 953-962.

Beardwell, J. and T. Claydon (2007). Human resource management: a contemporary approach. London, Pitman.

Behery, M. H. and R. A. Paton (2008). "Performance appraisal-cultural fit: organizational outcomes within the UAE." Education, Business and Society: Contemporary Middle Eastern Issues 1(1): 34-49.

Berberian, J. N. (2008). The impact of a non-monetary reward program on employee job satisfaction, Dotoral Dessertation, Pepperdine University.

Bercovitz, J. and M. Feldman (2004). "Academic entrepreneurs: Social learning and participation in university technology transfer." Work in progress, University of Toronto.

Berman, E. M. (2015). "HRM in development: lessons and frontiers." Public Administration and Development 35(2): 113-127.

Berman, E. M., et al. (2015). Human resource management in public service: Paradoxes, processes, and problems, London, Sage Publications.

Berman, E. M., et al. (2017). "Executive Entrepreneurship in National Departments." Administration \& Society: 1-30.

Berrell, M., et al. (1999). "The influence of culture on managerial behaviour." Journal of Management Development 18(7): 578-589.

Bhattacherjee, A. (2012). Social science research: Principles, methods, and practices. University of Georgia, USA, Open Access Textbook.

Bhuiyan, S. H. and F. Amagoh (2011). "Public sector reform in Kazakhstan: issues and perspectives." International Journal of Public Sector Management 24(3): 227-249.

Bilgin, K. U. (2007). "Performance management for public personnel: multi-analysis approach toward personnel." Public Personnel Management 36(2): 93-113.

Birkinshaw, J., et al. (2008). "Management innovation." Academy of management review 33(4): 825845 . 
Biron, M., et al. (2011). "Performance management effectiveness: lessons from world-leading firms." The International Journal of Human Resource Management 22(06): 1294-1311.

Bititci, U. S., et al. (1997). "Integrated performance measurement systems: a development guide." International journal of operations \& production management 17(5): 522-534.

Blacklock, K. (2002). "Dealing with an Incompetent Teacher." Principal 81(4): 26-28.

Boice, D. F. and B. H. Kleiner (1997). "Designing effective performance appraisal systems." Work study 46(6): 197-201.

Bordia, P., et al. (2004). "Uncertainty during organizational change: Types, consequences, and management strategies." Journal of business and psychology 18(4): 507-532.

Boselie, P., et al. (2005). "Commonalities and contradictions in HRM and performance research." Human Resource Management Journal 15(3): 67-94.

Bouckaert, G. and J. Halligan (2007). Managing performance: International comparisons. New York, Routledge.

Bouckenooghe, D., et al. (2009). "Organizational change questionnaire-climate of change, processes, and readiness: Development of a new instrument." the Journal of Psychology 143(6): 559-599.

Bowman, J. S. (2010). "The success of failure: the paradox of performance pay." Review of Public Personnel Administration 30(1): 70-88.

Boyd, B. K., et al. (1993). "Divergence between archival and perceptual measures of the environment: Causes and consequences." Academy of management review 18(2): 204-226.

Boyett, J. H. and H. P. Conn (1988). "Developing White-Collar Performance Measures." Global Business and Organizational Excellence 7(3): 209-218.

Bozionelos, N. and L. Wang (2007). "An investigation on the attitudes of Chinese workers towards individually based performance-related reward systems." The International Journal of Human Resource Management 18(2): 284-302.

Bratton, J. and J. Gold (2012). Human resource management: theory and practice. London, Palgrave Macmillan.

Bretz Jr, R. D., et al. (1992). "The current state of performance appraisal research and practice: Concerns, directions, and implications." Journal of management 18(2): 321-352.

Bridges, E. M. (1985). "Managing the Incompetent Teacher-What Can Principals Do?" NASSP Bulletin 69(478): 57-65.

Bridges, E. M. (2013). The incompetent teacher: Managerial responses. London and New York, Routledge.

Brooks, J. and N. King (2014). Doing template analysis: evaluating an end of life care service. London, Sage publication.

Brooks, J., et al. (2015). "The utility of template analysis in qualitative psychology research." Qualitative Research in Psychology 12(2): 202-222. 
Brown, A. (2005). "Implementing performance management in England's primary schools." International journal of productivity and performance management 54(5/6): 468-481.

Brown, M., et al. (2010). "Consequences of the performance appraisal experience." Personnel Review 39(3): 375-396.

Bryman, A. (2008). Social research methods, Oxford university press.

Bryman, A. (2015). Social research methods, Oxford university press.

Bui, V. T. (2011). The PAR has not yet met the requirement. Paper presented at the conference on Public administration reform in Vietnam under the lens of academics. Hanoi, Vietnam.

Burgess, S. and M. Ratto (2003). "The role of incentives in the public sector: Issues and evidence." Oxford review of economic policy 19(2): 285-300.

Burns, J. P. and W. Xiaoqi (2010). "Civil service reform in China: Impacts on civil servants' behaviour." The China Quarterly 201: 58-78.

Burns, J. P. and Z. Zhiren (2010). "Performance management in the government of the People's Republic of China: accountability and control in the implementation of public policy." OECD Journal on Budgeting 10(2): 7.

Bute, M. (2011). "The effects of nepotism and favoritism on employee behaviors and human resources practices: a research on Turkish public banks." TODAĐE's Review of Public Administration 5(2): 158208.

Büte, M. (2011). "Perceived nepotism and its relation to job satisfation, negative word of mouth and intention to quite." Electric Journal of Social Science 10(36): 187-203.

Byrne, B. M. (2016). Structural equation modeling with AMOS: Basic concepts, applications, and programming. New York, Routledge.

Cantarelli, P., et al. (2016). "A meta-analysis of job satisfaction correlates in the public administration literature." Review of Public Personnel Administration 36(2): 115-144.

Carassus, D., et al. (2014). "Factors that Determine or Influence Managerial Innovation in Public Contexts: The Case of Local Performance Management." Public Organization Review 14(2): 245-266.

Cardona, F. (2006). "Performance Related Pay in the Public Service in OECD and EU Member States." Programme SIGMA de l'OCDE. Paris.

Caruth, D. L. and J. H. Humphreys (2008). "Performance appraisal: essential characteristics for strategic control." Measuring Business Excellence 12(3): 24-32.

Cavalluzzo, K. S. and C. D. Ittner (2004). "Implementing performance measurement innovations: evidence from government." Accounting, Organizations and Society 29(3): 243-267.

Cerasoli, C. P., et al. (2014). "Intrinsic motivation and extrinsic incentives jointly predict performance: A 40-year meta-analysis." Psychological bulletin 140(4): 980.

Chang, E. and J. Hahn (2006). "Does pay-for-performance enhance perceived distributive justice for collectivistic employees?" Personnel Review 35(4): 397-412. 
Chawla, A. and E. Kevin Kelloway (2004). "Predicting openness and commitment to change." Leadership \& Organization Development Journal 25(6): 485-498.

Chen, C. C., et al. (2004). "Guanxi practices and trust in management: A procedural justice perspective." Organization Science 15(2): 200-209.

Chen, Y., et al. (2009). "Supervisor-Subordinate Guanxi: Developing a Three-Dimensional Model and Scale." Management and organization Review 5(3): 375-399.

Cheng, B.-S., et al. (2002). "Guanxi, zhongcheng, competence and managerial behavior in the Chinese context." Journal of Chinese psychology 44(2): 151-166.

Cheng, M.-I., et al. (2006). "Implementing a new performance management system within a projectbased organization: A case study." International journal of productivity and performance management 56(1): $60-75$.

Cheung, A. B., et al. (2013). "Institutionalizing performance management in Asia: looking East or West?" International Journal of Public Sector Management 26(5): 347-360.

Cheung, M. F., et al. (2009). "Supervisor-subordinate guanxi and employee work outcomes: The mediating role of job satisfaction." Journal of Business Ethics 88(1): 77-89.

Chiang, F. F. and T. A. Birtch (2010). "Appraising Performance across Borders: An Empirical Examination of the Purposes and Practices of Performance Appraisal in a Multi-Country Context." Journal of Management Studies 47(7): 1365-1393.

Chin, W. W. (1998). "Commentary: Issues and opinions on structural equation modeling." MIS quarterly 22(1): 7-16.

Cho, Y. J. and J. W. Lee (2011). "Performance management and trust in supervisors." Review of Public Personnel Administration 32(3): 236-259.

Christensen, T., et al. (2008). "Administrative reform in China's central government-how muchlearning from the West'?" International Review of Administrative Sciences 74(3): 351-371.

Cierco, T. (2013). "Public administration reform in Macedonia." Communist and Post-Communist Studies 46(4): 481-491.

Ciobanu, A. and B. Ristea (2015). "The relationship between performance appraisal and civil servants' motivation." Management Research and Practice 7(2): 5-19.

Clampitt, P. G. and C. W. Downs (1993). "Employee perceptions of the relationship between communication and productivity: A field study." The Journal of Business Communication 30(1): 5-28.

Cleveland, J., et al. (1986). Rater intentions in appraisal ratings: Malevolent manipulation or functional fudging. First annual conference of the Society for Industrial and Organizational Psychology, Chicago.

Coens, T. and M. Jenkins (2002). Abolishing performance appraisals: Why they backfire and what to do instead, San Francisso, CA, Berrett-Koehler Publishers.

Cohen, J. and P. Cohen (1975). Applied multiple regression/correlation analysis for the behavioral sciences. New York, Routledge. 
Colli, L. A. (2013). The performance management system of the Department of Economic Development and Tourism (Western Cape), Doctoral dissertation, Cape Peninsula University of Technology.

Colton, D. and R. W. Covert (2007). Designing and constructing instruments for social research and evaluation. San Francisco, CA, John Wiley \& Sons.

Conger, J., et al. (1998). "CEO appraisals: Holding corporate leadership accountable." Organizational Dynamics 27(1): 7-20.

Cooke, F. L. (2003). "Seven reforms in five decades." Journal of the Asia Pacific Economy 8(3): 380404.

Corbetta, P. (2003). Social research: Theory, methods and techniques. London, Sage.

Cornell, J. E. and S. M. Herman (1989). "Change, or get changed." Organization Development Journal 7(4): 76-81.

CPV (2011). The resolution of the 11th national congress of the comunist party of Vietnam on socioeconomic development strategy, The communist party of Vietnam.

CPV (2012). The urgent problems in developing the Party. Resolution No 12, the fourth meeting, the

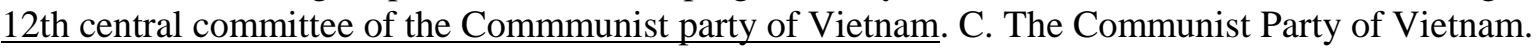

CPV (2016). Evaluation on the outcomes of implementing the socio-economic tasks in the period 20112015 and the plan of the socio-economic tasks in the period 2016-2020, The Communist Party of Vietnam.

CPV (2016). Evaluation report on the result of five year socioeconomic plan (2011 - 2015) and the goals and tasks for the priod of 2016 - 2020) Communist Party of Vietnam.

CPV (2016). Strengthening building and rectifying the party, preventing the depravation in political ideology, morality, lifestyle and the manifestation of internal transformation. Resolution No 4th, the 12th central committee of the Communist of Vietnam. C. The Communist Party of Vietnam.

CPV (2018). Resolution no 7, the 12th Central Committee of the Communist Party of Vietnam on developing a contingent of cadres at all levels, particularly the strategic level. 26-NQ/TW. C. The Communist Party of Vietnam.

CPV, C. P. o. V. (1997). The Cadre Strategy for the Period of Speeding up Industrialization and Modernization, Communist Party of Vietnam.

Crampton, S. M. and J. A. Wagner III (1994). "Percept-percept inflation in microorganizational research: An investigation of prevalence and effect." Journal of Applied Psychology 79(1): 67.

Creswell, J. W. (2005). Educational research: Planning, conducting, and evaluating quantitative and qualitative research, Prentice Hall Upper Saddle River, NJ.

Creswell, J. W. (2009). Research design: Qualitative, quantitative, and mixed methods approaches. Thousand Oaks, CA, Sage publications.

Creswell, J. W. (2014). Research design: Qualitative, quantitative, and mixed methods approaches. Thousand Oaks, CA, Sage publications. 
Creswell, J. W. and V. L. P. Clark (2007). Designing and conducting mixed methods research. Thousand Oaks, CA, Sage.

Creswell, J. W. and A. Tashakkori (2007). Developing publishable mixed methods manuscripts, Los Angeles, CA, Sage.

Cunha, M. P. e., et al. (2018). "Why does performance management not perform?" International journal of productivity and performance management 67(4): 673-692.

Daley, D. M. (1992). Performance appraisal in the public sector: Techniques and applications. Westport, Connecticut, Quorum Books.

Damiran, T. and R. Pratt (2008). "Public Administration Development and Reform in a Post-Communist Regime: The Case of Mongolia." Asia Pacific Journal of Public Administration 30(2): 193-216.

Danang, D. o. H. A. (2014). Reporting outcomes of the pilot scheme on innovating performance appraisal in Danang. Danang Department of Home Affairs, Vietnam.

Dao, T. T. T. (2015). Appraising public employees based on public service performance. Department of Administration, Doctoral Dissertation, National Academy of Public Administration.

Davis, B. (2015). Best practices in the field of performance management: A Delphi study, Doctoral Dissertation, The University of the Rockies.

Dawis, R. V. (2004). "Job satisfaction." Comprehensive handbook of psychological assessment 4: 470481.

Dawson, T. C. and K. L. Billingsley (2000). Unsatisfactory Performance: How California's K-12 Education System Protects Mediocrity and How Teacher Quality Can Be Improved. San Francisco, Pacific Research Institute for Public Policy.

De Waal, A. and G. Gerritsen-Medema (2006). "Performance management analysis: a case study at a Dutch municipality." International journal of productivity and performance management 55(1): 26-39.

De Waal, A. A. (2007). "Is performance management applicable in developing countries? The case of a Tanzanian college." International journal of emerging markets 2(1): 69-83.

Deci, E. L. and R. M. Ryan (2000). "The" what" and" why" of goal pursuits: Human needs and the selfdetermination of behavior." Psychological inquiry 11(4): 227-268.

Decramer, A., et al. (2012). "External pressures affecting the adoption of employee performance management in higher education institutions." Personnel Review 41(6): 686-704.

DeNisi, A. and C. E. Smith (2014). "Performance Appraisal, Performance Management, and Firm-Level Performance: A Review, a Proposed Model, and New Directions for Future Research." The Academy of Management Annals 8(1): 127.

DeNisi, A. S. and K. R. Murphy (2017). "Performance appraisal and performance management: 100 years of progress?" Journal of Applied Psychology 102(3): 421.

DeNisi, A. S. and R. D. Pritchard (2006). "Performance appraisal, performance management and improving individual performance: A motivational framework." Management and organization Review 2(2): 253-277. 
DeNisi, A. S. and S. Sonesh (2011). The appraisal and management of performance at work. Washington, American Psychological Association.

Denkyira, F. O. (2014). "Establishing effective performance appraisal practices in the Ghana Civil Service." Available at https://papers.ssrn.com/sol3/papers.cfm?abstract id=2394578.

Dess, G. G. and R. B. Robinson (1984). "Measuring organizational performance in the absence of objective measures: the case of the privately-held firm and conglomerate business unit." Strategic management journal 5(3): 265-273.

Dessler, G. (2008). Human resource management, Prentice Hall USA.

DeVries, D. L., et al. (1981). Performance appraisal on the line. Greensboro, NC, Center for Creative Leadership.

Diep, B. (2012). GDP percapita in Vietnam has increased 10 times over 20 years. Dantri, Retrieved from: www.dantri.com.vn.

Diep, S. V. (2011). The evaluation of the PAR programme (2001 - 2010) through the lens of an expert. Paper presented at the conference on Public administration reform in Vietnam under the lens of academics. Ha Noi, Vietnam.

Doan, N. (2018). Why public employees in Danang resign. Zing.vn, Retrieved from: https://news.zing.vn/.

Donahue, A. K., et al. (2000). "Measuring government management capacity: A comparative analysis of city human resources management systems." Journal of Public Administration Research and Theory 10(2): 381-412.

Dou, D., et al. (2016). "The effects of autonomy gap in personnel policy, principal leadership and teachers' self-efficacy on their organizational commitment." Asia Pacific Education Review 17(2): 339353.

Dowling, B. and R. Richardson (1997). "Evaluating performance-related pay for managers in the National Health Service." International Journal of Human Resource Management 8(3): 348-366.

Drechsler, W. (2000). Public administration in Central and Eastern Europe: Considerations from the "state science" approach. Northampton, Massachusetts, Edward Elgar.

Dubois, D. and W. Rothwell (2004). Competency-Based Human Resource Management: Discover a New System for Unleashing the Productive Power of Exemplary Performers. Palo Alto, CA, DavisBlack.

Duong, B. (2017). Vietnam's public debt reaches \$ 81 billion. VNeconomy, Retrieved from: http://vneconomy.vn.

Dzimbiri, L. B. (2008). "Experiences in new public management in Africa: the case of performance management systems in Botswana." Africa Development 33(4): 43-58.

Easterby-Smith, M., et al. (2012). Management research. Thousand Oaks, CA, Sage.

Ehrgott, R. (1993). A Study of the Marginal Teacher in California. Paper presented at the annual meeting of the California Educational Research Association. San Francisco, CA. 
Eisenberg, R., et al. (1990). "Perceived organizational support and employee diligence, commitment, and innovation." Journal of Applied Psychology 75(1): 51-59.

Elving, W. J. (2005). "The role of communication in organisational change." Corporate Communications: An International Journal 10(2): 129-138.

Emerson, B. (2002). "Training for performance measurement success." Government Finance Review 18(2): $22-22$.

Entrekin, L. and Y. W. Chung (2001). "Attitudes towards different sources of executive appraisal: A comparison of Hong Kong Chinese and American managers in Hong Kong." International Journal of Human Resource Management 12(6): 965-987.

Erdogan, B. (2003). "Antecedents and consequences of justice perceptions in performance appraisals." Human Resource Management Review 12(4): 555-578.

Eun, J. (2010). "Public Accountability in Collaborative Governance: Lessons from Korean Community Centers." Korean Journal of Policy studies 25(1): 143-173.

Evans, E. M. and S. L. McShane (1988). "Employee perceptions of performance appraisal fairness in two organizations." Canadian Journal of Behavioural Science/Revue canadienne des sciences du comportement 20(2): 177.

Farashahi, M., et al. (2005). "Institutionalized norms of conducting research and social realities: a research synthesis of empirical works from 1983 to 2002." International Journal of Management Reviews 7(1): 1-24.

Farazmand, A. (2002). Administrative reform in developing nations, Santa Barbara, CA, Greenwood Publishing Group.

Fernandez, S. and H. G. Rainey (2006). "Managing successful organizational change in the public sector." Public Administration Review 66(2): 168-176.

Ferris, G. R., et al. (2008). "The performance evaluation context: Social, emotional, cognitive, political, and relationship components." Human Resource Management Review 18(3): 146-163.

Field, A. (2009). Discovering statistics using SPSS, Thousand Oaks, CA, Sage publications.

Fisher, R. J. (1993). "Social desirability bias and the validity of indirect questioning." Journal of consumer research 20(2): 303-315.

Fletcher, C. (2001). "Performance appraisal and management: The developing research agenda." Journal of Occupational and Organizational Psychology 74(4): 473-487.

Fletcher, C. and R. Williams (1996). "Performance management, job satisfaction and organizational commitment1." British Journal of Management 7(2): 169-179.

Ford, R. and F. McLaughlin (1986). "Nepotism: Boon or bane." Personnel Administrator 31(11): 78-89.

Fornell, C. and D. F. Larcker (1981). "Evaluating structural equation models with unobservable variables and measurement error." Journal of marketing research 18(1): 39-50. 
Fuchs, C. and A. Diamantopoulos (2009). "Using single-item measures for construct measurement in management research: conceptual issues and application guidelines." Die Betriebswirtschaft 69(2): 195210.

Furnham, A. and B. Gunter (1993). Corporate assessment: Auditing a company's personality. London, Routledge.

Gabrenya Jr, W. K. and K.-K. Hwang (1996). Chinese social interaction: Harmony and hierarchy on the good earth. New York, US, Oxfort University Press.

Galang, M. C. (1999). "Stakeholders in high-performance work systems." International Journal of Human Resource Management 10(2): 287-305.

Gallivan, M. J. (2001). "Meaning to change: How diverse stakeholders interpret organizational communication about change initiatives." IEEE Transactions on Professional Communication 44(4): 243-266.

Gardner, C. (2008). "Employee evaluation: is it worth the effort?" DVM Magazine, January 1: 4F.

Garnett, J. L., et al. (2008). "Penetrating the performance predicament: communication as a mediator or moderator of organizational culture's impact on public organizational performance." Public Administration Review 68(2): 266-281.

Gavish, B., et al. (2000). "Performance-based reward distribution methods for anonymous decisionmaking groups." Group Decision and Negotiation 9(5): 393-413.

Gefen, D., et al. (2000). "Structural equation modeling and regression: Guidelines for research practice." Communications of the association for information systems 4(1): 1-78.

Gerrish, E. (2016). "The impact of performance management on performance in public organizations: A meta-analysis." Public Administration Review 76(1): 48-66.

Gibbs, G. R. (2008). Analysing qualitative data. Thousand Oaks, CA, Sage.

Gibson, C. B. and J. Birkinshaw (2004). "The antecedents, consequences, and mediating role of organizational ambidexterity." Academy of management journal 47(2): 209-226.

Glendinning, P. M. (2002). "Performance management: Pariah or messiah." Public Personnel Management 31(2): 161-178.

Goh, S. C. (2012). "Making performance measurement systems more effective in public sector organizations." Measuring Business Excellence 16(1): 31-42.

Gorman, C. A., et al. (2017). "An exploratory study of current performance management practices: Human resource executives' perspectives." International Journal of Selection and Assessment 25(2): 193-202.

Gorman, C. A. and J. R. Rentsch (2009). "Evaluating frame-of-reference rater training effectiveness using performance schema accuracy." Journal of Applied Psychology 94(5): 1336.

Gosling, S. D., et al. (2004). "Should we trust web-based studies? A comparative analysis of six preconceptions about internet questionnaires." American psychologist 59(2): 93.

Government (2016) Why the Prime Minister requests "looking for talented persons not for relatives". 
Greenberg, J. (1986). "Determinants of perceived fairness of performance evaluations." Journal of Applied Psychology 71(2): 340.

Greenberg, J. (1987). "A taxonomy of organizational justice theories." Academy of management review 12(1): 9-22.

Gregory, R. (2016). "Combating corruption in Vietnam: a commentary." Asian Education and Development Studies 5(2): 227-243.

GSO (2016). Press release on the current situation of socioeconomic development, General Statistics Office of Vietnam.

Gu, F. (2013). A comparative study of performance appraisal system in three banks in contemporary China: the role of guanxi in appraisals, Doctoral Dissertation, The London School of Economics and Political Science (LSE).

Guba, E. and Y. Lincoln (2005). Paradigmatic Controversies, contradictions and emerging confluences (3rd ed). Thousand Oaks, CA, Sage Publications.

Ha, N. Q. (2013). The current performance appraisal in Vietnam. Public administration reform. Retrieved from: http://caicachhanhchinh.gov.vn.

Ha, N. Q., et al. (2011). Innovating the performance appraisal method for civil servants in public organizations. Hanoi, Vietnam, Vietnamese Ministry of Home Affairs.

Habermas, J. (1984). "The theory of communicative action, volume I." Boston: Beacon.

Haines, C. A. (2011). Value added by mixed methods research: A multiphase mixed methods design, The University of Nebraska-Lincoln.

Haines III, V. Y. and S. St-Onge (2012). "Performance management effectiveness: practices or context?" The International Journal of Human Resource Management 23(6): 1158-1175.

Hair, J. F., et al. (2006). Multivariate data analysis (6th). Upper Saddle River, NJ: Pearson Prentice Hall.

Hair, J. F., et al. (2012). "An assessment of the use of partial least squares structural equation modeling in marketing research." Journal of the Academy of Marketing Science 40(3): 414-433.

Han, J., et al. (2006). "The HR competencies-HR effectiveness link: A study in Taiwanese high-tech companies." Human Resource Management 45(3): 391-406.

Hansson, J., et al. (2003). "Managing commitment: increasing the odds for successful implementation of TQM, TPM or RCM." International Journal of Quality \& Reliability Management 20(9): 993-1008.

Harbi, S. A., et al. (2017). "Culture, Wasta and perceptions of performance appraisal in Saudi Arabia." The International Journal of Human Resource Management 28(19): 2792-2810.

Hardwick, J. and G. Winsor (2002). RCM-Making the Process More Cost Effective One Year Later. Proceedings of International Conference on Maintenance Societies (ICOMS), Brisbane, Australia.

Harris, M. M. (1994). "Rater motivation in the performance appraisal context: A theoretical framework." Journal of management 20(4): 735-756. 
Hasnain, Z., et al. (2012). Performance-related pay in the public sector: a review of theory and evidence, The World Bank.

Hayajenh, A. F., et al. (1994). "Research note: Assessing the effect of nepotism on human resource managers." International Journal of Manpower 15(1): 60-67.

Hayajneh, A. F., et al. (1994). "Nepotism as a dilemma for managing human resources overseas: Its impact on employees, management and organizations." Journal of Transnational Management Development 1(1): 51-73.

Heathfield, S. (2007). "Performance appraisals don't work - what does?" The Journal for Quality and Participation 30(1): 6-9.

Heinrich, C. J. and G. Marschke (2010). "Incentives and their dynamics in public sector performance management systems." Journal of Policy Analysis and Management 29(1): 183-208.

Hesse, J. J. (1993). "From transformation to modernization: Administrative change in Central and Eastern Europe." Public administration 71(1-2): 219-257.

Heyel, C. (1958). Appraising executive performance. New York, American Management Association.

Ho, A. T.-K. and T. Im (2015). "Challenges in building effective and competitive government in developing countries: An institutional logics perspective." The American Review of Public Administration 45(3): 263-280.

Hoai, T. (2017). Hundred public employees leave public organizations: public employees' salary is lower than those of security guards. Vietnam.net, Retrieved from: http://vietnam.net.

Hoang, T. B. (2014). The danger of nepotism, bribes, and personal relationships in personnel practice. Dantri. Hanoi, Retrieved from: www.dantri.com.vn.

Hofstede, G. (1984). Culture's consequences: International differences in work-related values. Newbury Park, CA, sage.

Hofstede, G. (1993). "Cultural constraints in management theories." The Academy of Management Executive 7(1): 81-94.

Hofstede, G. (1998). "Think locally, act globally: Cultural constraints in personnel management." Management and International Review 38(2): 7-26.

Hofstede, G. (2001). Culture's consequences: Comparing values, behaviors, institutions and organizations across nations, Thousand Oaks, CA, Sage.

Hofstede, G. (2007). "Asian management in the 21st century." Asia Pacific Journal of Management 24(4): 411-420.

Hofstede, G., et al. (1990). "Measuring organizational cultures: A qualitative and quantitative study across twenty cases." Administrative science quarterly 23(2): 286-316.

Hoi, T. (2013). Not easy to lay off public officials. Kienthuc. Vietnam, Retrieved from: http://kienthuc.net.vn.

Holloway, J., et al. (2009). "Lessons learned from performance management systems implementations." International journal of productivity and performance management 58(4): 367-390. 
Hood, C. (1991). "A public management for all seasons?" Public administration 69(1): 3-19.

Hooper, D., et al. (2008). "Structural equation modelling: Guidelines for determining model fit." Electronic Journal of Business Research Methods 6(1): 53-60.

Hope Sr, K. R. (2003). "Employee perceptions of leadership and performance management in the Botswana public service." Public Personnel Management 32(2): 301-313.

Horgan, J. and P. Muhlau (2003). "The adoption of high performance human resource practices in Ireland: An integration of contingency and institutional theory." Irish Journal of Management 24(1): 2647.

Hu, L. t. and P. M. Bentler (1999). "Cutoff criteria for fit indexes in covariance structure analysis: Conventional criteria versus new alternatives." Structural equation modeling: a multidisciplinary journal 6(1): $1-55$.

Huang, J. (2006). Factionalism in Chinese communist politics, Cambridge University Press, NY.

Hulin, C. L. (1982). "Some reflections on general performance dimensions and halo rating error." Journal of Applied Psychology 67(2): 165-170.

Huynh, T. V. (2011). Public employee training: from the concept to action. Paper presented at the conference on Public administration reform in Vietnam through the lens of academics. Hanoi, Vietnam.

Ichniowski, T. (1988). "The new nepotism: why dynasties are making a comeback." Business Week 31(4): 106-109.

Igbaria, M., et al. (1997). "Personal computing acceptance factors in small firms: a structural equation model." MIS quarterly: 279-305.

Ilgen, D. R., et al. (1993). "Performance appraisal process research in the 1980s: what has it contributed to appraisals in use?" Organizational Behavior and Human Decision Processes 54(3): 321-368.

Ilgen, D. R. and J. M. Feldman (1983). "Performance appraisal: A process focus." Research in organizational behavior: 141-197.

Ingraham, P. W. and A. E. Kneedler (2000). Dissecting the black box: Toward a model and measures of government management performance. Washington D.C, Georgetown, University press.

ISOS (2014a). Some issues in the appraisal in the public sector. Hanoi, Vietnam, Institute for State organizational sciences.

ISOS (2014b). Some orientation and solutions for innovation in performance assessment of civil servants in the state administrative agencies. Presented in the conference: Performance assessment of civil servants: International experiences and Vietnam's practices, Institute of state Organization science, Ministry of Home Affairs, Vietnam.

Iurato, J. M. (2007). Leadership trust and member motivation in community mental health organizations, Doctoral Dissertation, Walden University.

Jacobs, C. (2004). "The challenge of public sector reform in EC accession countries: reflections from the Baltics, especially Latvia." Public Administration and Development 24(4): 321-331. 
Jacobs, R., et al. (1980). "Expectations of behaviorally anchored rating scales." Personnel Psychology 33(3): 595-640.

Jankulović, A. and V. Škorić (2013). "Performance management system implementation in a Southeast European transitional country." Journal for East European Management Studies 18(2): 173-190.

Jawahar, I. (2006). "An investigation of potential consequences of satisfaction with appraisal feedback." Journal of Leadership \& Organizational Studies 13(2): 14-28.

Jiang, X. (2010). "How to motivate people working in teams." International Journal of Business and Management 5(10): 223.

Johnson, G. (2010). Research methods for public administrators, NY, Routledge.

Johnson, R. B. and A. J. Onwuegbuzie (2004). "Mixed methods research: A research paradigm whose time has come." Educational researcher 33(7): 14-26.

Johnson, R. B., et al. (2007). "Toward a definition of mixed methods research." Journal of mixed methods research 1(2): 112-133.

Jöreskog, K. G. and D. Sörbom (1982). "Recent developments in structural equation modeling." Journal of marketing research 19(4): 404-416.

Judge, T. A., et al. (2001). "The job satisfaction-job performance relationship: A qualitative and quantitative review." 127(3): 376-407.

Kalgin, A., et al. (2018). "Performance management and job-goal alignment: A conditional process model of turnover intention in the public sector." International Journal of Public Sector Management 31(1): 65-80.

Kamoche, K. (2001). "Human resources in Vietnam: The global challenge." Thunderbird international business review 43(5): 625-650.

Kaufmann, D., et al. (1999). "Governance matters (World Bank Policy Research Working Paper No. 2196)." Washington, DC: World Bank.

Kearney, C., et al. (2009). "Public and private sector entrepreneurship: similarities, differences or a combination?" Journal of Small Business and Enterprise Development 16(1): 26-46.

Kelloway, E. K. (1998). Using LISREL for structural equation modeling: A researcher's guide. Thousand Oaks, CA, Sage.

Ketelaar, A., et al. (2007). "Performance-based arrangements for senior civil servants OECD and other country experiences." OECD Working Papers on Public Governance 2007/5(5): 0_1.

Ketels, C., et al. (2010). Vietnam competitiveness report 2010. Hanoi, Vietnam, Central Institute for Economic Management.

Kim, J. (2016). "Impact of performance appraisal justice on the effectiveness of pay-for-performance systems after civil service reform." Public Personnel Management 45(2): 148-170.

Kim, J. W. and K. Jung (2013). "Does Civic Engagement Resolve Accountability Dilemmas in Local Governance." International Journal of Policy Studies 4(1): 87-104. 
Kim, P. S. (2002). "Strengthening the pay-performance link in government: A case study of Korea." Public Personnel Management 31(4): 447-463.

Kim, S.-W., et al. (1996). "The determinants of career intent among physicians at a US Air Force hospital." Human Relations 49(7): 947-976.

Kim, T. (2014). Performance appraisal: determinants of public employees' acceptance, Doctoral Dissertation, Rutgers University.

King-Kauanui, S., et al. (2006). "Impact of human resource management: SME performance in Vietnam." Journal of developmental entrepreneurship 11(01): 79-95.

Kinicki, A. J., et al. (2013). "Development and validation of the performance management behavior questionnaire." Personnel Psychology 66(1): 1-45.

Kirk, J. and M. L. Miller (1986). Reliability and validity in qualitative research. Beverly Hills, CA, Sage.

Kleinbaum, D. G., et al. (1988). Applied Regression Analysis and Other Multivariate Methods. Boston, PWS-Kent Publishing Company.

Kleingeld, A., et al. (2004). "Participation in the design of performance management systems: a quasiexperimental field study." Journal of Organizational Behavior 25(7): 831-851.

Kline, P. (1992). Handbook of psychological testing. London, Routledge.

Ko, J. and S. Hur (2014). "The impacts of employee benefits, procedural justice, and managerial trustworthiness on work attitudes: Integrated understanding based on social exchange theory." Public Administration Review 74(2): 176-187.

Kong, D., et al. (2013). Individual performance appraisal in the government of Korea. Knowledge Sharing Program: KSP Modularization 2012. Seoul, South Korea, KDI School of Public Policy and Management.

Koonmee, K. (2009). Implementing the performance management system in the Thai public sector. The 2009 International Conference on Human Resource Development (2009 IHRD).

Kotter, J. P. and L. A. Schlesinger (1979). "Choosing strategies for change." Harvard Business Review(March - April): 106-114.

Koubek, J. and C. Brewster (1995). "Human resource management in turbulent times: HRM in the Czech Republic." International Journal of Human Resource Management 6(2): 223-247.

Kozlowski, S. W., et al. (1998). "Games raters play: Politics, strategies, and impression management in performance appraisal." Performance appraisal: State of the art in practice: 163-205.

Kragh, S. U. (2012). "The anthropology of nepotism: Social distance and reciprocity in organizations in developing countries." International Journal of Cross Cultural Management 12(2): 247-265.

Kuvaas, B. (2011). "The interactive role of performance appraisal reactions and regular feedback." Journal of Managerial Psychology 26(2): 123-137.

Lacho, K. J., et al. (1991). "Performance appraisal in local government: A current update." Public Productivity \&amp; Management Review 14(3): 281-296. 
Lah, T. and J. L. Perry (2008). "The diffusion of the Civil Service Reform Act of 1978 in OECD countries: A tale of two paths to reform." Review of Public Personnel Administration 28(3): 282-299.

Larson Jr, J. R. (1989). "The dynamic interplay between employees' feedback-seeking strategies and supervisors' delivery of performance feedback." Academy of management review 14(3): 408-422.

Latham, G. P., et al. (2008). "Goal setting and performance management in the public sector." International Public Management Journal 11(4): 385-403.

Latham, G. P. and E. A. Locke (2007). "New developments in and directions for goal-setting research." European Psychologist 12(4): 290-300.

Latham, G. P. and S. Mann (2006). Advances in the science of performance appraisal: Implications for practice. Chichester, West Sussex, John Wiley \& Sons.

Lavy, V. (2007). "Using performance-based pay to improve the quality of teachers." The future of children(17 (Spring (1) (2007)): 87-109.

Law, D. R. (2007). "Appraising performance appraisals: A critical look at an external control management technique." International Journal of Reality Therapy 26(2): 18.

Law, K. S., et al. (2000). "Effect of supervisor-subordinate guanxi on supervisory decisions in China: An empirical investigation." International Journal of Human Resource Management 11(4): 751-765.

Lawler, E. E. (1994). "Performance management: The next generation." Compensation \& Benefits Review 26(3): 16-19.

Lawler, E. E. (2003). "Reward practices and performance management system effectiveness." Organizational Dynamics 32(4): 396-404.

Lawler, E. E., et al. (2012). "What Makes Performance Appraisals Effective?" Compensation \& Benefits Review 44(4): 191-200.

Lawler, E. E. and M. McDermott (2003). "Current performance management practices-Examining the varying impacts." World at Work Journal 12(2): 49-60.

Le, B. X. (2011). Some issues on the reform of administration appratus and the operation mechanism. Paper presented at the conference on Public administration reform in Vietnam under the lens of academics. Hanoi, Vietnam.

Le, K. (2013). Clarifying 30\% of public officials are unqualified. Tuoitre. Hanoi, Retrieved from: http://tuoitre.vn.

Le, S. (2015). At the end of 2015, poor households is only 4.5 percent. Government newspaper, Retrieved from: http://baochinhphu.vn.

Le, T. S. (2011). Seeking a correct evaluation of the PAR program after 10 years. Paper presented at the conference on Public administration reform in Vietnam under the lens of academics. Hanoi, Vietnam.

Leavitt, W. M. and G. Johnson (1998). "Professional notes: Employee discipline and the postbureaucratic public organization: A challenge in the change process." Review of Public Personnel Administration 18(2): 73-81. 
Lebas, M. J. (1995). "Performance measurement and performance management." International journal of production economics 41(1-3): 23-35.

Lee, H.-W. (2017). "Performance-based human resource management and federal employee's motivation: moderating roles of goal-clarifying intervention, appraisal fairness, and feedback satisfaction." Review of Public Personnel Administration: 1-26.

Leedy, P. D. and J. E. Ormrod (2005). Practical research: planning and desing (9th ed). Upper Saddle River, New Jersey, Pearson Education.

Legge, K. (1978). Power, innovation, and problem-solving in personnel management. London, MacGraw-Hill Book Company.

Lerner, J. S. and P. E. Tetlock (1999). "Accounting for the effects of accountability." Psychological bulletin 125(2): 255.

Lewis, L. K. (1999). "Disseminating information and soliciting input during planned organizational change: Implementers' targets, sources, and channels for communicating." Management Communication Quarterly 13(1): 43-75.

Lewis, L. K. (2006). "Employee perspectives on implementation communication as predictors of perceptions of success and resistance." Western Journal of Communication 70(1): 23-46.

Lincoln, Y. S. and E. G. Guba (1985). Naturalistic inquiry. Beverly Hills, CA, Sage.

Lindholm, N. (1999). "National culture and performance management in MNC subsidiaries." International studies of management \& organization 29(4): 45-66.

Linz, J. J. and A. Stepan (1996). Problems of democratic transition and consolidation: Southern Europe, South America, and post-communist Europe, Johns Hopkins University.

Liu, X. and K. Dong (2012). "Development of the Civil Servants Performance Appraisal System in China: Challenge and Improvement." Review of Public Personnel Administration 32(2): 149-168.

Locke, E. A. (1968). "Toward a theory of task motivation and incentives." Organizational behavior and human performance $3(2)$ : 157-189.

Locke, E. A. (1976). The nature and causes of job satisfaction. Chicago Rand McNally, .

Locke, E. A. and G. P. Latham (2002). "Building a practically useful theory of goal setting and task motivation: A 35-year odyssey." American psychologist 57(9): 705.

Longenecker, C. O. (1997). "Why managerial performance appraisals are ineffective: causes and lessons." Career Development International 2(5): 212-218.

Longenecker, C. O. and D. A. Gioia (2001). Confronting the" politics" in performance appraisal. Business Forum, California State University, Los Angeles, School of Business and Economics.

Longenecker, C. O. and S. J. Goff (1992). "Performance appraisal effectiveness: A matter of perspective." SAM Advanced Management Journal 57(2): 17-17.

Lu, C. (2006). "Assessing construct validity: The utility of factor analysis." Journal of Educational Measurement and Statistics 15(2): 79-94. 
Luecke, R. and B. J. Hall (2006). Performance management: Measure and improve the effectiveness of your employees, Harvard Business Press.

Luo, Y. (1997). "Guanxi: Principles, philosophies, and implications." Human systems management 16(1): 43.

Ma, L., et al. (2015). "Public Employees' Perceived Promotion Channels in Local China: Merit-based or Guanxi-orientated?" Australian Journal of Public Administration 74(3): 283-297.

MacCallum, R. C., et al. (1996). "Power analysis and determination of sample size for covariance structure modeling." Psychological methods 1(2): 130.

MacLeod, D. and N. Clarke (2011). Engaging for success: enhancing performance through employee engagement, a report to Government. London, Department for Business, Innovation and Skills.

Maczyński, J., et al. (1994). "Culture and leadership styles: A comparison of Polish, Austrian, and US managers." Polish Psychological Bulletin.

Mafini, C., et al. (2014). "Factors Contributing To The Unsuccessful Implementation Of The BSC In A South African Provincial Treasury Department." The International Business \& Economics Research Journal (Online) 13(6): 1539.

Magee, K. C. (2002). The impact of organizational culture on the implementation of performance management, Doctoral Dissertation, Georgia State University.

Mahler, J. (1997). "Influences of organizational culture on learning in public agencies." Journal of Public Administration Research and Theory 7(4): 519-540.

Mai, P. (2016). 225,000 Bachelor's and Master's degree graduates are unemployed. Vietnamnet, Retrieved from: http://english.vietnamnet.vn.

Malterud, K. (2001). "Qualitative research: standards, challenges, and guidelines." The lancet 358(9280): 483-488.

Manasa, K. and N. Reddy (2009). "Role of training in improving performance." The IUP Journal of Soft Skills 3(4): 72-80.

Mani, B. G. (2002). "Performance appraisal systems, productivity, and motivation: A case study." Public Personnel Management 31(2): 141-159.

Manoharan, T., et al. (2009). "Employee Performance Appraisal Using Data Envelopment Analysis: A Case Study." Research \& Practice in Human Resource Management 17(1): 92-111.

MARD (2013). Performance appraisal in Ministry of Agriculture and Rural Development. Reforming performance appraisal in administration agencies. Institute of State Organization Science, Ministry of Agriculture and Rural Development, Hanoi, Vietnam.

Markus, L. H. and L. Markus (2004). "Performance Management-Problems and Potential." Singapore: Centranum LTD.

Marshall, C. and G. B. Rossman (2006). Designing qualitative research, Thousand Oaks, CA, Sage publications. 
Marshall, N. (1998). "Pay-for-performance systems: Experiences in Australia." Public Productivity \& Management Review: 403-418.

Marshall, V. and R. E. Wood (2000). "The dynamics of effective performance appraisal: an integrated model." Asia Pacific Journal of Human Resources 38(3): 62-90.

Maslowski, R., et al. (2007). "The effect of school autonomy and school internal decentralization on students' reading literacy." School Effectiveness and School Improvement 18(3): 303-334.

Mau, D. V., et al. (2012). State administration and administration technique. Ha Noi, Science and Technology.

Maxwell, J. A. (2005). Qualitative research design: An interactive approach, Sage publications.

Mayer, R. C. and J. H. Davis (1999). "The effect of the performance appraisal system on trust for management: A field quasi-experiment." Journal of Applied Psychology 84(1): 123.

McAdam, R. and F. Mcgeough (2000). "Implementing total productive maintenance in multi-union manufacturing organizations: overcoming job demarcation." Total Quality Management 11(2): 187-197.

McClelland, D. C. (1973). "Testing for competence rather than for" intelligence."." American psychologist 28(1): 1 .

McCourt, W. and A. Ramgutty-Wong (2003). "Limits to strategic HRM: the case of the Mauritian civil service." International Journal of Human Resource Management 14(4): 600-618.

McCracken, M. J., et al. (2001). "Measuring organizational performance in the hospital industry: an exploratory comparison of objective and subjective methods." Health services management research 14(4): 211-219.

McShane, S. and A. Travaglione (2010). Organisational behaviour on the Pacific Rim, McGraw-Hill Higher Education.

Mendonca, M. and R. N. Kanungo (1996). "Impact of culture on performance management in developing countries." International Journal of Manpower 17(4/5): 65-75.

Meng, F. and J. Wu (2015). "Merit pay fairness, leader-member exchange, and job engagement: Evidence from Mainland China." Review of Public Personnel Administration 35(1): 47-69.

Mero, N. P., et al. (2007). "Accountability in a performance appraisal context: The effect of audience and form of accounting on rater response and behavior." Journal of management 33(2): 223-252.

Merritt, D. (2007). "Appraising the performance appraisal." Supervision 68(4): 3-5.

Meyer-Sahling, J.-H. (2009). "Varieties of legacies: a critical review of legacy explanations of public administration reform in East Central Europe." International Review of Administrative Sciences 75(3): 509-528.

Meyer-Sahling, J.-H. (2012). "Civil Service Professionalisation in the Western Balkans." Sigma Papers (48): 0_1.

Meyer, J. P., et al. (1997). Commitment in the workplace. Thousand Oaks, CA, Sage Publications. 
Meyer, J. P., et al. (1993). "Commitment to organizations and occupations: Extension and test of a threecomponent conceptualization." Journal of Applied Psychology 78(4): 538.

Milikić, B. B., et al. (2012). "Two decades of post-socialism in Serbia: Lessons learned and emerging issues in human resource management." Journal for East European Management Studies 17(4): 445463.

Miller, D. (2001). "Successful change leaders: what makes them? What do they do that is different?" Journal of Change Management 2(4): 359-368.

Miller, V. D., et al. (1994). "Antecedents to willingness to participate in a planned organizational change." Journal of Applied Communication Research 22(1): 59-80.

Milliman, J., et al. (1998). "The impact of national culture on HRM practices: The case of performance appraisal." Advances in international comparative management 12: 157-183.

Mitchell, T. R. and C. O'Reilly (1983). "Managing poor performance and productivity in organizations." Research in personnel and human resources management 1: 201-234.

Mizrahi, S. and Y. Minchuk (2017). "Performance management in a decentralized setting: Monitoring and gaming in the financial services industry." Managerial and Decision Economics 38(6): 742-753.

MOH (2018). Self-financing mechanism in public health institutions. Review of Finance, Retrieved from: www.tapchitaichinh.vn.

MOHA (2011). The review report of the PAR Master programme (2001 - 2010). Ha Noi, Vietnamese Ministry of Home Affairs.

MOHA (2014a). Some regulations on the evaluation of civil servants of the Vietnam government. Hanoi, Vietnam, Vietnamese Ministry of Home Affairs.

MOHA (2014b). Appraising and rating civil servants and public employees in 2014. Hanoi, Vietnam, Vietnamese Ministry of Home Affairs.

MOJ (2016). Evaluating the curent situation of institution reform. Hanoi, Vietnam, Vietnamese Ministry of Justice.

Montoya, S. and J. D. Graham (2008). Modernizing the Federal Government: Paying for Performance. Santa Monica, CA, Rand Corporation.

Moon, M. J. and C. Hwang (2013). "The state of civil service systems in the Asia-Pacific region: a comparative perspective." Review of Public Personnel Administration 33(2): 121-139.

Moriarty, P. and D. Kennedy (2002). "Performance measurement in public sector services: problems and potential." Performance Measurement and Management: Research and Action, Cranfield School of Management, UK.

Mowday, R. T., et al. (1982). "Organizational linkage: the psychology of commitment, absenteeism and turnover." Organizational and Occupational Psychology 10(3): 2008.

Mowday, R. T., et al. (1979). "The measurement of organizational commitment." Journal of vocational behavior 14(2): 224-247. 
Moynihan, D. P. and S. K. Pandey (2004). "Testing how management matters in an era of government by performance management." Journal of Public Administration Research and Theory 15(3): 421-439.

Munene, J. C., et al. (2000). "Development in Sub-Saharan Africa: cultural influences and managers' decision behaviour." Public Administration \& Development 20(4): 339.

Murphy, G. D. and G. Southey (2003). "High performance work practices: perceived determinants of adoption and the role of the HR practitioner." Personnel Review 32(1): 73-92.

Murphy, K. R. and W. K. Balzer (1989). "Rater errors and rating accuracy." Journal of Applied Psychology 74(4): 619.

Murphy, K. R. and J. Cleveland (1995). Understanding performance appraisal: Social, organizational, and goal-based perspectives. Thousand Oaks, CA, Sage.

Murphy, K. R., et al. (1984). "Effects of the purpose of rating on accuracy in observing teacher behavior and evaluating teaching performance." Journal of Educational Psychology 76(1): 45.

Mustapha, M. and N. Daud (2012). "Perceived performance appraisal effectiveness, career commitment and turnover intention of knowledge workers." International Journal of Business and Social Science 3(19).

Mwita, J. I. (2003). The Role of Motivation in Performance Management: The case of performancerelated-pay schemes in British local authorities, University of Birmingham.

Nadler, D. A. (1997). Champions of change: How CEOs and their companies are mastering the skills of radical change. San Francisco, Jossey-Bass.

Nambi Karuhanga, B. and A. Werner (2013). "Challenges impacting performance management implementation in public universities: A case of Uganda." African Journal of Economic and Management Studies 4(2): 223-243.

Nankervis, A. R. and R.-L. Compton (2006). "Performance management: theory in practice?" Asia Pacific Journal of Human Resources 44(1): 83-101.

Narayanan, A. (2012). "A review of eight software packages for structural equation modeling." The American Statistician 66(2): 129-138.

Nelissen, P. and M. van Selm (2008). "Surviving organizational change: How management communication helps balance mixed feelings." Corporate Communications: An International Journal 13(3): 306-318.

Ngo, C. T. (2011). Reforming the training practice and improving the quality of HR in the public sector. Hanoi, Vietnam, National Academy of public administration.

Ngo, C. T. (2016). Quality of public service: the core issue of the public administration. Hanoi, Vietnam, National steering committee of PAR.

Ngo, H.-y., et al. (2014). "Linking HRM competency to firm performance: an empirical investigation of Chinese firms." Personnel Review 43(6): 898-914.

Ngo, P. H. (2011). Reforming institution in Vietnam through the lens of scheme 30. Paper presented at the conference on Public administration reform in Vietnam under the lens of academics. Hanoi, Vietnam. 
Ngoc, D. (2011). Where does inflation in Vietnam come from? VNeconomy. Vietnam, Retrieved from: http://vneconomy.vn.

Ngoc, T. (2016). 17,000 billion is paying for incompetent civil servants. Vnexpress.net, Retrieved from: https://kinhdoanh.vnexpress.net.

Ngok, K. and G. Zhu (2007). "Marketization, globalization and administrative reform in China: A zigzag road to a promising future." International Review of Administrative Sciences 73(2): 217-233.

Nguoilaodong (2017). Low salary, public employees resign. Nguoi lao dong, Retrieved from: https://nld.com.vn.

Nguyen, A. K. (2014). Reforming appraisal practice in the State Bank of Vietnam. Paper presented at the conference on reforming appraisal practice in the State Bank of Vietnam.

Nguyen, C. T. (2015). Irony of public employee appraisal. Vietnam.net, Retrieved from: http://vietnamnet.vn/vn/.

Nguyen, D. T. U. (2017). In Vietnam, the best education can lead to worse job perspects. Bloomberg, Retrieved from: https://www.bloomberg.com.

Nguyen, H. C. (2017). Vietnam's competitiveness: a 10 year review. The Saigon Times, Retrieved from: www.thesaigontimes.vn.

Nguyen, H. K. (2017). Continue enhancing the quality of the cadre practice according to Resolution No 4 of the 12th Central Committee of the Communist Party Communist Magazine.

Nguyen, H. T. H. (2011). Some issues of capacity building in conducting public service to meet PAR demands. Paper presented at the conference on Public administration reform in Vietnam through the lens of academics. Hanoi, Vietnam.

Nguyen, T. D. (2011). Reforming the recruitment and selection practices in the context of PAR. Paper presented at the conference on Public administration reform in Vietnam through the len of academics. Hanoi, Vietnam, National Accademy of Public Administration.

Nham, T. C. (2013). Developing the contingent of cadres according to Ho Chi Minh's thoughts. Party Development Magazine. Retrieved from: http://xaydungdang.org.vn.

Nho, T. (2018). Study tour for tourist purpose by state budget is corruption. Voice of Vietnam, Retrieved from: http://vov.vn.

Norman, R. (2013). Managing for outcomes new challenges for New Zealand public service chief executives. Public management research. Madision, Wisconsin, USA.

Nyhan, R. C. (2000). "Changing the paradigm: Trust and its role in public sector organizations." The American Review of Public Administration 30(1): 87-109.

O'Boyle, I. and P. Cummins (2013). "Examining theories of individual performance management." Training \& Management Development Methods 27(5): 369-377.

O'Leary, Z. (2013). The essential guide to doing your research project, Thousand Oaks, CA, Sage. 
O'Reilly III, C. A. and B. A. Weitz (1980). "Managing marginal employees: The use of warnings and dismissals." Administrative science quarterly 25(3): 467-484.

O'Leary, Z. (2004). The essential guide to doing research, Thousand Oaks, CA, Sage.

Obong'o, S. O. (2009). "Implementation of performance contracting in Kenya." International Public Management Review 10(2): 66-84.

Ochoti, G. N., et al. (2012). "Factors influencing employee performance appraisal system: a case of the ministry of state for provincial administration \& internal security, Kenya." International Journal of Business and Social Sciences 3(20): 37-41.

Oh, S. S. and G. B. Lewis (2009). "Can performance appraisal systems inspire intrinsically motivated employees?" Review of Public Personnel Administration 29(2): 158-167.

Ohemeng, F. L., et al. (2018). "The impact of employee perception on the successful institutionalisation and implementation of performance management systems in developing countries: The perspective from Ghana's public service." Public Administration and Development 38(2): 75-86.

Ohemeng, F. L., et al. (2015). "Performance Appraisal and Its Use for Individual and Organisational Improvement in the Civil Service of Ghana: The Case of Much Ado about Nothing?" Public Administration and Development 35(3): 179-191.

Ohemeng, F. L. K. (2009). "Constraints in the Implementation of Performance Management Systems in Developing Countries The Ghanaian Case." International Journal of Cross Cultural Management 9(1): 109-132.

Ohemeng, F. L. K., et al. (2018). "Giving sense and changing perceptions in the implementation of the performance management system in public sector organisations in developing countries." International Journal of Public Sector Management 31(3): 372-392.

OPM, U. S. (2017). A handbook for measuring employee performance, Office of Personnel Management, US.

Osborne, D. and P. Plastrik (1997). Banishing Bureaucracy: The Five Strategies for Reinventing Government. 1 Jacob Way, Reading, MA 01867, Addison-Wesley Publishing Company.

Osigweh, C. A., et al. (1993). "Conceptions of employee responsibilities and rights in the United States and the People's Republic of China." International Journal of Human Resource Management 4(1): 85112.

Otley, D. (1999). "Performance management: a framework for management control systems research." Management Accounting Research 10(4): 363-382.

Ouyang, Y. (2011). "An exploration of LMX and personal guanxi on job performance: The mediating effects of CSR." Journal of Global Business Issues 5(1): 1.

Painter, M. (2003). "Public administration reform in Vietnam: problems and prospects." Public Administration and Development 23(3): 259-271.

Parham, C. P. J. (2003). The Construct of Substance Abuse Enabling Applied to Poor Performance Management: How Managers Deal With Poor Performing Employees, Doctoral Dissertation, Virginia Polytechnic Institute. 
Park, S. (2014). "Motivation of Public Managers as Raters in Performance Appraisal Developing a Model of Rater Motivation." Public Personnel Management 43(4): 387-414.

Parzefall, M.-R. and D. M. Salin (2010). "Perceptions of and reactions to workplace bullying: A social exchange perspective." Human Relations 63(6): 761-780.

Patten, T. H. (1977). Pay: employee compensation and incentive plans. New York, The Free Press.

Patton, M. (2002). Qualitative Research and Evaluation Methods, 209-339. Thousand Oaks, CA, Sage.

Perry, J. L., et al. (2009). "Back to the future? Performance-related pay, empirical research, and the perils of persistence." Public Administration Review 69(1): 39-51.

Petrescu, M. (2013). "Marketing research using single-item indicators in structural equation models." Journal of Marketing Analytics 1(2): 99-117.

Pettijohn, C., et al. (2001). "Are performance appraisals a bureaucratic exercise or can they be used to enhance sales-force satisfaction and commitment?" Psychology \& Marketing 18(4): 337-364.

Pfeffer, J. (1998). "Six dangerous myths about pay." Harvard Business Review 76: 108-120.

Pham, H. N. (2018). Leadership and Public Sector Reform in Vietnam. Leadership and Public Sector Reform in Asia, Emerald Publishing Limited: 127-149.

Pham, T. Q. (2008). Why competent public officials leave public organizations. DanTri, Retrieved from: www.dantri.com.vn.

Phan, N. (2014). Faculty perceptions of the performance appraisal process, California State University, Fullerton.

Phuong, M. (2012). Bribery for positions and power. Tamnhin, Retrieved from: www.tamnhin.net.

Pichler, S. (2012). "The social context of performance appraisal and appraisal reactions: A metaanalysis." Human Resource Management 51(5): 709-732.

Pinder, C. C. (2014). Work motivation in organizational behavior. New York, Psychology Press.

Plimmer, G., et al. (2017). "Opening the black box: The mediating roles of organisational systems and ambidexterity in the HRM-performance link in public sector organisations." Personnel Review 46(7): $1434-1451$.

Podsakoff, P. M. and D. W. Organ (1986). "Self-reports in organizational research: Problems and prospects." Journal of management 12(4): 531-544.

Pollitt, C. (2005). "Performance management in practice: A comparative study of executive agencies." Journal of Public Administration Research and Theory 16(1): 25-44.

Poocharoen, O.-o. and A. Brillantes (2013). "Meritocracy in Asia Pacific: Status, issues, and challenges." Review of Public Personnel Administration 33(2): 140-163.

Poon, J. M. (2004). "Effects of performance appraisal politics on job satisfaction and turnover intention." Personnel Review 33(3): 322-334. 
Poon, Y., et al. (2009). The Reform of the Civil Service System as Vietnam moves into the MiddleIncome Country Category. UNDP Vietnam, Hanoi, UNDP: 199.

Poor, J. and G. Plesoianu (2010). "Human resource management under change in the Romanian civil service in an international context." Employee Relations 32(3): 281-309.

Pooyan, A. and B. J. Eberhardt (1989). "Correlates of performance appraisal satisfaction among supervisory and nonsupervisory employees." Journal of Business Research 19(3): 215-226.

Porter, D. (2004). Review of governance and public management for Sri Lanka, Manila: Asian Development Bank.

Porter, L. W., et al. (1974). "Organizational commitment, job satisfaction, and turnover among psychiatric technicians." Journal of Applied Psychology 59(5): 603.

Posthuma, R. A. and M. A. Campion (2008). "Twenty Best Practices for Just Employee Performance Reviews." Compensation \& Benefits Review 40(1): 47-55.

Pulakos, E., et al. (2012). "Building a high-performance culture: A fresh look at performance management." Effective Practices GuidelinesAlexandria, VA: SHRM Foundation.

Pulakos, E. D. (2009). Performance management: A new approach for driving business results, Chichester, West Susex, John Wiley \& Sons.

Pulakos, E. D. and R. s. O'Leary (2011). "Why is performance management broken?" Industrial and Organizational Psychology 4(2): 146-164.

Punch, K. F. (2013). Introduction to social research: Quantitative and qualitative approaches. London, Sage.

Rainey, H. G., et al. (1976). "Comparing public and private organizations." Public Administration Review 36(2): 233-244.

Randall, D. M. (1990). "The consequences of organizational commitment: Methodological investigation." Journal of Organizational Behavior 11(5): 361-378.

Randma-Liiv, T. (2005). "Performance management in transitional administration: introduction of payfor-performance in the Estonian civil service." Journal of Comparative Policy Analysis: Research and Practice 7(1): 95-115.

Rao, T. V. (2004). Performance Management and Appraisal Systems: HR tools for global competitiveness, SAGE Publications India.

Redman, T., et al. (2000). "Performance appraisal in an NHS hospital." Human Resource Management Journal 10(1): 48-62.

Reinke, S. J. (2003). "Does the form really matter? Leadership, trust, and acceptance of the performance appraisal process." Review of Public Personnel Administration 23(1): 23-37.

Renko, M., et al. (2015). "Understanding and measuring entrepreneurial leadership style." Journal of Small Business Management 53(1): 54-74.

Rhoades, L., et al. (2001). "Affective commitment to the organization: the contribution of perceived organizational support." Journal of Applied Psychology 86(5): 825. 
Richard, J., et al. (2015). "Publishing success of marketing academics: antecedents and outcomes." European Journal of Marketing 49(1/2): 123-145.

Risher, H. (2002). "Pay-for-performance: The keys to making it work." Public Personnel Management 31(3): 317-332.

Risher, H. (2011). "Getting performance management on track." Compensation \& Benefits Review 43(5): 273-281.

Roberts, G. E. (2003). "Employee performance appraisal system participation: A technique that works." Public Personnel Management 32(1): 89-98.

Roberts, G. E. and T. Pavlak (1996). "Municipal Government Personnel Professionals and Performance Appraisal: Is There a Consensus on the Characteristics of an Effective Appraisal System?" Public Personnel Management 25(3): 379-408.

Roberts, G. E. and T. Reed (1996). "Performance Appraisal Participation, Goal Setting and Feedback The Influence of Supervisory Style." Review of Public Personnel Administration 16(4): 29-60.

Roberts, P., et al. (2006). "Reliability and validity in research." Nursing standard 20(44): 41-45.

Robertson, L. and B. Hale (2011). "Interviewing older people; relationships in qualitative research." Internet Journal of Allied Health Sciences and Practice 9(3): 1-8.

Rodgers, R. and J. E. Hunter (1991). "Impact of management by objectives on organizational productivity." Journal of Applied Psychology 76(2): 322.

Rossman, G. B. and S. F. Rallis (2003). Learning in the field: An introduction to qualitative research. Thousand Oaks, CA, Sage.

Rowshan, A. G., et al. (2015). "Investigation of Nepotism in Public Sector Organizations in Iran and its Impact on Employee's Performance." International Journal of Management, Accounting \& Economics 2: $189-199$.

Rubel, M. R. B. and D. M. H. Kee (2015). "Perceived Fairness of Performance Appraisal, Promotion Opportunity and Nurses Turnover Intention: The Role of Organizational Commitment." Asian Social Science 11(9): 183-197.

Ryu, S. H. (2010). Performance management in Korean government, Doctoral Dissertation, Arizona State University.

Saad, S. K. (2014). "Investigating the Effectiveness of the Performance Appraisal Process in the Egyptian Tourism Companies." Journal of Association of Arab Universities for Tourism and Hospitality 11(2): 111-121.

Saldaña, J. (2009). An introduction to codes and coding: the coding manual for qualitative researchers. London, Sage.

Samaratunge, R., et al. (2008). "Public sector reforms and accountability: The case of south and Southeast Asia." Public management review 10(1): 101-126.

Sanwong, K. (2008). "The development of a 360-degree performance appraisal system: a university case study." International Journal of Management 25(1): 16. 
Schein, E. H. (1985). Organisational culture and leadership: A dynamic view. San Francisco, CA, Jossey-Bass.

Schick, A. (1998). "Why most developing countries should not try New Zealand's reforms." The World Bank Research Observer 13(1): 123-131.

Schleicher, D. J., et al. (2018). "Putting the System Into Performance Management Systems: A Review and Agenda for Performance Management Research." Journal of management 44(6): 2209-2245.

Schmit, M. J. and S. P. Allscheid (1995). "Employee attitudes and customer satisfaction: Making theoretical and empirical connections." Personnel Psychology 48(3): 521-536.

Schniederjans, M. J. and P. A. Stoeberl (1986). "Coping Strategies for Managing the Ineffective Subordinate in Production/Operations Management." Management Research News 9(4): 10-15.

Schwartz, A. E. (1995). "Counseling the marginal performer." The CPA Journal 65(2): 66.

Scoppa, V. (2009). "Intergenerational transfers of public sector jobs: a shred of evidence on nepotism." Public Choice 141(1): 167-188.

Selmer, J. and R. Chiu (2004). "Required human resources competencies in the future: a framework for developing HR executives in Hong Kong." Journal of World Business 39(4): 324-336.

Seniwoliba, J. A. (2014). "Assessing the performance appraisal concept of the Local Government Service in Ghana." African Journal of Business Management 8(15): 599-611.

Shafie, H. b. (1996). "Malaysia's experience in implementing the new performance appraisal system." Public Administration and Development 16(4): 341-352.

Shahina, J. and S. D. Sumod (2015). "It's time to bring performance appraisal into the twenty-first century: The lessons from companies like Cisco, Google and Infosys." Human Resource Management International Digest 23(7): 23-26.

Shields, J. (2007). Managing employee performance and reward: concepts, practices, strategies. Sydney, Cambridge University Press.

Shin, D., et al. (1998). "Critical implementation issues in total quality management." SAM Advanced Management Journal 63(1): 10.

Siaguru, F. (2011). "Performance appraisal systems: Procedural and implementation issues in Papua New Guinea." Journal of Management Policy and Practice 12(5): 116.

Siddiquee, N. A. (2003). "Human resource management in Bangladesh Civil Service: constraints and contradictions." International Journal of Public Administration 26(1): 35-60.

Silva, E. D. and D. Wright (2008). "Researching cultural capital: Complexities in mixing methods." Methodological Innovations Online 2(3): 50-62.

Skinner, B. F. (1953). Science and human behavior. New York, Macmillan.

Smith, B. (2012). The effects of accountability on leniency reduction in self-and peer ratings on teambased performance appraisals, Doctoral Dissertation, Clemson University. 
Somerick, N. M. (1993). "Strategies for improving employee relations by using performance appraisals more effectively." Public Relations Quarterly 38(3): 37.

Spector, P. E. (1986). "Perceived control by employees: A meta-analysis of studies concerning autonomy and participation at work." Human Relations 39(11): 1005-1016.

Spector, P. E. (1994). "Job satisfaction survey." Tampa, Florida: Department of Psychology, University of South Florida.

Spector, P. E. (2008). Industrial and organizational psychology: research and practice. New York, John Wiley.

Stanton, P. and A. Nankervis (2011). "Linking strategic HRM, performance management and organizational effectiveness: perceptions of managers in Singapore." Asia Pacific Business Review 17(01): 67-84.

Stanton, P. and H. T. Pham (2014). "Managing employee performance in an emerging economy: perceptions of Vietnamese managers." Asia Pacific Business Review 20(2): 269-285.

Stazyk, E. C. (2009). Crowding out intrinsic motivation? The role of performance-related pay. 10th National Public Management Research Conference.

Steers, R. M. (1977). "Antecedents and outcomes of organizational commitment." Administrative science quarterly 22(1): 46-56.

Stoeberi, P. and M. J. Schniederjans (1981). "The ineffective subordinate: a management survey." The Personnel administrator 26(2): 72-76.

Stoskopf, G. A. (2002). "Taking performance management to the next level." Workspan 45(2): 28-30.

Suhomlinova, O. (2006). "Toward a Model of Organizational Co-Evolution in Transition Economies." Journal of Management Studies 43(7): 1537-1558.

Suutari, V. and K. Riusala (2001). "Leadership styles in central eastern Europe: Experiences of Finnish expatriates in the Czech Republic, Hungary and Poland." Scandinavian Journal of Management 17(2): 249-280.

Taylor, H. (1992). "Public sector personnel management in three African countries: current problems and possibilities." Public Administration and Development 12(2): 193-207.

Taylor, J. (2007). "The impact of public service motives on work outcomes in Australia: A comparative multi-dimensional analysis." Public administration 85(4): 931-959.

Taylor, J. and L. Beh (2013). "The impact of pay-for-performance schemes on the performance of Australian and Malaysian government employees." Public management review 15(8): 1090-1115.

Taylor, P. and M. O'Driscoll (1993). "Functions and implementation of performance appraisal systems in New Zealand organizations." Asia Pacific Journal of Human Resources 31(1): 20-32.

Taylor, P. J. and J. L. Pierce (1999). "Effects of introducing a performance management system on employees' subsequent attitudes and effort." Public Personnel Management 28(3): 423-452.

Taylor, S. J., et al. (2015). Introduction to qualitative research methods: A guidebook and resource, Hoboken, New Jersey, John Wiley \& Sons. 
Teclemichael Tessema, M. and J. L. Soeters (2006). "Challenges and prospects of HRM in developing countries: testing the HRM-performance link in the Eritrean civil service." The International Journal of Human Resource Management 17(1): 86-105.

Terpstra, D. E., et al. (1996). "A model of human resource information, practice choice and organizational outcomes." Human Resource Management Review 6(1): 25-46.

Thang, L. C. and T. Quang (2005). "Antecedents and consequences of dimensions of human resource management practices in Vietnam." The International Journal of Human Resource Management 16(10): 1830-1846.

Thanh, V. V. (2015). Downsizing at least 10 percent of public employees in central ministries and provincies. Tuoitre, Retrieved from: http://tuoitre.vn.

Thomas, P. G. (2007). "Why is performance-based accountability so popular in theory and difficult in practice." World Summit on Public Governance: Improving the Performance of the Public Sector. Taipei: $1-3$.

Thomas, S. L. and R. D. Bretz Jr (1994). "Research and practice in performance appraisal: Evaluating employee performance in America's largest companies." SAM Advanced Management Journal 59(2): 28.

Thornberry, N. (2006). Lead like an entrepreneur, Blacklick, OH, McGraw Hill Professional.

Thu, H. (2016). Civil servants' salary only meets 50 percent of basic need. Thanhnien, Retrieved from: https://thanhnien.vn.

Thu, H. (2018). Many provincial leaders have backyard enterprises. Vietnam.net, Retrieved from: http://vietnam.net.

Thuy, C. (2011). Why I leave the public sector. Vietnamnet, Retrieved from: http://vietnamnet.vn.

Ting, Y. (1997). "Determinants of job satisfaction of federal government employees." Public Personnel Management 26(3): 313-334.

Tong, C. H., et al. (1999). "Civil service reform in the People's Republic of China: case studies of early implementation." Public Administration \& Development 19(2): 193.

Trang, T. T. (2011). Renovating the training practice for pubic employees in the new context. Paper presented at the conference on public administration reform in Vietnam through the lens of academics. Hanoi, Vietnam.

Trochim, W. M. and J. P. Donnelly (2001). Research methods knowledge base. Cincinnati, OH, Atomic Dog Publishing.

Trung, A. (2014). All are rated as good. E-newspaper of the Communist party of Vietnam, Retrieved from: http://cpv.org.vn.

Tung, A., et al. (2011). "Factors influencing the effectiveness of performance measurement systems." International journal of operations \& production management 31(12): 1287-1310.

Tung, C. (2013). 30 percent of public servants only take an umbrella to their office then take it back. Laodong. Hanoi, Vietnam, Retrieved from: http://laodong.com.vn. 
Tuyen, V. V. (2013). The history of Vietnam public administration. Ha Noi, Science and Technology.

Tuytens, M. and G. Devos (2012). "Importance of system and leadership in performance appraisal." Personnel Review 41(6): 756-776.

Ulrich, D., et al. (1995). "Human resource competencies: An empirical assessment." $\underline{\text { Human Resource }}$ Management 34(4): 473-495.

UNDP (2013). Poverty reduction in Vietnam. UNDP Vietnam, Retrieved from: www.vn.undp.org/.../vietnam/.../Factsheet poverty.

UNDP (2017). "UNDP's evaluation of Vietnam's economic reform." Retrieved 07/07/2018, 2018, from http://www.vn.undp.org/content/vietnam/vi/home/presscenter/undp-in-the-news/_anh-gia-c_a-lhq-undp-v-ci-cach-kinh-t-ca-vit-nam--trin-vng-lhq.html.

US.OPM (2002). Poor performance, Office of Personnel Managementm, US.

Vallance, S. (1999). "Performance appraisal in Singapore, Thailand and the Philippines: A cultural perspective." Australian Journal of Public Administration 58(4): 78-95.

Van Buren, H. and C. R. Leana (2000). Building relational wealth through employment practices. New York, Oxford University Press.

Van Herpen, M., et al. (2005). "The effects of performance measurement and compensation on motivation: An empirical study." De Economist 153(3): 303-329.

Van Muijen, J. and P. Koopman (1994). "The influence of national culture on organizational culture: A comparative study between 10 countries." European Journal of Work and Organizational Psychology 4(4): 367-380.

Verbeeten, F. H. (2008). "Performance management practices in public sector organizations: Impact on performance." Accounting, Auditing \& Accountability Journal 21(3): 427-454.

Verheijen, T. and Y. Dobrolyubova (2007). "Performance management in the Baltic States and Russia: success against the odds?" International Review of Administrative Sciences 73(2): 205-215.

Veselinović, P. (2014). "The reform of the public sector as the key determinant of the transition economy of the Republic of Serbia." Ekonomski horizonti 16(2): 137-155.

Vian, T., et al. (2012). "Confronting corruption in the health sector in Vietnam: patterns and prospects." Public Administration and Development 32(1): 49-63.

Vietnam (2001). Decision no. 136/2001/QD-TTg of September 17, 2001 approving the overall program on state administrative reform, the 2001-2010 period. Government of Vietnam.

Vietnam (2002). The Government's Decree No. 10/2002/ND-CP of January 16, 2002, providing for the financial regime applicable to public-service units with revenues. Government of Vietnam.

Vietnam (2005). The Government's Decree No. 130/2005/ND-CP of October 17, 2005, providing for the regime of autonomy and self-responsibility for use of administrative management payrolls and funds by state agencies. Government of Vietnam. 
Vietnam (2011). Resolution No 30c/NQ-CP dated November 8, 2011 on promulgating the master program on state administration reform in the 2011-2020 period, Government of Vietnam.

Vietnam (2012). Speeding up the reform of public service and public servants. Hanoi, Vietnam, Government of Vietnam

Vietnam (2015). Decree No. 56/2015/ND-CP on the criteria for evaluation and classification of cadres, civil servants and public employees. Government of Vietnam.

Vietnam (2016). Preliminary evaluation of the PAR program of the 2011 - 2020 period. Hanoi, Vietnam, Government of Vietnam.

Vo, H. (2015). Thirty percent of public officials are unqualified accordingto public opinion. Vnexpress, Retrieved from: http://vnexpress.net.

Vo, H. Q. and T. H. Cao (2010). "Factors influencing turnover intention of public officials " Science and technology develoment 13(1Q): 5-16.

Vroom, V. H. (1964). Work and motivation. New York, Wiley.

Vu, A. (2017). Cumbersome administration consumes a large amount of state budget. Thanhnien, Retrieved from: https://thanhnien.vn.

Vu, L. (2014). Over 99\% of public servants are deligent: 'Right, but no body believes that'. Datviet, Retrieved from: http://baodatviet.vn.

Vu, Q. (2011). Public employee salary: how much is enough. VNeconomy, Retrieved from: http://vneconomy.vn.

Vveinhardt, J. and L. Petrauskaite (2013). "Intensity of nepotism expression in organizations of Lithuania." Organizacijø Vadyba: Sisteminiai Tyrimai(66): 129.

Wadongo, B. and M. Abdel-Kader (2014). "Contingency theory, performance management and organisational effectiveness in the third sector: A theoretical framework." International journal of productivity and performance management 63(6): 680-703.

Walker, R. M., et al. (2010). "Management innovation and organizational performance: The mediating effect of performance management." Journal of Public Administration Research and Theory 21(2): 367386.

Walsh, K. and D. Fisher (2005). "Action inquiry and performance appraisals: Tools for organizational learning and development." The Learning Organization 12(1): 26-41.

Walsh, M. B. (2003). Perceived fairness of and satisfaction with employee performance appraisal, Doctoral Dissertation, Louisiana State University.

Wang, X. (2002). "Assessing Administrative Accountability Results from a National Survey." The American Review of Public Administration 32(3): 350-370.

Warner, M. (2010). "In search of Confucian HRM: theory and practice in Greater China and beyond." The International Journal of Human Resource Management 21(12): 2053-2078. 
Weibel, A., et al. (2009). "Pay for performance in the public sector-Benefits and (hidden) costs." Journal of Public Administration Research and Theory 20(2): 387-412.

West, D. and D. Blackman (2015). "Performance management in the public sector." Australian Journal of Public Administration 74(1): 73-81.

Wexley, K. N. (1984). "Personnel training." Annual review of psychology 35(1): 519-551.

Wheaton, B., et al. (1977). "Assessing reliability and stability in panel models." $\underline{\text { Sociological }}$ methodology 8: 84-136.

Wheeless, L. R., et al. (1984). "The relationships of communication with supervisor and decisionparticipation to employee job satisfaction." Communication Quarterly 32(3): 222-232.

Whitman, D. S., et al. (2010). "Satisfaction, citizenship behaviors, and performance in work units: A meta-analysis of collective construct relations." Personnel Psychology 63(1): 41-81.

Wiese, D. S. and M. R. Buckley (1998). "The evolution of the performance appraisal process." Journal of management History 4(3): 233-249.

Williams, R. S. (1998). Performance management: Perspectives on employee performance, International Thomson Business Press, Boston, MA.

Woehr, D. J. and A. I. Huffcutt (1994). "Rater training for performance appraisal: A quantitative review." Journal of Occupational and Organizational Psychology 67(3): 189-205.

Wood, R. E. and V. Marshall (2008). "Accuracy and effectiveness in appraisal outcomes: the influence of self-efficacy, personal factors and organisational variables." Human Resource Management Journal 18(3): 295-313.

Wright, B. E. (2004). "The role of work context in work motivation: A public sector application of goal and social cognitive theories." Journal of Public Administration Research and Theory 14(1): 59-78.

Wright, K. B. (2005). "Researching Internet-based populations: Advantages and disadvantages of online survey research, online questionnaire authoring software packages, and web survey services." Journal of Computer-Mediated Communication 10(3): http://jcmc.indiana.edu/vol10/issue13/wright.html.

Yang, K. and S. K. Pandey (2009). "How do perceived political environment and administrative reform affect employee commitment?" Journal of Public Administration Research and Theory 19(2): 335-360.

Yeganeh, H. and Z. Su (2008). "An examination of human resource management practices in Iranian public sector." Personnel Review 37(2): 203-221.

Yi, Z. (2013). "Setting goals for change in the information age: Approaches of academic library directors in the USA." Library Management 34(1/2): 5-19.

Yin, R. K. (2003). Case study research: Design and methods 3rd ed, London, Sage publications.

Yin, R. K. (2009). Case Study Research, Design \& Methods 4th ed, London and Singapore, Sage publications.

Yuksel, Y. (2013). Understanding the role of culture and communication in implementing planned organizational change: the case of Compstat in police organizations, Rutgers The State University of New Jersey-New Brunswick. 
Yusof, M. and K. K. Jain (2009). "Entrepreneurial leadership and academic entrepreneurship in Malaysian public research universities." Asia Pacific Journal of Innovation and Entrepreneurship 3(3): 63-84.

Zahra, S. A. and J. G. Covin (1995). "Contextual influences on the corporate entrepreneurshipperformance relationship: A longitudinal analysis." Journal of Business Venturing 10(1): 43-58.

Zedeck, S. (1971). "Problems with the use of" moderator" variables." Psychological bulletin 76(4): 295.

Zhu, Y., et al. (2007). "Human resource management with 'Asian'characteristics: a hybrid peoplemanagement system in East Asia." The International Journal of Human Resource Management 18(5): 745-768.

Zientara, P. and G. Kuczyński (2009). "Human resources practices and work-related attitudes in Polish public administration." Eastern European Economics 47(5): 42-60.

Zupan, N. and R. Kaše (2005). "Strategic human resource management in European transition economies: building a conceptual model on the case of Slovenia." The International Journal of Human Resource Management 16(6): 882-906. 


\title{
Appendices
}

\section{Appendix A: Questionnaire in English}

\author{
TE WHARE WÃNANGA O TE OPOKO O TE IKA A MĀUI \\ 59 \\ \&

\section{SURVEY OF FACTORS INFLUENCING PERFORMANCE APPRAISAL AND MANAGEMENT IN THE VIETNAM PUBLIC SECTOR}

\section{Dear Sir or Madam}

We would like to thank you for participating in a survey in order to identify factors that affect the effectiveness of the current performance appraisal and management system in the Vietnam public sector.

No personally identifying information will be collected to ensure the anonymity of respondents. All material collected will be kept confidential, and will be viewed only by my supervisors and myself. This information only serves for scientific research purposes without any other purpose.

The survey will take about 20 minutes to compete. Please return the completed survey via the addressed and prepaid reply envelope by 30 November 2016.

Please contact the researcher as the information listed below if you have any questions about the research.

We highly appreciate your participation in this survey and look forward to receiving your feedback.

Thank you!

\section{Yours sincerely \\ Tai Anh Vu \\ PhD Student}

School of Management

Victoria University of Wellington

Room RWW 531, Wellington 6140, New Zealand

Phone in New Zealand: + 64-4-463 8632

Mobile in Vietnam: 0904314387

Email: TaiAnh.Vu@vuw.ac.nz or vuanhtai07@gmail.com 


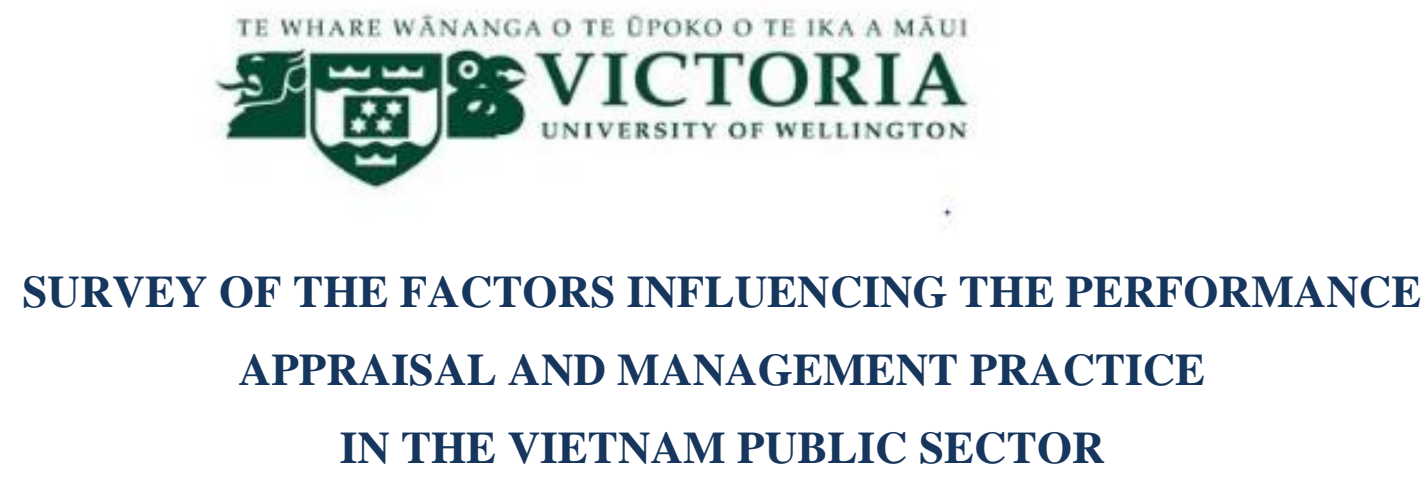

The following questions relate to your personal and work status

$\begin{array}{lll}\text { Highest qualification: } & \text { Doctor } \square & \text { Master } \\ & \text { Bachelor } \square & \text { Others }\end{array}$

Total years working in the public sector:

Gender:

Age:

Organisational level:

Organisational category: Policy-making

40 to under 50

Central agency

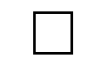

30 to under 40

Over 50

Provincial agency

Service delivery

Partially self-financing

Don't know
Your organisation's degree

of self-financing
Totally self-financing $\square$
Totally subsidised
by the state
Your position in the

organisation:
Departmental manager

None manager

If other, please state specifically:
Divisional manager

Others 
In the following sections, we are interested in your perceptions about the performance appraisal and management practices in your organisation. Please indicate your answer by circling the number you agree with. There is no right or wrong answer. The best answer is the answer that expresses your honest and objective perception on the current system.

To what extent do you disagree or agree with the following statements regarding performance appraisal and management practices being currently used in your organisation?

\begin{tabular}{|c|c|c|c|c|c|c|c|}
\hline & Strongly & Basically & Slightly & Neither & Slightly & Basically & Strongly \\
\hline & Disagree & disagree & disagree & $\begin{array}{l}\text { agree nor } \\
\text { disagree }\end{array}$ & agree & agree & Agree \\
\hline $\begin{array}{l}\text { My evaluation is totally based on the work goals I } \\
\text { accomplished. }\end{array}$ & 1 & 2 & 3 & 4 & 5 & 6 & 7 \\
\hline My evaluation are based on my skills and abilities. & 1 & 2 & 3 & 4 & 5 & 6 & 7 \\
\hline $\begin{array}{l}\text { All my performance appraisal criteria are measurable and } \\
\text { objective. }\end{array}$ & 1 & 2 & 3 & 4 & 5 & 6 & 7 \\
\hline My supervisor gives me timely feedback. & 1 & 2 & 3 & 4 & 5 & 6 & 7 \\
\hline $\begin{array}{l}\text { My supervisor gives me specific feedback about what is } \\
\text { good and bad about my performance. }\end{array}$ & 1 & 2 & 3 & 4 & 5 & 6 & 7 \\
\hline My supervisor gives me honest feedback. & 1 & 2 & 3 & 4 & 5 & 6 & 7 \\
\hline $\begin{array}{l}\text { There is a close tie between appraisal results and pay (extra } \\
\text { income) in my organisation. }\end{array}$ & 1 & 2 & 3 & 4 & 5 & 6 & 7 \\
\hline There is a close tie between appraisal results and rewards. & 1 & 2 & 3 & 4 & 5 & 6 & 7 \\
\hline My rewards reflect the effort I have put into my work. & 1 & 2 & 3 & 4 & 5 & 6 & 7 \\
\hline $\begin{array}{l}\text { In my organisation, the employees having poor } \\
\text { performance will be removed to other jobs. }\end{array}$ & 1 & 2 & 3 & 4 & 5 & 6 & 7 \\
\hline I can openly discuss job problems with my supervisor. & 1 & 2 & 3 & 4 & 5 & 6 & 7 \\
\hline $\begin{array}{l}\text { I feel comfortable in expressing my opinion about the } \\
\text { rating result to the supervisor if it is unreasonable. }\end{array}$ & 1 & 2 & 3 & 4 & 5 & 6 & 7 \\
\hline
\end{tabular}

To what extent do you disagree or agree with the following statements about the accountability of the chief executive for the organisation's performance? 


\begin{tabular}{|l|c|c|c|c|c|c|c|}
\hline & $\begin{array}{c}\text { Strongly } \\
\text { Disagre }\end{array}$ & $\begin{array}{c}\text { Basically } \\
\text { disagree }\end{array}$ & $\begin{array}{c}\text { Slightly } \\
\text { disagree }\end{array}$ & $\begin{array}{c}\text { Neither } \\
\text { agree nor } \\
\text { disagree }\end{array}$ & $\begin{array}{c}\text { Slightly } \\
\text { agree }\end{array}$ & $\begin{array}{c}\text { Basically } \\
\text { agree }\end{array}$ & $\begin{array}{c}\text { Strongly } \\
\text { Agree }\end{array}$ \\
\hline $\begin{array}{l}\text { Annually, my organisation must register key assignments (goals) } \\
\text { for approval from upper agencies. }\end{array}$ & 1 & 2 & 3 & 4 & 5 & 6 & 7 \\
\hline $\begin{array}{l}\text { My organisation's performance is evaluated by agreed specific } \\
\text { indicators. }\end{array}$ & 1 & 2 & 3 & 4 & 5 & 6 & 7 \\
\hline $\begin{array}{l}\text { My chief executive receives proper recognition and rewards } \\
\text { corresponding to excellent performance. }\end{array}$ & 1 & 2 & 3 & 4 & 5 & 6 & 7 \\
\hline $\begin{array}{l}\text { My organisation's performance is rated and compared with other } \\
\text { institutions. }\end{array}$ & 1 & 2 & 3 & 4 & 5 & 6 & 7 \\
\hline $\begin{array}{l}\text { My organisation's performance is seriously and thoroughly } \\
\text { evaluated by upper agencies. }\end{array}$ & 1 & 2 & 3 & 4 & 5 & 6 & 7 \\
\hline $\begin{array}{l}\text { My chief executive receives criticism when the organisation does } \\
\text { not achieve the agreed goals. }\end{array}$ & 1 & 2 & 3 & 4 & 5 & 6 & 7 \\
\hline
\end{tabular}

What extent of autonomy does your organisation really have in the following personnel management practices?

\begin{tabular}{|c|c|c|c|c|c|}
\hline & $\begin{array}{c}\text { Not at } \\
\text { all }\end{array}$ & Very low & $\begin{array}{c}\text { Some } \\
\text { autonomy }\end{array}$ & $\begin{array}{c}\text { Great deal of } \\
\text { autonomy }\end{array}$ & $\begin{array}{l}\text { Totally } \\
\text { autonomy }\end{array}$ \\
\hline in personnel hiring? & 1 & 2 & 3 & 4 & 5 \\
\hline in determining pay or bonus amounts? & 1 & 2 & 3 & 4 & 5 \\
\hline in promotion? & 1 & 2 & 3 & 4 & 5 \\
\hline in placing and assigning staff? & 1 & 2 & 3 & 4 & 5 \\
\hline in transferring unsuitable staff? & 1 & 2 & 3 & 4 & 5 \\
\hline in discharging unsuitable staff? & 1 & 2 & 3 & 4 & 5 \\
\hline
\end{tabular}

To what extent do you disagree or agree with the following statements?

\begin{tabular}{|l|c|c|c|c|c|c|c|}
\hline & $\begin{array}{c}\text { Strongly } \\
\text { Disagre }\end{array}$ & $\begin{array}{c}\text { Basically } \\
\text { disagree }\end{array}$ & $\begin{array}{c}\text { Slightly } \\
\text { disagree }\end{array}$ & $\begin{array}{c}\text { Neither } \\
\text { agree nor } \\
\text { disagree }\end{array}$ & $\begin{array}{c}\text { Slightly } \\
\text { agree }\end{array}$ & $\begin{array}{c}\text { Basically } \\
\text { agree }\end{array}$ & $\begin{array}{c}\text { Strongly } \\
\text { Agree }\end{array}$ \\
\hline My chief executive & & & & & & & \\
\hline $\begin{array}{l}\text { often comes up with radical improvement ideas to enhance } \\
\text { my institution's operational effectiveness. }\end{array}$ & 1 & 2 & 3 & 4 & 5 & 6 & 7 \\
\hline
\end{tabular}




\begin{tabular}{|l|c|c|c|c|c|c|c|}
\hline is willing to take risks in his decisions. & 1 & 2 & 3 & 4 & 5 & 6 & 7 \\
\hline demonstrates the passion for his/her work. & 1 & 2 & 3 & 4 & 5 & 6 & 7 \\
\hline has a vision of the future of our organisation. & 1 & 2 & 3 & 4 & 5 & 6 & 7 \\
\hline $\begin{array}{l}\text { challenges and pushes us to act in a more innovative way. } \\
\text { is patient in pursuing new improvement and solutions. }\end{array}$ & 1 & 2 & 3 & 4 & 5 & 6 & 7 \\
\hline
\end{tabular}

\section{To what extent to you disagree or agree with the following statements about the HR unit expertise}

\begin{tabular}{|c|c|c|c|c|c|c|c|}
\hline & $\begin{array}{l}\text { Strongly } \\
\text { Disagree }\end{array}$ & $\begin{array}{r}\text { Basically } \\
\text { disagree }\end{array}$ & $\begin{array}{l}\text { Slightly } \\
\text { disagree }\end{array}$ & $\begin{array}{c}\text { Neither } \\
\text { agree nor } \\
\text { disagree }\end{array}$ & \begin{tabular}{c|c} 
Slightly \\
agree
\end{tabular} & $\begin{array}{c}\text { Basically } \\
\text { agree }\end{array}$ & $\begin{array}{c}\text { Strongly } \\
\text { Agree }\end{array}$ \\
\hline \multicolumn{8}{|l|}{ The HR professionals in our organisation } \\
\hline $\begin{array}{l}\text { have enough expertise to develop and organise an effective goal- } \\
\text { based appraisal system. }\end{array}$ & 1 & 2 & 3 & 4 & 5 & 6 & 7 \\
\hline can develop clear and specific performance standards. & 1 & 2 & 3 & 4 & 5 & 6 & 7 \\
\hline $\begin{array}{l}\text { are competent to develop performance appraisal systems that } \\
\text { differentiate between employee performance levels fairly and } \\
\text { impartially. }\end{array}$ & 1 & 2 & 3 & 4 & 5 & 6 & 7 \\
\hline are competent to develop a sound pay-for-performance system. & 1 & 2 & 3 & 4 & 5 & 6 & 7 \\
\hline $\begin{array}{l}\text { can provide line managers with valuable insights or useful advice } \\
\text { regarding personnel management. }\end{array}$ & 1 & 2 & 3 & 4 & 5 & 6 & 7 \\
\hline have strong HR field expertise. & 1 & 2 & 3 & 4 & 5 & 6 & 7 \\
\hline
\end{tabular}

\section{To what extent do you disagree or agree with the following statements?}

\begin{tabular}{|l|c|c|c|c|c|c|c|}
\hline & $\begin{array}{c}\text { Strongly } \\
\text { Disagree }\end{array}$ & $\begin{array}{c}\text { Basically } \\
\text { disagree }\end{array}$ & $\begin{array}{c}\text { Slightly } \\
\text { disagree }\end{array}$ & $\begin{array}{c}\text { Neither } \\
\text { agree nor } \\
\text { disagree }\end{array}$ & $\begin{array}{c}\text { Slightly } \\
\text { agree }\end{array}$ & $\begin{array}{c}\text { Basically } \\
\text { agree }\end{array}$ & $\begin{array}{c}\text { Strongly } \\
\text { Agree }\end{array}$ \\
\hline In my workplace, & & & & & & & \\
\hline $\begin{array}{l}\text { a number of people joined my institution through personal } \\
\text { relationships. }\end{array}$ & 1 & 2 & 3 & 4 & 5 & 6 & 7 \\
\hline $\begin{array}{l}\text { those who have a good relationship with their supervisor will } \\
\text { get better treatment in jobs compared to others. }\end{array}$ & 1 & 2 & 3 & 4 & 5 & 6 & 7 \\
\hline
\end{tabular}




\begin{tabular}{|l|l|l|l|l|l|l|l|}
\hline $\begin{array}{l}\text { a person is more likely to be promoted when they have a } \\
\text { good relationship with the leader of the organisation. }\end{array}$ & 2 & 3 & 4 & 5 & 6 & 7 \\
\hline $\begin{array}{l}\text { supervisors find it difficult to be completely objective in } \\
\text { performance appraisals because they have an interest in } \\
\text { maintaining good relationships with their employees. }\end{array}$ & 1 & 2 & 3 & 4 & 5 & 6 & 7 \\
\hline $\begin{array}{l}\text { participating in interesting training with limited quota is } \\
\text { often affected by the interpersonal factor. }\end{array}$ & 1 & 2 & 3 & 4 & 5 & 6 & 7 \\
\hline
\end{tabular}

To what extent do you disagree or agree with the following statements?

\begin{tabular}{|c|c|c|c|c|c|c|c|}
\hline & \begin{tabular}{l|} 
Strongly \\
Disagree
\end{tabular} & $\begin{array}{c}\text { Basically } \\
\text { disagree }\end{array}$ & $\begin{array}{l}\text { Slightly } \\
\text { disagree }\end{array}$ & \begin{tabular}{|c} 
Neither \\
agree nor \\
disagree
\end{tabular} & $\begin{array}{c}\text { Slightly } \\
\text { agree }\end{array}$ & $\begin{array}{c}\text { Basically } \\
\text { agree }\end{array}$ & $\begin{array}{c}\text { Strongly } \\
\text { Agree }\end{array}$ \\
\hline \multicolumn{8}{|l|}{ In my organisation, } \\
\hline $\begin{array}{l}\text { supervisors are afraid of subordinates who are related to } \\
\text { high-level executives. }\end{array}$ & 1 & 2 & 3 & 4 & 5 & 6 & 7 \\
\hline promotion is affected by kinship relationships. & 1 & 2 & 3 & 4 & 5 & 6 & 7 \\
\hline $\begin{array}{l}\text { promotion or rewarding based on family ties has a negative } \\
\text { influence on employee motivation. }\end{array}$ & 1 & 2 & 3 & 4 & 5 & 6 & 7 \\
\hline $\begin{array}{l}\text { leaders' relatives are often promoted to good job positions, } \\
\text { though their competence is not as good as others. }\end{array}$ & 1 & 2 & 3 & 4 & 5 & 6 & 7 \\
\hline $\begin{array}{l}\text { ability, knowledge and skill are less important than kinship } \\
\text { relationships in the promotion process. }\end{array}$ & 1 & 2 & 3 & 4 & 5 & 6 & 7 \\
\hline
\end{tabular}

To what extent to you disagree or agree with the following statements about the supporting activities for the performance appraisal and management

\begin{tabular}{|l|c|c|c|c|c|c|c|}
\hline & $\begin{array}{c}\text { Strongly } \\
\text { Disagre }\end{array}$ & $\begin{array}{c}\text { Basically } \\
\text { disagree }\end{array}$ & $\begin{array}{c}\text { Slightly } \\
\text { disagree }\end{array}$ & $\begin{array}{c}\text { Neither } \\
\text { agree nor } \\
\text { disagree }\end{array}$ & $\begin{array}{c}\text { Slightly } \\
\text { agree }\end{array}$ & $\begin{array}{c}\text { Basically } \\
\text { agree }\end{array}$ & $\begin{array}{c}\text { Strongly } \\
\text { Agree }\end{array}$ \\
\hline $\begin{array}{l}\text { My organisation has clearly explained the necessity of } \\
\text { introducing the performance management system. }\end{array}$ & 1 & 2 & 3 & 4 & 5 & 6 & 7 \\
\hline $\begin{array}{l}\text { Our leaders have encouraged all of us to embrace the } \\
\text { performance appraisal system. }\end{array}$ & 1 & 2 & 3 & 4 & 5 & 6 & 7 \\
\hline $\begin{array}{l}\text { We are sufficiently informed about progress around the } \\
\text { introduction of the performance management system. }\end{array}$ & 1 & 2 & 3 & 4 & 5 & 6 & 7 \\
\hline $\begin{array}{l}\text { Two-way communication between senior managers and } \\
\text { employees is very open and democratic. }\end{array}$ & 1 & 2 & 3 & 4 & 5 & 6 & 7 \\
\hline $\begin{array}{l}\text { Policies and procedures regarding the performance } \\
\text { management system are clearly communicated. }\end{array}$ & 1 & 2 & 3 & 4 & 5 & 6 & 7 \\
\hline
\end{tabular}


We have received adequate training on performance management.

To what extent to you disagree or agree with the following statements

\begin{tabular}{|c|c|c|c|c|c|c|c|}
\hline & $\begin{array}{l}\text { Strongly } \\
\text { Disagree }\end{array}$ & $\begin{array}{r}\text { Basically } \\
\text { disagree }\end{array}$ & $\begin{array}{l}\text { Slightly } \\
\text { disagree }\end{array}$ & $\begin{array}{c}\text { Neither } \\
\text { agree nor } \\
\text { disagree }\end{array}$ & $\begin{array}{c}\text { Slightly } \\
\text { agree }\end{array}$ & $\begin{array}{c}\text { Basically } \\
\text { agree }\end{array}$ & $\begin{array}{c}\text { Strongly } \\
\text { Agree }\end{array}$ \\
\hline $\begin{array}{l}\text { I put forth my best effort to get my job done regardless of } \\
\text { the difficulties. }\end{array}$ & 1 & 2 & 3 & 4 & 5 & 6 & 7 \\
\hline I am willing to start work early or stay late to finish a job. & 1 & 2 & 3 & 4 & 5 & 6 & 7 \\
\hline I do extra work for my job that isn't really expected of me. & 1 & 2 & 3 & 4 & 5 & 6 & 7 \\
\hline I emphasise doing my job right the first time. & 1 & 2 & 3 & 4 & 5 & 6 & 7 \\
\hline $\begin{array}{l}\text { I am motivated to put in as much effort into my job as I } \\
\text { possibly can. }\end{array}$ & 1 & 2 & 3 & 4 & 5 & 6 & 7 \\
\hline I feel a strong sense of belonging to my organisation & 1 & 2 & 3 & 4 & 5 & 6 & 7 \\
\hline I feel personally attached to my work organisation. & 1 & 2 & 3 & 4 & 5 & 6 & 7 \\
\hline $\begin{array}{l}\text { Working at my organisation has a great deal of personal } \\
\text { meaning for me. }\end{array}$ & 1 & 2 & 3 & 4 & 5 & 6 & 7 \\
\hline I would be happy to work at my organisation until I retire. & 1 & 2 & 3 & 4 & 5 & 6 & 7 \\
\hline $\begin{array}{l}\text { I really feel that the problems faced by my organisation are } \\
\text { also my problems. }\end{array}$ & 1 & 2 & 3 & 4 & 5 & 6 & 7 \\
\hline In general, I am satisfied with my job. & 1 & 2 & 3 & 4 & 5 & 6 & 7 \\
\hline
\end{tabular}

Please indicate to what extent your organisation is effective in accomplishing its goals and mission

\begin{tabular}{|l|c|c|c|c|c|c|c|}
\hline & $\begin{array}{c}\text { Strongly } \\
\text { Disagree }\end{array}$ & $\begin{array}{c}\text { Basically } \\
\text { disagree }\end{array}$ & $\begin{array}{c}\text { Slightly } \\
\text { disagree }\end{array}$ & $\begin{array}{c}\text { Neither } \\
\text { agree nor } \\
\text { disagree }\end{array}$ & $\begin{array}{c}\text { Slightly } \\
\text { agree }\end{array}$ & $\begin{array}{c}\text { Basically } \\
\text { agree }\end{array}$ & $\begin{array}{c}\text { Strongly } \\
\text { Agree }\end{array}$ \\
\hline This organisation is achieving its full potential. & 1 & 2 & 3 & 4 & 5 & 6 & 7 \\
\hline $\begin{array}{l}\text { People at my level are satisfied with this organisation's } \\
\text { performance. }\end{array}$ & 1 & 2 & 3 & 4 & 5 & 6 & 7 \\
\hline
\end{tabular}




\begin{tabular}{|l|l|l|l|l|l|l|l|}
\hline $\begin{array}{l}\text { This organisation does a good job of satisfying its clients, } \\
\text { same-level agencies and upper-leaders. }\end{array}$ & 2 & 3 & 4 & 5 & 6 & 7 \\
\hline $\begin{array}{l}\text { This organisation gives me the opportunity and } \\
\text { encouragement to do the best work I am capable of. }\end{array}$ & 1 & 2 & 3 & 4 & 5 & 6 & 7 \\
\hline $\begin{array}{l}\text { The upper leaders and same-level agencies highly appreciate } \\
\text { our organisation's performance. }\end{array}$ & 1 & 2 & 3 & 4 & 5 & 6 & 7 \\
\hline
\end{tabular}

In your opinion, what are the most important things affecting the effectiveness of the performance appraisal and management system being implemented in your organisation?

In your opinion, what should be done to improve public employee performance in the future?

Thank you for taking time to fill out this survey. Your contribution will absolutely help us to complete the present research project.

Please return the questionnaire in the addressed and pre-paid reply envelope provided by 30 October 2016. 
TE WHARE WÃNANGA O TE OPOKO O TE IKA A MĀUI 59

1 U

\title{
KHẢO SÁT CÁC NHÂN TỔ ẢNH HƯởNG ĐẾN CÔNG TÁC ĐÁNH GIÁ VÀ QUẢN LÝ KẾT QUẢ THỰC HIỆN NHIỆM VỤ CỦA CÔNG CHỨC, VIÊN CHỨC
}

\section{Kính thưa: Ông/ bà....}

Chúng tôi chân thành cảm ơn ông/bà đã quyết định sẽ dành thời gian hỗ trợ nghiên cứu khảo sát hiện trạng các nhân tố ảnh hưởng tới công tác đánh giá và quản lý kết quả thực hiện nhiệm vụ của công chức, viên chức. Nghiên cứu được thực hiện để giúp các nhà nghiên cứu và các nhà xây dựng chính sách có sự hiểu biết đầy đủ hơn về công tác đánh giá hiện hành và đề xuất những biện pháp để thực hiện có hiệu quả công tác này trong tương lai.

Sẽ không có thông tin cá nhân riêng của ông/bà được thu thập để bảo đảm tính bảo mật của người tham gia. Tất cả thông tin thu thập sẽ được giữ bí mật và chỉ phục vụ cho mục đích nghiên cứu khoa học mà không vì mục đích nào khác.

Việc trả lời phiếu khảo sát sẽ diễn ra khoảng 20 phút. Sau khi hoàn thành, xin ông/ bà vui lòng gửi lại phiếu khảo sát đã được hoàn thành tới địa chỉ ghi tại phong bì được gửi kèm theo trước ngày 30 tháng 11 năm 2016.

Trong trường hợp có thông tin chưa rõ, xin ông/bà vui lòng liên hệ với người nghiên cứu theo số điện thoại và địa chỉ liên hệ như dưới đây.

Chúng tôi đánh giá cao sự tham gia và mong muốn nhận được phiếu phản hồi của ông/bà.

Xin trân trọng cảm ơn!

\author{
Vũ Anh Tài \\ Nghiên cứu sinh \\ Khoa Quản lý, Đại học Victoria \\ University of Wellington \\ Văn phòng: RWW 531, Wellington \\ 6140, New Zealand \\ Điện thoại ở NZ: + 64-4-463 8632 \\ Điện thoại ở Việt Nam: 0904314387 \\ Email: TaiAnh.Vu@vuw.ac.nz hoặc \\ vuanhtai07@gmail.com
}




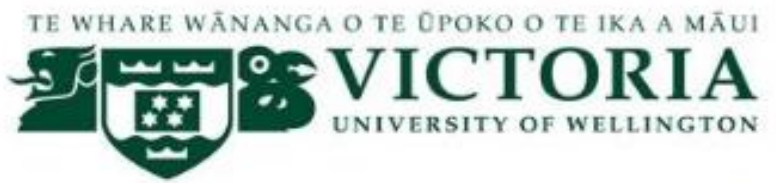

\section{KHẢO SÁT CÁC NHÂN TỐ ẢNH HƯởNG ĐẾN CÔNG TÁC ĐÁNH GIÁ VÀ QUẢN LÝ KẾT QUẢ THỤ̉C HIỆN NHIỆM VỤ CỦA CÔNG CHỬC, VIÊN CHỬC}

Câu hỏi dưới đây liên quan đến một số thông tin chung về bản thân và công việc của ông/bà

\begin{tabular}{lll} 
Trình độ học vấn & Tiến sĩ & Thạc sĩ \\
cao nhất: & Cử nhân/ Kỹ sư $\square$ & Trình độ khác \\
\hline
\end{tabular}

Tổng số năm làm việc

trong khu vực nhà nước

Giới tính:

Độ tuổi:

Cấp độ tổ chức:

Cơ quan ông bà đang

công tác là:

Mức độ tự chủ tài chính: Tự chủ hoàn toàn

Ngân sách chi trả

toàn bộ

Vị trí hoặc chức vụ hiện tại của ông/bà:

Dưới 5 năm

Dưới 30
Từ 15 đến dưới 20

$\square$

Tù̀ 5 đến dưới $10 \square$
Từ 20 đến dưới $25 \square$

Từ 10 đến dưới 15

Từ 25 năm trở lên

Nữ

Từ 40 đến dưới 50

Từ 30 đến dưới 40

Trên 50

Cơ quan trung ương

Cơ quan cấp tỉnh

Đơn vị sự nghiệp

Tự chủ một phần

Không biết

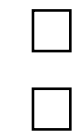

Lãnh đạo cấp phòng và tương đương

Khác

Nếu là khác, xin vui lòng ghi rõ: . 
Trong các phần dưới đây, chúng tôi mong muốn nhận được ý kiến của ông/bà về thực trạng công tác đánh giá và quản lý kết quả thực hiện nhiệm vụ ở cơ quan ông/bà. Xin vui lòng chỉ ra ý kiến của ông/bà bằng cách khoanh tròn vào số phù hợp nhất của mỗi phát biểu dưới đây. Không có câu trả lời đúng hoặc sai. Câu trả lời tốt nhất là câu trả lời phản ánh một cách trung thực và khách quan nhất quan sát và nhận xét của ông/bà về hiện trạng công tác này ở cơ quan mình.

Mức độ nào ông/bà đồng ý hoặc không đồng ý với các phát biểu dưới đây về công tác đánh giá và quản lý kết quả thực hiện nhiệm vụ hiện đang được thực hiện ở cơ quan mình?

\begin{tabular}{|c|c|c|c|c|c|c|c|}
\hline & $\begin{array}{c}\text { Rất } \\
\text { không } \\
\text { đồng ý }\end{array}$ & $\begin{array}{l}\text { Cơ bản } \\
\text { không } \\
\text { đồng ý }\end{array}$ & $\begin{array}{l}\text { Hơi } \\
\text { không } \\
\text { đồng ý }\end{array}$ & $\begin{array}{c}\text { Không } \\
\text { đồng ý } \\
\text { cũng } \\
\text { không } \\
\text { phản } \\
\text { đối }\end{array}$ & $\begin{array}{l}\text { Hơi } \\
\text { đồng ý }\end{array}$ & $\begin{array}{l}\text { Cơ bản } \\
\text { đồng ý }\end{array}$ & $\begin{array}{l}\text { Rất } \\
\text { đồng } \text {. }\end{array}$ \\
\hline $\begin{array}{l}\text { Kết quả đánh giá, phân loại của tôi hoàn toàn được } \\
\text { dựa trên kết quả công việc thực tế tôi đã hoàn } \\
\text { thành }\end{array}$ & 1 & 2 & 3 & 4 & 5 & 6 & 7 \\
\hline $\begin{array}{l}\text { Kết quả đánh giá, phân loại của tôi hoàn toàn được } \\
\text { dựa trên năng lực và kiến thức của tôi }\end{array}$ & 1 & 2 & 3 & 4 & 5 & 6 & 7 \\
\hline $\begin{array}{l}\text { Tất cả các tiêu chí sử dụng để đánh giá tôi là khách } \\
\text { quan và có thể đo lường, định lượng }\end{array}$ & 1 & 2 & 3 & 4 & 5 & 6 & 7 \\
\hline $\begin{array}{l}\text { Cấp trên luôn góp ý cho tôi về thực hiện công việc } \\
\text { một cách kịp thời }\end{array}$ & 1 & 2 & 3 & 4 & 5 & 6 & 7 \\
\hline $\begin{array}{l}\text { Cấp trên góp ý cho tôi một cách cụ thể về những } \\
\text { mặt được và chưa được liên quan đến công việc } \\
\text { của tôi }\end{array}$ & 1 & 2 & 3 & 4 & 5 & 6 & 7 \\
\hline Cấp trên góp ý cho tôi một cách chân thành & 1 & 2 & 3 & 4 & 5 & 6 & 7 \\
\hline $\begin{array}{l}\text { Ở cơ quan tôi, tiền lương hoặc thu thập tăng thêm } \\
\text { được gắn kết chặt chẽ với kết quả đánh giá }\end{array}$ & 1 & 2 & 3 & 4 & 5 & 6 & 7 \\
\hline $\begin{array}{l}\text { Khen thưởng và chế độ đãi ngộ của tôi gắn bó chặt } \\
\text { chẽ với kết quả đánh giá }\end{array}$ & 1 & 2 & 3 & 4 & 5 & 6 & 7 \\
\hline $\begin{array}{l}\text { Khen thưởng và chế độ đãi ngộ phản ánh những } \\
\text { nỗ lực tôi đã dành cho công việc của tôi }\end{array}$ & 1 & 2 & 3 & 4 & 5 & 6 & 7 \\
\hline $\begin{array}{l}\text { Ơ cơ quan tôi, những người không hoàn thành } \\
\text { nhiệm vụ sẽ bị điều chuyển đến vị trí khác phù } \\
\text { hợp với năng lực hơn }\end{array}$ & 1 & 2 & 3 & 4 & 5 & 6 & 7 \\
\hline $\begin{array}{l}\text { Tôi có thể trao đổi một cách cởi mở các vấn đề } \\
\text { liên quan đến công việc với cấp trên của tôi }\end{array}$ & 1 & 2 & 3 & 4 & 5 & 6 & 7 \\
\hline
\end{tabular}




\begin{tabular}{|l|l|l|l|l|l|l|l|}
\hline $\begin{array}{l}\text { Tôi có thể thoải mái trình bày quan điểm của mình } \\
\text { về kết quả đánh giá với lãnh đạo đơn vị, nếu thấy } \\
\text { không hợp lý }\end{array}$ & 1 & 2 & 3 & 4 & 5 & 6 & 7 \\
\hline
\end{tabular}

Mức độ nào ông/bà đồng ý hoặc không đồng ý với phát biểu dưới đây về thực trạng trách nhiệm giải trình của thủ trưởng cơ quan mình đối với kết quả thực hiện nhiệm vụ của cơ quan?

\begin{tabular}{|c|c|c|c|c|c|c|c|}
\hline & $\begin{array}{l}\text { Rất } \\
\text { không } \\
\text { đồng ý }\end{array}$ & $\begin{array}{l}\text { Cơ } \\
\text { bản } \\
\text { không } \\
\text { đồng ý }\end{array}$ & $\begin{array}{l}\text { Hơi } \\
\text { không } \\
\text { đồng ý }\end{array}$ & $\begin{array}{c}\text { Không } \\
\text { đồng ý } \\
\text { cũng } \\
\text { không } \\
\text { phản } \\
\text { đối }\end{array}$ & $\begin{array}{l}\text { Hoi } \\
\text { đồng ý }\end{array}$ & $\begin{array}{l}\text { Cơ } \\
\text { bản } \\
\text { đồng ý }\end{array}$ & $\begin{array}{l}\text { Rất } \\
\text { đồng ý }\end{array}$ \\
\hline $\begin{array}{l}\text { Hàng năm cơ quan tôi phải đăng ký các nhiệm vụ } \\
\text { trọng tâm với cơ quan cấp trên để được phê duyệt } \\
\text { trước khi thực hiện }\end{array}$ & 1 & 2 & 3 & 4 & 5 & 6 & 7 \\
\hline $\begin{array}{l}\text { Kết quả thực hiện nhiệm vụ của cơ quan tôi được } \\
\text { đánh giá bởi các tiêu chí đã được quy định cụ thể }\end{array}$ & 1 & 2 & 3 & 4 & 5 & 6 & 7 \\
\hline $\begin{array}{l}\text { Thủ trưởng cơ quan tôi nhận được phần thưởng } \\
\text { xứng đáng nếu cơ quan hoàn thành xuất sắc nhiệm } \\
\text { vụ đã đăng ký }\end{array}$ & 1 & 2 & 3 & 4 & 5 & 6 & 7 \\
\hline $\begin{array}{l}\text { Kết quả thực hiện nhiệm vụ của cơ quan tôi được } \\
\text { đánh giá, phân loại và so sánh với các cơ quan, đơn } \\
\text { vị khác }\end{array}$ & 1 & 2 & 3 & 4 & 5 & 6 & 7 \\
\hline $\begin{array}{l}\text { Kết quả thực hiện nhiệm vụ của cơ quan tôi được } \\
\text { đánh giá một cách nghiêm túc và kỹ lưỡng bởi cơ } \\
\text { quan cấp trên }\end{array}$ & 1 & 2 & 3 & 4 & 5 & 6 & 7 \\
\hline $\begin{array}{l}\text { Thủ trưởng cơ quan tôi bị phê bình nếu cơ quan } \\
\text { không đạt được các mục tiêu đã đăng ký }\end{array}$ & 1 & 2 & 3 & 4 & 5 & 6 & 7 \\
\hline
\end{tabular}

Ông bà đồng ý ở mức độ nào về quyền tự chủ thực sự của cơ quan mình trong các công tác quản lý nhân sự dưới đây?

\begin{tabular}{|l|c|c|c|c|c|}
\hline & $\begin{array}{c}\text { Gần như } \\
\text { không có } \\
\text { tự chủ }\end{array}$ & $\begin{array}{c}\text { Ở mức độ } \\
\text { tự chú rât } \\
\text { thấp }\end{array}$ & $\begin{array}{c}\text { Có một số } \\
\text { quyề tự } \\
\text { chủ }\end{array}$ & $\begin{array}{c}\text { Có mức độ } \\
\text { tự chú cao }\end{array}$ & $\begin{array}{c}\text { Hoàn toàn } \\
\text { được tự } \\
\text { chủ }\end{array}$ \\
\hline trong công tác tuyển dụng & 1 & 2 & 3 & 4 & 5 \\
\hline $\begin{array}{l}\text { trong phân phối tiền thưởng và thu nhập tăng thêm } \\
\text { theo kết quả công việc }\end{array}$ & 1 & 2 & 3 & 4 & 5 \\
\hline trong công tác bổ nhiệm & 1 & 2 & 3 & 4 & 5 \\
\hline
\end{tabular}




\begin{tabular}{|l|c|c|c|c|c|}
\hline trong công tác bố trí, sắp xếp nhân viên & 1 & 2 & 3 & 4 & 5 \\
\hline $\begin{array}{l}\text { trong chuyển đổi vị trí cho những nhân viên không } \\
\text { đáp ứng yêu cầu công việc }\end{array}$ & 1 & 2 & 3 & 4 & 5 \\
\hline $\begin{array}{l}\text { trong việc cho thôi việc đối với nhân viên không } \\
\text { đáp ứng được yêu cầu công việc }\end{array}$ & 1 & 2 & 3 & 4 & 5 \\
\hline
\end{tabular}

Mức độ nào ông/bà đồng ý hoặc không đồng ý với các phát biểu dưới đây?

\begin{tabular}{|c|c|c|c|c|c|c|c|}
\hline & $\begin{array}{l}\text { Rất } \\
\text { không } \\
\text { đồng ý }\end{array}$ & $\begin{array}{l}\text { Cơ } \\
\text { bản } \\
\text { không } \\
\text { đồng ý }\end{array}$ & $\begin{array}{l}\text { Hoí } \\
\text { không } \\
\text { đồng ý }\end{array}$ & $\begin{array}{c}\text { Không } \\
\text { đồng ý } \\
\text { cũng } \\
\text { không } \\
\text { phản } \\
\text { đối }\end{array}$ & $\begin{array}{l}\text { Hơi } \\
\text { đồng ý }\end{array}$ & $\begin{array}{c}\text { Cơ } \\
\text { bản } \\
\text { đồng ý }\end{array}$ & $\begin{array}{l}\text { Rất } \\
\text { đồng ý }\end{array}$ \\
\hline \multicolumn{8}{|l|}{ Thủ trưởng cơ quan tôi } \\
\hline $\begin{array}{l}\text { thường xuyên có ý tưởng cải tiến căn bản để tăng } \\
\text { cường hiệu quả hoạt động của cơ quan. }\end{array}$ & 1 & 2 & 3 & 4 & 5 & 6 & 7 \\
\hline có niềm đam mê trong công việc & 1 & 2 & 3 & 4 & 5 & 6 & 7 \\
\hline có tầm nhìn về tương lai của cơ quan tôi & 1 & 2 & 3 & 4 & 5 & 6 & 7 \\
\hline $\begin{array}{l}\text { tạo sức ép để chúng tôi làm việc sáng tạo và hiệu } \\
\text { quả hơn }\end{array}$ & 1 & 2 & 3 & 4 & 5 & 6 & 7 \\
\hline $\begin{array}{l}\text { sẵn sàng và dám chấp nhận rủi ro trong các quyết } \\
\text { định của mình }\end{array}$ & 1 & 2 & 3 & 4 & 5 & 6 & 7 \\
\hline $\begin{array}{l}\text { kiên trì, nhẫn lại trong việc theo đuổi các giải pháp } \\
\text { để cải tiến hiệu quả hoạt động của cơ quan }\end{array}$ & 1 & 2 & 3 & 4 & 5 & 6 & 7 \\
\hline $\begin{array}{l}\text { thực sự quan tâm làm sao để cơ quan tôi có thể làm } \\
\text { tốt nhất để phục vụ lợi ích của nhân dân và của đất } \\
\text { nước }\end{array}$ & 1 & 2 & 3 & 4 & 5 & 6 & 7 \\
\hline $\begin{array}{l}\text { thực sự quan tâm đến việc làm sao để cơ quan tôi } \\
\text { có thể hoạt động một cách hiệu quả nhất }\end{array}$ & 1 & 2 & 3 & 4 & 5 & 6 & 7 \\
\hline $\begin{array}{l}\text { thực sự quan tâm đến hoạt động phòng chống tham } \\
\text { nhũng của cơ quan }\end{array}$ & 1 & 2 & 3 & 4 & 5 & 6 & 7 \\
\hline
\end{tabular}

Mức độ nào ông/bà đồng ý hoặc không đồng ý với phát biểu dưới đây về năng lực chuyên môn của bộ phận tổ chức cán bộ ở cơ quan mình? 


\begin{tabular}{|c|c|c|c|c|c|c|c|}
\hline & $\begin{array}{c}\text { Rất } \\
\text { không } \\
\text { đồng ý }\end{array}$ & $\begin{array}{l}\text { Cơ } \\
\text { bản } \\
\text { không } \\
\text { đồng ý }\end{array}$ & $\begin{array}{l}\text { Hoì } \\
\text { không } \\
\text { đồng ý }\end{array}$ & $\begin{array}{c}\text { Không } \\
\text { đồng ý } \\
\text { cũng } \\
\text { không } \\
\text { phản } \\
\text { đối }\end{array}$ & $\begin{array}{l}\text { Hơi } \\
\text { đồng ý }\end{array}$ & $\begin{array}{c}\text { Cơ } \\
\text { bản } \\
\text { đồng ý }\end{array}$ & $\begin{array}{l}\text { Rất } \\
\text { đồng ý }\end{array}$ \\
\hline $\begin{array}{l}\text { Bộ phận tổ chức cán bộ ở cơ quan tôi có đủ chuyên } \\
\text { môn để xây dựng và triển khai hệ thống đánh giá } \\
\text { dựa trên mục tiêu một cách hiệu quả }\end{array}$ & 1 & 2 & 3 & 4 & 5 & 6 & 7 \\
\hline $\begin{array}{l}\text { Bộ phận tổ chức cán bộ ở cơ quan tôi có thể xây } \\
\text { dựng các tiêu chí đánh giá kêt quả thực hiện công } \\
\text { việc một cách rõ ràng và cụ thể }\end{array}$ & 1 & 2 & 3 & 4 & 5 & 6 & 7 \\
\hline $\begin{array}{l}\text { Bộ phận tổ chức cán bộ ở cơ quan tôi có đủ năng } \\
\text { lực đế thiết kế một hệ thống đánh giá phân biệt kêtt } \\
\text { quả làm việc của từng người một cách công bằng } \\
\text { và khách quan }\end{array}$ & 1 & 2 & 3 & 4 & 5 & 6 & 7 \\
\hline $\begin{array}{l}\text { Bộ phận tổ chức cán bộ ở cơ quan tôi có đủ năng } \\
\text { lực đế xây dựng một hệ thông phân phối tiền lương } \\
\text { dựa trên kết quả công việc một cách hiệu quả }\end{array}$ & 1 & 2 & 3 & 4 & 5 & 6 & 7 \\
\hline $\begin{array}{l}\text { Bộ phận tổ chức cán bộ của chúng tôi có thề tư vấn, } \\
\text { hố trợ các phòng, ban khác các vấn đề về quản lý } \\
\text { công chức, viên chức một cách hiệu quả }\end{array}$ & 1 & 2 & 3 & 4 & 5 & 6 & 7 \\
\hline $\begin{array}{l}\text { Xét một cách tổng thể, bộ phận tổ chức cán bộ của } \\
\text { chúng tôi có chuyên môn sâu về công tác quản lý } \\
\text { và sứ dụng công chức, viên chức }\end{array}$ & 1 & 2 & 3 & 4 & 5 & 6 & 7 \\
\hline
\end{tabular}

Mức độ nào ông/bà đồng ý hoặc không đồng ý với các phát biểu dưới đây về thực tế ở cơ quan mình?

\begin{tabular}{|c|c|c|c|c|c|c|c|}
\hline & $\begin{array}{l}\text { Rất } \\
\text { không } \\
\text { đồng ý }\end{array}$ & $\begin{array}{c}\text { Co } \\
\text { bản } \\
\text { không } \\
\text { dồng ý }\end{array}$ & $\begin{array}{c}\text { Hoi } \\
\text { không } \\
\text { đồng ý }\end{array}$ & $\begin{array}{c}\text { Không } \\
\text { đồng ý } \\
\text { cũng } \\
\text { không } \\
\text { phản } \\
\text { đối }\end{array}$ & $\begin{array}{l}\text { Hơi } \\
\text { đồng ý }\end{array}$ & $\begin{array}{c}\text { Cơ } \\
\text { bản } \\
\text { đồng ý }\end{array}$ & $\begin{array}{l}\text { Rất } \\
\text { đồng ý }\end{array}$ \\
\hline \multicolumn{8}{|l|}{ Ở nơi tôi làm việc, } \\
\hline một số người vào cơ quan là nhờ các mối quan hệ & 1 & 2 & 3 & 4 & 5 & 6 & 7 \\
\hline $\begin{array}{l}\text { những người có quan hệ tốt với cấp trên sẽ được ưu } \\
\text { tiên trong công việc hơn so với người khác }\end{array}$ & 1 & 2 & 3 & 4 & 5 & 6 & 7 \\
\hline $\begin{array}{l}\text { một người sẽ có nhiều cơ hội được bổ nhiệm hơn } \\
\text { nếu có quan hệ tốt với cấp trên }\end{array}$ & 1 & 2 & 3 & 4 & 5 & 6 & 7 \\
\hline
\end{tabular}




\begin{tabular}{|l|l|l|l|l|l|l|l|}
\hline $\begin{array}{l}\text { cấp trên sẽ khó đánh giá một cách khách quan và } \\
\text { chính xác bởi vì họ muốn duy trì quan hệ tốt với } \\
\text { nhân viên }\end{array}$ & 1 & 2 & 3 & 4 & 5 & 6 & 7 \\
\hline $\begin{array}{l}\text { việc cử đi tham gia các khóa đào tạo, bồi dưỡng có } \\
\text { số lượng hạn chế thường bị ảnh hưởng bởi yếu tố } \\
\text { quan hệ }\end{array}$ & 1 & 2 & 3 & 4 & 5 & 6 & 7 \\
\hline
\end{tabular}

Mức độ nào ông/bà đồng ý hoặc không đồng ý với các phát biểu dưới đây về thực tế ở cơ quan mình?

\begin{tabular}{|c|c|c|c|c|c|c|c|}
\hline & $\begin{array}{l}\text { Rất } \\
\text { không } \\
\text { đồng ý }\end{array}$ & $\begin{array}{l}\text { Cơ } \\
\text { bản } \\
\text { không } \\
\text { đồng ý }\end{array}$ & $\begin{array}{l}\text { Hơi } \\
\text { không } \\
\text { đồng ý }\end{array}$ & $\begin{array}{c}\text { Không } \\
\text { đồng ý } \\
\text { cũng } \\
\text { không } \\
\text { phản } \\
\text { đối }\end{array}$ & $\begin{array}{l}\text { Hơi } \\
\text { đồng ý }\end{array}$ & $\begin{array}{c}\text { Cơ } \\
\text { bản } \\
\text { đồng ý }\end{array}$ & $\begin{array}{l}\text { Rất } \\
\text { đồng ý }\end{array}$ \\
\hline \multicolumn{8}{|l|}{ Ở nơi tôi làm việc, } \\
\hline $\begin{array}{l}\text { cán bộ quản lý thường ngại va chạm với những } \\
\text { người có quan hệ họ hàng với lãnh đạo cấp trên }\end{array}$ & 1 & 2 & 3 & 4 & 5 & 6 & 7 \\
\hline $\begin{array}{l}\text { quá trình bổ nhiệm bị ảnh hưởng bởi yếu tố "con } \\
\text { ông cháu cha" }\end{array}$ & 1 & 2 & 3 & 4 & 5 & 6 & 7 \\
\hline $\begin{array}{l}\text { Việc bổ nhiệm người nhà của những vị lãnh đạo } \\
\text { làm ảnh hương tiêu cực đến động cơ làm việc của } \\
\text { nhân viên }\end{array}$ & 1 & 2 & 3 & 4 & 5 & 6 & 7 \\
\hline $\begin{array}{l}\text { người nhà của lãnh đạo thường được bố trí vào vị } \\
\text { trí công việc tốt cho dù năng lực không bằng người } \\
\text { khác }\end{array}$ & 1 & 2 & 3 & 4 & 5 & 6 & 7 \\
\hline $\begin{array}{l}\text { khả năng, kiến thức và kỹ năng không quan trọng } \\
\text { bằng quan hệ "con ông cháu cha" khi bổ nhiệm } \\
\text { nhân sự }\end{array}$ & 1 & 2 & 3 & 4 & 5 & 6 & 7 \\
\hline
\end{tabular}

Mức độ nào ông/bà đồng ý hoặc không đồng ý với phát biểu dưới đây về các hoạt động liên quan đến triển khai hệ thống đánh giá hiện tại?

\begin{tabular}{|c|c|c|c|c|c|c|c|}
\hline & $\begin{array}{l}\text { Rất } \\
\text { không } \\
\text { đồng ý }\end{array}$ & $\begin{array}{l}\text { Cơ } \\
\text { bản } \\
\text { không } \\
\text { đồng ý }\end{array}$ & $\begin{array}{l}\text { Hơi } \\
\text { không } \\
\text { đồng ý }\end{array}$ & $\begin{array}{c}\text { Không } \\
\text { đồng ý } \\
\text { cũng } \\
\text { không } \\
\text { phản } \\
\text { đối }\end{array}$ & $\begin{array}{c}\text { Hơi } \\
\text { đồng ý }\end{array}$ & $\begin{array}{l}\text { Cơ } \\
\text { bản } \\
\text { đồng ý }\end{array}$ & $\begin{array}{c}\text { Rất } \\
\text { đồng ý }\end{array}$ \\
\hline $\begin{array}{l}\text { Cơ quan tôi đã tuyên truyền, giải thích rõ sự cần } \\
\text { thiết của việc triển khai áp dụng hệ thống đánh giá } \\
\text { hiện tại }\end{array}$ & 1 & 2 & 3 & 4 & 5 & 6 & 7 \\
\hline
\end{tabular}




\begin{tabular}{|l|c|c|c|c|c|c|c|}
\hline $\begin{array}{l}\text { Lãnh đạo của chúng tôi động viên mọi người áp } \\
\text { dụng hệ thống đánh giá hiện hành }\end{array}$ & 1 & 2 & 3 & 4 & 5 & 6 & 7 \\
\hline $\begin{array}{l}\text { Chúng tôi được thông báo đầy đủ về tình hình và } \\
\text { kết quả đạt được của việc áp dụng hệ thống đánh } \\
\text { giá hiện tại }\end{array}$ & 1 & 2 & 3 & 4 & 5 & 6 & 7 \\
\hline $\begin{array}{l}\text { Trao đồi hai chiều giữa lãnh đạo và nhân viên về } \\
\text { xây dựng và thực hiện công tác đánh giá là cởi mở } \\
\text { và dân chủ }\end{array}$ & 1 & 2 & 3 & 4 & 5 & 6 & 7 \\
\hline $\begin{array}{l}\text { Các quy định và quy trình liên quan đến hệ thống } \\
\text { đánh giá hiện tại đã được thông báo và giải thích rõ } \\
\text { ràng }\end{array}$ & 1 & 2 & 3 & 4 & 5 & 6 & 7 \\
\hline $\begin{array}{l}\text { Chúng tôi đã được đào tạo, tập huấn đầy đủ về công } \\
\text { tác đánh giá }\end{array}$ & 1 & 2 & 3 & 4 & 5 & 6 & 7 \\
\hline
\end{tabular}

Mức độ nào ông/bà đồng ý hoặc không đồng ý với các phát biểu dưới đây?

\begin{tabular}{|c|c|c|c|c|c|c|c|}
\hline & $\begin{array}{l}\text { Rất } \\
\text { không } \\
\text { đồng ý }\end{array}$ & $\begin{array}{c}\text { Cơ } \\
\text { bản } \\
\text { không } \\
\text { đồng ý }\end{array}$ & $\begin{array}{l}\text { Hoì } \\
\text { không } \\
\text { đồng ý }\end{array}$ & $\begin{array}{c}\text { Không } \\
\text { đồng ý } \\
\text { cũng } \\
\text { không } \\
\text { phản } \\
\text { đối }\end{array}$ & $\begin{array}{l}\text { Hoì } \\
\text { đồng ý }\end{array}$ & $\begin{array}{c}\text { Cơ } \\
\text { bản } \\
\text { đồng ý }\end{array}$ & $\begin{array}{l}\text { Rất } \\
\text { đồng ý }\end{array}$ \\
\hline $\begin{array}{l}\text { Tôi luôn dành hết nỗ lực để thực hiện công việc của } \\
\text { tôi bất kể công việc khó khăn đến đâu }\end{array}$ & 1 & 2 & 3 & 4 & 5 & 6 & 7 \\
\hline $\begin{array}{l}\text { Tôi sẵn sàng bắt đầu ngày làm việc sớm hơn và trở } \\
\text { về muộn hơn để hoàn thành nhiệm vụ }\end{array}$ & 1 & 2 & 3 & 4 & 5 & 6 & 7 \\
\hline $\begin{array}{l}\text { Tôi sẵn sàng gánh vác thêm nhiệm vụ cho dù nó } \\
\text { không thuộc trách nhiệm của tôi }\end{array}$ & 1 & 2 & 3 & 4 & 5 & 6 & 7 \\
\hline $\begin{array}{l}\text { Tôi luôn ưu tiên hoàn thành các công việc đúng thời } \\
\text { hạn }\end{array}$ & 1 & 2 & 3 & 4 & 5 & 6 & 7 \\
\hline $\begin{array}{l}\text { Tôi có hứng thú để dành tối đa nỗ lực và công sức } \\
\text { của mình cho công việc }\end{array}$ & 1 & 2 & 3 & 4 & 5 & 6 & 7 \\
\hline $\begin{array}{l}\text { Tôi có cảm giác mình là một phần quan trọng trong } \\
\text { tồ chức tôi đang công tác }\end{array}$ & 1 & 2 & 3 & 4 & 5 & 6 & 7 \\
\hline $\begin{array}{l}\text { Tôi cảm thấy có sự gắn bó tình cảm với tổ chức tôi } \\
\text { đang công tác }\end{array}$ & 1 & 2 & 3 & 4 & 5 & 6 & 7 \\
\hline $\begin{array}{l}\text { Được làm công việc hiện tại có ý nghĩa rất quan } \\
\text { trọng đối với tôi }\end{array}$ & 1 & 2 & 3 & 4 & 5 & 6 & 7 \\
\hline $\begin{array}{l}\text { Tôi cảm thấy hạnh phúc để làm việc ở cơ quan tôi } \\
\text { cho đến khi nghỉ hưu }\end{array}$ & 1 & 2 & 3 & 4 & 5 & 6 & 7 \\
\hline $\begin{array}{l}\text { Tôi cảm thấy phải có trách nhiệm đối với các vấn } \\
\text { đề mà cơ quan tôi đang gặp phải }\end{array}$ & 1 & 2 & 3 & 4 & 5 & 6 & 7 \\
\hline $\begin{array}{l}\text { Xét một cách tổng thể tôi cảm thấy hài lòng với } \\
\text { công việc hiện tại của tôi. }\end{array}$ & 1 & 2 & 3 & 4 & 5 & 6 & 7 \\
\hline
\end{tabular}




\section{Ông/ bà đồng ý hoặc không đồng ý ở mức độ nào với các phát biểu dưới đây về việc hoàn thành mục tiêu, nhiệm vụ của cơ quan mình?}

\begin{tabular}{|l|c|c|c|c|c|c|c|}
\hline & $\begin{array}{c}\text { Rất } \\
\text { không } \\
\text { đồng ý }\end{array}$ & $\begin{array}{c}\text { Cỏ } \\
\text { bản } \\
\text { không } \\
\text { đồng ý }\end{array}$ & $\begin{array}{c}\text { Hơi } \\
\text { không } \\
\text { đồng ý }\end{array}$ & $\begin{array}{c}\text { Không } \\
\text { đồng ý } \\
\text { cũng } \\
\text { không } \\
\text { phản } \\
\text { đối }\end{array}$ & $\begin{array}{c}\text { Hờ } \\
\text { đồng ý }\end{array}$ & $\begin{array}{c}\text { Cón } \\
\text { bản } \\
\text { đồng ý }\end{array}$ & $\begin{array}{c}\text { Rầt } \\
\text { đồng ý }\end{array}$ \\
\hline $\begin{array}{l}\text { Cơ quan tôi hiện tại đang phát huy tối đa hiệu quả } \\
\text { hoạt động của nó }\end{array}$ & 1 & 2 & 3 & 4 & 5 & 6 & 7 \\
\hline $\begin{array}{l}\text { Mọi người cùng cấp với tôi hài lòng với kết quả } \\
\text { hoạt động của cơ quan tôi }\end{array}$ & 1 & 2 & 3 & 4 & 5 & 6 & 7 \\
\hline $\begin{array}{l}\text { Cơ quan tôi đã và đang làm tốt nhiệm vụ trong việc } \\
\text { đáp ứng yêu cầu của lãnh đạo cấp trên, các cơ quan } \\
\text { liên quan và khách hàng (nếu có) }\end{array}$ & 1 & 2 & 3 & 4 & 5 & 6 & 7 \\
\hline $\begin{array}{l}\text { Cơ quan tôi tạo điều kiện và khuyến khích tôi làm } \\
\text { việc tốt nhất trong khả năng có thể }\end{array}$ & 1 & 2 & 3 & 4 & 5 & 6 & 7 \\
\hline $\begin{array}{l}\text { Lãnh đạo cấp trên, các cơ quan liên quan và khách } \\
\text { hàng (nếu có) đánh giá cao kết quả hoạt động của } \\
\text { cơ quan tôi }\end{array}$ & 1 & 2 & 3 & 4 & 5 & 6 & 7 \\
\hline
\end{tabular}

Theo ông bà cái gì là yếu tố quan trọng nhất ảnh hưởng đến hiệu quả của hệ thống đánh giá kết quả thực hiện nhiệm vụ của công chức, viên chức?

Theo ông/bà những gì có thể được làm để cải thiện kết quả thực hiện nhiệm vụ của đội ngũ công chức, viên chức nói chung trong thời gian tới?

.Cám ơn ông/bà đã dành thời gian để hoàn thành phiếu khảo sát này. Những đóng góp của ông bà sẽ rất có ích để giúp chúng tôi hoàn thành tốt dự án nghiên cứu của mình.

Xin vui lòng bỏ phiếu khảo sát vào phòng bì gửi kèm có sẵn địa chỉ và gửi lại giúp chúng tôi trước ngày 30 tháng 11 năm 2016./. 
Appendix C: Profile of interviewees

\begin{tabular}{|c|c|c|c|c|}
\hline Interviewee & Position & $\begin{array}{l}\text { Organisational } \\
\text { category }\end{array}$ & $\begin{array}{l}\text { Organisational } \\
\text { level }\end{array}$ & Gender \\
\hline Interviewee 1 & Senior leader & Administration & Provincial & Male \\
\hline Interviewee 2 & Senior leader & Administration & Provincial & Male \\
\hline Interviewee 3 & HR manager & Administration & Provincial & Male \\
\hline Interviewee 4 & HR manager & Administration & Provincial & Female \\
\hline Interviewee5 & HR manager & Administration & Provincial & Female \\
\hline Interviewee6 & HR manager & Administration & Provincial & Male \\
\hline Interviewee7 & HR manager & Hospital & Provincial & Female \\
\hline Interviewee8 & Senior manager & Administration & Provincial & Male \\
\hline Interviewee9 & Staff & Administration & Provincial & Male \\
\hline Interviewee10 & HR manager & Administration & Provincial & Male \\
\hline Interviewee11 & HR manager & Administration & District & Male \\
\hline Interviewee12 & HR staff & Hospital & Provincial & Female \\
\hline Interviewee13 & Staff & University & Under Ministry & Male \\
\hline Interviewee14 & HR manager & University & Under Ministry & Female \\
\hline Interviewee15 & HR staff & Research institute & Under Ministry & Female \\
\hline Interviewee16 & Senior manager & Research institute & Under Ministry & Male \\
\hline Interviewee17 & Senior manager & Administration & Under Ministry & Male \\
\hline Interviewee18 & Staff & Research institute & Under Ministry & Female \\
\hline Interviewee19 & Senior manager & Public service & Under Ministry & Male \\
\hline Interviewee20 & Senior manager & Public service & Under Ministry & Male \\
\hline Interviewee21 & HR staff & Public service & Under Ministry & Female \\
\hline Interviewee 22 & Senior manager & Newspaper & Under Ministry & Male \\
\hline Interviewee23 & Staff & Research institute & Under Ministry & Female \\
\hline Interviewee24 & Staff & University & Under Ministry & Male \\
\hline Interviewee25 & Senior manager & University & Under Ministry & Male \\
\hline Interviewee26 & Senior manager & Administration & Under Ministry & Male \\
\hline Interviewee27 & HR manager & Administration & Under Ministry & Male \\
\hline Interviewee28 & Staff & Administration & Under Ministry & Male \\
\hline Interviewee29 & Staff & Administration & Under Ministry & Male \\
\hline Interviewee30 & Staff & Training School & Under Ministry & Male \\
\hline
\end{tabular}




\section{MANAGING EMPLOYEE PERFORMANCE IN TRANSITION ECONOMIES: A STUDY OF VIETNAM PUBLIC ORGANISATIONS}

\section{CONSENT TO INTERVIEW}

This consent form will be held for 5 years.

I have read the Information Sheet and the project has been explained to me. My questions have been answered to my satisfaction. I understand that I can ask further questions at any time.

I agree to take part in a (video/audio) recorded interview.

I understand that:

I may withdraw from this study up to 4 weeks after the interview and any information that I have provided will be returned to me or destroyed.

The information I have provided will be destroyed 03 years after the research is finished.

Any information I provide will be kept confidential to the researcher and the supervisor. I understand that the results will be used for a $P h D$ report and a summary of the results may be used in academic reports and/or presented at conferences.

My name will not be used in reports, nor will any information that would identify me.

[OR]I consent to information or opinions which I have given being attributed to me in any reports on this research

I would like a summary of my interview $\quad$ Yes I would like to receive a summary of the final report and have added my Yes email address below.

Participant signature:

Participant name:

Date:

Contact address 


\section{Appendix E: Semi-structured interview questions for non-managerial employees}

1. Can you describe employee PM in your organisation?

○ What are key objectives of the system?

- What PM practices have been implemented?

○ Do you think that the system have achieved its goals?

- If so, why? Alternatively, if not, why?

2. Which criteria are being used to appraise employees in your organisation?

○ Do you think that these criteria enable managers to rate employee performance accurately, fairly and objectively?

3. In your organisation, is there linkage between performance results and rewards such as monetary bonus, salary increase and promotion?

If so, why?

○ How are they linked?

○ Does this linkage improve employee performance? If so, why?

4. Do employees have opportunities to participate in the process of employee PM such as developing mandates, developing performance standard, goal setting, appraisal, rating and so on?

- If so, how do they participate into these processes?

○ Do your think that employee participation can improve the effectiveness of employee PM in your organisation? If so, why? Alternatively, if not, why?

5. Do you think that the current feedback practice is helpful in improving employee performance?

- If so, why? Alternatively, if not, why?

6. How does your organisation address poor performance?

- Is there anyone who was transferred or terminated because of poor performance?

7. Does your agency have autonomy in the HR practice such as recruitment, rewarding, appraisal and addressing poor performers?

○ Does this autonomy help your organisation innovate employee PM?

- If so, please describe how it take place?

8. How is your agency accountable to political leaders and the upper-agencies? 
○ What has your agency been done to meet these accountabilities?

- How do the accountabilities relate to employee PM?

$\circ$ Is there any relationship between the accountabilities and the support of senior managers for the innovation of performance appraisal system?

9. What role does the personnel unit plays in the renewal or refinement of performance appraisal practices?

- Are you confident that the personnel unit has enough competence to design and support an effective PA system?

- What have they done to help the organisation in improving the current PM system?

10. Do you think that cultural factors (e.g. personal relationships and nepotism) in your organisation affect appraisal practices such as job assignment, feedback, ratings, awarding and addressing poor performance?

- What factors do managers in your organisation often consider when making appraisal decisions, along with competence and contribution?

- How do personal relationships and nepotism affect the implementation of employee PM?

○ Could you give specific examples about the influence of these cultural factors on employee PM?

11. What intervention measures (communication, training...) have been adopted to enhance the effectiveness of the employee PM practice?

○ How are they implemented?

○ Do they help to improve the effectiveness of employee PM?

12. Does the current PM system help to improve your attitude and performance?

- If so, why? Alternatively, if not, why?

13. Do you have any further comments? 


\section{Appendix F: Semi-structured interview questions for HR managers}

1. What are key objectives of the employee PM system in your organisation?

○ What employee PM practices have been implemented?

○ Do you think that the system have achieved the initial goals?

If so, why? Alternatively, if not, why?

2. Which criteria are being used to appraise employees in your organisation?

- Do you think that appraisal criteria in your organisation's appraisal system are measurable and reasonable?

- Are the current criteria good enough to differentiate good performers from poor performers? Why?

3. Does your organisation develop annual goals or plans?

$\circ$ Are employee goals integrated with these strategic goals?

If so, how are they integrated?

4. In your organisation, is there any linkage between performance results and with rewards (monetary bonus, salary increase and promotion?

- If so, how are they linked?

○ Why does your organisation want to link performance results and rewards?

○ To what extent to which these connections affect employee performance in your organisation?

- Alternatively, why does not your organisation link performance results and rewards? In other words, what impedes the linkage between appraisal results and rewards in your agency?

5. Do employees have opportunities to participate in the process of performance appraisal (performance standard development, goal setting, appraisal, rating and so on?

If so, how do they participate into the process of PA?

○ Alternatively, if not, why? What are impeding employee participation in the process of employee PM?

6. Do you think that feedback in the PA system helps employees to improve their performance?

○ If so, why? Alternatively, if not, why?

- According to your opinion, what should be done to make feedback more effective in your organisation? 
7. How does your organisation address poor performance?

- Is there anyone who was transferred or terminated because of poor performance?

8. Does your agency have autonomy in the HR practice such as recruitment, rewarding, appraisal and addressing poor performers?

○ Does this autonomy help your organisation innovate employee PM?

- If so, please describe how it take place?

9. How is your agency accountable to political leaders and the upper-agencies?

- What has your agency been done to meet these accountabilities?

- How do the accountabilities relate to the introduction and the refinement of employee PM practices?

$\circ$ Is there any relationship between the accountabilities and the support of senior managers for the innovation of PM system?

10. What is the role of chief executives in innovating employee PM?

11. Do you think that personal relationships and nepotism in your organisation affect employee PM such as goal setting, feedback, rating and addressing poor performance?

$\circ$ What factors do managers in your organisation often consider when making appraisal decision, along with competence and contribution?

- How do personal relationships and nepotism affect the result of PA?

12. Has your organisation implemented communication and training before and during the implementation process of employee PM?

○ How do they improve the effectiveness of employee PM?

13. Does the current employee PM system help to improve employee attitude and the organisation's performance?

○ If so, why?

- Alternatively, if not, could you please articulate why the current appraisal system cannot improve employee performance?

14. Are there any other contextual factors influencing the innovation and the effectiveness of performance appraisal? If so, please indicate in detail.

15. Do you have any further comments?

Thank you very much for your useful information. 


\section{Appendix G: Semi-structured interview questions for senior managers}

1. What are key objectives of the current employee PM system in your organisation?

○ What PM practices have been implemented in your organisation?

○ Do you think that the system have achieved these goals?

- If so, why? Alternatively, if not, why?

2. Which criteria are being used to appraise employees in your organisation?

- Do you think that appraisal criteria in your organisation's appraisal system are measurable and reasonable?

- Do these criteria enable managers to rate employee performance accurately, fairly and objectively?

3. In your organisation, are employee goals integrated with the strategic goals?

$\circ$ If so, how is this integration implemented?

○ If not, what impedes this integration?

4. In your organisation, is there linkage between performance results and rewards such as monetary bonus, salary increase and promotion?

If so, why? How are they linked?

○ Does this linkage improve employee performance?

- Alternatively, why does not your organisation link performance results and rewards?

5. Do employees have opportunities to participate in the process of PM (developing the PM mandate, developing performance standard, goal setting, appraisal and so on?

If so, how do they participate into the process of PA?

- Do your think that employee participation can improve the effectiveness of performance appraisal in your organisation? If so, why? Alternatively, if not, why?

$\circ$ Alternatively, if not, why? What are impeding employee participation in the process of performance appraisal?

6. Do you think that feedback in the PM system helps employees to improve their performance?

O If so, why? Alternatively, if not, why?

- According to your opinion, what should be done to make feedback more effective in your organisation?

7. How does your organisation address poor performance? 
- Is there anyone who was transferred or terminated because of poor performance?

8. Does your agency have HR and financial autonomy?

- What extent of autonomy does your organisation have?

$\circ$ Is there any innovation concerning employee PM that have been done based on this autonomy?

9. How is your agency accountable to political leaders, the upper-agencies or stakeholders?

○ What has your agency been done to meet these accountabilities?

- How do the accountabilities relate to the innovation of PM practices?

10. What role does the personnel unit play in the renewal or refinement of appraisal practices?

- Are you confident that the personnel unit has enough competence to design and operate an effective PA system?

11. Do you think that personal relationships and nepotism in your organisation affect the PA practice (i.e. job assignment, feedback, rating, rewarding and so on)

- What factors do managers in your organisation often consider when making appraisal decision, along with employee performance and contribution?

- How do personal relationships and nepotism affect the implementation of PA?

12. Has your organisation implemented communication and training before and during the implementation process of employee PM?

○ How are they implemented?

○ How do they improve the effectiveness of employee PM?

13. Does the current PM system help to improve employee performance?

If so, why?

- Alternatively, if not, why? What are main reasons that make the appraisal system to be less effective in improving employee performance?

14. Are there any other contextual factors influencing the development and effectiveness of performance appraisal? If so, please indicate in detail.

15. Do you have any further comments?

Thank you very much for your useful information. 